\title{
Mhos IC
}

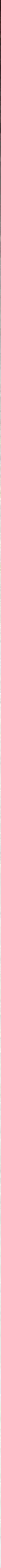




\section{DISCLAIMER}

This report was prepared as an account of work sponsored by an agency of the United States Government. Neither the United States Government nor any agency Thereof, nor any of their employees, makes any warranty, express or implied, or assumes any legal liability or responsibility for the accuracy, completeness, or usefulness of any information, apparatus, product, or process disclosed, or represents that its use would not infringe privately owned rights. Reference herein to any specific commercial product, process, or service by trade name, trademark, manufacturer, or otherwise does not necessarily constitute or imply its endorsement, recommendation, or favoring by the United States Government or any agency thereof. The views and opinions of authors expressed herein do not necessarily state or reflect those of the United States Government or any agency thereof. 


\section{DISCLAIMER}

Portions of this document may be illegible in electronic image products. Images are produced from the best available original document. 
THE

NORMANSKILL HYDROELECTRIC FACILITY

FEASIBILITY

ASSESSMENT

U. S. DEPARTMENT OF ENERGY

CONTRACT EW-78-F-07-1765

\section{Co-sponsors:}

New York State Energy Research and Development Authority City of Watervliet, New York

Contractor: $95 ! 0014$

J. Kenneth Fraser and Associates, P.C. Consulting Engineers

Rensselaer, New York

Principal Investigator: James A. Besha, P.E.

Hydrologic Phase:

Marketing Phase:

Configuration Phase:

Environmental Phase:

Licensing \& Regulatory Phase:

Economic Analysis:

Implementation Plan:
Robert A. Fraser, P.E. Michael E. Gilchrist Joseph Costanza, I.E. J. Daniel Wojick, L.A. James A. Besha, P.E. James A. Besha, P.E. Robert A. Fraser, P.E. 
Chapter

1 Introduction and Overview Figure 1

2 Hydrologic - Segment one Background and Scope 2-1 Introduction $2-2$

Data Base 2. 2-2

Physical Survey 2-7

Streamflow Synthesis 2-8

Watershed Soil Loss 2-9

Capacity Determination 2-10

Yield Analysis 2-12

Discussion of Error 2-12

Future Impacts 2-13

Conclusions 2-16

Appendix A 2-19

Appendix B . $2-24$

Appendix C $2-25$

Appendix D 2-27

Appendix E 2-28

Appendix $F \quad 2-30$

Figures 2-10

3 Hydrologic - Segment Two

Data Base- 3-1

Spillway Capacity and Overflow Capacity 3-7

Flow Duration 3-8

Initial Operational Model 3-9

Refinements to Operational Model 3-12.

Conclusions 3-14

Figures $11-29$

4 Marketing

Electric Power Market

Municipal Power Consumption : $4-4$

Benefit Value Program 4-7

Utility Interface 4-8

Incurred Municipal Benefits and Costs 4-10

Figures $30-30 \mathrm{~A}$

5 Configuration

Existing Facilities

Site Options

Machine Options

Costs

Optimization Methodology

$4-1$

$4-8$
$4-10$

Figures 31-44 
Environment

Reservoir Related

$6-1$

St.ream Related

$6-9$

Recreational

$6-13$

Other Impacts

$6-14$

Cultural and Historical

$6-15$

Pertinent Statutes and Regulations

$6-20$

Statute Applicability

$6-26$

Figures $45-55$

7. Licensing and Regulatory Requirements

Jurisdiction

Review Requirements

Critical. Energy Facility. Program

Environmental and Cultural Regulatory

Requirements

$7-1$

$7-1$

$7-2$

$7-3$

8 Economic

Selected Projects

Determination of Discount Rate and Gradients

8-2

Present Worth Analysis

Benefit Cost Ratio

$8-3$

$8-5$

$8-6$

Cash Flows

Delta Cash Flow

$8-8$

Discounted Cash Flow

8- -9

Subjective Considerations

8-10

Sensititivity Analysis

$8-11$

Criteria for Incremental. Projects

$8-12$

Conclusions

$8-13$

8-16

Figures $56-67$

9 Implementation

PERT

Financing

9-1

$9-2$

Figures 68-69

10 Synopsis and Recommendations

Recommended Project

Economic Criteria

$10-1$

Conclusions

$10-5$

Further Criteria

$10-7$

Figures $70-77$

11. Selected Bibliogrāphy and Referenoes

11-1 


\section{FIGURES}

\section{Chapter 1}

1 Analysis Flow Chart

\section{Chapter 2}

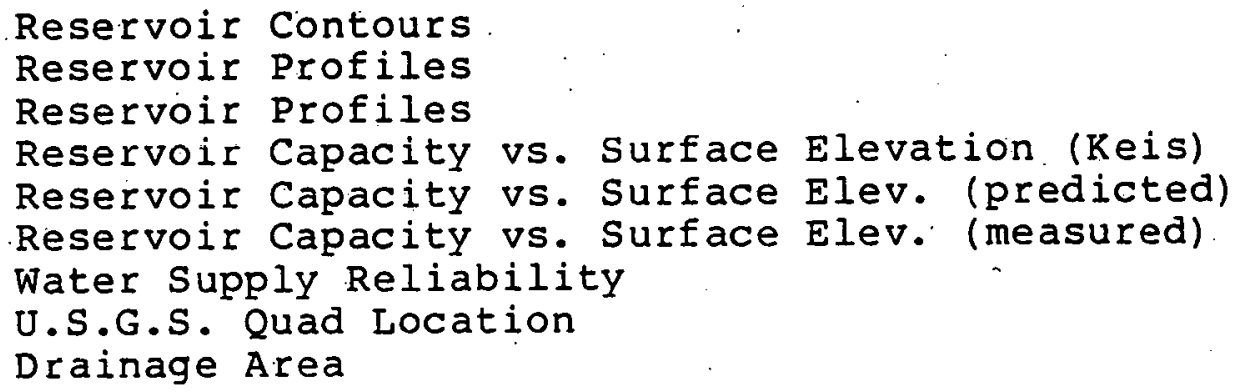

\section{Chapter 3}

11

12

13

14

15

16

Monthly Flow Probability - January

Monthly Flow Probability - February

Monthly Flow Probability - March

Monthly Flow Probability - April

Monthly Flow Probability - May

Monthly Flow Probability - June

Monthly Flow Probability - July

Monthly Flow Probability - August

Monthly Flow Probability - September

Monthly Flow Probability - October

Monthly. Flow Probability - November

Monthly Flow Probability. - December

Monthly Flow Probability

Annual Turbine operation

-Operating Time vs. Turbine Capacity

Annual Turbine operation

-Operating Time vs. Turbine Capacity

Reservoir simulation Output

Power Production

- Power vs. Turbine Capacity

29 Power vs. Capacity

\section{Chapter 4}

30 Municipal Electric Consumption

30A Benefit Value Program Output (BVP) 


\section{Chapter 5}

31 Site Location in SMSA

3IA Site Location (U.S.G.S.)

32 Water Supply system (Keis 1915)

33 Plan and Profile (Keis 1915)

Dam Plan and Profile

Dam-Section

Existing Penstock at Intake - Photo

Normans Kill below Dam - Photo

Existing Turbines and Pumps - Photo

Existing Switchgear - Photo

$41 \mathrm{~A}$ Site Options 1.03 thru 1.03

41B Site Options 2.01 thru 2.12

41C Site Option 3

4ID Site Options 4.01 thru 4.11

$41 \mathrm{E}$ Site Options 5.02 thru 5.13

$41 \mathrm{~F}$ Site Options 6.01 thru 6.13

$41 \mathrm{G}$ Site Options 7.01 thru 7.113

$41 \mathrm{H}$ Site options 8.01 thru 8.13

42 Penstock Unit cost

43 N.M.P.C. Distribution System

44 Optimum Configuration Program (OCP)

\section{Chapter 6}

45

Environmental - Stream Related

Environmental - Land Use and Recreation

Environmental - Vegetation

Environmental - Surface Conditions

Bleeker Map - 1767

Beers Map - 1886

Gould \& Moore Map - 1854

Covered Bridge - (date unknown). - Photo

Spawn Home-North Face - Photo

spawn Home-Entrance Details - Photo

Spawn Barn - Photo

\section{Chapter 8}

Benefit/Cost Ratio

Discounted Cash Flow Internal Rate of Return Cash Flows

Sensitivity - Benefits vs. Discount vs. Period

Sensitivity - B/C Ratio vs. Discount v.s. Period

Data Matrix for Figures 59 and 60

Sensitivity - Net. Benefits vs. Capital Cost vs. Period Sensitivity - B/C Ratio vs. Capital Cost vs. Period Data Matrix for Figures 62 and 63

Sensitivity - Benefits vs. Energy vs. Labor

Sensitivity - B/C Ratio vs. Energy vs. Labor

Data Matrix for Figures 65 and 66 


\section{Chapter 9}

68 Property Map

69 PERT Chart

70 Selected Option No. 8.07

71 Proposed Intake at Dam

$71 \mathrm{~A}$ Proposed Intake Section

72 Plan - Powerhouse

73 Powerhouse and Access

73A Powerhouse and Access

74 Control/Pump Station Modification

75 Electrical Schematic

76 Control Schematic

77 Proposed Project Credits and Disbursements 
The Normanskill Hydroelectric Facility Feasibility Assessment has been a multidisciplinary evaluation of the hydroelectric generating potential at the City of watervliet water supply Reservoir and the associated recreational, environmental, historical, archeological and institutional constraints. In completing this feasibility assessment, many of the analyses have been performed concurrently. As an aid to understanding the completed feasibility assessment, a program flow chart (Figure.1) has been prepared graphically interpreting the various engineering analyses and their relationships.

since much of the analysis has involved modeling of the various systems, a brief description of the generalized modeling process follows. The planning of water resource systems is dependent upon information regarding hydrologic phenomena. The data base available for such phenomena is seldom large enough to provide precise information and as a result are subject to uncertainties. These uncertainties can be reduced by the use of more extensive data bases. The delays in obtaining a more extensive hydrologic data base, however, is in the order of decades. A more feasible course of action is to develop and use mathematical models of certain processes in order to extrapolate information over time and space. The models, themselves, do not predict with certainty the values of specific variables at any one point in time. However, the information generated from such models can be generated quickly and objectively and can provide a quantitative measure of the quality of the generated information. The models used in this feasibility assessment are of two types, stochastic (Markov chain) and simulation type. The core and initial model used in this hydroelectric feasibility assessment is one which uses a stochastic process to generate sequences of hypothetical streamflows such that any streamflows can be regarded as an equally likely realization of any future flow sequence. This: will be explained more fully in the hydrologic phases of the report: The second technique used 
extensively in this analysis is simulation. In this technique, the uncertainties, interactions and variables are stored in the memory of a digital computer. The simulation begins at a specified starting state and following that start, the combined effects of decisions, interactions, etc. cause the system to progress in simulated time. A vast number of calculations are performed in a short period by the computer. A major feature of this technique is time compression.

The various assessment tasks described hereafter are depicted as having been discrete and clearly defined modules which have been interconnected defining a program and giving an end result. Such clarity and definitiveness can never be obtained but is used as a convenience in describing the methodology.

The first major analysis performed was development of the stochastic model which was used to extrapolate the ten-year stream flow data base. The model yielded a hydrologic stream flow simulation over a 500-year trace. Following development of this model, multiple simulations involving the model were run as a means of developing the mass balance characteristics of the City of Watervliet water supply reservoir.

These multiple runs were performed using various combinations of water supply usage, draft assumptions, etc. as a means of defining the response of the reservoir system to water supply, hydroelectric generation and recreational demands.

Concurrently, following a detailed physical investigation of the site and as a result of several brainstorming sessions among all assessment participants, eight selected alternatives or schemes were adopted for further investigation. These eight alternatives were then methodically scrutinized and further defined. For each alternative, several values of pertinent variables were selected yielding further permutations of the basic eight alternatives. Eventually 78 alternatives, each with associated specific capital and operating costs were defined. Concurrent with this effort, data was collected on the City of Watervilet power consumption and costs for the past several years. This data along with pertinent 
assumptions on projected future energy consumption served as input to a computer model that evaluated, interpolated, extrapolated and analyzed the City's power requirements and yielded a "typical year" for energy consumption by the City of Watervliet municipal

facilities.

The data from this energy use program as well as values representing the power production capabilities of the 78 alternatives and the result of numerous hydrologic reservoir system simulations served as input to a benefit value program. This benefit value program determined the economic benefits attributable to the $78^{\prime}$ selected alternatives. The value in each case was a single cost (or savings) allowable to the City through reduced consumption of commercial power.

The results of the benefit value analysis were compared to capital, operating, and maintenance costs and ranked. This analysis was performed in an optimum configuration program which yielded net benefits as well as rankings based upon least cost for each of the 7.8 options. Implicit in this cost analysis were certain assumptions relating to bonding and discount rates, and cost escalation gradients. In order to properly determine the sensitivity of the analysis to these factors, multiple evaluations were made using a range for each of the factors.

Concurrent with the configuration analysis, an environmental, cultural, historical, archaeological and recreational analysis was performed for the reservoir system in general and for several of the most promising alternatives specifically.

Using the data obtained from the environmental analysis as well as the most promising alternatives from the optimum configuration program, an economic analysis has been performed detailing the various costs, benefits and subjective values for two optimum hydroelectric alternatives representing both a rehabilitation type project and a new construction project as well as the baseline or no project alternative.

Implicit in this economic analysis are certain assumptions of bonding and discount rates; cost escalation gradients, life cycle periods and capital cost accuracy. A sensitivity analysis has been 
performed and is included which shows the response and conclusions of the economic analyses resulting from using a range of values for these variables.

Requirements for licensing and regulation have been determined. Data relating to scheduling, financing and planning are included in the implementation section.

The Synopsis and Recommendation section includes details about the recommended project and the pertinent economic criteria. 

HYDROLOGIC - SEGMENT 1

Hydrologic Phase - Segment 1 was funded entirely by the City of Watervliet, New York as fulfillment of a portion of their cost-sharing commitment. This chapter was completed and issued as an autonomous report in September 1978 .

\section{Background and Scope}

This Watervliet Reservoir Yield Analysis and Report was authorized by the City of Watervliet Common Council to ascertain the available water supply yield from the watervliet water Supply Reservoir located at French's Mills in the Town of Guilderland, New York. In addition, the need to predict the remaining life expectancy of the reservoir, as affected by sedimentation and eutrophication, is necessary in order to conduct long-range planning for future water supply requirements for the City of Watervliet, New York. A proposal was submitted on July 26, 1977 delineating three alternative schemes for accomplishing this water supply yield study. Alternative Three was chosen by the City Council as the most cost-effective alternative. Briefly stated, that alternative "would accept as given the volumetric capacity of the reservoir developed in 1912 by the original water supply engineers, Solomon \& Keis. From this given reservoir capacity, the expected sedimentation and bank erosion deductions would be made. The sedimentation and bank erosion quantities would be developed and a physical spot verification of expected sedimentation would be attempted under this alternative... actual and synthetic stream flow and rainfall data would be used to conduct a safe yield analysis for the reservoir to determine the maximum safe water supply yield."

It was further noted, in the proposal, that long-term hydrologic data was thought to be generally unavailable but that as part of the yield study, "an exhaustive record would be made to augment the data base to the fullest possible extent. 


\section{Introduction}

The Watervliet reservoir yield analysis has been completed in a manner outlined under the previous section. For purposes of this report, the component parts of the analysis will be briefly described. Detailed methodologies of the various technical elements of the analysis have been included as appendices in order to provide documentation to facilitate verification of this effort in the future. The modular analytical elements used in the determination of the water supply yield are as follows:
A. Data Base
B. Physical Survey
C. Stream Flow Synthesis
D. Watershed Soil Loss
E. Capacity Determination
F. Yield Analysis
G. Error Analysis
H. Future Impacts

Following discussion of each of these component parts, a section entitled Conclusions has been prepared, summarizing the results of this analysis.

\section{Data Base}

The Normans Kill watershed has been considered as a possible water source since the early Nineteenth Century. Most early studies dealt with the possible use of the Normans kill watershed as a potable water supply for the City of Albany. A report prepared in 1850 by Mr. William J. McAlpine [Ref. 72] dealt, in general, with several possible sources of supply, covering the Normans Kill only briefly. The only flow gauging noted by McAlpine is a single value of $8.218 \mathrm{mgd}$ for the Normans Kill at French's Mills. McAlpine further states that the gauging was not performed by him and "are supposed to be the result of one or two trials only." Furthermore, he states, "It is not believed to be possible to ascertain the minimum flow of a stream, without very careful examinations continued over a considerable period of time"[Ref. 72]. 
The second serious consideration for use of the Normans Kill as a water supply resulted on March 16, 1891 of the submission of a report to the Albany Common Council by a Special Water Commission, appointed and authorized by the state Legislature, which recommended the adoption of a plan for securing additional water supply from the Normans kill. The Common Council noted in the public record that the estimates of flow appeared "unexpectedly large" and after deliberating, decided that the "practicability of the entire project depends to no small extent upon the accuracy of these estimates [Ref. 64]. The Council then recommended that the special Commission undertake a further study including measurements of the possible stream flow. It should be noted that the initial measurements made for the Commission by Mr. J. Herbert Shedd, were thought to be highly suspect since the R. D. Wood \& Company, who employed M.r. shedd, was thought to be desirous of securing the contract for constructing the proposed impoundment. The revised estimates prepared by this special commission showed that the shedd estimates were, in fact, 5508 higher than that found during subsequent testing. To confuse things more, the Common council noted that through a "curious oversight, the safe yield computation neglected to take into account the 51 million gallons per day that would have had to have been released from the impoundment to furnish the existing mills located at a point downstream" [Ref. 68]. Discussion of the Normans Kill as a source of supply at that time also involved serious consideration regarding the suitability of the water due to its bacteriological quality. This concern, in 1891, was for the most part fueled by the rapidly changing composition of the watershed in the late $1800^{\prime} \mathrm{s}$ from a primarily forested area to agricultural usage. It should be noted that the entire data base that was used for discussion at that time involved so-called "average" daily flows which were actually based on single daily measurements over a time period thought to be no longer than a year. The methodology by which this sparse data was transformed into a "safe yield" was extremely simplistic, being based on intuition and judgment rather than on any mathematical analysis. 
The Albany Special Water Commission further investigated Lake George, Warner's Lake, Thompson's Lake, Kinderhook Creek, and Schoharie Creek as potential sources of water supply. In addition, several underground sources were investigated. The Albany Common Council eventually selected the Hudson River as a source of supply. The designer and engineers of the existing Normans Kill impoundment and dam, Messrs. Solomon, Norcross \& Keis (hereinafter referred to as "Keis" attempted to determine the available yield by approaching the problem as one of cause and effect. Basically, Keis' safe yield was developed on the basis of a rainfall-runoff ratio. Keis' method assumed that certain portions of the monthly rainfall would run off and be displayed as stream flow. He used percentages ranging from $4 \%$ in July and August to $85 \%$ in March for the runoff ratio. Keis's average rainfall ratio is 39.98 which he compared favorably to the $48 \%$ ratio determined for the Croton (New York City) watershed and a 568 ratio determined for the Philadelphia, Pennsylvania watershed. From examination of all known data, it cannot be determined upon what basis Keis assumed the relative monthly proportions. It should also be noted that this method, while innovative in the early part of this century has been shown to be of little value since the relationship between rainfall and runoff is considerably more complex than a simple ratio. The mechanism of relating rainfall to runoff involves numerous parameters such as watershed area, soil types, soil porosities, geological composition, distribution of storm events, etc. At present, the state of the art is such that highly accurate rainfall runoff prediction models are valid only for extremely small watersheds under tightly constrained circumstances. In any event, Keis never totally addressed the problem of determining a probable safe yield. The amount of water supply required for the City of Watervliet at that time was $4.0 \mathrm{mgd}$. The facilities were designed with this amount in mind, but through what may have been an oversight, it appears that the designer neglected to account for the large additional water supply requirement for the hydraulaic pumping of the water. From various letters, charts, and graphs on file with 
the New York State Resources Commission, it is evident that the State Water Resources Commission was concerned with this although the final decision did not allude to it. As a result of this computational oversight and due to an apparent lack of adequate turbine operating rules for reservoir operation, it was reported in June of 1918 in a report to the New York State Health Department that during the early months of 1918, the Watervliet reservoir's supply was exhausted. The deficit for several months was supplied from the Hudson River. Based upon charts and computations made at that time, it is surmised that the supply was exhausted due to continued operation of the hydraulaic turbines throughout the 1917 year even though rainfall for that year was substantially below normal and the relative volume of water required for operation of the turbines had predictably increased in magnitude as water surface elevation in the reservoir dropped. It is calculated that water use by the city of watervliet for potable supply and for hydraulic pumping during the latter months of 1917 exceeded 40 million gallons per day.

In 1925, a report was prepared by Nicholas Hill for the City of Albany in regard to Albany's future water supply requirements. One option discussed in the Hill report was construction of a dam on the Normans Kill downstream from the existing Watervliet impoundment. Stream flow data upon which Hill developed his calculations was limited to the data gathered for the 1891 Shedd Report. Hill projected estimated minimum flows using comparative hydrology for consecutive periods of one month to twenty-four months duration. The estimated flows were then compared to the actual 1891 flows. The estimated flows were lower than actual flows although Hill indicated the inclusiveness of the comparison due to the meager 1891 data.

Tin 1964, a study prepared by Parsons, Brinkerhoff, Quade \& Douglas (hereinafter referred to collectively as "Parsons") [Ref. 75] for the City of watervliet dealt briefly with the probable safe yield from the impoundment. Parsons' calculations are based upon an observation in the year 1963, during the drought period of the early 
1960 's, that a continuous withdrawal of $4.3 \mathrm{mgd}$ from the reservoir was maintained without lowering the reservoir water surface elevation. Parsons therefore then conservatively estimated minimum stream flow to be $3.3 \mathrm{mgd}$. Parsons correlated this assumed minimum stream flow to "firm yield" by simply relating the amount of storage in the reservoir based upon an assumed number of days with minimum inflow. Parsons' analysis was pedicated upon drought (no rainfall) periods of 90 and 120 days. Based upon these rather simplistic assumptions, a firm yield assuming 90 days of minimum inflows was calculated to be $21 \mathrm{mgd}$, whereas for 120 days, the firm yield would be $16.6 \mathrm{mgd}$. Parsons also investigated the determination of safe yield by the method of comparative stream hydrology. Comparing the curves of yield per square mile versus available storage for three other watersheds in the region, Parsons projected a safe yield of $0.18 \mathrm{mgd}$ per square mile of watershed for the Normans Kill. The report thereafter concluded that $" 20 \mathrm{mgd}$ is a reasonable estimate of the firm yield of the Watervliet Reservoir." It should be noted that the scope of the 1964 water supply report was limited and detailed investigation of the firm yield was not mandated.

Prior discussions of historical data base serve to illustrate the lack of accurate long-term data and the ill-defined methodologies by which previous decisions on the Normans Rill watershed were made. Current state of the art techniques utilizing sophisticated mathematical modeling still require an accurate data base, although of shorter duration. The data base used in this analysis is derived from five sources and is thought to have excellent reliability and accuracy.

The first data source was the record of stream gaugings on the Normans Kill and Hunger Rill performed by the U.S. Geological Survey. These stream gauging stations are permanent installations constructed in 1967 which continuously record flows in each stream on a year-round basis. The gauging stations are extremely well designed and accurate. Data obtained from this source included daily stream flow averages for the period from October 1967 through September 1977. 
The second data resource was the watervliet Pumping Station operational log prepared [Ref. 96] from 1964 to the present by the current pumping station superintendent, Mr. Charles Luckey. Prior to Mr. Luckey's appointment, daily operational logs were not kept. Mr. Luckey's logs provided us with extremely detailed and accurate information relating to reservoir water surface elevations and mode of operation. Through analysis of Mr. Luckey's daily logs in relation to the U.S.G.S. daily stream flow data, a composite stream flow input into the watervliet reservoir was determined.

The third data resource was detailed soill loss analysis performed by the U.S. Soil Conservation Service as part of a statewide conservation needs inventory [Ref. 30]. This soil loss was performed on a watershed basis for the entire state as a means of determining the severity and extent of soil loss through erosion in the state. Both raw and final data from the soil conservation Service offices in Albany and Schenectady Counties were used in our analysis to determine calculated soil losses and expected soil loss input to the reservoir.

The fourth data resource was the Land Use Natural Resources (LUNR) inventory [Ref. 18] prepared by the State of New York. This land use data allowed the definition of sub-watershed areas that were excluded from the sCs soil loss inventory.

The fifth data base resource was extensive rainfall data available from 1820 to the present furnished by the National Oceanic and Atmospheric Administration from the recording stations in the Albany vicinity.

\section{Physical Survey}

The first stage of the physical survey involved comparison of the boundaries depicted on the original reservoir land taking map prepared in 1912 by Solomon, Norcross \& Keis with aerial photography taken in 1977 for the Albany county tax base mapping project. The general reservoir outline was found to correlate fairly well with the Solomon, Norcross \& Keis map except where bank erosion had taken place in certain areas. Generally, these areas were defined by steep clay banks of Hudson silt composition, as verified by on-site investigation. 
A second facet of the physical survey involved the extraction and analysis of core samples from the reservoir bottom in ten locations. This sampling was performed by Empire soils Investigations, Inc. An analysis of the samples to determine unit weights and submerged unit weights was undertaken to verify and calibrate the sedimentation load model subsequently developed.

The third facet of the physical survey involved spot soundings performed by a survey crew in five different areas of the reservoir. These areas called "ranges" were transverse survey lines from which cross-sections were plotted. These actual cross-sections were compared to cross-sections plotted from Solomon, Norcross \& Keis' original land taking map and are shown as Figures 3 and 4 . A comparison of these cross-sections on a statistical basis permitted further definition of the actual siltation that has taken place in the reservoir in the 62 years since its construction.

\section{Stream Flow Synthesis}

A calculation of a reservoir safe yield requires a reasonable data base of stream fiow over an extended period of time. As noted previously, no long-term stream flow data exists for this watershed. The methodology used in our analysis was developed in the 1960's at Harvard University and involves what is today known as "operational hydrology." This approach differs from conventional comparative hydrology in that the data used in the analysis is derived from the specific waterhsed in question. This data is further manipulated and augmented by statistical methods to create a reliable and accurate long-term model of stream flow. Comparative hydrology, on the other hand, relates safe yield or parameters in one watershed with those empirically determined through long-term monitoring for another watershed, ignoring the multitude of differences between them. The term "synthetic hydrology" although used frequently in this report and other publications, is not a totally satisfactory designation because it connotes that fictitious stream flow values are created in such a way that they could not occur at the site. This is not an accurate portrayal. The data 
generated is not actually expected. to be observed, but serves as a substitute data base to further derive other mathematical models which are then used to determine the storage-yield relationships. In this analysis, the stream flow synthesis derived its data base from ten years of monthly reservoir stream inflow values determined from available records. Based upon examination and analysis of these values, a statistical relationship was developed that adequately represented this historical record. Using these historical records as a model, additional synthetic records were generated, representing stream flows for a 500-year period. These flows were partially comprised of a deterministic component representing the historical sample and partially of a random component representing the statistical model assumed. The 500-year synthetic record is statistically identical in most respects to the 10-year record from which it was modeled. It should be noted that while the lo-year historical record is not an extremely long period from which to model stream flow, based upon extensive research to date, it is believed to be adequate.

\section{Watershed Soil Loss}

As a means of determining the actual physical capacity reduction of the reservoir by sedimentation, a watershed soil loss model was constructed. This model relies upon the Universal soil Loss Equation developed by the U.S. Department of Agriculture and now in general use. From this model, an expected annual soil loss in the watershed was developed and was further related to the actual amount of soil transported by streams and deposited in the reservoir. This model, while representing the expected soil loss in a specific year (in this case 1974) can be extrapolated backwards and forwards to arrived at a probable sedimentation load presently in the reservoir and an expected sedimentation load in future years. These extrapolations are based upon assumptions concerning continued land use, conservation practices, etc. Data used in the construction of this model was furnished by the United states soil. Conservation service (SCS) offices in Albany and schenectady 
Counties. The bulk of this data was prepared by the scs offices for a state-wide soil loss inventory prepared recently. For purposes of our model, the SCS raw data was spot-verified and disaggregated to exclude portions of the watershed not tributary to the watervliet Reservoir. Disaggregation of excluded areas was performed using the New York State Land Use Natural Resources Inventory.

A total annual soil loss was further disaggregated into monthly. soil loss. Sediment transport mechanisms delivering soil. loss to the reservoir were re-evaluated on a monthly basis, and reservoir trap efficiencies were likewise re-evaluated. Comparison, on a monthly basis, was necessary due to the extreme range of values of monthly stream flows versus reservoir capacity. The extreme range in this case results from the relatively small physical capacity of the watervliet Reservoir in relation to the overall. watershed area. Computation of annual sedimentation of the Watervliet Reservoir was made on the basis of the calculated monthly loadings.

Using empirical submerged soil densities and sediment densities dervied during the physical survey portion of the study, sediment volume was then computed. Distribution of the sediment was further predicted using the Area-Increment procedure to apportion expected sedimentation buildup over discrete elevations of the reservoir bottom.

\section{Capacity Determination}

The problem of determining the present physical capacity of the reservoir was approached in several different ways.

The physical capacity of the reservoir which was determined by Solomon, Norcross \& Keis from the topographic survey which they prepared for the reservoir construction, was examined in an attempt to arrive at a statistical measure of error to be applied to the original capacity. As described in the Physical survey section, aerial photography confirmed that the water surface area of the reservoir as outlined on the original topographic survey map was of acceptable accuracy. It was assumed, therefore, that the accuracy 
of all other topographic contours within the impoundment could be assumed to have acceptable accuracy as well. Since the original topographic survey was based upon a 5 foot contour interval, the elevations depicted on the topographic map statistically could be a half interval or 2-1/2 feet higher or lower than that shown. Independent computations of capacity versus contour elevation were made under this study using the original topographic mapping. similarly, computations were made using the assumptions of a consistent half-interval contour error. This computation yielded a capacity based upon the original topographic mapping, which we have called "Keis Mean", "Keis High", and "Keis Low" in the graphical. representation found in Figure 5. The Keis High capacity is the most liberal estimate of original capacity assuming that all topographic elevations were actually lower than depicted. The Keis Low capacity is a conservative estimte of reservoir capacity based upon the opposite assumption.

The reduction in reservoir capacity which was predicted by the watershed soil loss computation for the reservoir's sixty-two year history, and also for a one-hundred year period, was then applied to the original reservoir capacity curve. The resulting family of capacity curves representing the reservoir's original 1916 assumed capacity, as well as the projected 1978 and 2016 curves, are represented in Figure 6 . The total volume reductions based upon soil loss computations correspond favorably with losses determined empirically for this type of reservoir and watershed. The total reductions are $7.5 \%$ and 128 for a 62 -year and 100-year 1 ife, respectively.

A second method of capacity determination was based upon the physical survey taken over five selected ranges of the reservoir. sediment buildup found over these ranges in relation to bottom contour as shown on Keis' topographic map were proportionally distributed over all sections and elevations of the reservoir in order to derive a revised capacity curve based upon these actual physical soundings. The physical capacity determined by this method corresponds rather closely with that determined by the theoretical soil loss method. These capacities are depicted graphically in Figures 6 and 7 . 


\section{Yield Analysis}

For a determination of "safe" yield, we have adopted the probabilistic approach now favored. This approach relates the safe yield of the reservoir to the relative frequency of deficit to be expected. By using the record of synthetic stream flows, a reservoir storage simulation model was developed. This model used the 500 year synthetic stream flow record and assumed draft rates to compute the amount of cumulative storage deficit or overflow for each month of the 500 year trace. The frequency of storage deficits, or the number of times in the 500 year record that a given storage volume would be inadequate at a given draft rate was determined. The results of the probabilistic yield analysis are presented graphically in Figure 8. The graph shows the relationship between required reservoir capacity, water supply yield, and the reliability that the yield is "safe." For example, a reliability of $99 \%$ for a selected uniform draft rate implies that the corresponding reservoir capacity or storage deficit would be exceeded once in 100 years at that draft rate whereas a $99.8 \%$ reliability implies that the capacity will be exceeded once in 500 years. For most water supply discussions, a 988 or 998 reliability is normally assumed as a conservative factor. This is owing to the fact that during periods of drought, water use could generally be curtailed as a means for stalling system failure.

Recent research [Ref. 28] indicates that the economic dislocation associated with drought is extremely minor even at the 95\% reliability factor. In a study of several Massachusetts communities following the early 1960's drought, economic dislocation resulting from the drought was approximately $\$ 5.00$ annually per capita. It seems prudent, however, for planning purposes to assume a higher reliability factor of say 998 .

\section{Discussion of Error}

In any scientific analysis the analytical results must be tempered with an understanding of the potential errors inherent in the data and methodology employed. 
Potential errors in this hydrologic analysis can be classified as judgmental, observational or analytical.

Judgmental error is based in the judgment of the analyst where subjective considerations must be given to selection of certain coefficients and variables. A good example of judgmental error source is in the selection of the so-called conservation practice factor used in the Universal Soil Loss Equation.

Observational error results when data being gathered by an observer is recorded inaccurately, usually visually, and goes undetected.

Analytical error is associated with inaccuracies inherent in the analysis itself. These errors result from the simplification of algorithms necessary for computations and from imprecise assumptions or equations required to be able to mathematically represent a process.

Mathematical determination of the magnitude of the range of potential error of the second and third type is possible but is beyond the scope of this study. Determination of judgmental errors is also possible using various methods of psychological testing. For purposes of this study, we have attempted to identify all recognizable sources of error and subjectively discuss them as to probability of occurrence.

A tabulation of errors and probability of occurrence is included as Appendix $F$ following this chapter.

\section{Future Impacts}

The major factor relating to future changes in the capacity of the Waterviliet Reservoir is the land use patterns in the Normans Kill watershed. As depicted on Figure 10, the Normans Kill watershed upstream from the Watervliet Reservoir lies largely in the Town of Guilderland and the Town of Rotterdam. A small portion of the watershed lies in the Town of Wright, Schoharie County. The present land use patterns in the watershed are similar to those of the last 62 years. Change has taken place primarily relating to the development of cropland and an ebb: and flow of woodland in the 
watershed over the past three-quarters of the century. From what can be gleaned from historical sources and from recollections of the Soil Conservation service personnel, the total makeup in agricultural land today is not unlike that of 30-40 years ago. It is surmised that erosion conditions today may be somewhat intensified due to the mechanization of agriculture in the watershed. However, the majority of cropland in the watershed is used for dairy farming and thus is not highly cultivated. The loss of woodland to agriculture is thought to be minimal. Approximately 40\% of the watershed area is classified as forest lands. The primary impact of land use changes in the watershed have been the intensification of land uses associated with the urban use category. At present, approximately 28,600 acres of the total watershed area of 139,100 acres are classified as urban land use. While the bulk of this urban land use lies in the eastern portion of the watershed downstream from the Watervilet Reservoir impoundment, the historical trend has been for a slow westward movement of the urbanization (suburbanization) into portions of the watershed tributary to the reservoir. Up to now, most of this urbanization of the watershed has been through road construction and single family and multiple family residences. It is expected, however, that development of large tracts for apartment complexes will occur in the near future. This trend toward urban use affects the rainfall runoff ratio and the sedimentation rate. "It is interesting to note that in this particular watershed due to its large size in relation to the reservoir capacity, the effect of changes in the rainfall runoff patterns due to urbanization will be largely undetectable due to the immense size of the watershed. Sedimentation; however, as a by-product of the increased runoff rates will be noticeable due to the generally silty nature of the soils and unstable bank condition of the major streams.

The second major impact upon the reservoir capacity is the accelerating eutrophication of the reservoir. This natural process in the life cycle of a body of water has as its causative components; sedimentation, increased vegetative growth, and 
increased flow of nutrients into the impoundment. The effects of this eutrophication are two-fold. The first, the gradual deterioration of water supply due to proliferation of algae growth and lessening of oxygen available for natural cleansing and growth of desirous life forms, will not be detailed in this report. It is obvious, however, that this reduction of water quality will cause a significant cost escalation as more advanced treatment methods become necessary to satisfy the stringent requirements of the U.S. Safe Drinking Water Act.

The major effect of eutrophication, relating to this study, is the tendency of the eutrophied reservoir, with a preponderance of vegetation, to significantly increase its rate of sedimentation. Heavy vegetation causes velocity reduction through areas of the reservoir allowing deposition of sediment. A greater portion of the sediment delivered to the reservoir passes through the reservoir during periods of high flows, being kept in suspension by high velocities and carried over the dam. It has been calculated that during the spring months of March and April, the trap efficiency, or the proportion of suspended material the reservoir retains rather than passes is as low as 60\%. This is due to the high flows resulting from the large watershed. Increased vegetation in the upper reaches of the reservoir, however, will substantially reduce the velocity in that region and increase the trap efficiency throughout the year, increasing sedimentation. The trap efficiency of the Watervilet Reservoir presently ranges from a calculated 608 in March and April to a high of 928 in August. This coefficient can be expected to increase and hence significantly increase the sedimentation rate of the reservoir if steps are not taken to maintain the reservoir free from vegetation.

The third possible future impact involves the changing of the method of operation of the reservoir. This will be further discussed in the analysis and report for the Normanskill Hydroelectric Facility. As presently operated, the reservoir levels throughout the year do not vary greatly. In a typical year, the water surface of the reservoir may drop no more than 5 feet below 
the normal high water elevation at the top of the flashboards. Should the operating rules of the reservoir be modified to allow increased withdrawals for water supply or increased withdrawals from reservoir pool storage for hydraulic pumping or for generating hydroelectric power, the pool elevations might be significantly lowered. This would occur, in the latter case, if discretionary use were made of the storage pool capacity available in the reservoir for hydroelectric generation. Increased water supply demands would affect water levels since water supply would be taken on an annual basis and would draw from this pooled storage capacity regardless of water level. Wide fluctuations in the reservoir water surface elevation will affect both the amount of sediment in the reservoir and the distribution of the sedimentation in the reservoir. As presently operated, the reservoir is assumed to have a Type II reservoir depth versus capacity configuration as defined by the U.S. Bureau of Reclamation. This type of reservoir has been empirically determined to build up sediment and distribute sediment in certain patterns. With the water surface levels fluctuating greatly, these patterns associated with Type II reservoirs will change. Further analysis of these changes will be accomplished in the Hydroelectric Facility Report.

\section{Conclusions}

The following conclusions are offered for each module of the hydrologic analysis:

Data Base - No significantly useful stream tiow data is available from the historical record prior to 1967 . The meager data available was used as a guide and historical reference but could not be used analytically. An excellent stream flow data base was available from U.S.G.S. for the period 1967 to 1977 for the Normans $\mathrm{Kill}$ and the Hunger Kill. Excellent data relating to land use and soil conservation in the watershed is available and was used extensively in this analysis. 
Physical Survey - The photogrammetric examination of the existing reservoir shoreline coincides closely with that depicted by Solomon, Norcross and Keis. Deviations from the original shoreline occur at points of bank erosion. The limited depth soundings taken correlate closely with predicted volumetric losses due to sedimentation. The composition of core samples retrieved from the resevoir sediments is typical for reservoirs of this type and size.

Stream Flow Synthesis - A long-term ( 500 year trace) record of stream flow has been stochastically developed. It is believed to be an accurate representer of stream flow conditions for low flow considerations. The response of the model for high flow (flood) conditions has not been determined. Furthermore, the model can be recalibrated and its accuracy improved with the further stream flow data expected from the existing U.S.G.S. gauging stations. This stream flow model will be used with modification and refinement as the cornerstone of the hydroelectric generating scheme model and operating system.

Soil Loss - The watershed soil loss is termed "excessive" by the United States Soil Conservation Service as it relates to agricultural practices. However, the soil loss and resulting reservoir sedimentation are typical for a watershed of this type and land use. A further definition of the soil loss problem will be forthcoming in 1979 when the Soil Conservation Service has completed a Special Study under the Coopeeative River Basin Plan for the Normans Kill watershed. This data will be of use for conservation planning but it is not expected to materially affect the capacity or. yield determinations of this report.

Capacity Determination - The several methods used in determination of the existing physical capacity (volume) of the reservoir yield results of similar magnitude. With the reservoir elevation at 259 (full reservoir) taken as a reference, the available active storage calculated by Solomon, Norcross \& Keis in 1916 was between 1320 and 1690 million gallons with 1530 million gallons as the mean capacity (Figure 5). Decrementing this mean capacity for calculated sedimentation, the usable storage is 1450 
miliion gallons at the present time (Figure 6). Based on spot soundings, the capacity is determined to be 1360 million gallons. It is this figure (1360 mg), thought to be conservative, that is used for determination of the probable water supply yield of the impoundment.

Yield Analysis - The "maximum yield" of the reservoir for water supply purposes is defined as that amount which can be continuously witharawn with a specified likelihood of failure. The definition of failure is an empty reservoir. The maximum amount of drawdown (lowering) of the water surface is limited by the physical position of the water intake at the reservoir.

Specifically, for the watervliet Reservoir, as depicted on Figure 8 , the safe yield with a 99.88 reliability 10.28 probability of failure) is 12 million gallons per day. A 0.28 probability of failure is one year in 500 years. Similarly, the safe yield at 988 reliability (one year in 50) is $14.3 \mathrm{mgd}$.

It should be understood that the utilization of maximum yield does not imply available supply of a consistent quality of water, since extreme drawdown conditions will certainly affect water quality adversely.

Discussion of Error - Sources of error pertinent to this. analysis have been identified and minimized to the greatest extent possible. Further adjustment and refinement of the reservoir model deveoped should be directed in part toward refinements in accuracy of the model at high flows.

Future Impacts - There are considerable future impacts upon the Watervliet Reservoir. An impact of prime importance is the further intensification of land development. Establishment of watershed rules and regulations should be undertaken with the cooperation of the Town of Guilderland. The quality and quantity of water from this impoundment are related to land use in the watershed. The reservoir is a capital asset with an economic life dependent upon the proper operation and maintenance of the facility. As a man-made facility interfacing with the natural forces of eutrophication and sedimentation, the reservoir can serve its intended uses only through man's intelligent and corisidered intervention. 


\section{STREAM FLOW SYNTHESIS}

$\underline{\boldsymbol{\alpha}}$

\section{PROBABLE YIELD ANALYSIS METHODOLOGY}

The relatively short period of available historical records made direct interpretation of stream flow records ineffective in assessing a probability distribution of available reservoir yield: The statistical correlation between rainfall records and watershed runoff records was found to be poor and a generation scheme based upon the ample rainfall records was also ruled out. Likewise, correlation beteen the reservoir watershed runoff and other stream flows for which longer historical records were available was inconclusive and a streamflow analysis by comparative hydrology was not used.

It was therefore decided that a flow simulation scheme would be used in the study so that a lengthy synthetic record of reservoir watershed yield could be available for analysis. Although not an actual historical record, the model would statistically resemble the comparatively short historical record and have the distinct advantage of adequate length and variety.

The reservoir watershed runoff model that was selected for simulation is a Markov model having the statistical properties that resulting flows have a deterministic component and a random component. It is the property of a normal Markov process that elements in a generated series have correlation to elements preceding them. In this instance, flows representing monthly mean reservoir watershed runoffs were generated and the persistence correlation was limited to one month, the simplest form of Markovian flow model. That is to say, the model assumes that the entire influence of the past on the current flow value is reflected in the previous flow value. The deterministic component of monthly flows, therefore, is a Iinear auto-regressive form of the historical sample's monthly mean plus the persistence element which correlates 
the model's previous month's departure from its sample monthly mean to the current month's departure in proportion to the standard deviation of the historical monthly flows. The random component of monthly flows is computed using a random standard normal deviate series. This series of random numbers has the statistical properties of a normal frequency distribution with a zero mean and unit standard deviation. The magnitude of the random component is a function of sequential random numbers from the series, the correlation to the previous month's flow and the standard deviation of the historical flows for that month. The normal distribution of the random standard deviate series imparts to the synthetic flow sequence also a normal distribution. Likewise, the standard deviation of the historical monthly flows is preserved in the model by factoring it into the computation. A discussion of how the type of frequency distribution was determined and the means of arriving at the historical record flows is described in subsequent paragraphs. The generation of the random normal standard deviate series employed the computer's capability of generating random numbers having a uniform distribution. A series of 600 numbers was generated in each sequence by the computer program for use in computation of 50 years or 600 months of flow, since flow sequences were generated and stored by the computer program in 50-year blocks. Six hundred totals each of six uniformly distributed random numbers ranging from 1 to 1,000 are first determined. These random totals have an approximately normal distribution. subtracting the mean value of these 600 normally distributed random totals from each total and then dividing each element of the resulting series by the standard deviation of the series, we are left with a series of 600 random numbers having a frequency distribution which is approximately normal and having a zero mean and unit standard deviation.

A digital computer was used extensively in the computation. The computer was equipped with approximately 42,000 bytes of available active memory and was programmed in APL programming language. A 500-year flow sequence was generated in 50-year 
increments containing 600 monthly values each and was stored on magnetic tape files requiring 5,000 byte blocks each of floating point storage. The generation of 500 years of synthetic flow records required approximately four hours of running time exclusive of determination of model statistical parameters based upon analysis of historical records.

The following is an outline of all sequential steps taken and of the most important assumptions made during the progress of the study in order to accomplish the probabilistic determination of water supply yield at the existing Watervliet Reservoir:

1. United States Geological. Survey Water Resources Data was researched in order to obtain runoff records for the entire period of record by months from October 1967 to September 1977 for the 131 square mile drainage area of the Normans $\mathrm{Kill}$ measured downstream from the dam in Guilderland near state Highway 155.

2. Similarly, recorded flows were gathered for the same period for the eight square mile drainage area for the Hunger Kill, tributary to the Normans Kill upstream from the Normans Kill gauging station and downstream from the Watervliet Dam.

3. The operator's $\log$ at the Watervliet pumping station was researched for the entire period of all notations regarding water level elevations in the reservoir and other miscellaneous information regarding water supply usage and turbine operation. This information was correlated with monthly stream flows for the record period.

4. The net monthly mean reservoir inflows for the ten-year period were computed based upon the recorded flows in the Normans Kill downstream from the dam. Additions to the basic flow in the Normans Kill were made for water diverted from the reservoir for the City of watervliet in the Town of Guilderland for water supply purposes and also for increases in reservoir storage due to increased water level in the reservoir from month to month. Subtractions were made from the basic flow for recorded stream flows in the Hunger Kill as well as for that portion of the Normans Kill drainage area downstream from the dam and upstream from the gauging station. The assumption that this downstream area contributed $9.82 \%$ $(11 / 112)$ as much runoff as the reservoir watershed was used. 
5. Sample frequency distributions for mean monthly runoffs and the natural logarithms of these runoffs were plotted in order to determine a representative frequency distribution of stream flows for each month for use in stochastically generating simulated monthly mean runoff sequences. Although there were only ten historical values for each month on which to base the choice, the assumption of a log-normal frequency distribution appeared to adequately represent the historical record of reservoir inflows.

6. Lag-l statistical serial correlation coefficients were determined for the historical ten-year record correlating the logs of the mean monthly inflows for a given month and those for its preceding month. This correlation is a measure of the tendency in the historical sample, and thereafter in the stochastically simulated model, for a departure in a given month from its sample monthly mean to be repeated in the following month by a similar departure from its monthly mean. The samples referenced in this case are the natural logs of the historical inflows.

7. The computer program was then developed to simulate (generate synthetic values of) monthly mean reservoir runoffs for an extended time period using a stochastic Markovian Lag-One seasonal model assuming log normality of distribution. A stream flow synthesis for a 500-year period was thereby produced resulting in a 6000-month synthetic record of monthly reservoir inflows which had the statistical properties that the frequency distribution of its lngarithms was normal, and the means, standard deviations, and Lag-One serial correlation coefficients of the logarithms of the monthly mean flows matched that of the historical record. The synthesized sample was otherwise completely random. The Lag-One serial correlation coefficients correlating logarithms of the mean monthly flow for January through December with its preceding month that were determined and used in the simulation were $.73, .50,-.29$, $.09, .18, .62, .67, .20, .52, .82, .26$, and .15 , respectively.

8. Using the 6000-month record of inflow simulation, a reservoir operating model using computer was run assuming a uniform monthly draft rate taken from the reservoir for several sample draft 
rates. The amount of reservoir storage deficit below full for each of the 6000. months at each draft rate was tabulated. In each case, the reservoir was found to have refilled by March so that no operating deficits below full reservoir were carried through the following year.

9. From the deficit tabulation at various draft rates, the frequency distribution of reservoir storage deficits was determined versus uniform draft rate from the reservoir. That is, the number of times in 500 years of stream flow synthesis that a storage volume requirement would be exceeded at draft rates of $6,10,14$ and $18{ }^{\circ} \mathrm{mgd}$ was tabulated and plotted.

10. Finally, the reservoir deficit analysis was plotted in a form which demonstrated the reliability of safe, uniform yield of the simulated water supply versus the reservoir capacity required to realize this yield. A reliability of 998 implies that the required reservoir capacity would be exceeded once in 100 years at the indicated draft rate, and the 99.88 reliability implies once in 500 years.

11. This probablistic approach must be recognized as representing the reliability of a given yield in the 500-year reservoir streamflow simulation. The validity and accuracy of the risk analysis are naturally dependent upon the validity of the various assumptions hereinbefore explained. We are obviously Iimited by the brevity of the historical record to verify assumptions of the $\log$ normalcy and to accurately determine the long-term mean, standard deviation, and Lag-One monthly correlations and the validity that the historic record period is representative statistically of much longer period. We feel that for low flow considerations of this report, that the period is sufficiently representative for values for mean and standard deviations to yield proper results. The most significant factor in determining safe yield of the reservoir is the probability that extreme low flows in a given month will be repeated for $3,4,5$ or even 6 months in succession, and the most significant factor in this determination is 
the Lag-one monthly serial correlation of flows. The synthesized flows are extremely random with only correlation coefficients influencing the persistence or tendency for low flows to follow low flows.

The alternative to this method of analysis is comparative stream flow hydrology from a similar stream with a long record of historical flows, and we feel that the necessary assumptions in utilizing such an alternative method are likely to be significantly inaccurate or inappropriate.

\section{APPENDIX B}

\section{SOIL LOSS DETERMINATION METHODOLOGY}

The soil loss analysis was based upon computations for sheet erosion and stream bank erosion for the entire Normans Kill watershed. As defined by the soil conservation service, sheet erosion includes geological, natural, normal, rill, sheet and splash erosion. Gully and wind erosion are considered insignificant and are not included in this analysis.

The analysis uses the Universal Soil Loss Formula developed by the U.S. Department of Agriculture. Raw data compiled by the Albany and Schenectady County soil Conservation Service offices was obtained for purposes of our analysis. The raw data includes estimations and quantification of parameters on an area basis for numerous capability subclasses and dominant soil types. This raw data includes " $K$ " factor estimation (soil erodibility), slope length, slope, and "C" factor (conservation practice). Since the Albany County raw data includes portions of the Normans Rill (watershed No. 177) basin downstream from the Watervliet impoundment, a disaggregation of those areas downstream was made. The disaggregation was done on a small scale (several acres) basis using New York State Land Use Natural Resources (LUNR) inventory mapping. Upon a base map of the sub-basin area on which " $K$ " factors were noted, overlay maps were superimposed. These overlay maps consisted of (I) capability subclass and dominant soil 
characteristics mapping, and (2) predominant land use (IUNR data). From this disaggregation, a tabulataion corresponding to the total Albany County Normans kill sub-basin tabulation was prepared. Soil loss for the watershed tributary to the Watervliet Reservoir was defined as Albany County total plus Schenectady County total minus Albany County disaggregation portion. Spot verification was made of both Albany and Schenectady county raw data to verify the validity of the data. Certain correction factors for non-contributing areas such as ponds, lakes and rivers were made to the raw data. Arithmetic manipulation of the resulting data matrix yielded a quantification of the total tons per year for the watershed. tributary to the Watervliet Reservoir. A separate calculation for stream bank erosion was performed using stream bank erosion quantities determined by soil Conservation Service. Total annual. soil loss was then proportioned for each month of the year based upon a percentage of precipitation falling during that month. Minor correction factors for winter months were made. A sediment delivery ratio was then applied to these monthly figures. The sediment delivery ratio is an empirical ratio based upon watershed size that $y$ ields the percentage of soil loss actually entering and transported by the major stream courses in a watershed. The result of the soil loss analysis is a determination of the total tons delivered to the entrance of the reservoir for each month of the year.

\section{APPENDIX C}

\section{SEDIMENTATION ANALYSIS METHODOLOGY}

Using the data developed under the soil loss portion of the analysis, a sedimentation rate, or number of tons delivered to the reservoir on a monthly basis, was determined. Trap efficiencies for the reservoir were calculated similarly on a monthly basis. This trap efficiency was calculated on a monthly basis because of the wide variation in stream flow from month to month. The trap efficiency determination was developed by Brune [Ref. 31]. It is an 
empirical coefficient whose value is a function of the ratio of reservoir volume to mean flow. Using the twelve monthly trap efficiencies thus calculated, a value for the number of tons retained in the reservoir for each month of the year was obtained. These twelve values were summed, giving an annual sedimentation load in tons per year.

Conversion of this annual sedimentation rate into expected volumetric changes was then performed. The density of soil deposited in the reservoir is dependent upon the constituency of the soil. General guidelines for particular watersheds and reservoir types relating to percentages of silt, clay, etc. were obtained. As a check on these empirically developed values, field sampling of in-place sediments was performed, using a pfleder sediment sampler. The recovered core samples were visually classified and tested for moisture content, specific gravity and grain size distribution. The determined values were then used in standard soil state calculations to derive unit weights. The unit weights and specific densities of the samples taken were then manipulated to yield a sediment density calculated to be 62.7 pounds per cubic foot. Since further consolidation of sedimentation occurs over a time, an average weight at the present time ( $T=62$ years) was determined using the relationship:

$$
W_{T}=W_{T}+.4343 k\left[\frac{T}{T-1}\left(\log _{e} T-1\right)\right]
$$

where $W_{T}$ equals weight at $T$ years

$w_{1}$ equals initial weight

$K$ equals constant based on a grain size analysis of the sediment.

Further calculation to determine the weight at 100 years was similarly made. By dividing the annual sediment retained in the reservoir by these average weights, the volume displaced by sediment was thus determined. 
Distribution of this calculated sediment volume over all elevations of the reservoir was made using the Area-Increment Procedure. This procedure is basically an iterative procedure for distributing the volume loss proportionately over all elevations.

\section{APPENDIX D}

\section{PHYSICAL SURVEY METHODOLOGY}

A physical survey was performed on the reservoir to yield in-place data relating to sedimentation and also for verification of actual depths. The methodology for the sediment determination is discussed in Appendix C "Sedimentation Analysis Methodology". Spot depth soundings were performed for verification of actual volume loss by the following method:

The reservoir was divided into six component areas. These component areas were generally wide areas separated by narrow channels. Across each of these areas and across several of the channels range lines were established. These range lines consisted of a base line extending from one side of the reservoir to the other. The base lines were documented so that they can be relocated and checked in the future for additional sedimentation calculations. Using an electronic distance meter (EDM), located on one shore, the sounding boat completed a traverse to the other shore. At predetermined intervals soundings were made using a line and sinker method and the distance to the sounding point was measured by the EDM. This field survey took place during a period when the elevation of the reservoir was below full capacity (flash board height) so no head water calculations were necessary. In addition, the entire field survey for soundings was performed on a calm day so that no wave-induced elevation errors occurred. Pool elevation was then transferred to a physical benchmark located at the dam. Using the spot hydrographic data thus obtained, cross-sections for each range were plotted and were compared with 
cross-sections derived from the original topographic survey performed by Solomon, Norcross \& Keis, Engineers. These cross-sections are presented as Figures 3 and 4.

\section{APPENDIX E}

\section{CAPACITY DETERMINATION METHODOLOGY}

The physical capacity of the reservoir was determined by several methods. Due to the limited financial resources and due to other logical considerations, a complete field topographic or hydrographic survey could not be made. Capacity versus elevation curves were developed for three different conditions.

The first condition was simply a capacity versus elevation curve using Keis' original topographic analysis. A curve was developed utilizing Keis' topographic values exactly as shown on the original mapping. Two additional curves were also developed using these contours but assuming a possible error of plus or minus one-half contour interval or $2-1 / 2$ feet. The three curves thus developed were referred to as "Keis' mean", "Keis' high", and "Keis' low" capacity curves (Figure 5).

The second condition is a graphical representation of capacity versus elevation using the original "Keis' mean" contour data decremented by sedimentation calculations to yield a reduced capacity curve projected for 62 years (at present time) and for 100 years (expected capacity in the year 2016).

The third condition for capacity determination utilized the physical hydrographic data obtained over the five sample ranges. Each range was compared to a calculated cross section derived from Keis' original topographic survey. A percentage change was calculated for each elevation and the resulting gross reductions by cross-section were then tallied proportionally to reservoir areas in relation to the ranges. A composite capacity curve was thus developed yielding cumulative volumes vs. elevations. The results of this condition for volumetric determination are presented in Figure 7. 
It was found that the capacity curve determined by soil loss, Figure 6, and the capacity/elevation curve developed as a result of cross-sectioning, Figure 7, correspond closely. Also noted on each of the graphs is the minimum water surface elevation necessary to supply water to the City of Watervliet via the intake rack located on the Watervliet dam as well as to the Town of Guilderland via the lowest intake opening on the Guilderland water Pumping Station and intake structure. Usable capacity is thus defined as the volume of, water stored above elevation 240 in the reservoir. Storage below elevation 240 is termed dead storage and is unavailable for draft. 


\section{APPENDIX F}

- Low occurrence

$M$ - Medium

$H-\mathrm{High}$

Analysis Module

U.S.G.S. Flow Data
Operator Logs
S.C.S. Soil Loss
Data "K" factor
"C"
"R" "P" $"$
Area
Rainfall
Land Use Data (LUNR)

Photogrammetric evaluation Sediment sampling

Hydrographic survey

Historic record

Frequency distribution

Computational method

\section{Errors}

$\mathrm{H}$

L

L

M

$L$

\section{RECOGNIZED ERROR - OCCURRENCE}

$\begin{array}{ccc}\text { Judgmental } & \frac{\text { Observational }}{\text { L }} & \frac{\text { Analytical }}{M} \\ \text { L } & M & \text { N.A. } \\ & & \\ \text { L } & \text { N.A. } & \text { L } \\ \text { M } & \text { N.A. } & \text { N.A. } \\ \text { L } & \text { N.A. } & \text { L } \\ \text { M } & \text { N.A. } & \text { N.A. } \\ \text { L } & \text { N.A. } & \text { N.A. }\end{array}$

L L

L

$\mathrm{L}$

Multiple observations and small scale minimize errors. Most errors relate to resolution of non-structural (nonurban uses. For this study differentitation of the type not significant.

\begin{tabular}{|c|c|c|c|}
\hline N.A. & L & $L$ & \\
\hline $\mathrm{L}$ & $\mathrm{H}$ & L & $\begin{array}{l}\text { Multiple records, verifiable with } \\
\text { other data. }\end{array}$ \\
\hline $\mathbf{M}$ & L & L & $\begin{array}{l}\text { Selection of ranges sensitive to } \\
\text { judgmental error. }\end{array}$ \\
\hline
\end{tabular}

See data base above.

L

Multiple season madel minimizes error.
Numerous data points minimize sensitivity. Data verifiable by other sources.

Multiple observers minimize errors.
Selection of ranges sensitive to 
APPENDIX F

\section{RECOGNIZED ERROR - OCCURRENCE}

\section{Analysis Module}

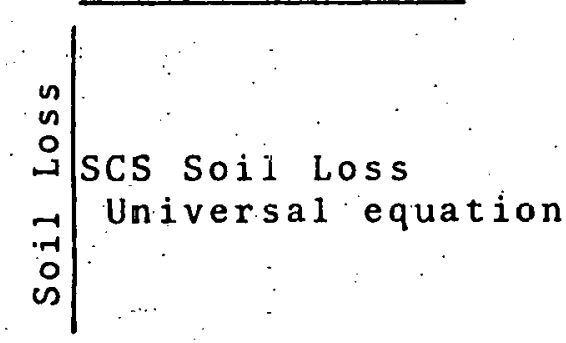

Sediment distribution

这窎

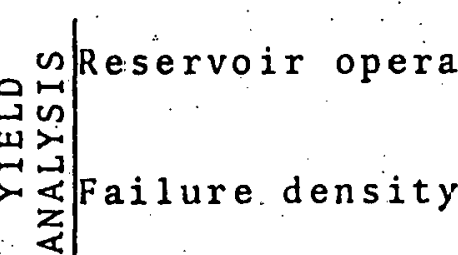

\section{Errors}

\section{Judgmental Observational Analytical}

N.A

L

N.A.

N.A.

M

N.A.

L

L

L

\section{Comments}

N.A .

$M$

Universal model is empirical.

Computational algorithms use digital computer and are based on simple arithmetic computations. 

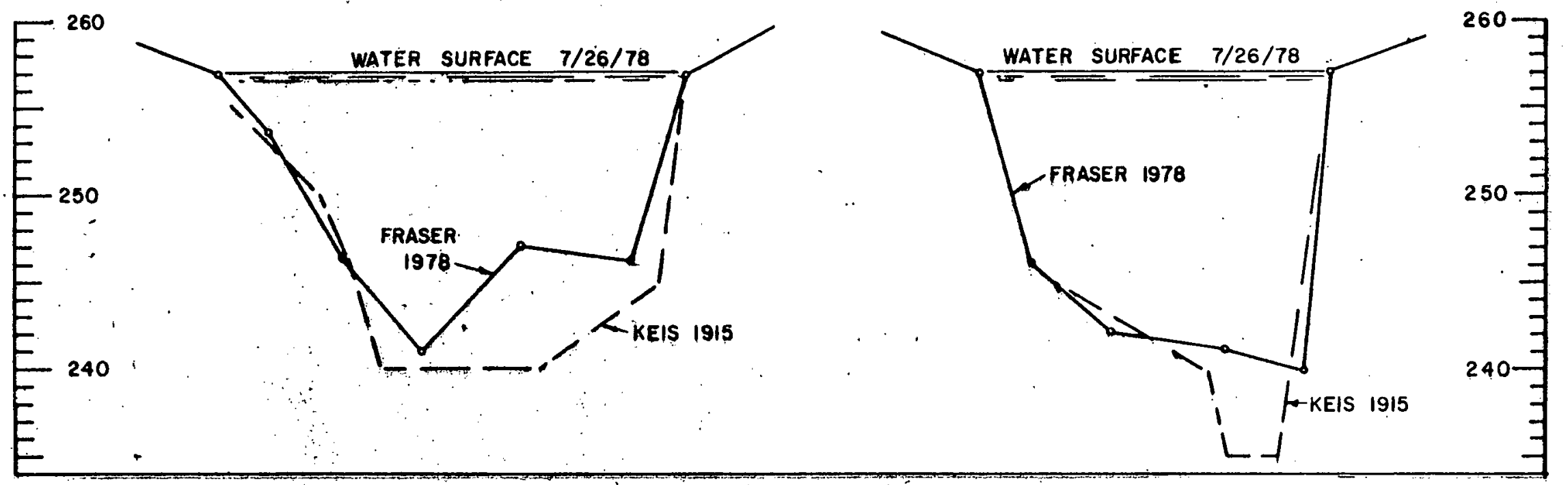

RANGE NUMBEP TWO

RANGE NUMBER THREE

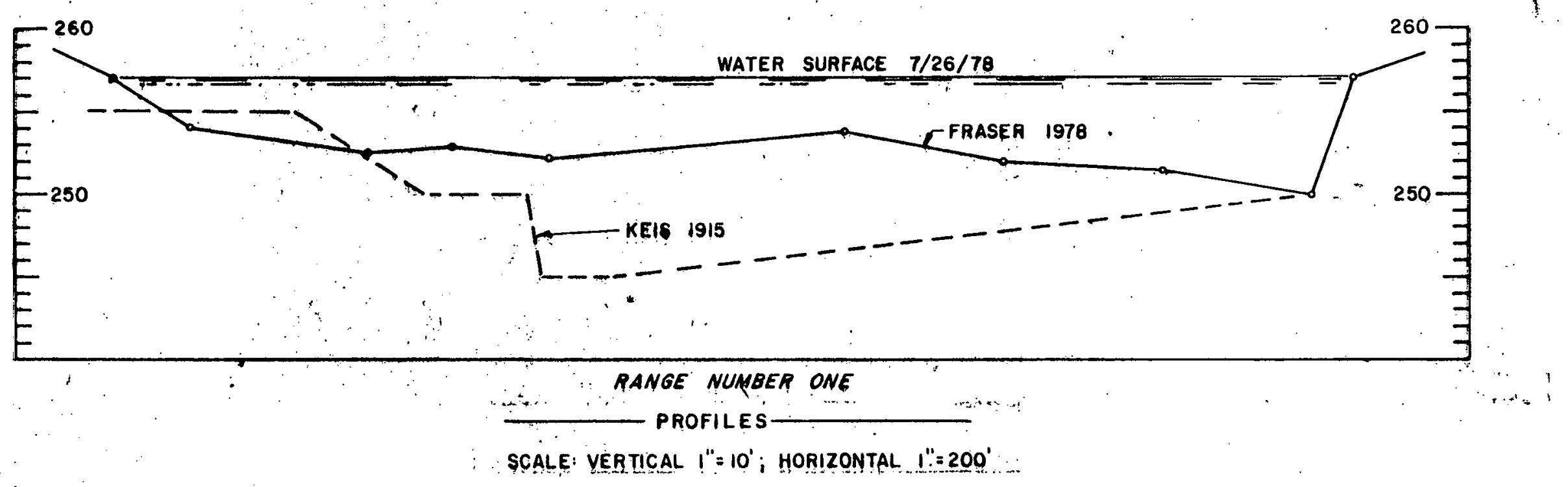




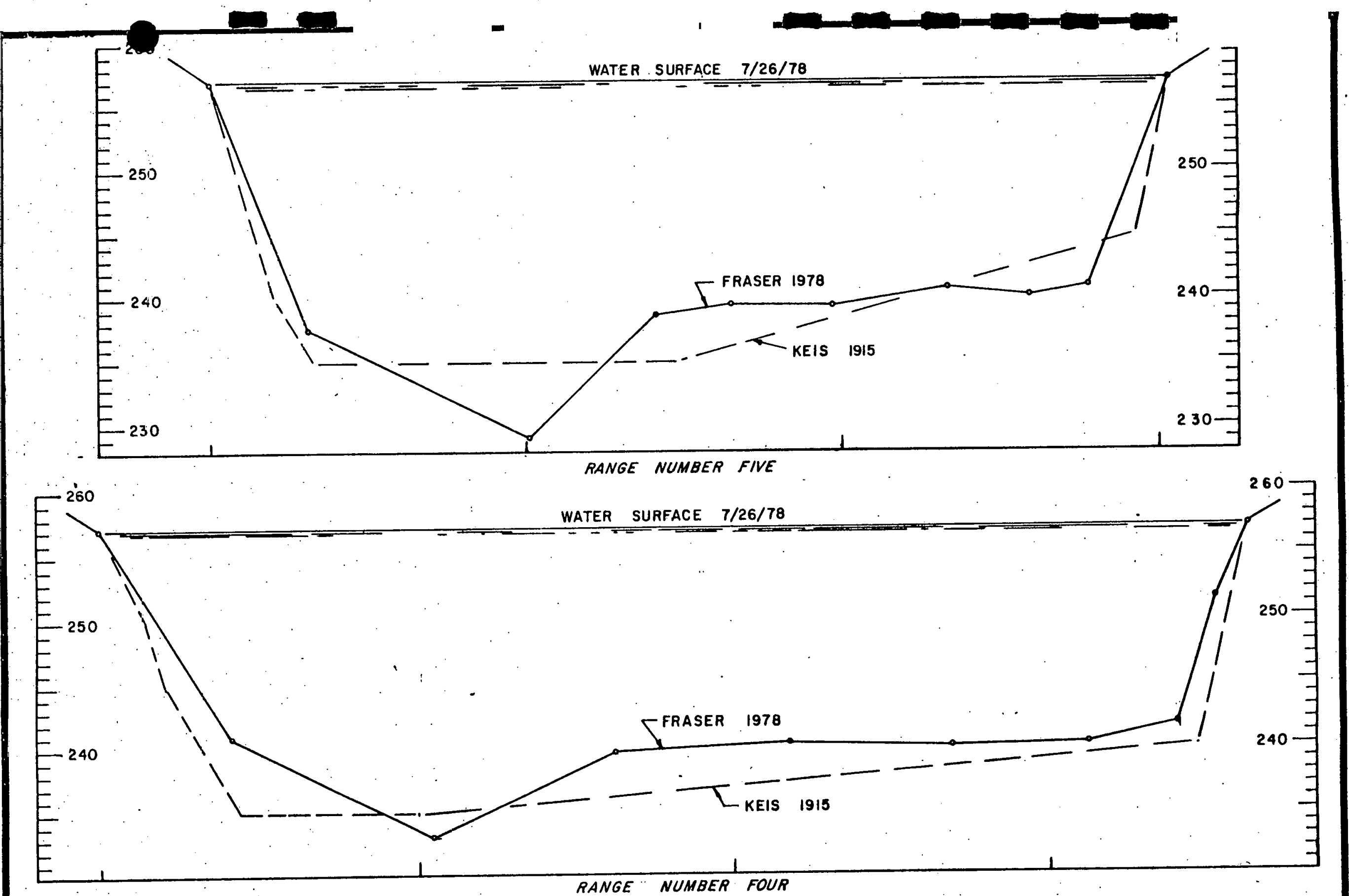

PROFILES -

SCALE: VERTICAL $1 "=10^{\circ}$ : HORIZONTAL $1 "=200^{\circ}$

NORMANSKILL HYDROELECTRIC FACILITY RESERVOIR PROFILES

FEASIBILITYY ASSESSMENT-EW-78-F-07-1765 


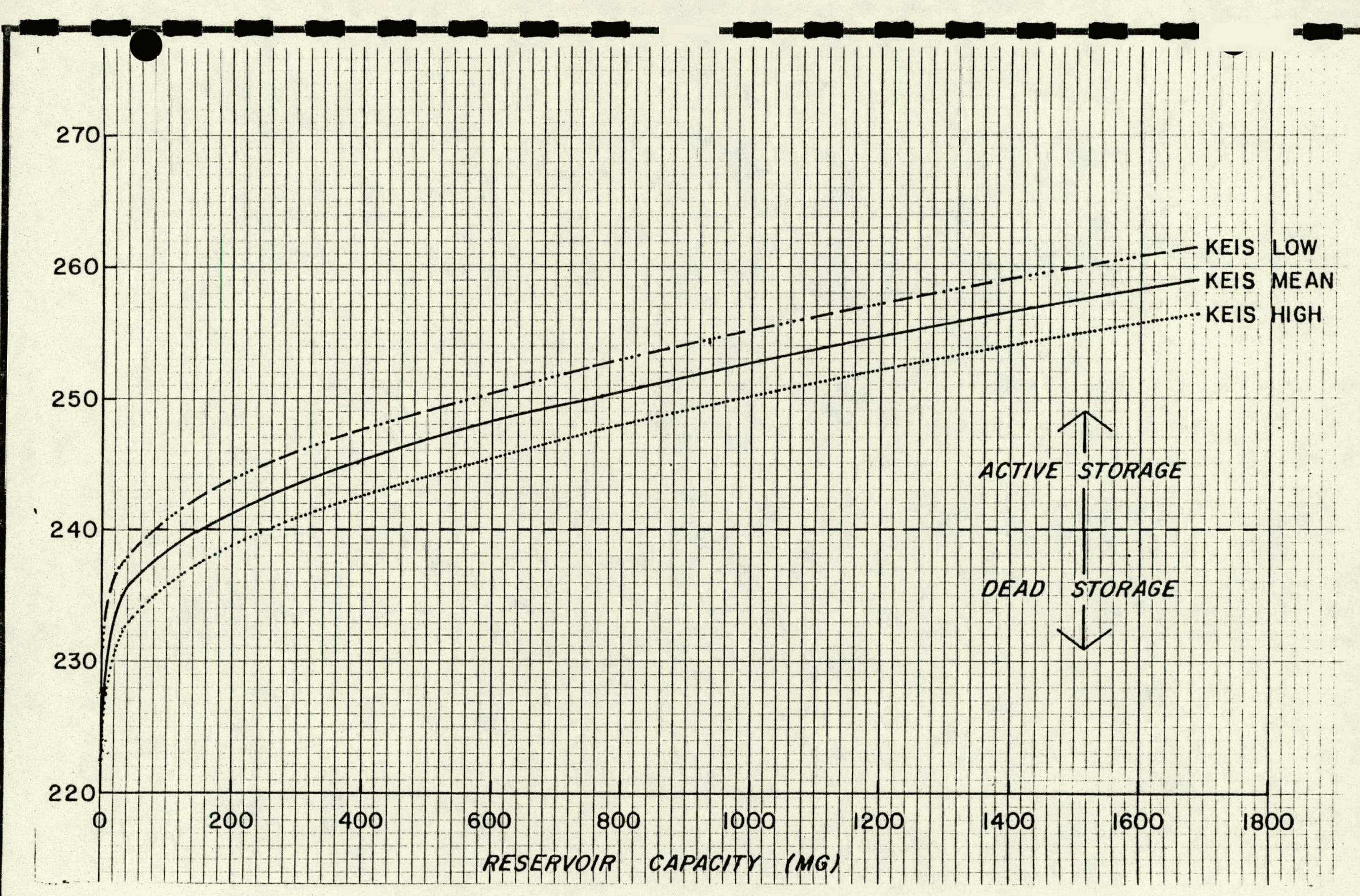

NORMANSKILL HYDROELECTRIC FACILITY

FEASIBILITY ASSESSMENT-EW-78-F-07-1765

RES. CAPY.

vs SURFACE ELEV. 


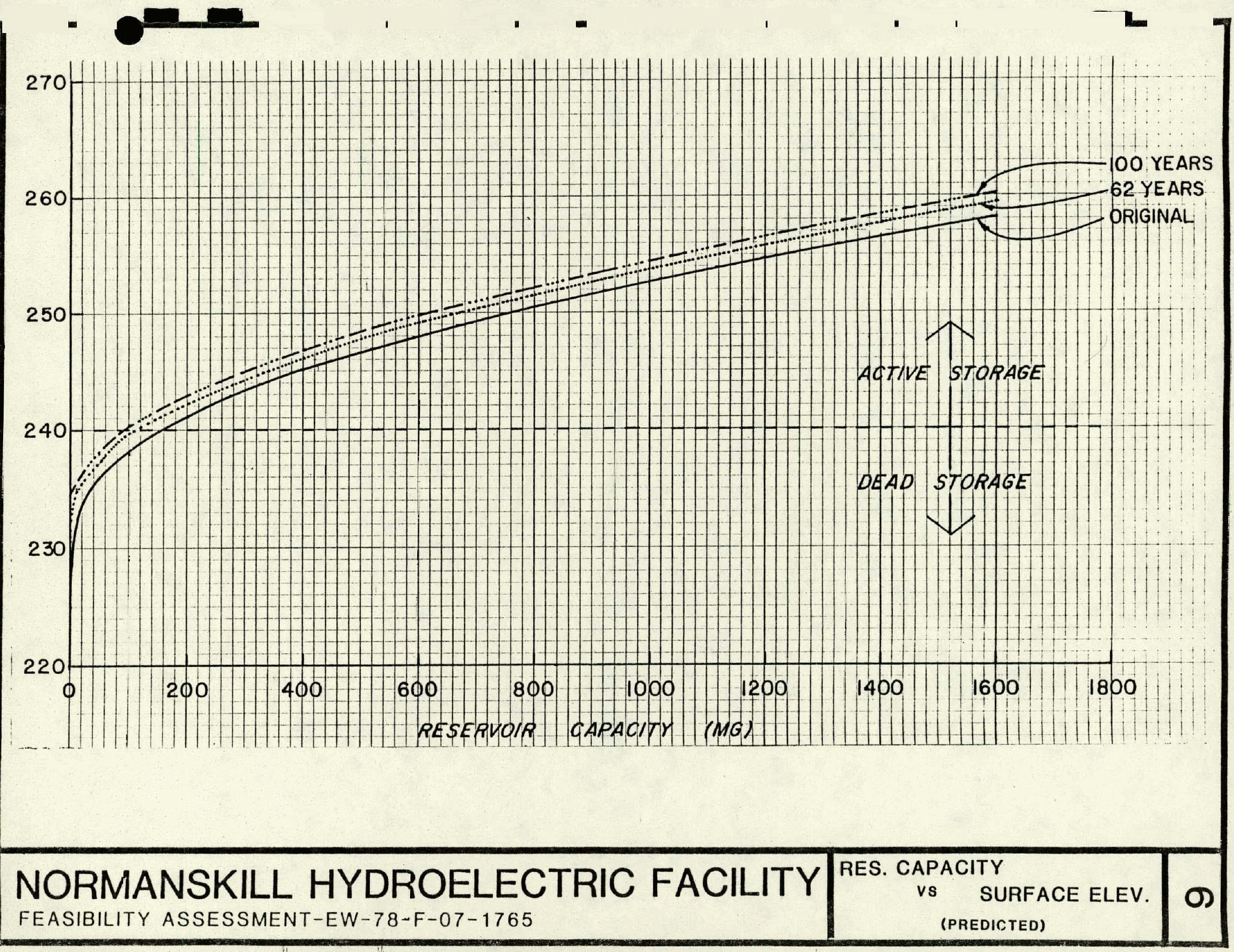




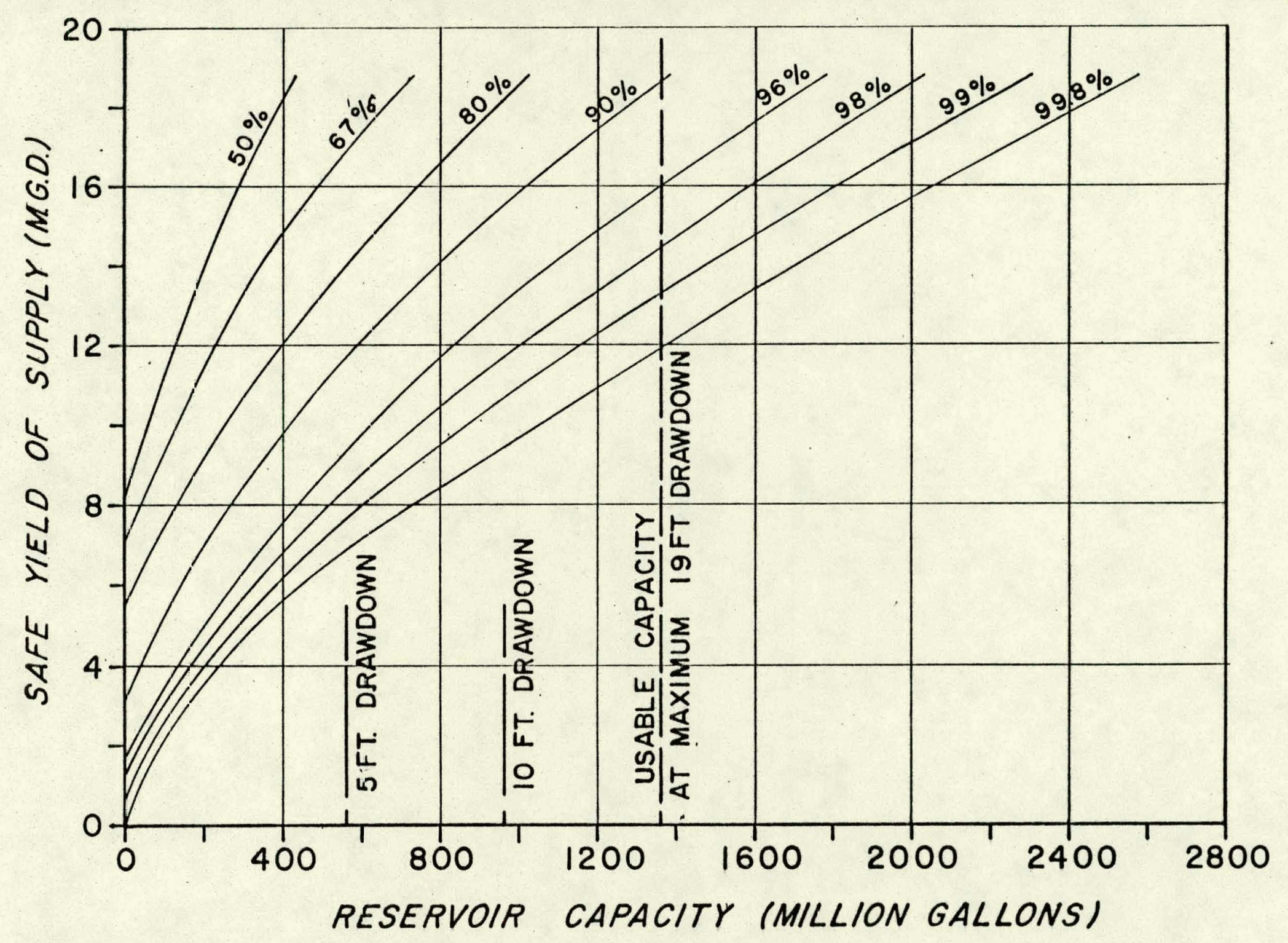

RELIABILITY OF WATER SUPPLY YIELD VS. RESERVOIR CAPACITY BASED ON 500 YEAR STOCHASTIC RUN-OFF MODEL 


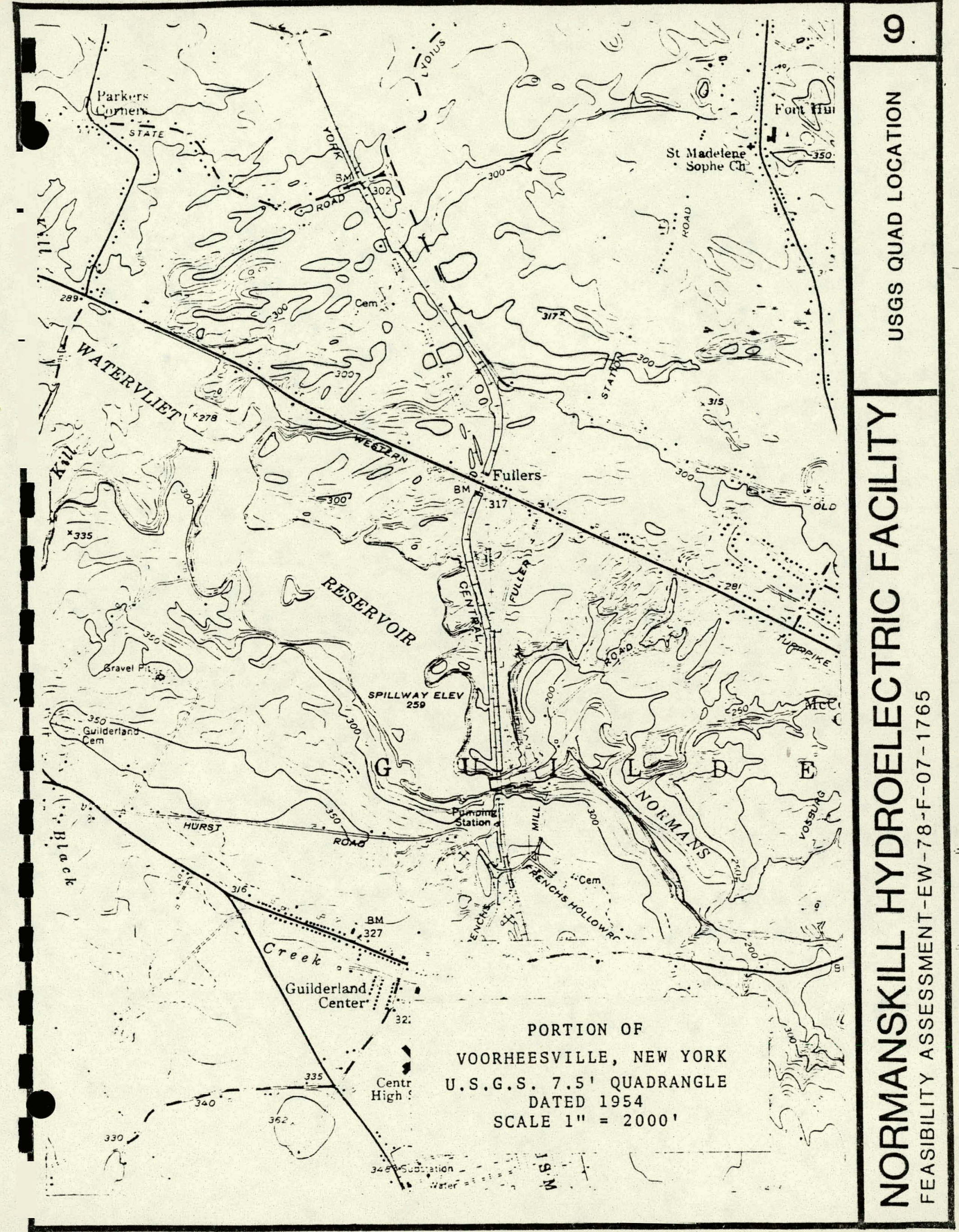




\section{HYDROLOGIC - SEGMENT TWO}

\section{Data Base}

Segment one of the hydrologic phase investigated the various elements of the existing Normans kill impoundment as it relates to the community as a continuing source of municipal water supply. Therein, an attempt was made to quantify certain of the physical aspects of the reservoir such as magnitude and rate of siltation of the original impoundment, its remaining usable capacity and the effects of land use and age on the water quality for present and future users of the resource.

The water supply yield of such a water resource cannot be quantified as a single fixed number. The water originates in the hydrologic cycle in nature and enters the impoundment as watershed runoff from rainfall. It is affected by evaporation, transpiration, soil absorption and percolation, freezing and melting and a myriad of other factors. The quantity of runoff is largely a natural phenomenon and is partially random and partially predictable. Because of the random component of watershed runoff, it is misleading to try to describe the yield of a water resource without some corresponding explanation of the probability that a yield can be relied upon.

Our approach to determining the water supply yield of the Normans Kill impoundment recognized the tremendous variation in stream flow and the extreme importance of the storage capacity in the yield determination. Figure 8 (Chapter 2) displays the relationship which we concluded exists between the safe yield of the impoundment and the probability or confidence level that the impoundment will not be depleted by more than the capacity shown in millions of gallons.

Limited by the fact that the 10 year daily record of stream flow for the Normans Kill by the United states Geological survey was gathered downstream from the dam, this record was of little assistance in determining the flow into the impoundment on a daily basis since reservoir levels on a daily basis were unknown. With 
the help of the log maintained by the operator of the existing water pumping station at the site, we were able to determine with reasonable accuracy the reservoir levels at the beginning of each month auring the period of record and thereby to determine the monthly mean runoff available at the impoundment during that period. It is important hereafter to keep in mind that the actual stream flow data base for this water resource facility is therefore limited to 10 years of monthly mean flows from the period commencing October 1967 through september 1977.

Knowing that the runoff record for a 10 year period would not contain the worst combination of low flows which could be expected over a much longer period, we adopted an approach described in Chapter 2 to expand the data base. statistically to enhance its usefulness. The statistical methods are described in considerable detail in Appendix A of Chapter 2. In the course of developing Chapter 2 of this report, a data base of 500 years of monthly mean watershed runoff values was derived by simulation. The simulated data retained all the important deterministic statistical

parameters of the shorter historical record while also being capable of displaying a randomness found in natural phenomena.

The relationship displayed in Figure 8 is based on the entire data base of 6,000 monthly values for the 500 year simulation. A 500 year data base was required to develop the accuracy displayed in the probability curves. The water supply aspects of the Normans Kill impoundment concentrated on the lower range of water supply yield rate and a very high range of confidence. As one can see from Figure 7, the confidence level that a given drawndown will not be exceeded drops dramatically as one increases the yield of the reservoir.

In this segment of the report, we must determine the selection of the optimal design configuration of a hydroelectric generating facility and must answer the question whether such optimal configuration is economically feasible to construct. In contrast to the maximum reliability demanded for the water supply yield, it is not a prerequisite that a hydroelectric generating facility operate 
continuously. The criteria for optimal selection of the hydroelectric facility configuration is dependent on economic choices based on maximizing benefits or return on investment in the form of sale of power or reduction of current power costs, and simultaneously minimizing the costs necessary to achieve those benefits.

Determination of power producing capability is a function of a large number of variables relating to machine configurations, location, etc., but it is initially dependent on the quantity of water available to be committed to this purpose. It is our desired purpose to be able to express the benefit parameters for various configurations which will be investigated in terms of annual or monthly averages.

In determining such averages, it is a tempting but an erroneous oversimplification to utilize simple monthly or annual average runoff rates. " Power production is limited by the machine configuration to a fixed maximum rate while the quantity of water which is allocable to hydroelectric generation may be available at an extremely wide range of rates depending upon reservoir storage and stream flow. A stream flow sequence may be comprised of many different combinations which produce the same average flow but which. result in considerably divergent total energy output because of the random occurrence of flows which may be too great to be able to be utilized by a particular configuration. For this reason, the averages which we ultimately develop are in fact the result of mathematically modeling a given configuration through a long term sequence of simulated data and then determining the average or representative resulting parameter value for each month in the period, instead of utilizing a simple streamflow average. Economic benefits from power generation are likewise not determinable by prior averaging of stream flows or of turbine running times because of a discontinuous energy benefit rate structure. Inherent in this rate structure is the anomalie that the extreme high and low values of power production which contribute to an average value are in $f a c t$ accruing benefits at extremely different rates. 
A sufficiently large data base to give adequate variety to the data and to be consistent with the historical runoff record is required for use in the hydrologic computations for the hydroelectric generation study phase of this report. A 500-year flow simulation became available as a by-product of the segment one study. We have chosen to use only the first 50 year block of this 500-year record as being adequate for purposes of the hydroelectric feasibility assessment since the time required to perform the extremely high number of trial computations using a greater length of data than a 600 month sequence would be prohibitive and would not significantly alter the results.

Since our computations in this study are monthly in nature, we have prepared a series of graphs (Figures 11-22). to display certain aspects about the data base for each month as used in each segment of the study. Each graph represents the data for a particular calendar month. The data is displayed using a logarithmic vs. probability scale since it was determined as described in segment One, that the most representative distribution for the data points was log normal, or that the logarithms of the data points tended to have a normal distribution. The ten discrete data points represent a plotting of the historical record of flows determined by methods discussed in detail in Appendix A of the preceding chapter and arranged in such a way that each of the ten points taken in order of magnitude is plotted in the middle of its respective decade of cumulative probability of values being less than that value.

A distribution of values when plotted on logarithmic probability paper can be'considered to be log normal if it can be reasonably assumed that they tend to define a straight line. This assumption was in fact made and incorporated into the process of modeling the data. Line $A$ on each of Figures 11-22 depicts an idealized normal distribution statistically representative of the ten historical data points. The line crosses the median or 50 percentage point at a value whose logarithm is the mean of the 
logarithms of the ten data points. Two additional points on this line are then produced by computing the standard deviation of the logarithms of the ten values, adding and subtracting it from their mean and plotting the value whose logs are those numbers at points which are one standard deviation or $34.13 \%$ above or below the median respectively. The line thus determined is a representation of the distribution of data points using the mean and calculated standard deviation of the logs of the original data. The detailed computation as described in Appendix $A$ of the preceding chapter describes how the simulation process using the computer was completed. Line A therefore represents the true distribution of data points that would be produced for a simulation of infinite length for each month. Our 500 year simulation is of sufficient length to approximate this line extremely closely. As described in Appendix $A$, distributions for each month are not independent, but contain a factor which reproduces the correlation between subsequent months of the historical record into the simulated record.

As explained previously, it was determined that a 50 year. segment of the 500 year simulation was sufficient for the purposes of this segment of the study. The smaller subset of the 500 year sampling will naturally show some variations from the sample taken as a whole. Line $B$ on Figures $11-22$ is an idealized representation of the distribution of the data points in the 50 year simulation block. This line was produced in a manner similar to that described above using the mean and standard deviation of the logarithms of each element in the distribution. There are minor variations between the distribution of the 50 year subset and that of the 500 year sample, however, uniformity has been adhered to in using the 50 year synthetic record in this segment of the analysis. We feel that the reliability of our computation has been greatly enhanced through the use of this simulation technique and that the 50 year synthetic record reasonably represents and augments the limited 10 year historical record. 
We have discussed and presented individually the probability distribution for each calendar month, but have not conveyed a sense of continuity from one month to the next. Figure 23 displays the amount of monthly mean runoff available based on the io year historical record for each calendar month expressed in terms of the probability that such runoff rate will not be exceeded. We have presented this information with respect to probability levels of 10 , 30, 50, 70 and 90 percent where the 50 percent or median level is the most likely value to occur and there is an 80 percent probability that the monthly average value for any randomly selected month will fall between the 10 and 90 percent values. We have attempted to impart a continuity to these confidence levels by sketching continuous curves for each in such a way that integration of the area under the curve approximates the numerical integration of the discrete values taken as constants during each month. We do not imply that an actual occurrence at any level will be followed by one at the same level the following month. There are factors correlating the tendency of such persistence to exist which vary from month to month and which is detailed in Appendix A preceding. It is sometimes helpful to be aware of the fact that the mean or average value of a sample of flow values having a log normal distribution is not equal to the median or most likely occurrence. In any of the monthly distributions the mean is always higher due to the existence of less frequent but disproportionately higher flow rates.

We have indicated on each monthly graph of data base frequency distribution. (Figures 11-22). the mean value of the 50 monthly mean inflows to demonstrate distinction between the median and the mean for the 50-year simulation.

It is often of interest to relate the effectiveness of a water supply in terms of the mean annual runoff which enters it: The proportion of a stream's mean annual flow which can be utilized for water supply is primarily a function of the volume of storage capacity of the impoundment and the distribution of inflow occurrences, It is often desirable to develop a reliable use of at 
least $50 z$ of a stream's mean annual flow for water supply. To do so, however, might require a usable impoundment storage volume of approximately $30 \%$ of the total annual runoff. The first 50 years of the 500 year monthly mean inflow simulation has been utilized in segment Two of the hydrologic phase of this report as its data base. The mean annual flow of this 50 year sample is 4,852 billion cubic feet or 36,290 billion qallons. This value is 2.378 higher than the mean of the 10-year historical record. This fact can be attributed to the existence of infrequently occurring but disproportionately higher values in the larger sample which is to be expected. This total mean annual flow is equivalent to 154.8 cubic feet per second when it is expressed in terms of a uniform flow throughout the calendar year. The Normans kill Impoundment has a maximum usable capacity of less than 1,400 million qallons or less than 3.88 of the total annual runoff.

\section{Spillway Capacity and Overflow Potential of Existing Facilities}

Under the National Dam Safety Program, the Department of the Army, New York District, Corps of Engineers, required by letter dated March 31, 1978, a Phase I Inspection Report to be submitted evaluating the safety of the Watervliet Reservoir Dam. The report [Ref. 102] dated July 05, 1978 was prepared by Tippets-AbbettMcCarthy-Stratton and evaluated the flood potential of the site and the capacity of the facility to withstand such flooding. We will not duplicate the computations of this referenced report, but we will cite its stated conclusions as they pertain to evaluating the safety of the dam.

Based on a maximum possible head of water on the dam with the reservoir water surface at an elevation equal to the top of the end walls of the dam 8.5 feet above the spillway, and assuming a weir coefficient of 3.8 , the referenced report cites that the 324 foot long spillway has an overflow capacity, assuming no flashboards, of 30,500 cubic feet per second or 280 cubic feet per second per square mile of drainage area. 
The referenced report further points out that no continuous flow records were maintained for the Normans Kill prior to 1967, but that indirect discharge measurements taken after August-October 1955 floods indicated a maximum discharge of 13,300 cubic feet per second downstream at Slingerlands from a 169 square mile drainage area which the authors transposed to an equivalent value of 10,900 cubic feet per second or about 96 cubic feet per second per square mile at. the dam. At the U.S.G.S. gauging station established at Westmere in October 1967, the reported extreme maximum discharge for the 131 square mile watershed during a period of record through september 1977 is 5,580 cubic feet per second or about 50 cubic feet per. second per square mile.

The Dam Safety Inspection Report concludes its Hydraulic/Hydrologic discussion in section 5 with the following evaluation: "The spillway capacity without flashboards is 79 percent of the estimated Standard Project Flood and with about 1.5 feet flow over the abutments it would pass the entire flood: As the dam is located in a confined valley with rock abutments, overflow of the abutments will not cause significant erosion or undermine the foundation of the dam. Therefore, from a hydraulic and hydologic standpoint the spillway capacity is considered adequate."

\section{Flow Duration}

Traditionally, hydroelectric generating facility machine configurations and sizes are determined with a very heavy reliance on a graphical display of stream flow versus percent of time or probability that the stream flow will be less than that value. We feel that this approach is inapplicable here and is insensitive to the cost rate structure of the various alternative machine configurations, the discontinuous nature of the benefit rate structure, the individual usage requirements, marketing constraints and a large number of individual factors unique to this location, situation and owner. 
Initial Operational Model

Having created a data base of 50 years of monthly average runoff records, it was next necessary to determine how this data might be advantageously utilized. The study certainly would generate many different possible hydroelectric generating configurations, each with a variety of combinations of element sizes at different costs. The initial task would be to select the optimum combination of component elements to make a complete operating facility. The optimization process must be based primarily upon economic considerations, weighing the costs of various alternatives against their corresponding benefits. Costs consisting of initial capital costs and future costs in the form of labor and annual maintenance can be estimated with reasonable accuracy. The benefit side, or the return on investment, is the more difficult to assess since it requires a determination of the market value or credit accruable to the owner for the electrical energy which the facility as configured might be capable of producing. The economic benefits which result from the production of electrical energy cannot be determined knowing only the pertinent physical aspects of the various alternatives which will be investigated. It is first necessary to relate the quantification of the electrical energy production to the hydrologic factors, that is the availability of the natural resource, water.

The best method of investigating the performance of this type of an operating system is to mathematically model the intended operation and sequentially simulate the operation through each of the 600 months of synthetic data, making such simplifications as are necessary to enable computation to be made. We therefore developed a computer program to simulate the operation of a hydroelectric generating facility. The purpose of the program was to make a record of the length of time that the facility could operate and produce power for each month of the data period. It would be necessary to assess the effect that varying the volume of impoundment allocated to hydroelectric power generation would have on the calculated schedule of operation and power production. The 
initial model, therefore, was set up as a mass balance between inflows to the impoundment, drafts from it for water supply and hydroelectric power, and changes in volumetric storage of the impoundment, operating consistently under specified constraints.

The program permitted specification of the following variables:

1. The daily water supply requirement was assumed to be constant for each month of the computer run. A value of six million gallons daily was generally used and is a value which is unlikely to be exceeded in the near future.

2. The hydraulic flow rate to be used by the generating equipment was assumed to be held constant whenever the equipment is in operation.

3. The maximum permissible drawdown of reservoir level below the top of the flashboards is the level below which the turbine operation is required to cease. Water supply requirements, however, continue to be withdrawn from the reservoir regardless of. reservoir level. It is therefore possible to have storage deficits in excess of that specified during periods where inflows into the reservoir are less than the amount being withdrawn for water supply.

The following additional assumptions were incorporated into the initial operating model in order to facilitate computation:

1. Inflows into the impoundment for each month are assumed to be uniform through the monthly period. This assumption is idealized and results in the calculation of maximum running times and power production because short duration peak flows which might pass over the dam uncaptured when the reservoir is full may not be reflected. A modification to this assumption is dealt. with in a subsequent section.

2. Turbine operation is assumed idealisticaliy to be unlimited in its on-off cycling during periods where inflows are insufficient to run the turbines continuously without lowering reservoir storage below shutoff level. In actual operation, controls would be set up to establish a minimum differential starting and, stopping level, and some types of turbine equipment 
would be capable of operating at variable flow rates down to perhaps $10 \%$ of their maximum rates so that inflows in this low range could be captured for power production with minimum cycling. This computational assumption will therefore contribute little error in determining the equivalent running time of the turbine at its assumed flow rate.

3. Reservoir storage volume is assumed never to exceed the full reservoir level, nominally taken as elevation 259 at the top of the flashboards. Excess inflows into a full reservoir in any period are lost over the dam and are assumed to be not recoverable.

The program computes the number of days that the turbine is capable of operating at its selected flow rate without drawing reservoir storage below the specified level during each month using the simulated inflows. The residual reservoir storage volume is calculated at the end of each month and is then used as the starting point for the following month's operation. The number of operating days for the following month is then calculated using the new starting reservoir volume and the next sequential monthly mean inflow rate from the flow simulation. The computer output provides a 50 year summary stating the three variables specified and listing the number of operating days at full capacity for each month of the 50 year sequence, the total number of operating days for each of the 50 annual sequences, the minimum and maximum month end reservoir storage deficit below the full reservoir level expressed in millions of qallons for each 12 month sequence, and the average number of operating days for each month and each year of the 50 year sequence.

A large number of program runs (simulations) were made to gain information over a range of turbine capacities from zero to 800 cubic feet per second and for various specified maximum drawdown levels. Based on these results, we have plotted as Figure 24 the relationship between the average annual number of days a turbine comfiguration can operate at full capacity, the turbine flow rate, and the maximum amount of reservoir level drawdown permitted for turbine operation. It must be remembered that this graph presents 
an arithmetic average condition over a fifty year simulation period and that extreme variations in operating time exist from one year to the next. This graph does not tell us anything about the distribution of operating days during the year. As anticipated, the graph shows us that a greater number of operating days can be achieved annually by reducing the turbine size and by increasing the amount of storage which can be allocated to turbine operation.

\section{Refinements To The Operational Model}

Reliable stream flow data is available only on a monthly average basis. Our initial operational model made an assumption that daily inflows in any month were uniform throughout that month. This was admittedly an inaccurate assumption and we acknowledge that the turbine operating times and power produced are maximized under such an assumption as demonstrated by the following hypothetical example.

Suppose for example, that the mean monthly flow for a sample 30 day month was approximately equal to the turbine capacity of say 200 cfs, but that the inflows actually occurred in such a way that 608 of the runoff for that month occurred uniformly during the first six days of the month while the remaining 408 of the monthly inflows occurred uniformly during the remaining 24 days of the month. Assuming that the inflows were uniform during the month, we would conclude that the turbine would run 30 days during the month. Based on the actual inflow distribution, however, we would conclude that the reservoir level would begin to fall after the sixth day, dropping the level in the reservoir approximately three feet in approximately a five day period causing the turbines to cycle near the maximum drawdown level while running approximately $50 \%$ of the remaining time in the month. In the actual case, the turbines would have operated approximately 20 days for that month or only two thirds as much time as that determined assuming uniform inflows. If high flows occur, however, while the reservoir level is low, they will to some degree, be captured to replenish previously depleted storage. 
The uniform inflow assumption used in our initial operational model needed to be modified to make the results more closely reflect non-uniform rates of inflow during any month. We chose to modify the simulation model in such a way that the monthly computation of turbine running time would be based upon the same total inflow for the month but the inflow would be assumed to occur during two periods, one as a uniform high flow period and the second as a uniform low flow period. Analysis of the frequency distribution curves of mean inflows for each month pointed out for instance that for October approximately two-thirds of the total reservoir inflow could be accounted for by only $20 \%$ of the months. In contrast, approximately one-third of the total inflows for the month of March were accounted for by the highest 208 of the values. It seemed somewhat reasonable, therefore, that a. realistic distribution of flows within a given month might in some way attempt to maintain the same relationship in the daily distribution for our operational model. We were able to approximate this relationship by using the following method.

Each month was assumed to have a base inflow equal in magnitude to the 20 percentile flow which can be determined using Figure 23. The high flow for the month is assumed to occur during the first $20 \%$ of time in the month. The magnitude of the flow during the high flow period was taken to be equal to the base flow plus three times the amount that the monthly average flow exceeds the base flow. Flow during the remaining $80 \%$ of the month was taken to be equal to the base flow plus one-half the amount by which the monthly mean flow exceeds the base flow. Flow during each of these two portions of the month is assumed to be uniform at those rates, with 608 of the month's excess over the base flow situation assumed to be uniformly distributed during the first 208 of the month. We feel that this modification to the operational model is reasonable, is an improvement over the uniform inflow assumption, and is necessarily simple to be able to carry out the computation. 
Many program runs were made using the modified operational model over the same range of input variables as were used with the initial operational model. We have taken the results determined by simulated operation with the modified operational model as being representative of the running times which might be reasonably expected over a 50 year period for various power generating configurations. We have plotted in Figure 25 the relationship between the average number of days a turbine configuration can be operated at full capacity, the turbine flow rate, and the maximum amount of: reservoir level drawdown permitted for turbine operation. As predicted, this graph shows a reduction in operating days from Figure 24 because of the modified inflow assumption. The reduction, however, is not as dramatic as one might have expected.

Figure 26 includes typical simulation output which display the 50 year monthly summaries of simulated turbine operations at 50 , $100,200,300,400,500,600$ and 800 cfs turbine capacity predicated on a maximum reservoir drawdown level of three feet, and maintenance of a domestic water supply of 6 million gallons daily. The results displayed on these sheets form a basis for the later determination of economic benefits for the many alternative configurations.

\section{Conclusions}

We have determined that operation with an increased permissible reservoir drawdown will result in lengthier operating times. We must now determine whether the increased operating times will be beneficial to the operation, or whether it is safe from a water supply point of view or desirable from an environmental or recreational point of view to operate the reservoir at levels siqnificantly below the full level.

The amount of power that can be generated by any given turbine confiquration can be calculated by the expression:

$$
\text { Power }=\text { Head } \times \text { filow } \times \text { Erficiency } \times 0.08461
$$

where head is the net head in feet to be utilized by the turbine, flow is expressed in cubic feet per second, and efficiency is expressed as a decimal. The various configurations to be 
investigated will all have their own unique set of each of these parameters. It is desirable at this point to make some power computations requiring the application of some uniform assumptions. Let us assume that there is a net head of 60 feet available which is a similar value for many of the alternative configurations. Further assuming that a typical efficiency value for most of the configurations is $80 \%$, we can therefore express the total power output from our hypothetical generating facility knowing the amount of time that the machine will operate and the flow rate utilized by the turbine. We have prepared Figure 27 which graphically shows the power which might be developed by our hypothetical turbine based upon the average number of operating days from our 50-year simulations and various turbine capacities. The graph demonstrates the fact that the maximum amount of power is generated under the uniform monthly inflow assumption made in our initial operational model.

In order to be able to make computations, it was necessary to make an assumption regarding the average head of water available on the turbines under conditions of operation where increased reservoir drawdowns are permitted. Inspection of the many simulations has indicated that the reservoir would be operated at least two-thirds of its operating time at or near the maximum drawdown level. For purposes of this computation, therefore, we have made an assumption that the average drawdown during any of the simulations will be two-thirds of the allowable drawdown for turbine operation. Assuming that 60 feet of net head is available to the turbine when the maximum drawdown available is specified as 3 feet, we have graphed the average annual power available versus hydraulic turbine capacity. The average number of operating days in that computation is taken from from the computer printouts of Figure 26. It can be seen that less power is actually available when an increased amount of drawdown is permitted because the lowering of water level in the reservoir reduces the net head available on the turbine by an amount which more than compensates for the increased turbine running time. Therefore, we find that there is no advantage or necessity in the 
production of power to permit extremely large drawdowns in the reservoir. We would conclude that is undoubtedly desirable to allocate up to 3 feet of drawdown to be available for generation of hydroelectric power so that storage is available for short-term variations in daily flows but that additional drawdowns are unwarranted. This conclusion allows the establishment of future operating rules so that water supply and recreation will not be adversely affected while maximum benefit is being taken for hydroelectric generation. With regard to water supply, allocating approximately 3 feet or 350 million gallons from the top of the reservoir for hydroelectric production might cause the safe yield of the reservoir for water supply at a 99 percent reliability based upon maximum deficit of 1,200 million gallons to be reduced from 12 mgd to $10 \mathrm{mgd}$. This relationship is determined from Figure 8 and discussed in Segment one.

Using the same assumption that 60 feet of net head would be available for turbines operating at 808 efficiency and limited by a maximum reservoir drawdown of 3 feet, we prepared Figure 28 which is a graph showing the average amount of power which might be able to be produced for a wide range of hydraulic turbine capacities. We have attempted to impart continuity to the graph using a method similar to that described previously for Figure 23. The set of curves are useful to help us visualize the relationship between power produced in various times of the year and machine size in terms of flow rate. It is interesting to note the larger machine sizes can produce no more power than the smaller machine sizes during periods of low runoff when the smaller machine is capable of utilizing all the available inflows. The larger machine configurations are advantageous particularly during the spring months when high flows normally occur and would otherwise be lost over the dam by the smaller machines. The limiting power production value indicated on Figure 28 for the 50, 100, and 200 cfs machines during March and April show that machines of those sizes can be expected to operate continuously at peak capacity during that period in most years. For the sake of comparison, we have also 
superimposed on Figure 28 a line which represents the amount of electrical energy consumed by facilities owned by the City of Watervilet during the corresponding calendar periods of a calculated typical year. We have also indicated on Figure 28 the averaqe daily power which could theoretically be generated if all water passing over the dam could be utilized. All power production computations for Figure 28 are uniformly based on the 50 year. reservoir simulation and the same head and efficiency assumptions. This Figure conveys considerable visual information comparing machine configuration sizes with the power requirements of the city and the ultimate capability based on available water.

Using the same power generating parameters that were used in Figure 28, Figure 29 summarizes on an averaqe annual basis for the 50 year simulation period the total electrical power in megawatt hours which could be generated in turbine configurations having a hydraulic capacity from 0 to 800 cubic feet per second. The maximum amount of power which could be generated utilizing all flows over the dam regardless of the magnitude of those flows is shown as a solid line across the top of the graph at a value of 5,177 megawatt hours. The figure shows relatively larqe incremental gains in the amount of power that can be produced by an increase in turbine capacity at the low end of the turbine capacity scale, while reflecting very small incremental gains by corresponding increases in turbine capacity at the higher end of the turbine capacity scale.

Having determined that reservoir levels need not be significantly depressed for optimum hydroelectric power generation, it is increasingly obvious that the dual usages of the Normans Rill Impoundment site for both municipal water supply and hydroelectric power generation are compatible. It was previously mentioned that the usable impoundment volume was less than 3.88 of the total annual watershed runoff. This proportion is very small and effectively limits the uniform reliable water supply yield of the reservoir to less than 128 of the mean annual flow leaving a very large quantity 
of water which cannot be impounded and which otherwise escapes unused from this site dissipating its energy in its passage over the dam to the streambed below.

The generation of hydroelectric power at this site will divert water through turbines which would overflow the dam converting the otherwise wasted energy of this flow into beneficial electrical energy. 


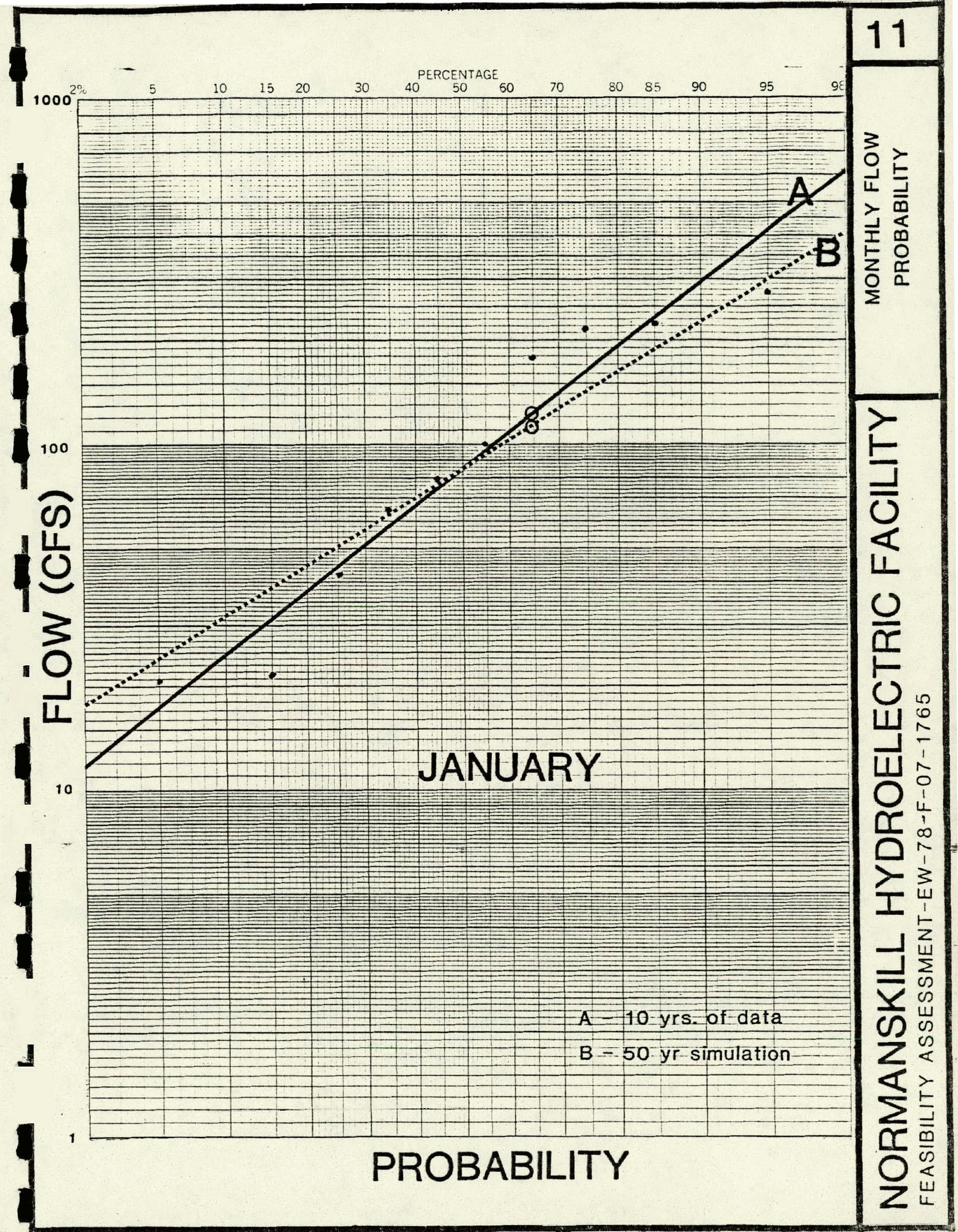




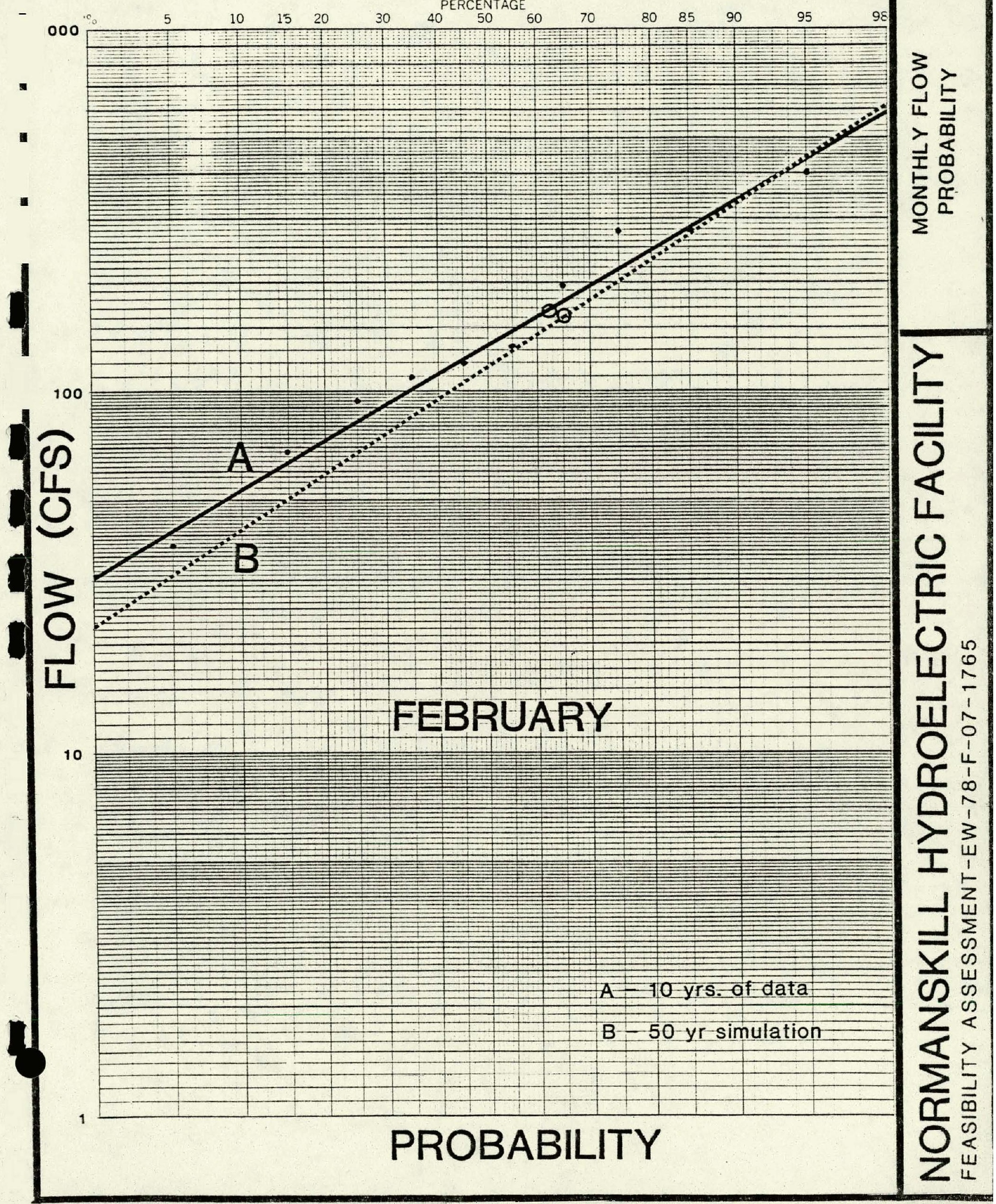




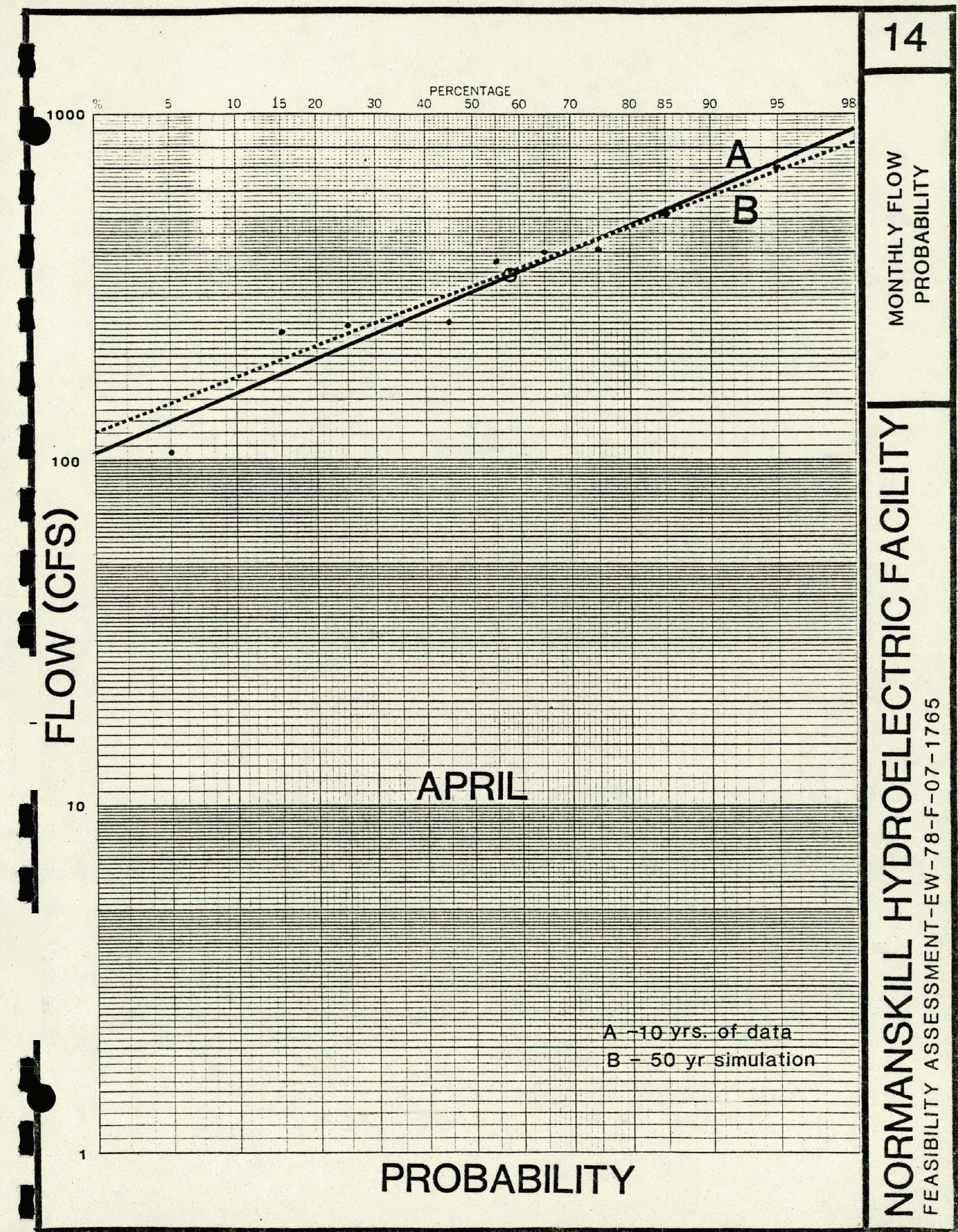




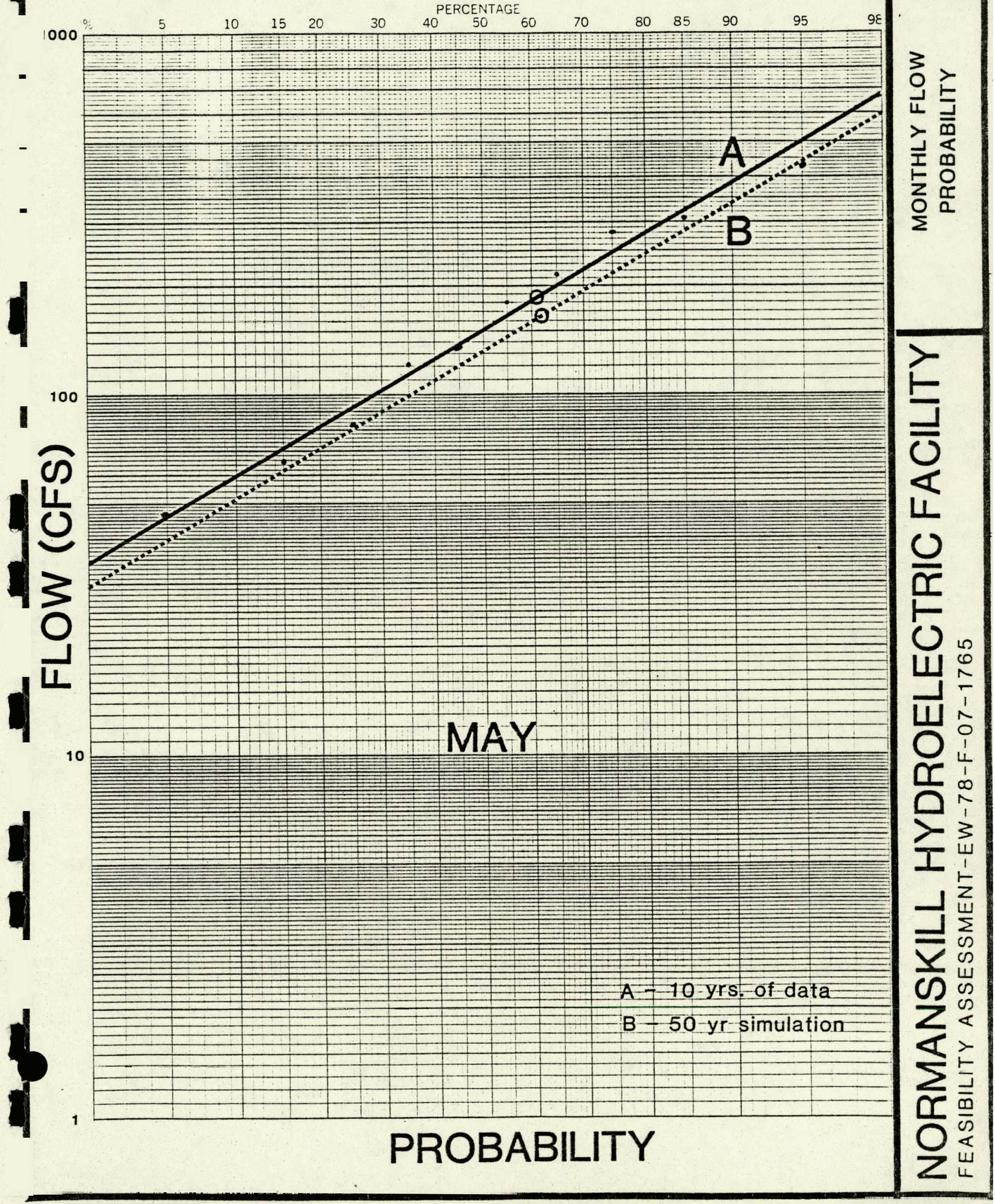



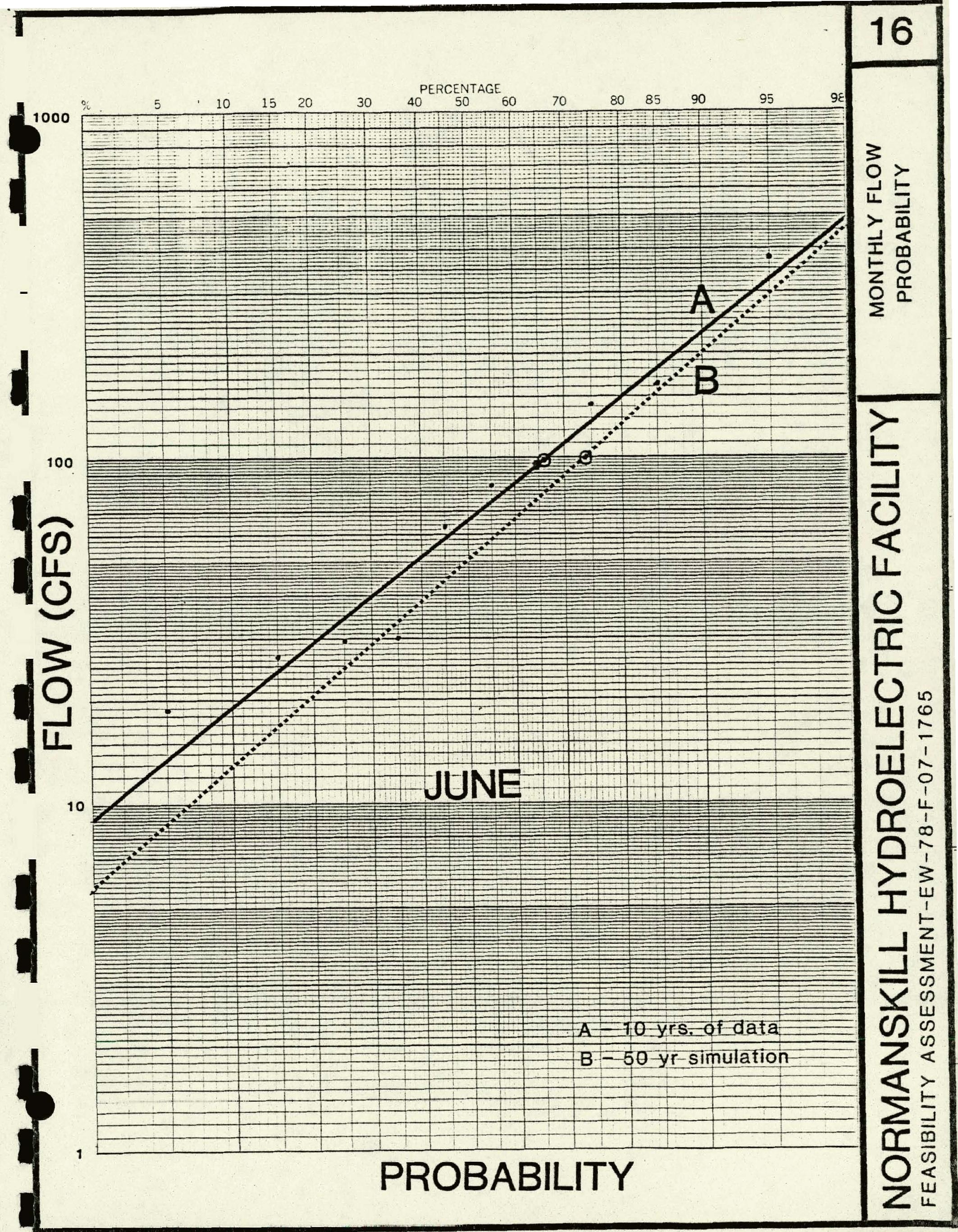


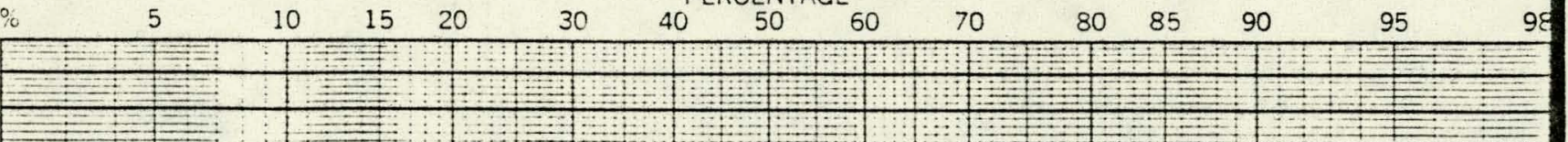




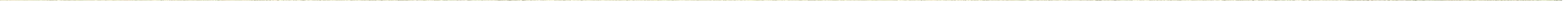


$10 \quad 15 \quad 20 \quad 30 \quad 40$ (
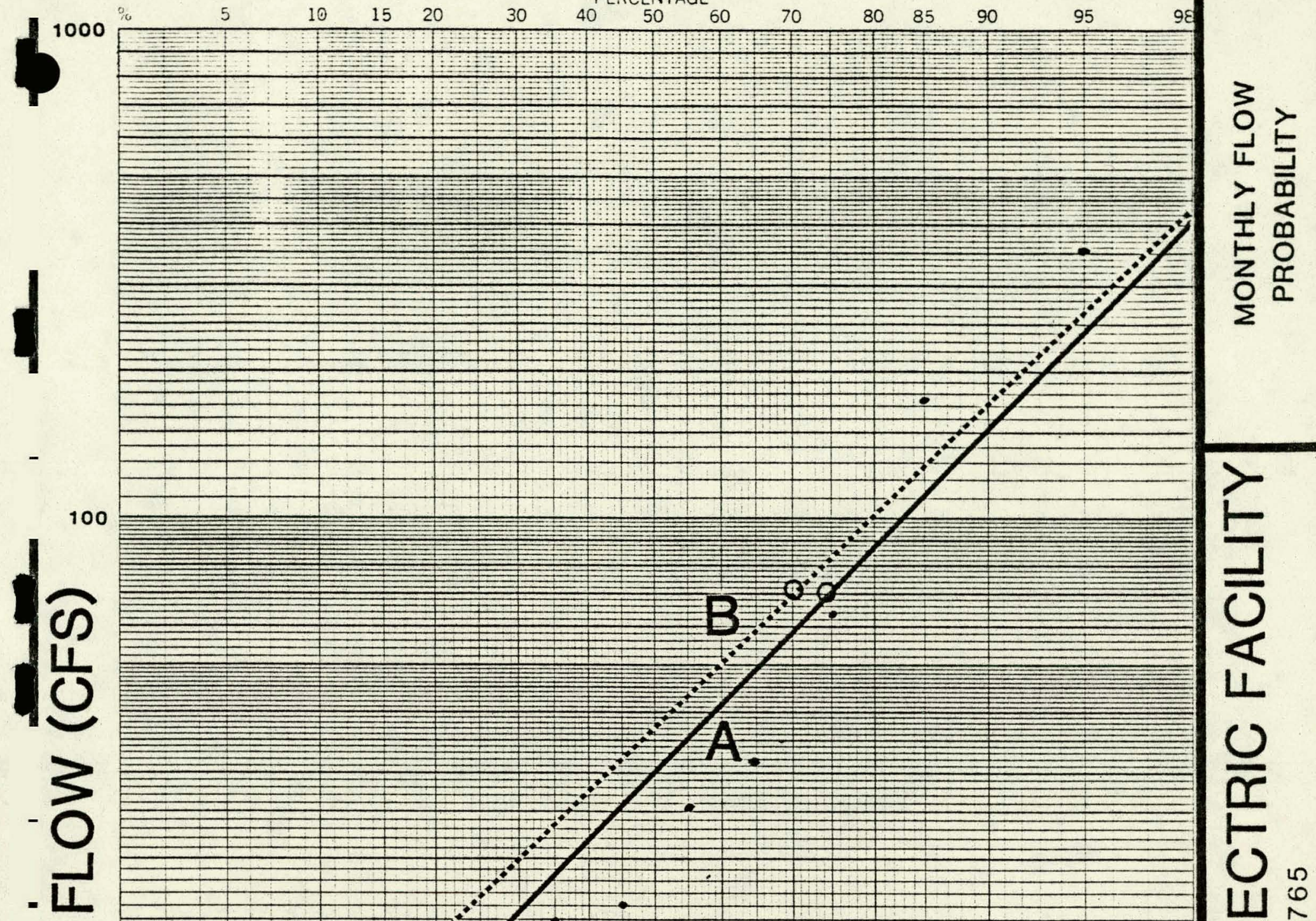

10

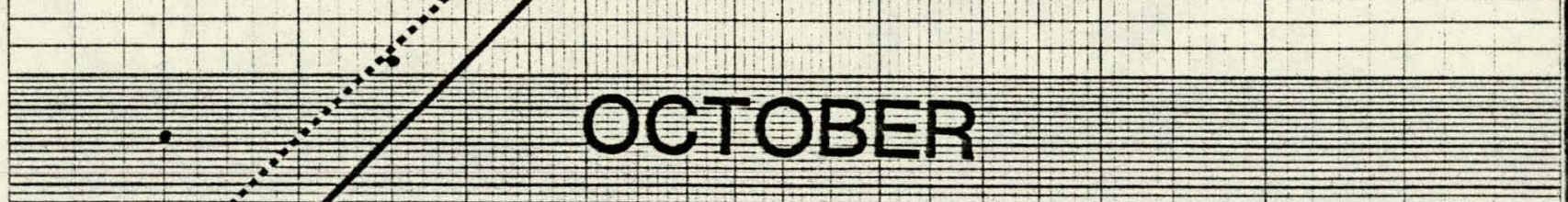

OCTOBER

A - 10 yrs. of data $B-50$ yr simulation 


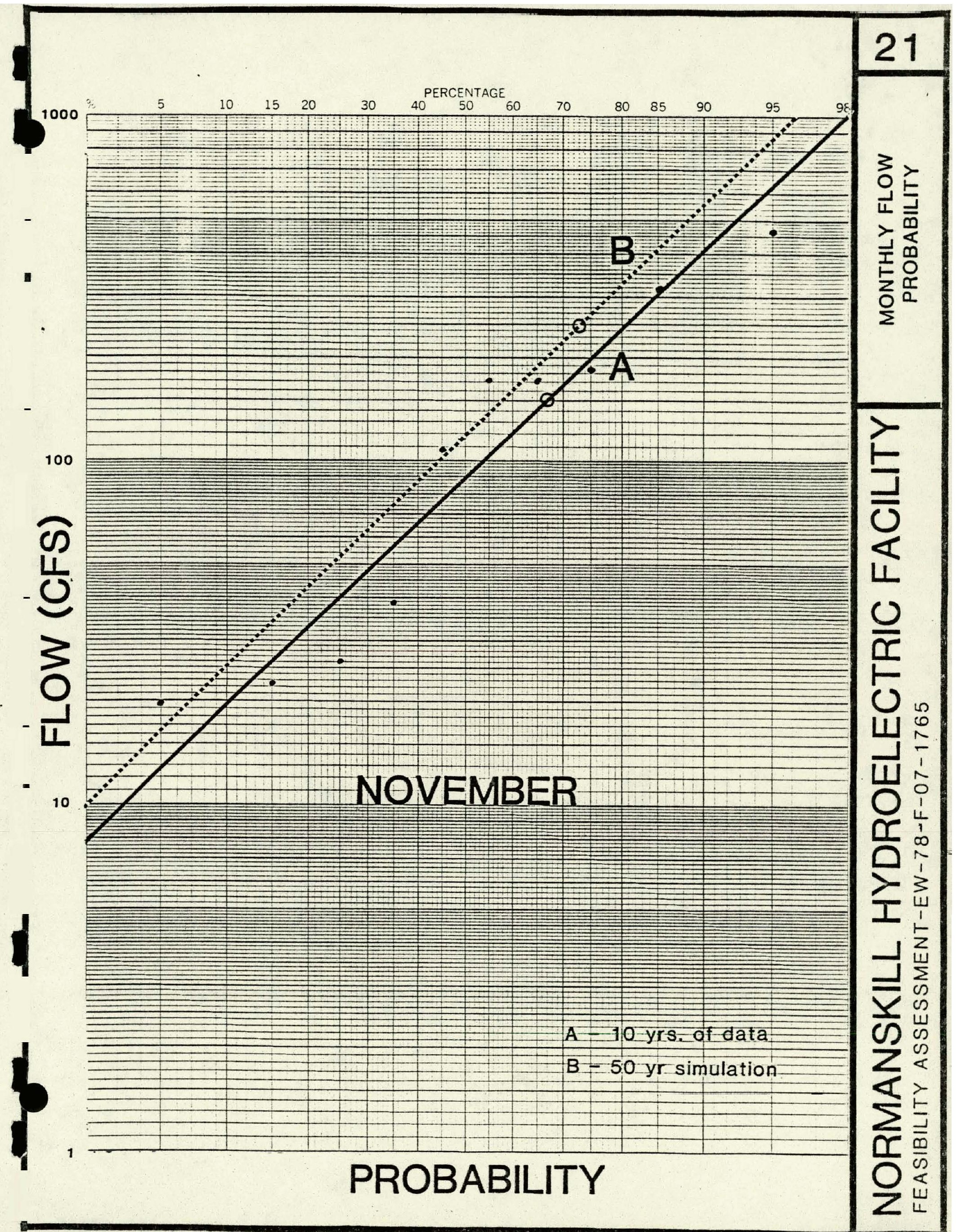




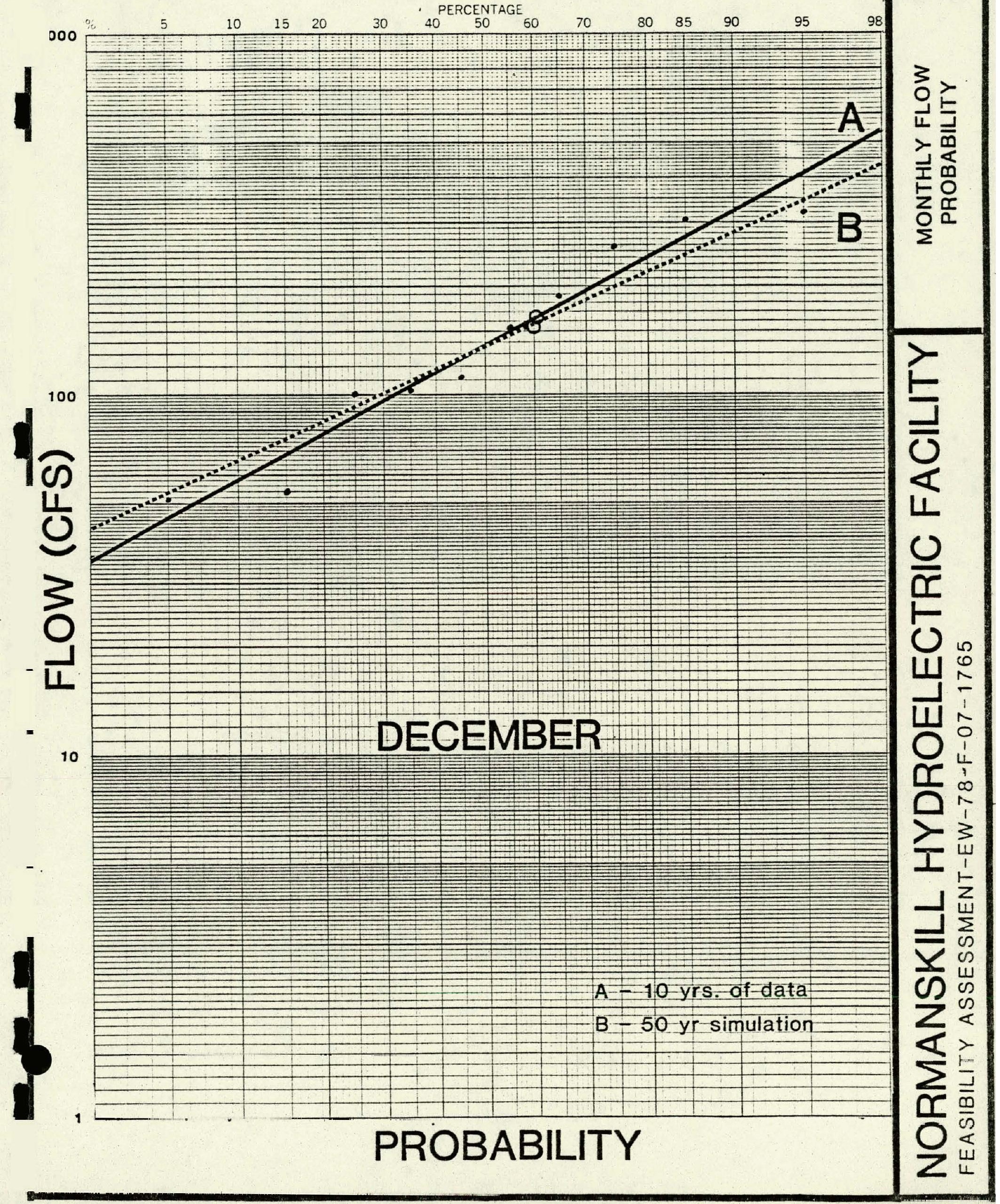




\section{MONTHLY MEAN FLOW PROBABILITY}

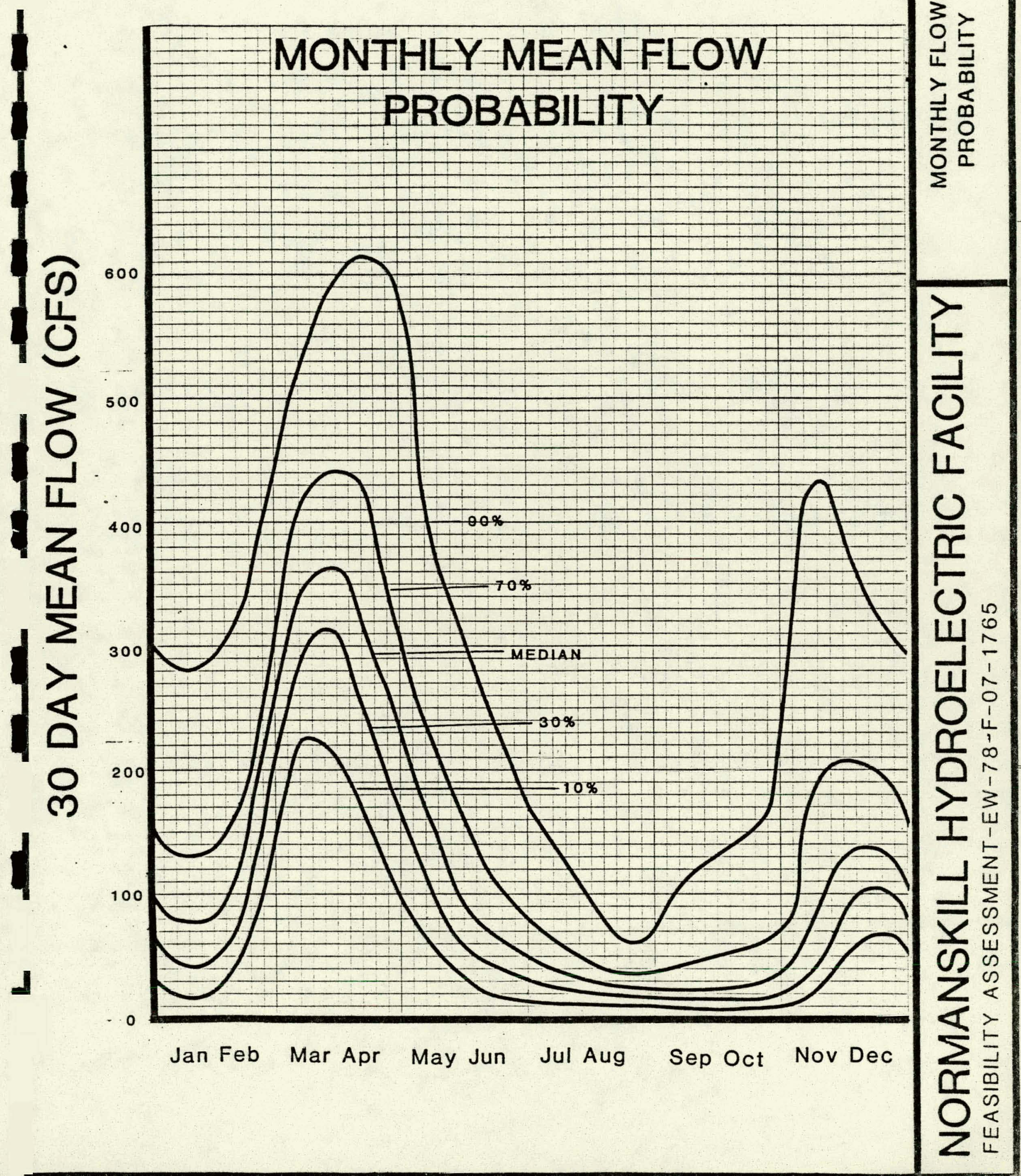

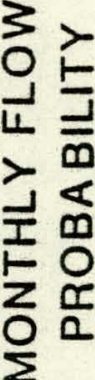





\section{TYPICAL
RESERVOIR SIMULATION OUTPUT \\ TYPICAL
RESERVOIR SIMULATION OUTPUT}

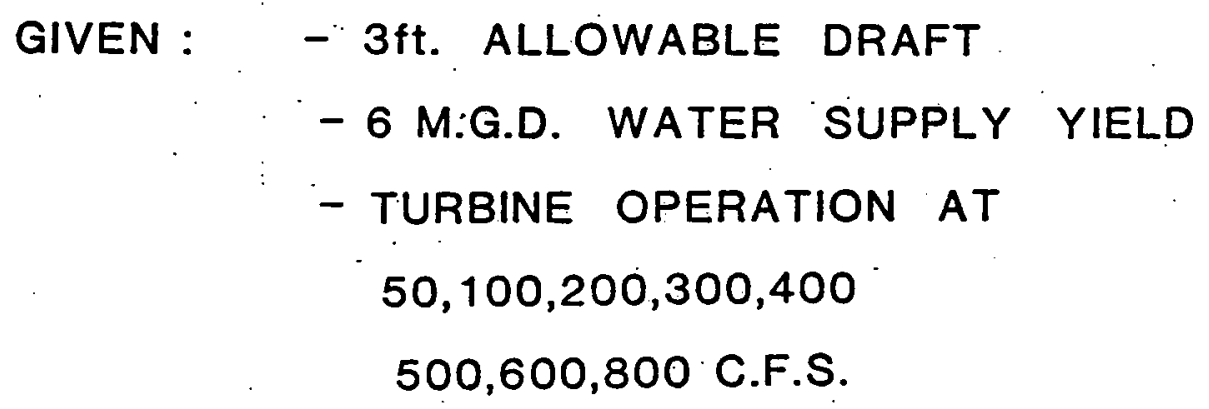


WATERVLIET FESERVOIR HYIRO-ELECTPIC STUIY EASEI ON STREAMFLDW SYIVIHESLS

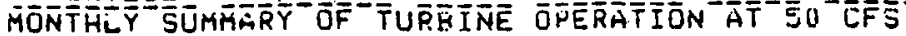

ASSUHING: OPERATION CEASES AT 3 FEET BELOW FLASHEOARIS

DOMESTIC WATER SUPPLY MAINTAINEI AT S MGD

DEFICIT IMO

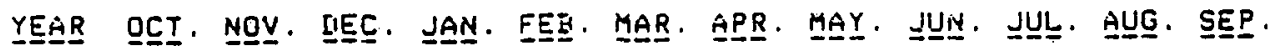

IOTAL

MIN. MAX.

$\begin{array}{llllllllllllllll}1 & 13.6 & 30.0 & 25.5 & 13.9 & 17.7 & 31.0 & 30.0 & 31.0 & 30.0 & 20.9 & 4.2 & 8.9 & 250.7\end{array}$

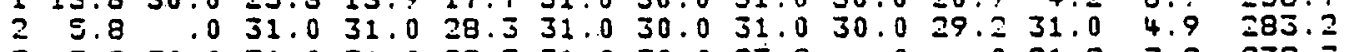

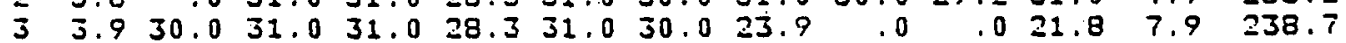

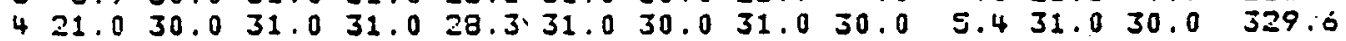

$531.0 \quad 30.0 \quad 31.0 \quad 31.0 \quad 28.3 \quad 31.0 \quad 30.0 \quad 31.0 \quad 30.0 \quad 31.0 \quad 28.3 \quad 30.0 \quad 362.5$

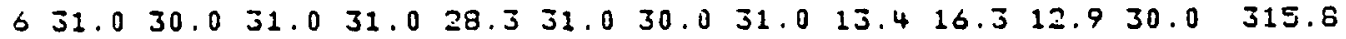

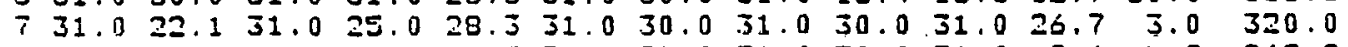

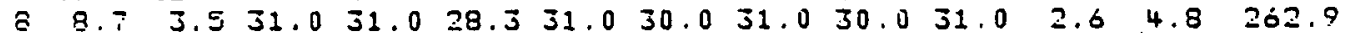

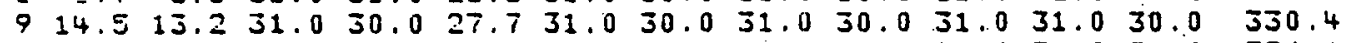

$\begin{array}{lllllllllllll}10 & 21.9 & 0 & 31.0 & 31.0 & 28.3 & 31.0 & 30.0 & 31.0 & 30.0 & 31.0 & 31.0 & 30.0\end{array}$

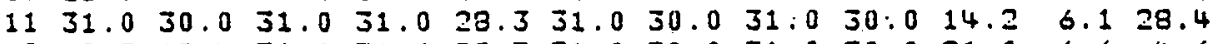

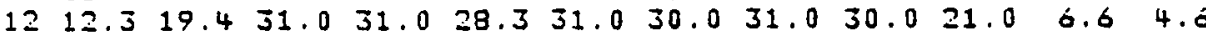

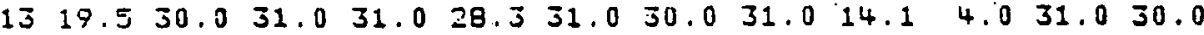

$1431.0 \quad 30.0 \quad 31.0 \quad 31.0 \quad 7.831 .0 \quad 30.0 \quad 31.0 \quad 30.0 \quad 31.0 \quad 31.0 \quad 30.0$

$\begin{array}{lllllllllllll}15 & 31.0 & 26.1 & 25.7 & 25.2 & 28.3 & 31.0 & 30.0 & 31.0 & 28.5 & 25.7 & 15.3 & .0\end{array}$

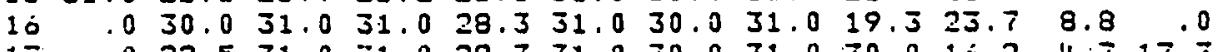

$17.022 .531 .0 \quad 31.0 \quad 28.3 \quad 31.0 \quad 30.0 \quad 31.0 \quad 30.0 \quad 10.2 \quad 4.7 \quad 17.3$

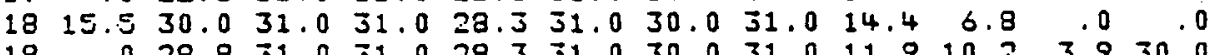

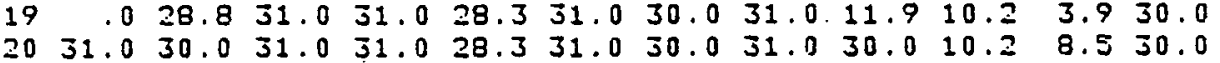

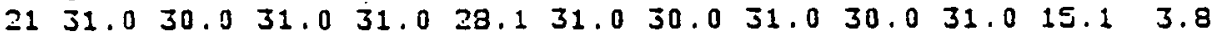

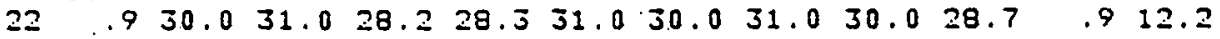

$2310.5 \quad 30.0 \quad 31.0 \quad 17.3 \quad 28.3 \quad 31.0 \quad 30.0 \quad 25.8 \quad 7.3 \quad 13.7 \quad 10.110 .3$

$\begin{array}{lllllllllllll}24 & 16.0 & 30.0 & 31.0 & 31.0 & 28.3 & 31.0 & 30.0 & 31.0 & 23.5 & 13.3 & 4.4 & 2.6\end{array}$

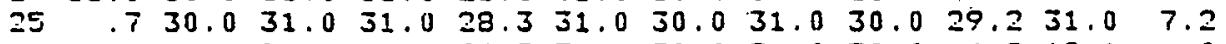

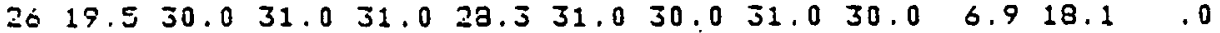

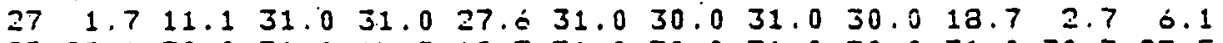

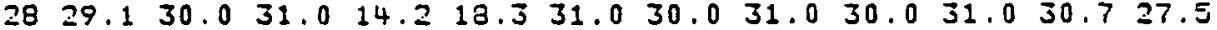

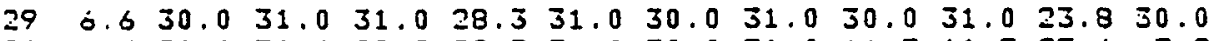

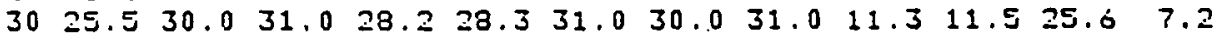

$\begin{array}{lllllllllllll}31 & 9.0 & 30.0 & 31.0 & 29.5 & 10.0 & 31.0 & 30.0 & 31.0 & 29.2 & 31.0 & 4.0 & .0\end{array}$

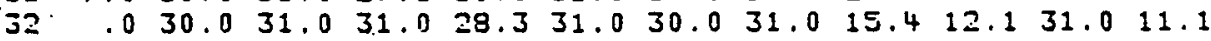

$\begin{array}{lllllllllllll}33 & 3.1 & 30.0 & 31.0 & 31.0 & 28.3 & 31.0 & 30.0 & 31.0 & 30.0 & 31.0 & 23.5 & 30.0\end{array}$

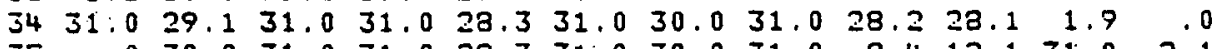

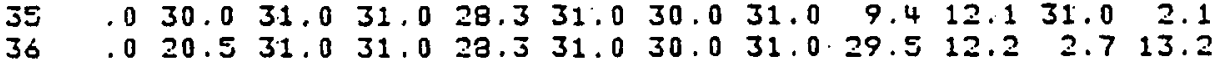

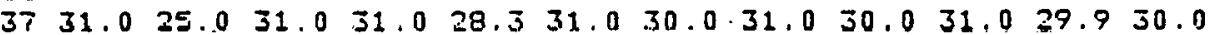

$\begin{array}{lllllllllllllll}38 & 31.0 & 28.4 & 31: 0 & 31.0 & 28.3 & 31.0 & 30.0 & 31.0 & 30.0 & 11.0 & 8.0 & 3.3\end{array}$

$39 \begin{array}{lllllllllllll}2 & 12.8 & 30.0 & 31.0 & 24.7 & 28.3 & 31.0 & 30.0 & 23.1 & .0 & .4 & 1.1 & 14.2\end{array}$

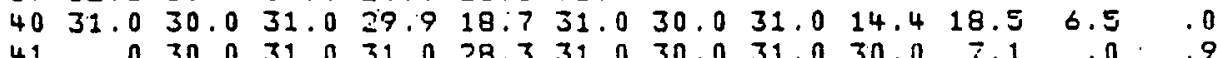

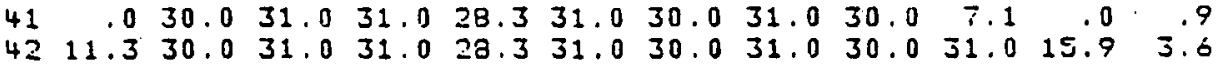

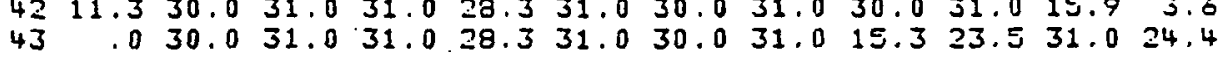

$\begin{array}{llllllllllllll}44 & 31.0 & 30.0 & 31.0 & 31.0 & 28.3 & 31.0 & 30.0 & 31.0 & 30.0 & 31.0 & 24.5 & 30.0\end{array}$

$4531.0 \quad 30.031 .0 \quad 31.0 \quad 28.3 \quad 31.0 \quad 30.0 .31: 0 \quad 21.215 .431 .030 .0$

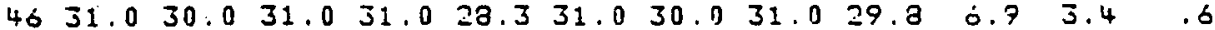

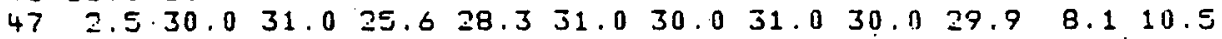

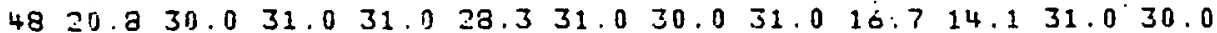

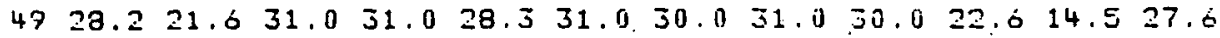

$5011.730 .0 \quad 31.0 \quad 31.0 \quad 21.2 \quad 31.0 \quad 30.031 .030 .031 .0 \quad 26.0 \quad 10.5$

326.1

322.0

276.1

311.0

344.8

297.7

264.0

273.0

249.0

267.1

322. 0

322.9

282.2

251.2

272.1

310.3

286.7

251.9

333.8

333.6

290.5

265.8

281.9

329.7

300.6

260.9

260.3

359.2

293.7

226. 5

272.0

250.2

304.0

306.5

358.7

340.8

283.7

287.9

324.9

326.9

314.9

$0 \quad 350$

$0 \quad 395$

$0 \quad 385$

$\begin{array}{ll}0 & 350 \\ 0 & 350\end{array}$

0350

00350

$0 \quad 350$

0 379

0 350

- 350

350

350

350

527

598

430

628

350

350

350

350

350

350

350

409

350

350

356

443

537

350

350

463

374

350

350

$380^{\circ}$

350

464

350

उ०3

350

350

350

350

$35 \hat{~}$

35:

AVG. $16.420 .430 .829 .426 .731 .0 \quad 30.930 .024 .520 .1 \quad 16.514 .0$

ว96. 7

o.26 


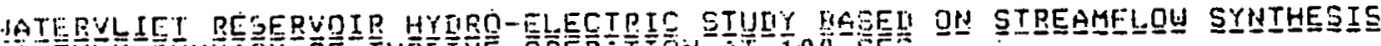

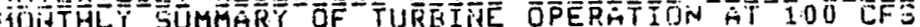

GSSUMING: OPERATION CEASES AT 3 FEET BELOW FLASHEDARUS

DOMESTIC HATER SUPPLY MAINTAINEI AT O MGI

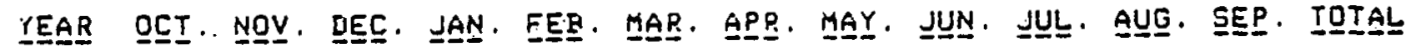

DEFICIT SMG:

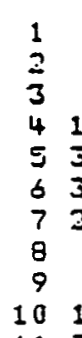

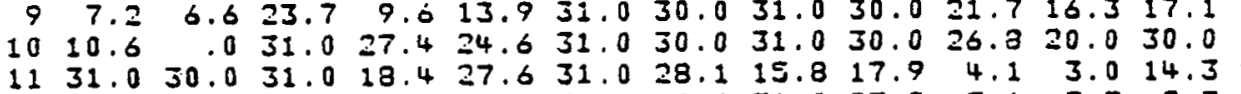

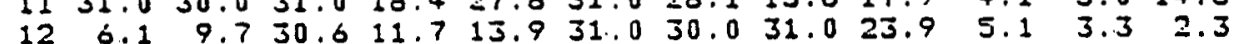

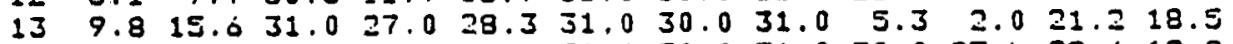

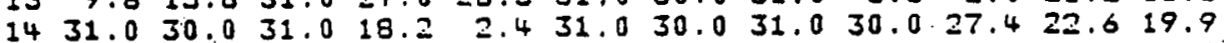$$
1520.810 .812 .812 .626 .131 .030 .027 .98 .812 .97 .7
$$$$
15
$$$$
16
$$$$
17
$$$$
\begin{aligned}
& 19 \\
& 20
\end{aligned}
$$$$
20
$$$$
2
$$

$$
22
$$

24

2

25

27

28

2

$\begin{array}{llllllllllllll}31 & 4.5 & 30.0 & 31.0 & 14.8 & 5.0 & 31.0 & 30.0 & 31.0 & 14.7 & 20.6 & .0 & .0\end{array}$

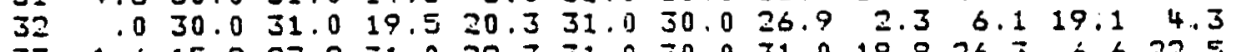

$\begin{array}{lllllllllllllll}3 & 1.0 & 15.8 & 27.8 & 31.0 & 28.3 & 31.0 & 30.0 & 31.0 & 19.8 & 26.3 & 6.6 & 22.5\end{array}$

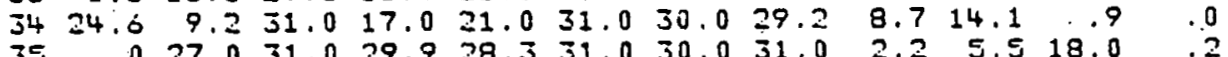

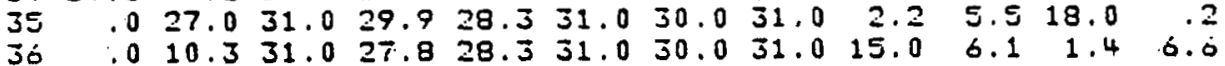

3720.111 .031 .026 .527 .631 .030 .026 .510 .425 .714 .623 .9

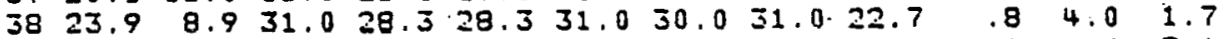

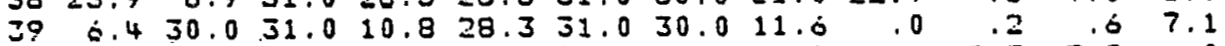

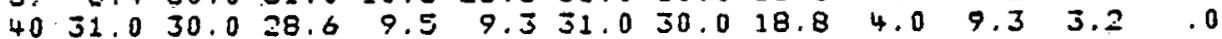

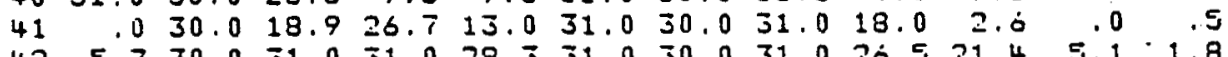

$\begin{array}{rrrrrrrrrrrrrr}42 & 5.7 & 30.0 & 31.0 & 31.0 & 28.3 & 31.0 & 30.0 & 31.0 & 20.5 & 21.4 & 5.1 & 1.8 \\ 43 & 0 & 23.6 & 31.0 & 31.0 & 28.3 & 31.0 & 30.0 & 31.0 & 7.5 & 11.8 & 22.2 & 9.3\end{array}$

$\begin{array}{rrrrrrrrrrrrr}43 & .0 & 23.6 & 31.0 & 31.0 & 28.3 & 31.0 & 30.0 & 31.0 & 7.5 & 11.8 & 22.2 & 9.3 \\ 44 & 30.1 & 30.0 & 31.0 & 31.0 & 28.3 & 31.0 & 30.0 & 31.0 & 30.0 & 31.0 & 8.2 & 30.0\end{array}$

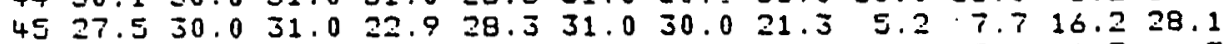

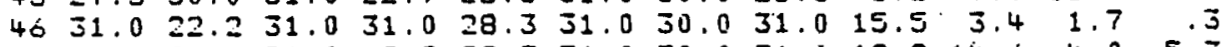

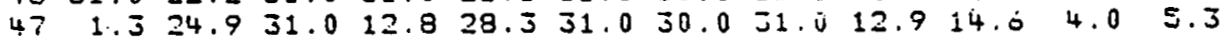

4810.430 .0 .31 .0 23.7 $28.3 \quad 31.0 \quad 30.019 .0 \quad 4.8$ 7.0.10.1 18.5

$4913.510 .8 \quad 31.028 .428 .3 \quad 31.0 \quad 30.031 .0 \quad 29.9 \quad 5.9 \quad 7.313 .8$

50 5.8 $30.0 \quad 31.021 .4 \quad 7.8 \quad 31.0 \quad 30.031 .0 \quad 30.023 .110 .4 \quad 5.2$

AVG. $11.721 .8 \quad 28.521 .8 \quad 22.8 \quad 31.0 \quad 30.0 \quad 27.0 \quad 15.8 \quad 12.2 \quad 3.4 \quad 9.3$
190.8

24.7 .8

206.3

260.2

311.0

249.7

241.9

207.7

238.1

292.4

252. 2

19.8 .6

250.7

304.4

201.5

228. 0

226.1

225.0

188.4

267.8

266.5

222.6

185.3

242.2

265.1

$220 . ?$

191.4

256.9

276.3

234.4

212.6

220.5

271.6

216.6

234.0

218.3

278.3

241.4

186.9

204.7

201.7

27.2 .8

256.6

341.6

279.1

256.3

226.9

249.8

260.9

250. 8

240.8
350

395

385

350

350

$35 i$

350

350

350

$37 ?$

350

350

350

350

350

527

598

430

628

उ5:

350

350

350

350

350

350

409

350

350

350

467

$56 \mathrm{i}$

350

350

463

374

350

350

386

356

$4 \therefore 4$

350

303

350

350

550

350

350

350

उ5

628 
IATERVLICT RESERVOIP HYIRO-ELECTRIE STULY HASEI ON STREAMFLOU ZIRTIHESIS RUTHTHLY SUTH

ASSLHIHG: OPERATION CEGSES AT 3 FEET EELOW FLASHHOARIS

[IOHESTIC WATER SUPPLY MAINTAINEII AT O MGLI

IIEF ICIT :MG

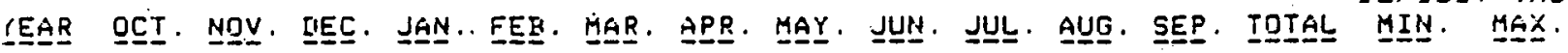

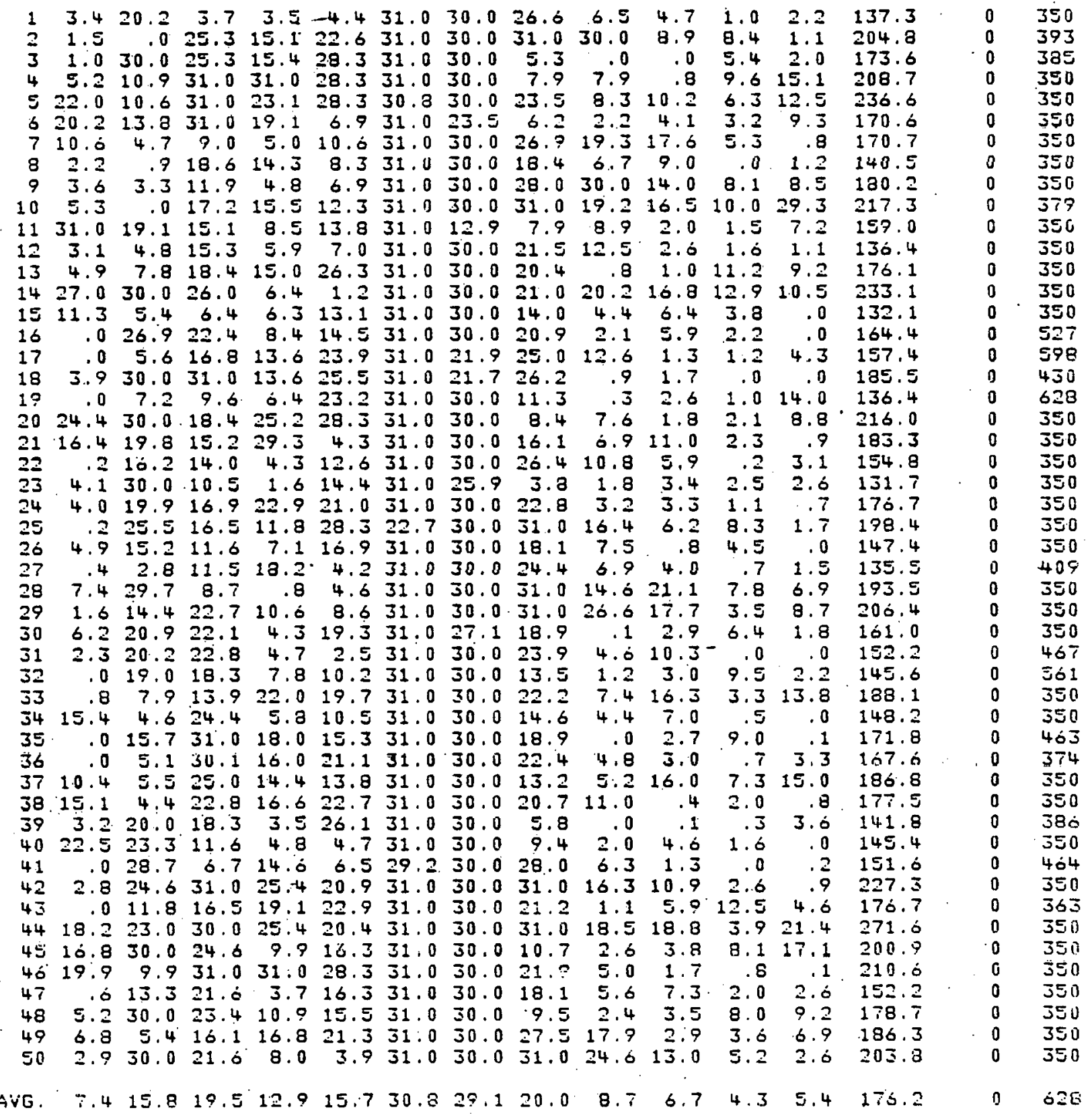


INTERYL IET RESERVOIR HYIRO-ELECTPIC STUIT GASEI ON STREAMFLDW SINTHESIS जOOH THL

ASSUMING: OPERATION CEASES AT 3 FEET BELOW FLASHEOARDS

DOMESTIC WATER SUPPLY MAINTAINEI AT O MGD

DEFICIT

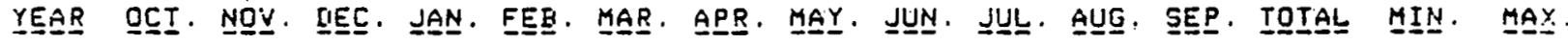

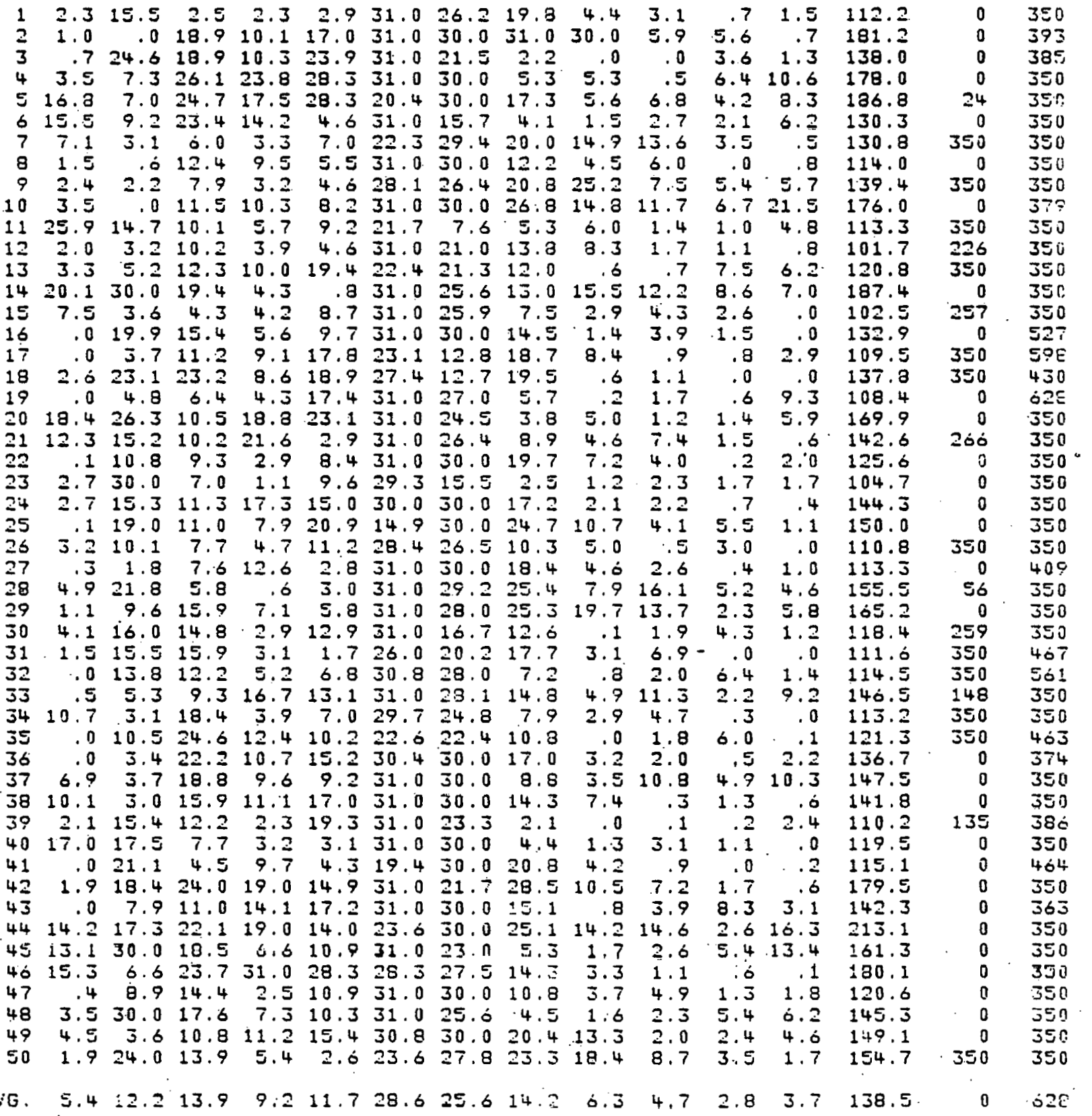


JATERVLIET RESERVOIR HYRPO-ELECTRIC STUTYY EASED ON SIREAMFLOW SYYNTHESIS IONTHLY SUMTÁPY OF TURE INE OPERATTION ATT 400 CFF

AESUMING: OPERATION CEASES AT 3 FEET BELOW FLASHBOARDS

DOMESTIC WATER SUPPLY MAIHTAINED AT 6. MGI

UIEFICIT (MG:

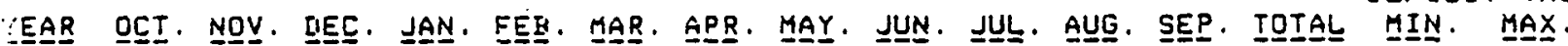

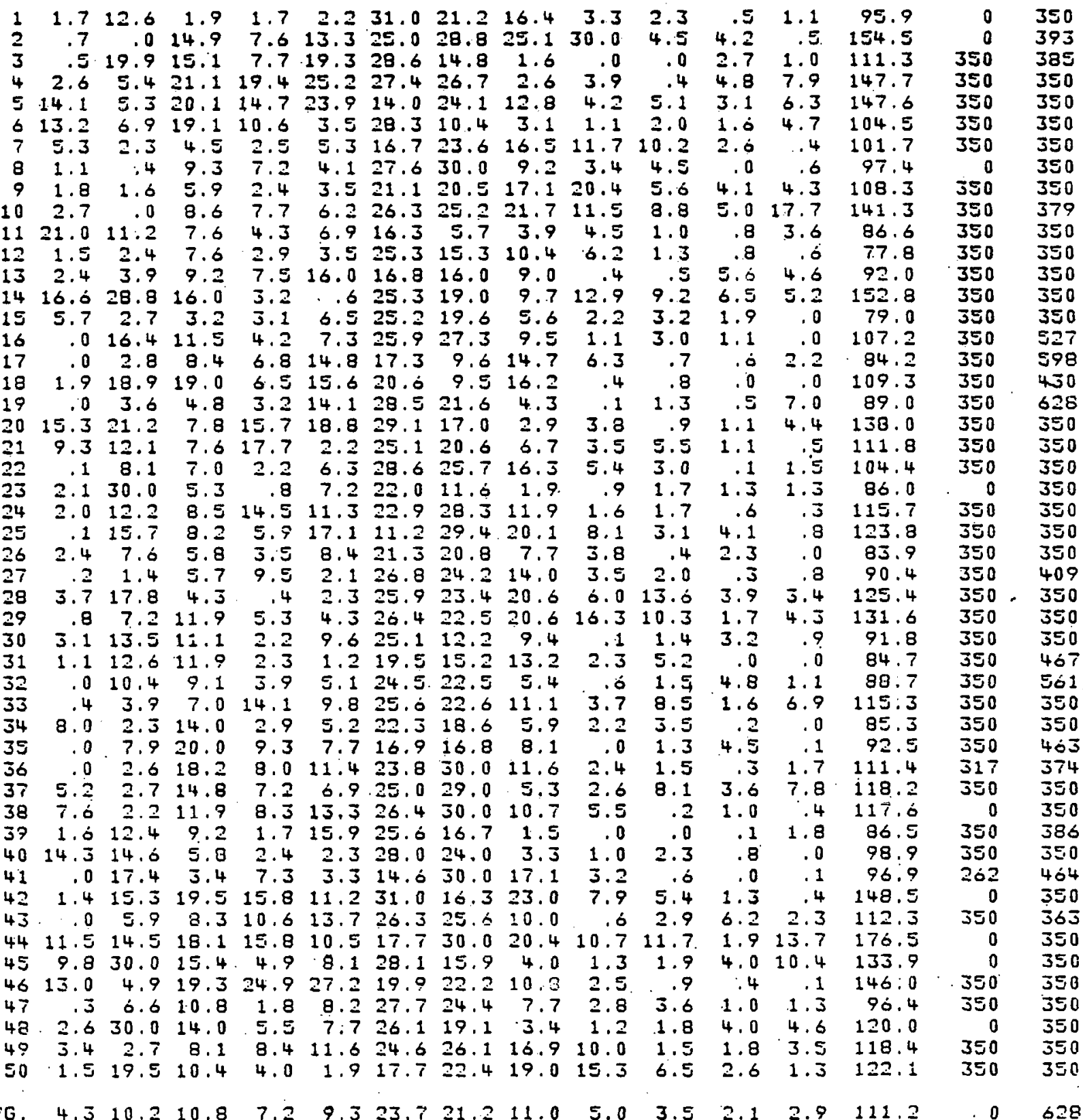


JATERVLLET RESERVOIR HYGRO-ELECTRIC STUUY EASEI ON SIREGHFLOW SYNTHESIIE

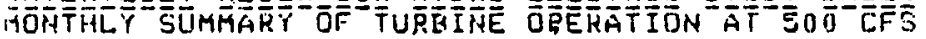

ASSUMING : OPERATION CEASES AT 3 FEET BELOW FLASHEOARIIS

UOMESTIC WATER. SUPPLY MAINTAINEII AT . MGI

DEFICIT $C M G$

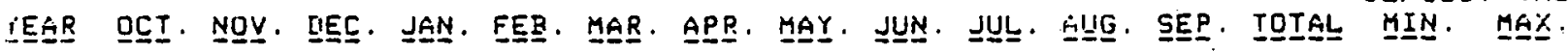

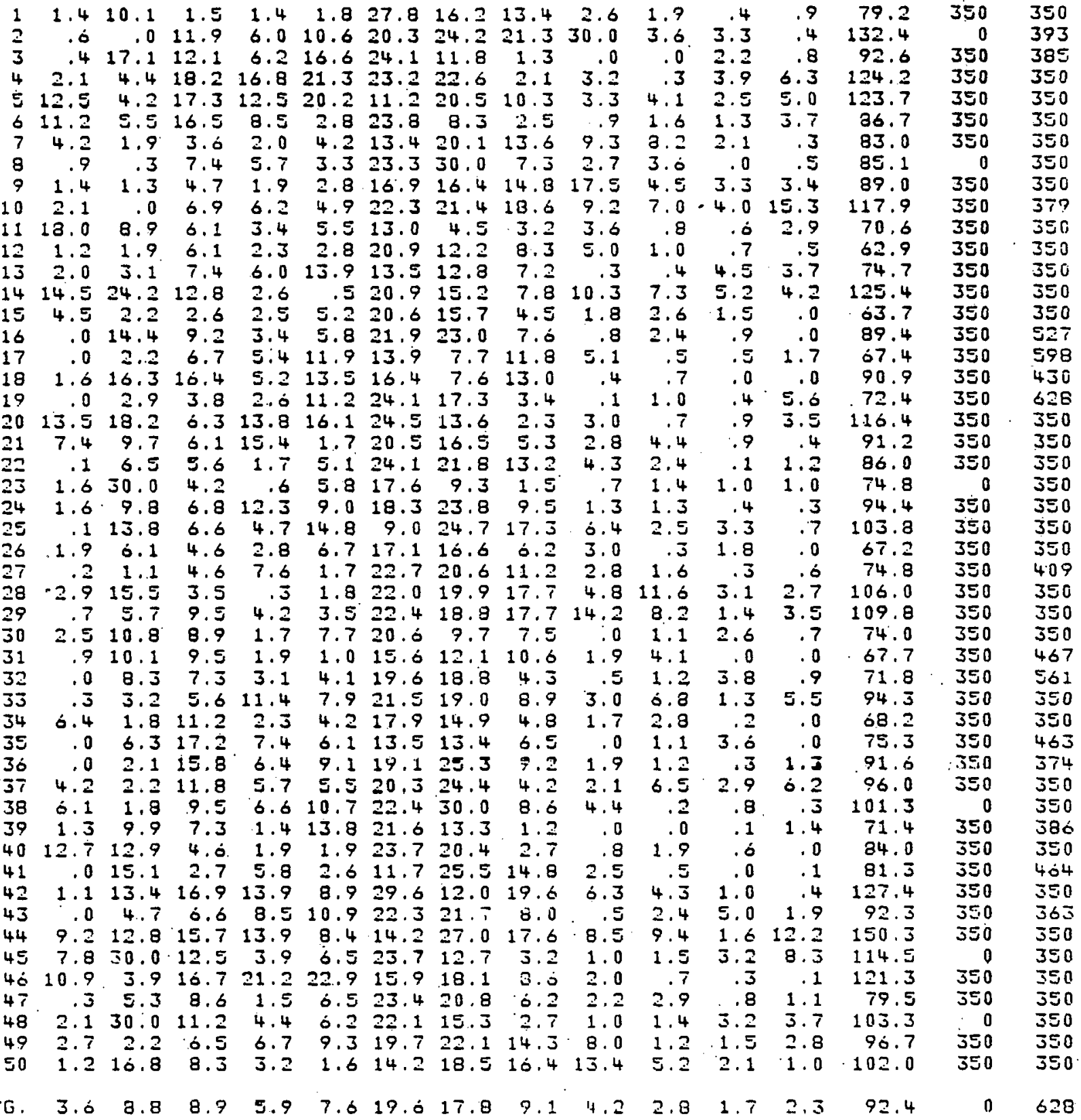


JGTERVLIET RESERVDIP HYURO ELECTRIC STUEY BASED OH SIPEAMFLOW SYNTHESIS

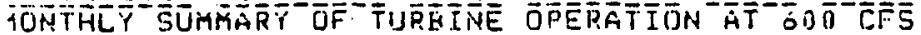

ASSUMING: DPERATION CEASES AT 3 FEET BELOW FLASHEOARIIS

DOMESTIC WATER SUPPLY MAINTAINED AT 6 MGI

DEFICIT (MG

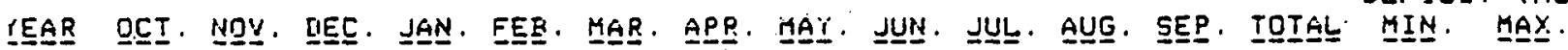

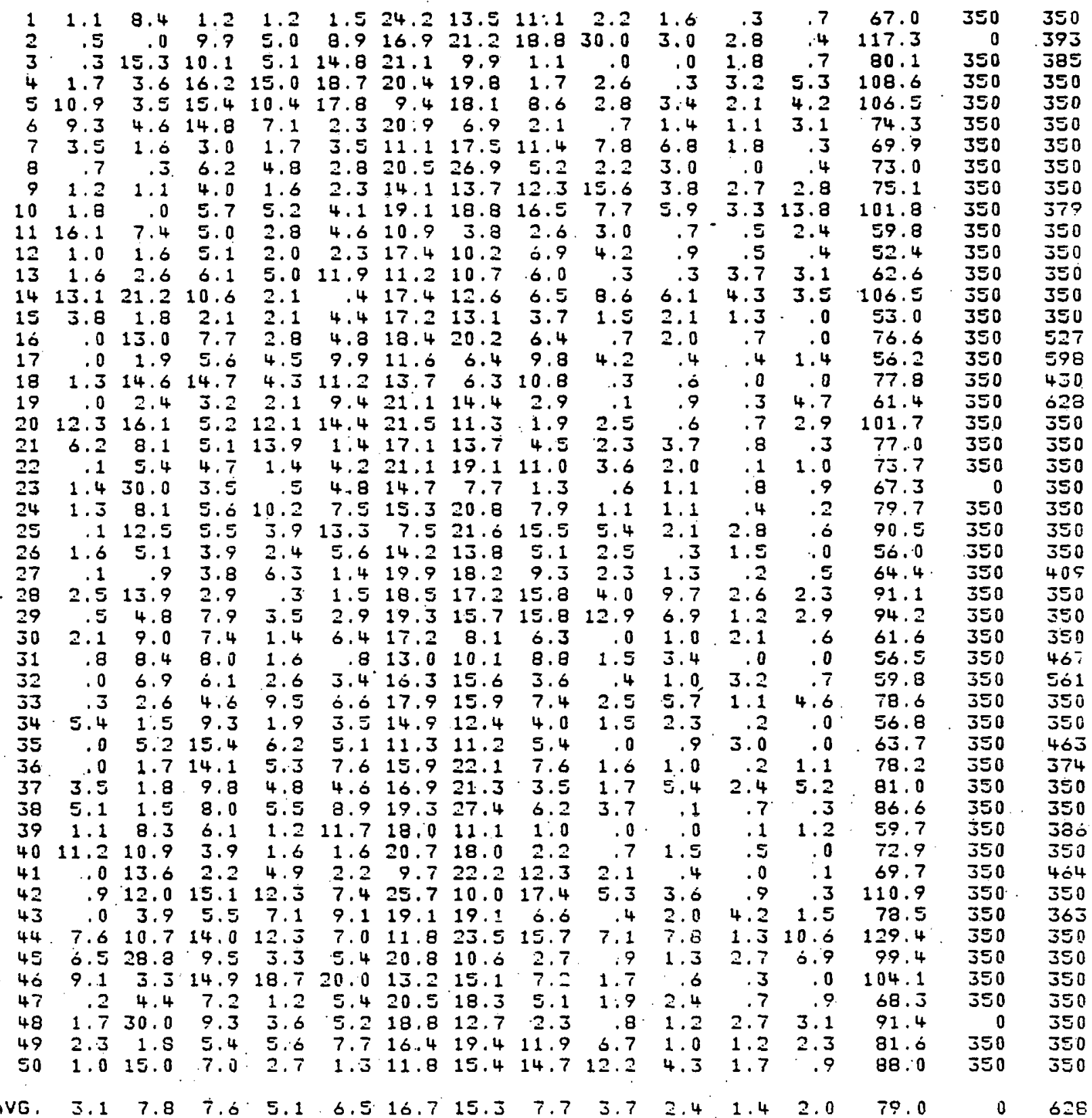




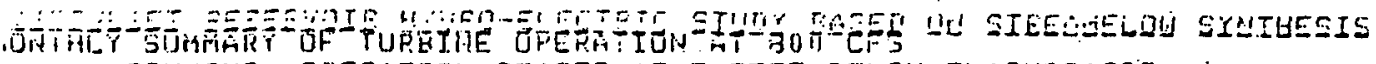
GSSUMING : OPERATIOH CEASES AT 3 FEET BELOW FLASHGDARDS

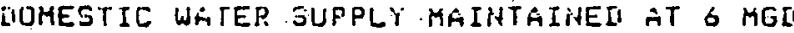

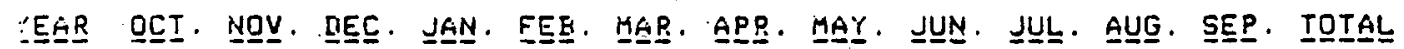

DEFICIT \&MG

\begin{tabular}{|c|c|c|c|c|c|c|c|c|c|c|c|c|c|c|c|}
\hline 1 & .3 & 6.3 & .9 & .9 & 1.1 & 19.7 & 10.1 & 9.4. & 1.0 & 1.2 & .3 & 0 & $51: 8$ & 350 & 35 \\
\hline & .4 & & 7.4 & 3.8 & 6.6 & 12.7 & 17.4 & 15.6 & 26.4 & 1.6 & 2.1 & 7 & & 350 & \\
\hline & .2 & 13.0 & 7.5 & 3.9 & 12.5 & 17.2 & 7.4 & .8 & .0 & .0 & 1.4 & .5 & 64. & & \\
\hline 4 & 1.3 & .2 .7 & 13.7 & 12.8 & 15.4 & 15.7 & 16.4 & 1.3 & $2 \cdot 0$ & .2 & 2.4 & 4.0 & 87 & & \\
\hline 5 & 8.2 & $\begin{array}{l}2.6 \\
3.5\end{array}$ & $\begin{array}{l}12.9 \\
11.8\end{array}$ & $\begin{array}{l}7.3 \\
5.3\end{array}$ & $\begin{array}{r}14.8 \\
1.7\end{array}$ & $\begin{array}{r}7.0 \\
16.8\end{array}$ & $\begin{array}{r}13.8 \\
5.2\end{array}$ & $\begin{array}{l}6.4 \\
1.5\end{array}$ & $\begin{array}{r}2.1 \\
.6\end{array}$ & $\begin{array}{l}2.6 \\
1.0\end{array}$ & $\begin{array}{r}1.6 \\
.8\end{array}$ & $\begin{array}{l}3.1 \\
2 . \frac{1}{3}\end{array}$ & & & \\
\hline 7 & 2.7 & 1.2 & 2.3 & 1.2 & 2.6 & 8.4 & 13.1 & 8. & 5.8 & 5.1 & 1.3 & .2 & & & \\
\hline & .5 & .2 & 4.6 & 3.6 & 2.1 & 10.0 & 21.7 & 3.9 & 1.7 & 2.2 & .0 & 3 & & & \\
\hline & .9 & .8 & 3.0 & 1.2 & 1.7 & 10.6 & 10.3 & 9.2 & 13.2 & 2.8 & 2.0 & 2.1 & & & \\
\hline & 1.3 & .0 & 4.3 & 3.9 & 3.1 & 14.3 & 15.2 & 13.9 & 5.8 & 4.4 & 2.5 & 11.8 & & & \\
\hline & 13.6 & 5.6 & 3.8 & 2.1 & 3.5 & 3.2 & 2.8 & 2.0 & 2.2 & .5 & .4 & 1.8 & & & \\
\hline &. a & 1.2 & 3.8 & 1.5 & 1.7 & 13.0 & 7.0 & 5.2 & 3.1 & .6 & .4 & .3 & & & \\
\hline & 1.2 & 2.0 & 4.6 & 3.8 & 8.9 & 3.4 & 8.0 & 4.5 & .2 & .3 & 2.8 & 2.3 & & & \\
\hline & 11.2 & 17.4 & 8.0 & 1.0 & .3 & 13.1 & 9.5 & 4.9 & 0.4 & 4.6 & 3.2 & 2.6 & & & \\
\hline & 2.8 & 1.4 & 1.6 & 1.6 & 3.3 & 12.9 & 9.8 & 2.8 & 1.1 & 1.6 & 1.0 & .0 & & & \\
\hline & .0 & 10.1 & 5.8 & 2.1 & 3.6 & 13.8 & 16.0 & 4.8 & .5 & 1.5 & .5 & .0 & & & \\
\hline & .0 & 1.4 & 4.2 & 3.4 & 7.5 & 8.7 & 4.8 & 7.3 & 3.2 & .3 & .3 & 1.1 & & & \\
\hline & $1: 0$ & 12.4 & 11.0 & 3.2 & 8.4 & 10.3 & 4.8 & 8.1 & .2 & .4 & .0 & .0 & & & \\
\hline & .0 & 1.8 & 2.4 & 1.6 & 7.0 & 17.1 & 10.8 & 2.1 & 1 & & & 3.5 & & & \\
\hline & 9.0 & $1 \overline{5} .6$ & 3.9 & 9.1 & 12.2 & 17.7 & 8.5 & 1.4 & 1.9 & .4 & .5 & 2.2 & & & \\
\hline & 4.6 & 6.0 & 3.8 & 11.7 & 1.1 & 12.8 & 10.3 & 3.3 & 1.7 & 2.8 & .0 & .2 & & & \\
\hline & .1 & 4.1 & 3.5 & 1.1 & 3.2 & 17.2 & 15.8 & 3.2 & 2.7 & 1.5 & .1 &. & & & \\
\hline & 1.0 & 26,7 & 2.0 & .4 & 3.0 & 11,0 & 5.8 & .9 & .5 & $=$ & $\therefore$ & .6 & & & \\
\hline & 1.0 & 0.1 . & 4.2 & 7.7 & 5.0 & 11.5 & 17.1 & 5.9 & .8 & .8 & & & & & \\
\hline & .0 & 9.6 & 4.1 & 3.0 & 10.3 & 5.6 & 17.7 & 12.9 & 4.0 & 1.6 & 2.1 & 4 & & & \\
\hline & 1.2 & 3.8 & 2.9 & 1.8 & 4.2 & 10.7 & 10.4 & 3.7 & 1.9 & .2 & 1.1 & c & & & \\
\hline & .1 & .7 & 2.9 & 4.7 & 1.0 & 15.0 & & 7.0 & 1.7 & 1.0 & .2 & & & & \\
\hline & 1.8 & 11.7 & 2.2 & .2 & & & & 13.4 & 3.0 & 7.3 & 1.9 & 1. & & & \\
\hline & .4 & 3.6 & 5.9 & 2.6 & 2.2 & 14.5 & 11.8 & $13.4^{\circ}$ & 10.4 & 5.1 & .9 & 2. & & & \\
\hline & 1.5 & 6.8 & 5.6 & 1.1 & 4.8 & 12.9 & o. 1 & 4.7 & .0 & .7 & 1.0 & .5 & & & \\
\hline & .6 & 6.3 & 6.0 & 1.2 & .6 & 9.7 & 7.6 & 0.0 & 1.2 & 2.6 & 0 & & & & \\
\hline & .0 & 5.2 & & 2.0 & 2. & 12.2 & 11.7 & $2: 7$ & 3 & & 2.4 & & & & \\
\hline & .2 & 2.0 & 3.5 & 7.1 & 4.9. & 13.4 & $11.0^{\circ}$ & 5.0 & 1.8 & 4.3 & .8 & 3.4 & & & \\
\hline & 4.0 & 1.1 & 7.0 & 1.5 & 2.0 & 11.2 & 9.3 & 3.0 & 1.1 & 1.8 & .1 &. & & & \\
\hline & .0 & 3.9 & 12.8 & 4.6 & 3. & 8.5 & 8. & 4.1 & .0 & $\cdot$ & 2.3 & 1 & & & \\
\hline & .0 & 1.3 & 10.6 & 4.0 & 5. & 11.9 & 18. & 5.7 & 1.2 & .8 & .3 & & & & \\
\hline & 2.6 & 1.4 & 7.4 & 3.6 & 3.4 & 12.7 & 17.5 & 2.6 & 1.3 & 4.1 & 1.8 & 3.3 & & & \\
\hline & 3.8 & 1.1 & 0.0 & 4.1 & 6.7 & 14.5 & 22.1 & $4 . \overrightarrow{3}$ & $2 . \theta$ & $\cdot 1$ &. .5 & .3 & & & \\
\hline & .8 & 6.2 & 4.6 & .9 & 8.8 & 13 & 8.3 & .8 & .0 & .8 & .1 & . & & & \\
\hline & $8: 4$ & $8: 2$ & 2.9 & 1.2 & 1. & 10.5 & 13. & 1.7 & .5 & 1. & .4 & 8 & & & \\
\hline & .0 & 11.5 & 1.7 & 3.0 & 1.0 & 7.3 & 18.2 & 9.2 & $1: 0$ & . & .0 & 1 & & & \\
\hline & .7 & 9.0 & 12.3 & 5.2 & 5.6 & 20.8 & 7.5 & 14.6 & $3 . ?$ & 2. & -.0 & .3 & & & \\
\hline & .0 & 2.9 & & 5.3 & & 14 & 15.6 & & .3 & 1. & 3.1 & 1. & & & \\
\hline & 5.7 & 8.0 & 10.5 & 9.3 & 5.3 & 8: & 19.1 & 13.3 & 5.3 & 5.8 & 1.0 & 7. & & & \\
\hline & 4.9 & 23.1 & 7.1 & 2.5 & 4.1 & 10.0 & 7.9 & 2.0 & .ó & 1.0 & 2. & 5. & & & \\
\hline & 0.8 & 2.5 & 12.0 & 15.6 & 16.4 & 9.9 & 11. & 5.4 & 1.3 & . & . & • & & & \\
\hline & .2 & 3.3 & 5.4 & & 4. & 10.1 & 14.2 & 3.8 & 1.4 & $1: \varepsilon$ & .5 & . & & & \\
\hline & $1 . \overline{3}$ & 30.0 & 7.0 & 2.7 & 3.9 & 14.1 & 9.5 & 1.7 & & .8 & 2.0 & 2.9 & & & \\
\hline & 1.7 & 1.3 & 4.0 & 4.2 & 5.8 & 12.3 & 16.1 & 8.9 & 5.0 & & .9 & $i$. & & & \\
\hline & .7 & 12.6 & 5.2 & 2.0 & 1.0 & 8.9 & 116 & 11.0 & 9.1 & 3.3 & 1.3 & .7 & 68.1 & 350 & \\
\hline & .4 & & . & 3.9 & 5.0 & 12.8 & 1.9 & 6.0 & 2.9 & 1,8 & $1: 1$ & 1.5 & 51,3 & & \\
\hline
\end{tabular}


AVERAGE ANNUAL POTENTIAL POWER PRODUCTION

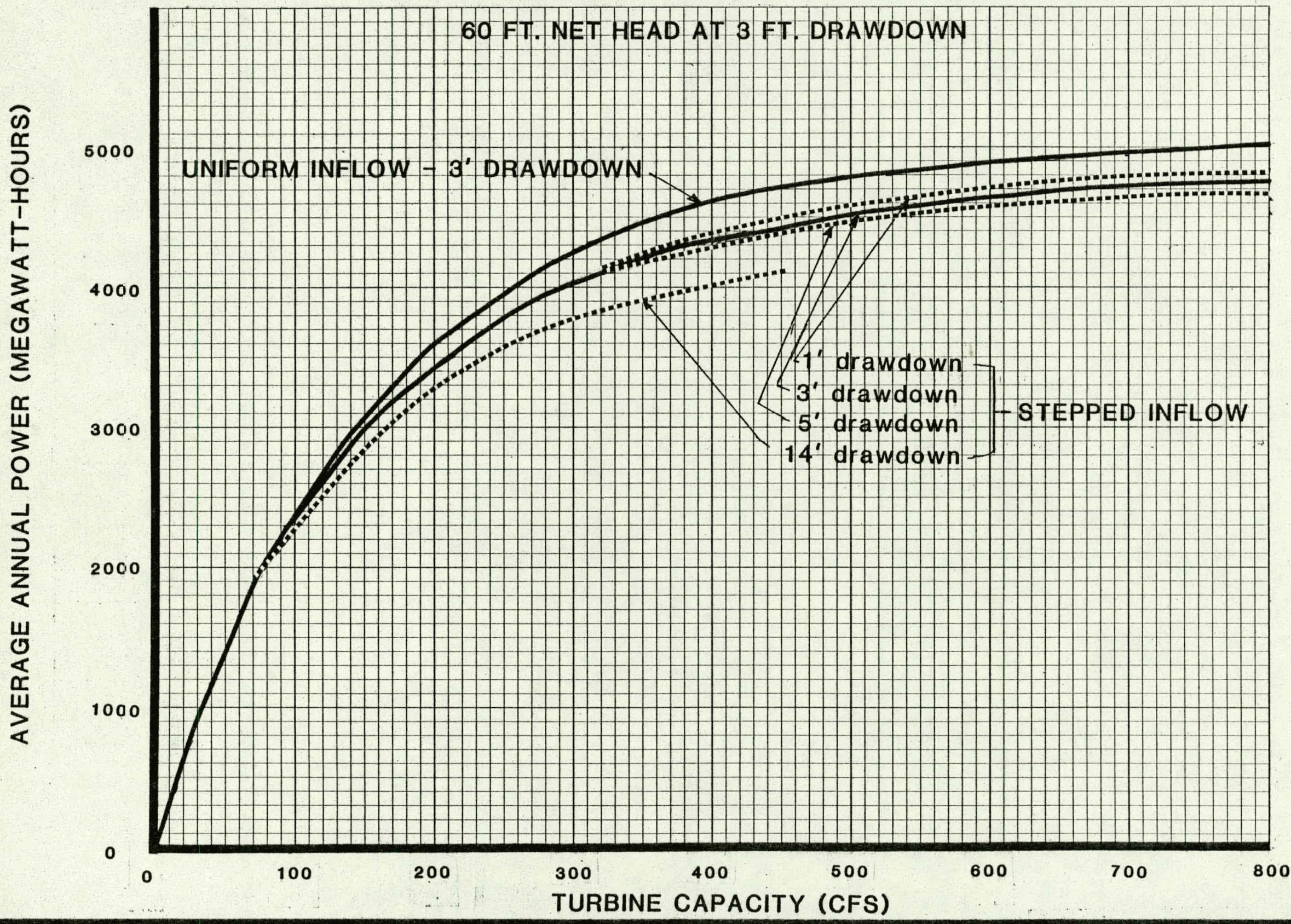




\section{8}

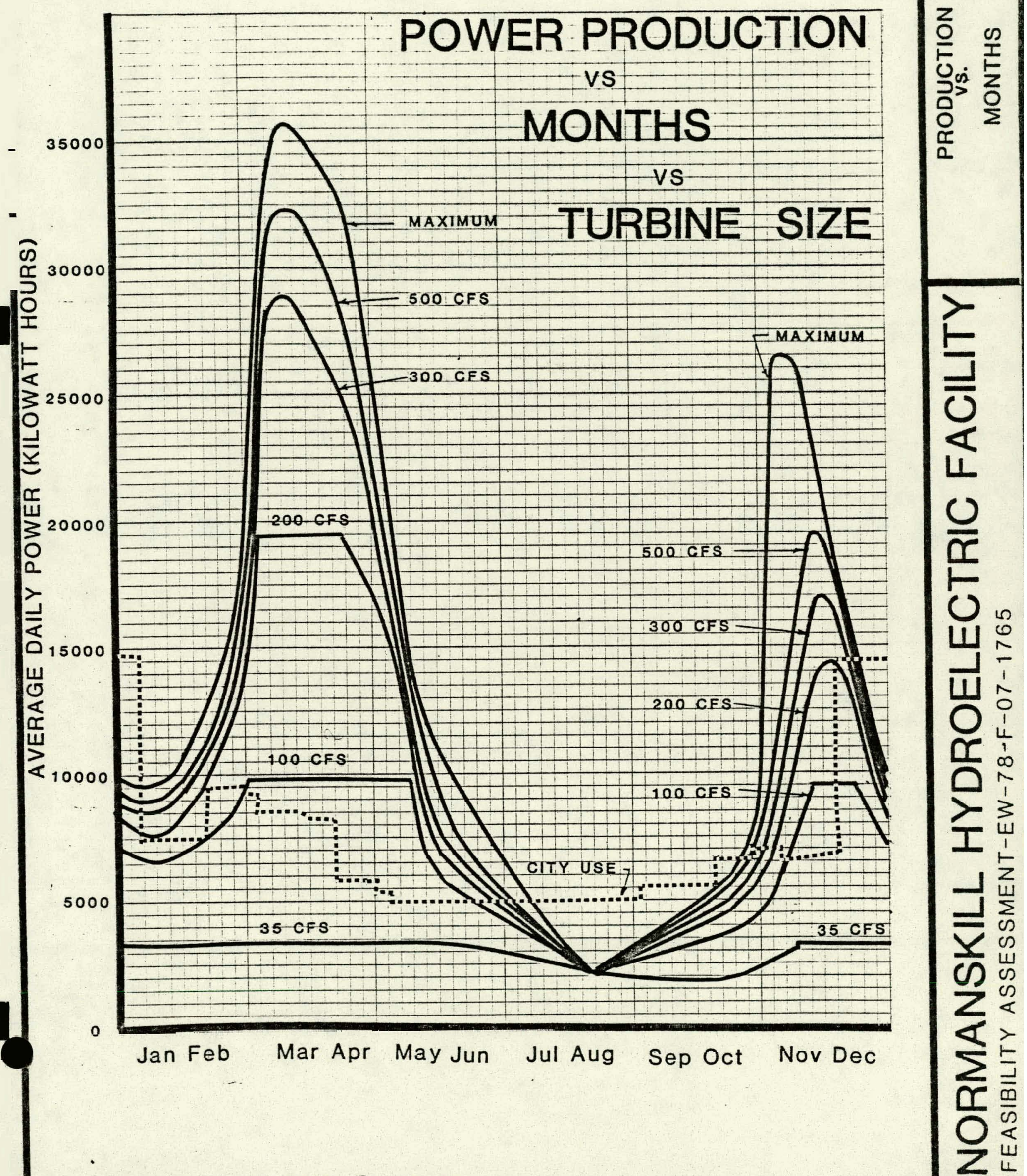

GIVEN: 60 FT. NET HEAD @ .80 eff. 


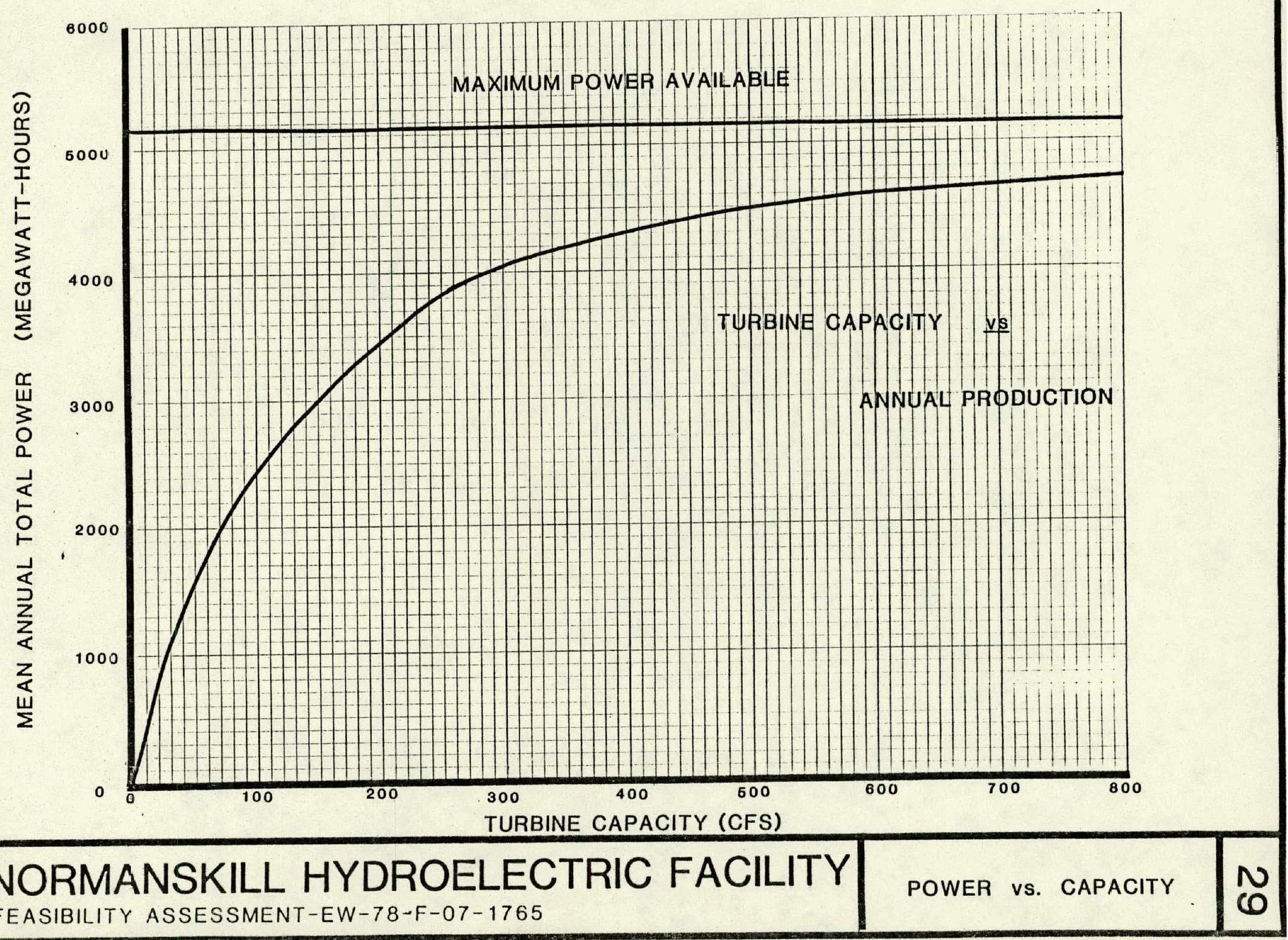


MARKETING

Electric Power Market

The primary consideration of the marketing analysis has been the determination of suitable placement of any surplus power generated at any proposed hydroelectric facility at French's Mills. It was assumed at the outset that the highest benefit for the first incremental power generated would be for municipal use at the site to replace the mechanical hydropower presently utilized for water supply pumping with electrical power. Placement of the power generated in excess of this requirement was considerably more involved.

It was first necessary to determine what legal restrictions would be placed upon disposal of the surplus power. Discussions were held with the New York state Public Service Commission. It was determined that under terms of current PSC tariffs and regulations, sale of surplus power by the City of Watervliet to any existing consumer within the Niagara Mohawk Power Corporation franchised area would be considered "pirating" and would be illegal, unless the City, as producer, could prove this would be in the public interest. New consumers are excepted from this rule and a consumer, requesting a new service, may possibly be served by the developer of the Normanskill Hydroelectric facility. Since firm power generated at the site would be minimal, and it would serve at best only to supply the City's municipal pumping station, it was obvious that sale of power to new consumers as their sole source of power would be unacceptable. For this reason, discussions with existing or potential new retail consumers such as clients of the Guilderland Industrial Development Agency, were not pursued.

It was determined, that the PSC pirating regulations did not apply to the possible sale of power either to the Power Authority of the state of New York (PASNY) or to Federal facilities such as the U.S. Government Arsenal at Watervliet. In this light, discussions were held with the Watervliet Arsenal to determine the interest on their part to using municipally generated power. Due to the large 
amount of power consumed by the Arsenal in their manufacturing facilities in relation to what the City could supply, this alternative was judged to be unfeasible.

The Power Authority of the state of New York (PASNY) is a state-chartered authority whose purpose is to develop electrical generation and transmission facilities within the state. A request has been made of PASNY to ascertain their interests in purchasing the surplus dump power available from the Normanskill Hydroelectric facility. As of this date, a formal reply from this organization has not been received.

Discussions were held with the Niagara. Mohawk Power. Corporation (N.M.P.C.) to explore several areas of interest. The first area explored with the utility was the possibility that N.M.P.C. purchase all surplus power from this facility. Discussions with the local N.M.P.C. management yielded an offer from Niagara Mohawk Power Corporation of 3 to 5 mils per kilowatt hour for this surplus power.

Pursuing the determination of suitable purchase price, an examination was made of contracts on file with the New York State Public Service Commission between the Niagara Mohawk Power Corporation and various municipal and private producers of electrical energy. Detailed examination was made of these contracts; both for prevailing rate and also of their interface requirements. Two of the five existing contracts most comparable to our case, established the value of power at $4.5 \mathrm{mils}$ per kilowatt hour, the other three at $9 \mathrm{mils}$ per kilowatt hour. Terms in three of the contracts were left indefinite and the remaining two expire in 1983. All the most current contracts executed between February and June of 1978 set a price for this power at 9 mils per kilowatt hour. In certain cases, these contracts provide for reciprocity on the part of Niagara Mohawk for supply of power to the municipality in the event that their generation facilities were off-line at the rate of 1.2 cents per kilowatt hour. A final determination was made based upon our discussions and examinations of current contracts and on evaluation of current energy prices on national and state level 
that the $9 \mathrm{mil}$ per kilowatt hour rate would be used for calculating benefits accruable to the city for sale of surplus power to Niagara Mohawk.

The second area of discussion with the utility (N.M.P.C.) involved determination of charges imposed by the utility for "wheeling" power. "Wheeling" involves transmission of power generated at the Normanskill Hydroelectric site over existing Niagara Mohawk Power Corporation facilities. Power would then be extracted within the City of Watervliet at municipal facilities. The charge for "transmission" of municipally generated power would take into account transmission losses and expenses the utility would incur in operating and maintaining that portion of their transmission system allocable to this use. It should be noted here that transmission system is used in a generic sense since transmission of the quantity of power expected to be developed with the Normans Kill site could be easily handled by the utility's distribution system. The utility, through its local management, showed a very cool initial reception to even discussing wheeling. Subsequent discussions with the syracuse office yielded a tentative offer of 2 to $4 \mathrm{mils}$ per kilowatt hour for wheeling. Presently, the Power Authority of the State of New York (PASNY) is charged 2 to 2-1/2 mils per kilowatt hour for wheeling across N.M.P.C. Iines on an aggregate state-wide basis. It is expected that this rate will be increased shortly at the insistence of the Public service Commission. It has also been determined that the Federal Energy Regulatory. Commission could be addressed to compel $\mathrm{Niagara}$ Mohawk to wheel power to the City if an agreement on wheeling could not be reached with Niagara Mohawk Power Corporation. A similar situation arose in the Town of Massena, New York.

Final discussions were held with the Niagara Mohawk Power Corporation on the possibility of purchase or lease of the site by Niagara Mohawk for construction of generation facilities by the utility. Niagara Mohawk had initially expressed some interest in possible lease of the site but subsequently has stated informally that they might rather, purchase the site outright. No further discussions have been held with Niagara Mohawk regarding this matter. 
Having determined the external costs and benefits to the city for generation and sale of power from the proposed site, determination of the intrinsic value of power remained.

\section{Municipal Power Consumption}

The detailed account of power purchased by municipal

facilities was undertaken to determine what possible benefits would accrue to the city for use of surplus power available at the site using the assumption that the power would be wheeled across Niagara Mohawk Power Corporation facilities. An audit was performed to determine the amount of energy consumed, it's total cost and periods of consumption. All city accounts with the Niagara Mohawk Power Corporation were compiled for a three year span (1976-1978) for 13 municipal facilities listed as follows:.

1. Municipal Swimming Pool

2. Water Filtration Plant

3. City Hall

4. Water Distribution Pumping Station

5. City Garage

6. Municipal Ice Skating Facility

7. Raw Water Pumping Station

8. Senior Citizen Center

9. Fire Station

10. Street Light Service

11 Traffic Light Service

12. Civic Center

13. Hudson Shores Park

Data was compiled on the electrical consumption of these facilities by taking monthly or bimonthly utility bills and disaggregating the total consumption over the billing period. In certain cases, as in the case of the municipal ice skating facility, adjustments were made for known peak operating loads from facility operating records. In the case of the Senior Citizen Center, now under construction, engineer's estimates of power requirements at the facility were utilized in determining total power consumption for a typical year. 
A significant factor in the City of Watervliet's annual cost for power is the operation of the City's street lighting system. The street lighting system is comprised of approximately 598 luminaires both separately mounted and mounted on utility company poles. Under the present system, the City pays an annual charge for each fixture as defined by the New York state Public Service Commission Tariff Classifications. (P.S.C. No. 213)

The total annual charges for the street lighting facilities is then divided by the calculated load based upon manufacturer's catalogue power requirements for the individual lamps. A factor of 4,170 hours per year is used for the anticipated on-time of luminaires activated by photocells. The monthly charge billed, therefore, is approximately $1 / 12$ th of the annual charge, but varies according to the time of the year representing the normal variations of the nocturnal cycles. On a straight calculated kilowatt-per-year basis, the cost for this service is approximately $7-1 / 2$ cents per kilowatt hour. It is understood, however, that this cost represents not only cost of power to the equipment, but also operating maintenance costs and an amount sufficient to amortize the facilities over their expected life. Under terms of the present contract between the City and Niagara Mohawk Power Corporation, under PSC Tariff No. 213, the city may at any time elect to terminate service by paying the depreciated book value of the facilities less the salvage value. With this in mind, an attempt was made to determine this residual value as a means of calculating the true benefit for either power consumption alone or full operation of the facilities by the city. Repeated requests to Niagara Mohawk Power Corporation for this information have been futile. The only figure offered by N.M.P.C. verbally was an. approximate replacement value of "about $\$ 100,000$." It was also inferred that this replacement value included only materials. Upon further investigation, several apparent discrepancies have been found in the current contract between the City and Niagara Mohawk Power Corporation. Prior to July 1977, the majority of lighting fixtures listed on N.M.P.C contract schedule SL, as installed within 
the City of Watervliet, were of conventional incandescent type. Billing costs, prior to this date, were calculated on the filed tariff charges for this type of luminaire. However, the new Schedule SL furnished the City by N.M.P.C. after July 1977 indicates that all of the approximately 525 luminaires were either mercury vapor or high pressure sodium type. Subsequent billing for street lighting facilities has been on this basis. Inquiries to the Niagara Mohawk Power Corporation as to when and if the entire city street lighting system was converted to the newer type of luminaire have been to no avail. A cursory examination of several street lighting fixtures indicates that they are in fact the old style incandescent luminaire. Having been impeded in our attempts to obtain sufficient information from the Niagara Mohawk Power Corporation to determine the benefits and costs for city ownership and operation of the street lighting facilities, the costs and benefits were developed independently. As a basis of capital cost for take-over of the facilities, the $\$ 100,000$ quoted by Niagara Mohawk (thought to be excessively high) was used. An estimate from a local reputable contractor with considerable experience in installation, operation and maintenance of municipal street lighting facilities was obtained for the installation portion of this capital expense. In addition, an estimate for contract operation and maintenance (relamping, etc.) was also obtained. Using these figures and amortizing the capital portion of the outlay over 20 years at the current city bonding rate, it was determined that the annual charges including operation and maintenance for the existing street lighting facilities was approximately $\$ 25,000$ per annum. The difference then between this figure and the $\$ 50,000$ per year current charges to the city from Niagara Mohawk Power Corporation was used therefore as representing the electrical power consumption cost of the street lighting facilities.

A summary of electrical power consumption in the facilities investigated is shown on Figure 30 . 


\section{Benefit Value Program}

Each of the various alternative hydroelectric generating configurations which were considered would produce electric power as its primary benefit. The value of the power which it is capable of producing depends not only on the total amount of power, but also on whether the power that is being produced will be simultaneously consumed by the water pumping station at the site, whether the remaining excess, if any, will simultaneously be used remotely by municipally owned facilities in the City of Watervliet, or whether the remaining excess, if any, will be "dumped" into the Niagara Mohawk system.

A model was developed which compared the time and rate distribution of the power which could be produced against the power consumption requirements of the owner both at the site and within the City. The electrical energy consumption pattern for a recent year was input to the model. It summarized the annual use by representing each month by two periods, each with relatively uniform use. For each of those 24 usage periods, the power capable of being produced by each alternative configuration for the 50 corresponding months from the reservoir streamflow simulation was determined and the average amount of power falling into each of the three benefit categories was compiled. The simulation thus progressed through the 24 calendar periods and was summarized as annual totals to which the appropriate benefit rates are applied in order to derive the total annual value of the power generated for each alternative and in each benefit category.

When the generating equipment may be idle due to lack of available water, the generating equipment could have a fourth potential marketable benefit to the Niagara Mohawk Power Corporation if operated in a mode by which it could provide power factor correction in the local Niagara Mohawk service area. The value of such potential benefit would be proportional to the peak generating capacity of the machine and might accrue whenever it is not generating electricity. A nominally low benefit rate was chosen to reflect the remoteness of this aspect as a factor for selection. 
The credit summary was further refined to reflect the fact that any of the Type 3 turbines (Kaplan, see Figure 44) would be capable of operating at load factors or flow rates reduced to approximately $10 \%$ of the peak capacity. A correction was further applied to the benefits determined for these turbines to reflect the advantageous fact that they could operate a substantially greater percentage of time compared to that calculated under the assumption that the turbine could run only at full flow capacity. Total power output for the generating equipment would not be increased, but a greater relative amount of the power which it could produce would be capable of being used simultaneously by the pumping station at the site accruing power credit at a higher rate and avoiding the necessity to put this power into the Niagara Mohawk Power Corp. system incurring a transportation or "wheeling" charge for the energy. The correction for increased operating time is based upon the relationship shown in Figure 25. Credits for power factor correction were correspondingly reduced for all of the Type 3 turbines in proportion to decreased idle time.

Figure 30A displays an annual summary for each alternative showing the total city power consumption as well as the cost at the assumed rate per kilowatt hour and the corresponding average of credits over the 50-year simulation period for power produced in each of the benefit categories and the resulting net cost of power after applying those credits.

The total credits thus derived are the basis for several later computations pertaining to machine selection and overall economic feasibility.

\section{Utility Interface}

Certain technical requirements for interfacing with Niagara Mohawk Power system were determined throụgh discussions with Niagara Mohawk facility engineers. The transmission voltage for input into the Niagara Mohawk system at the French's Mills location would be 13,200 volts. It was assumed that although present Niagara Mohawk facilities at the site are of inadequate construction for 
transmission of this power, current planning requirements already include extension of 13,200 volt facilities to the site in the near future.

Requirements for protection and relaying used are those stated in IEEE-357-1973 "IEEE Guide for Protective Relaying of Utility Consumer Interconnections". Niagara Mohawk Power Corporation has also been approached to determine the feasibility of using power factor correction during off-line generator periods. Since there is as a conservative estimate, over 600 horsepower of connected inductive load within a one mile radius of the proposed generation facilities, the benefits of power factor correction would seem substantial. While mild interest has been expressed by Niagara Mohawk concerning this power factor correction, no value for this benefit has been determined. For this reason, a nominal token value has been shown in the calculations purely to represent the possibility of this end use.

A final interface requirement discussed has been that of concurrency of power generation and consumption. The accounting methods which would be used to calculate the payment amounts accruable either to Niagara Mohawk or to the City for power either generated, sold, purchased or wheeled will involve metering at some 14 locations. Since the proposed facility most probably will be a run-of-river facility with normal fluctuations in generating pattern, some conditions must be established between the utility and the municipality as to the concurrency of generating and consumption periods. While the most obvious accounting methods would be the one presently in use, that is, simple monthly readings of pertinent meters with the subsequent charges or credits for power either sold, used or wheeled, this method may not be entirely fair to the utility. Should the hydroelectric facility for instance, generate at a maximum rate for the first week of the month and fall to a low or zero rate for the remaining period in the month, the city could conceivably have credit for the power generated averaged over the month. Preliminary discussions with the utility have indicated that they would be amenable to any equitable accounting stance which 
would take these concurrency problems into consideration. Consideration of this concurrency problem has lead to consideration of a fairly sophisticated operating system for a facility of this size in order to provide the optimum operating mode within the accounting periods to maximize City benefits.

Incurred Municipal Benefits and Costs

Additional economic factors relating to city development of the project were determined under this chapter. Factors which must be considered are changes in property tax assessments, insurance requirements, labor requirements and municipal bonding rates if municipality is to assume financial responsibility for the facility. Currently, the city pays approximately $\$ 31,000$ in school, county, town, highway, and fire district taxes to the Town of Guilderland and the appropriate school and fire districts for property owned at the Town of Guilderland site. The present equalization rate for construction of the type considered by this project is $10 \%$. Based upon a current tax rate of $\$ 255$ per $\$ 1,000$ of assessed valuation, $\$ 1,000,000$ of construction would increase the city's present tax burden by approximately $\$ 26,000$ per year. A figure somewhat less than this was used in the Economic Analysis of the project with the assumption that assessment would not be at full value of construction since substantial portions of the construction involve modification or rehabilitation of existing facilities.

Presently, the municipal pumping station located at the proposed site is manned 24 hours a day, 7 days per week. It is assumed that generation of hydroelectric power at the site would additionally entail modification of the present pumping station to serve as the control facility. This facility would, for safe and adequate control, be highly automated. A reduction in workforce to only one or two individuals from the present staff of six would be possible. These individuals would be responsible for monitoring the operation and maintaining the combined hydroelectric/pumping facility. At current levels, the cost to the city is approximately 
$\$ 19,000$ per year per man in wages and fringe benefits. Substantial savings would accrue to the city with the reduction in the labor force if the proposed hydroelectric facility is pursued. Presently, the city carries sufficient Workmen's Compensation, Property and Public Liability insurance at the site to meet Municipal Law requirements. It has been assumed that any additional insurance requirement due to the construction of a hydroelectric facility at the site would be self-insured. Further discussion of the amount assumed for payment into a sinking fund for this use is discussed in the Economic Analysis section.

Since at least a portion of the proposed facility would be financed through municipal borrowing, a determination of expected bonding rates was made. The actual rate for municipal bonding by the City of Watervliet, based upon geometric mean of the past five years' issuance of bonds, is $6.31 \%$. Further discussion of the ramifications of municipal bonding will be given in the Implementation section. 


\section{MUNICIPAL ELECTRIC CONSUMPTION}

MUNICIPAL ELECTRICAL CONSUMPTION

FOR TYPICAL CALENDER YEAR BY FACILITY

EXPRESSED AS KWH PER DAY

ANNUAL SUMMARY SHOWN

FOLLOWING DECEMBER

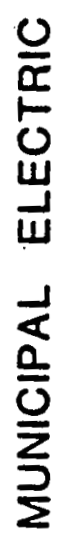

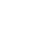


CITY OF WATERVLIET

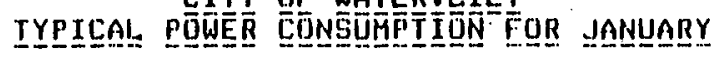

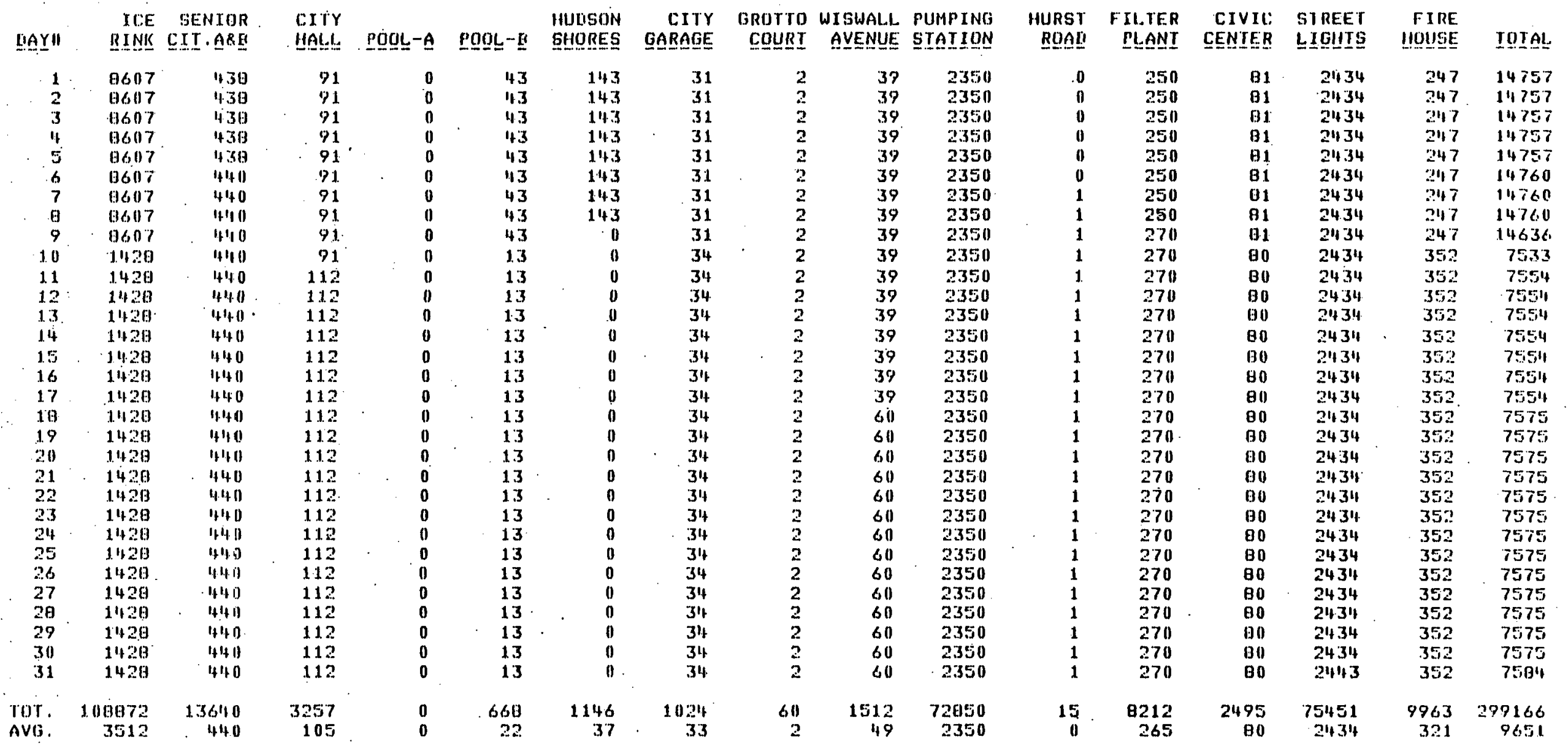


CITY OF WATERVLIEI

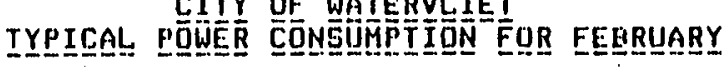

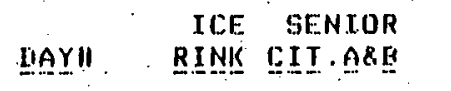

CITY

HALL. POOL-A POOL-B.

HULISON
SITY GROTTO WISWALL PUMPING
GHORES $\begin{array}{lll}1428 & 440 & 112 \\ 1428 & 440 & 112 \\ 1428 & 4+40 & 112 \\ 1428 & 440 & 112 \\ 1428 & 4140 & 112 \\ 1429 & 440 & 112 \\ 1428 & 440 & 112\end{array}$

$\begin{array}{lll}1428 & 4110 & 112 \\ 3516 & 4110 & 112\end{array}$

$3516 \quad 1440=99$

$3516 \quad 410 \quad 90$

3516 - 1110 99

$3516 \cdot 4+40$

$3516, \quad 4110$

$3516 \quad 1,40$

$3516 \quad 4+16$

$3516: 4140$

$3516 \quad 1140$

3516 ind

$3516 . \quad 4+11$

$3516 \quad 4140$

$3516 \quad 1140$

35its $4+40$

$3.160 \quad 11 \%$

$3516 \quad 1140$

$3 . .16$

3516

$4+140$

TOT. $\quad 83829$

12315

414

34
314
31
314
314
31
34
30
30
30
30
30
30
30
30
30
30
30
30
30
30
30
30
30
30
30
30
30
876
31

2
2
2
2
2
2
2
2
2
2
2
2
2
2
2
2
2
2
2
2
2
2
2
2
2
2
2
2
56
2

HURST FILTER CIVIC STREET
ROAII PLANI CENIER

HOUSE IUI븐

$\begin{array}{ll}60 & 2350 \\ 60 & 2350 \\ 60 & 2350 \\ 60 & 2350 \\ 60 & 2350 \\ 60 & 2350 \\ 60 & 2350 \\ 60 & 2350 \\ 60 & 2350 \\ 60 & 2350 \\ 60 & 2350 \\ 60 & 2350 \\ 60 & 2350 \\ 60 & 2350 \\ 60 & 2350 \\ 75 & 2350 \\ 75 & 2350 \\ 75 & 2350 \\ 75 & 2350 \\ 75 & 2350 \\ 75 & 2350 \\ 75 & 2350 \\ 75 & 2350 \\ 75 & 2350 \\ 75 & 2350 \\ 75 & 2350 \\ 75 & 2350 \\ 75 & 2350 \\ & \\ 1676 & 65800 \\ 67 & 2350 \\ & \end{array}$

270
270
270
270
270
270
270
270
270
270
270
270
270
270
270
270
270
270
270
270
270
270
270
270
270
270
270
270
7560
270

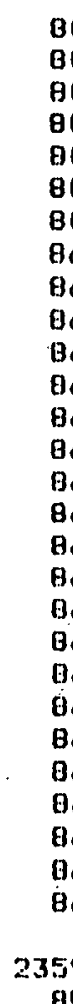

2443
24113

$352 \quad 7504$

$\begin{array}{lll}2443 & 352 & 7584 \\ 2443 & 352 & 7584\end{array}$

$2443 \quad 352 \quad 7504$

$2143 \quad 352 \quad 7584$

$2443 \quad 352 \quad 7584$

$2443 \quad 348$. 9609

$2143 \quad 3118 \quad 9656$

$24: 3 \quad 3116 \quad 9656$

$24143 \quad 349 \quad 9656$

$24+3 \quad 348 \quad 9656$

$2443 \quad 340 \quad 9656$

$2443 \quad 348 \quad 9656$

$2443 \quad 349 \quad 9656$

$2413 \quad 348 \quad 9671$

$2443 \quad 348 \quad 9671$

$2443 \quad 348$

$2443 \quad 3110 \quad 9671$

$\begin{array}{lll}24+3 & 3118 & 9671 \\ 2443 & 348 & 9671 .\end{array}$

$24+3 \quad 349 \quad 9671$

$24143 \quad 340 \quad 9671$

$21443 \quad 348 \quad 9671$

$21143 \quad 3110 \quad 9671$

$2443 \quad 3110 \quad 9671$

$2143 \quad 3118 \quad 2671$

$2443 \quad 3118.9671$

$55969400 \quad 9770 \quad 256076$

$2443 \quad 3119$

7116 
CITY OF WATERVLIET

TYPICAL POWEER CONEUTERTION FOR MARCH

\begin{tabular}{|c|c|c|c|c|c|c|c|c|c|c|c|c|c|c|c|c|}
\hline IIAYII & $\begin{array}{r}\text { ICE } \\
\text { RINUK }\end{array}$ & $\begin{array}{l}\text { SENIOR } \\
\text { CII.ARE }\end{array}$ & $\begin{array}{l}\text { CITY } \\
\text { HALLS }\end{array}$ & POOL-A & PQDL-E & $\begin{array}{l}\text { HULSON } \\
\text { SHIORES }\end{array}$ & $\begin{array}{r}\text { CITY } \\
\text { GARAGE }\end{array}$ & $\begin{array}{r}\text { GROTTO } \\
\text { COURI }\end{array}$ & $\begin{array}{l}\text { WI SWALL } \\
\text { AVENIJE }\end{array}$ & $\begin{array}{l}\text { PUIAPING } \\
\text { SIAIION }\end{array}$ & $\begin{array}{r}\text { HURST } \\
\text { RONLI }\end{array}$ & $\begin{array}{l}\text { FILTER } \\
\text { PLANI }\end{array}$ & $\begin{array}{r}\text { CIVIC } \\
\text { CENIER }\end{array}$ & $\begin{array}{l}\text { STREET } \\
\text { LIGUIS }\end{array}$ & $\begin{array}{r}\text { FIRE } \\
\text { LUUSEE }\end{array}$ & IOIAL \\
\hline 1 & 3516 & $4+11$ & 59 & 0 & 12 & 0 & 30 & $\ddots$ & 75 & 2350 & 1 & 270 & 86 & 2443 & 3118 & 7671 \\
\hline 2 & 3516 & 4110 & 99 & 0 & 12 & 0 & 30 & 2 & 75 & 2350 & 1 & 270 & 196 & 2153 & $3+6$ & 9381 \\
\hline 3 & 3516 & 11.411 & 57 & 0 & 12 & ) & 30 & 2 & 75 & 2350 & 1 & 270 & A6 & 2153 & 340 & 9381 \\
\hline 4 & 3516 & 4.4 .11 & 59 & 0 & 12 & 0 & 30 & 2 & 75 & 2350 & 1 & 270 & 86 & 2153 & 3148 & 9381 \\
\hline 5 & 3516 & it+11 & 99 & 0 & 12 & 0 & 30 & 2 & 75 & 2350 & 1 & 270 & 86 & 2153 & 3140 & 9381 \\
\hline 6 & 3516 & Ifit 0 & 99 & 0 & 12 & 0 & 30 & 2 & 75 & 2350 & 1 & 270 & $86^{\circ}$ & 2153 & 348 & $938 \mathrm{i}$ \\
\hline 7 & 3516 & $4+1$ & 99 & 0 & 12 & 0 & 30 & 2 & 75 & 2350 & 1 & 270 & 86 & 2153 & $3+\theta$ & 8383 \\
\hline 日 & 3516 & 441 & 99 & 0 & 10 & 0 & 30 & 2 & 75 & 2350 & 1 & 270 & 86 & 2153 & 348 & 9301 \\
\hline 10 & 2453 & $4+1$ & 89 & 0 & 10 & 67 & 21 & 2 & 75 & 2350 & 1 & 305 & 80 & 2153 & 326 & 0.383 \\
\hline 11. & 2453 & $1+1+1$ & 71 & 0 & 10) & 67 & 21 & 2 & 75 & 2350 & 1 & 305 & 80. & 2153 & 326 & 0375 \\
\hline 12 & 2453 & itit. & 91 & 0 & 10 & 67 & 21 & 2 & 75 & 2350 & 1 & 305 & B0 & 2153 & 326 & 8375 \\
\hline 13 & 2453 & 44. & 81 & ( & 10 & 67 & 21. & 2 & 75 & 2350 & 1 & 305 & 60 & 2153 & 326 & 0375 \\
\hline 1.1 & 2453 & $1+1+1$ & 91. & 0 & 10 & 67 & 21 & 2 & 75 & 2350 & 1 & 305 & Bo & 2153 & 326 & 18375 \\
\hline 15 & 21.53 .3 & $1+4.1$ & 91 & 0 & 10 & 67 & 21. & 2 & 75 & 2350 & 1 & 305 & 00 & 2153 & 326 & 0375 \\
\hline 16 & $2 ! 533$ & $4+41$ & 91 & 0 & 10 & 67 & 21 & 2 & 75 & 2350 & 1 & 305 & 日0) & 2153 & 326 & 8375 \\
\hline 17 & 2453 & $1+4.1$ & 91 & 0 & 10 & 67 & 21 & 2 & 75 & 2350 & 1. & 305 & 80 & 2153 & $3: 6$ & 4375 \\
\hline 18 & 21,53 & +41 & 91 & 0 & 10 & 67 & 21 & 2 & 75 & 2350 & 1 & 305 & B0 & 21.53 & 326 & 0375 \\
\hline 19 & 2453 & $1+41$ & 81 & 0 & 10 & 67 & 21 & 2 & 75 & 2350 & 1 & 305 & 80. & 2153 & 326 & 0375 \\
\hline 21. & 2458.3 & 44.1 & 91 & 0 & 10 & 67 & 21 & 2 & 71 & 2350 & 1 & 305 & 130 & 2153 & 326 & B371 \\
\hline 22 & 2453 & $1+41$ & 91 & 0 & 10 & 67 & 21 & 2 & 71 & 2350 & 1 & 305 & $\theta 0$ & 2153 & 326 & 0371. \\
\hline 23 & 2453 & $1+4.1$. & 71 & 0 & 10 & 67 & 21 & 2 & 71 & 2350 & 1. & 305 & 80 & 2153 & 326 & 8371 \\
\hline 24 & 2453 & 44.1 & 91. & 0 & 10 & 67 & 21. & 2 & 71 & 2350 & 1 & 305 & BO & 2153 & 326 & 0371 \\
\hline 25 & 2453 & $1+41$ & 91 & 0 & 10 & 67 & 21 & 2 & 71 & 2350 & 1 & 305 & 00 & 2153 & 326 & 9371 \\
\hline 26 & 2453 & $1+41$ & 91 & 0 & 10 & 67 & 21 & 2 & 71 & 2350 & 1 & 305 & BO & 2153 & 326 & 0371 \\
\hline 27 & $24+533$ & 441 & 91 & () & 10 & 67 & 21 & 2 & 71 & 2350 & 1 & 305 & $\theta 0$ & 2153 & 326 & 8371 \\
\hline 20 & 2453 & $1+41$ & 91 & 0 & 10 & 67 & 21 & 2 & 71 & 2350 & 1 & 305 & 80 & 2153 & 326 & 0371 \\
\hline 29 & 2453 & $1+111$ & 91. & 0 & 10 & 67 & 21 & 2 & 71 & 2350 & 1 & 305 & BO & 2153 & 326 & 0371 \\
\hline 30 & 2453 & 141 & 91 & 0 & 10 & 67 & 21 & 2 & 71 & 2350 & 1 & 305 & 00 & 2153 & 326 & 0371 \\
\hline 31. & 2453 & 441 & 91 & 0 & 10 & 67 & 21 & 2 & 71 & 2350 & 1 & 305 & 80 & 1992 & 326 & 1420? \\
\hline TOT. & 65599 & 136613 & 2901 & 0 & 323 & 146,5 & 741 & 62 & 2271 & 72950 & 16 & 9144 & 25.36 & 660185 & 11310 & 28076\% \\
\hline AVis. & 2761 & 1,41 & 211 & 0 & 10 & 47 & 24 & 2 & 73 & 2350 & 1 & 295 & $\theta 2$ & 2158 & 333 & Q 6571 \\
\hline
\end{tabular}


CITY OF WATERVLIE

IYOICAL POWEER CONSUTPPTION FOR APRIL

\begin{tabular}{|c|c|c|c|c|c|c|c|c|c|c|c|c|c|c|c|c|}
\hline IIAYH & $\begin{array}{l}\text { ICE } \\
\text { RINKK }\end{array}$ & $\begin{array}{l}\text { GENTOR } \\
\text { GI ARE }\end{array}$ & $\begin{array}{l}\text { CITY } \\
\text { HALL. }\end{array}$ & POOL $-A$ & PODL $-\underline{B}$ & $\begin{array}{l}\text { HUIISON } \\
\text { SHORES }\end{array}$ & $\begin{array}{r}\text { CITY } \\
\text { GARAGE }\end{array}$ & $\begin{array}{r}\text { GROTTO } \\
\text { COULI }\end{array}$ & $\begin{array}{l}\text { WI SWALLL } \\
\text { AVENIEE }\end{array}$ & $\begin{array}{l}\text { PUAPING } \\
\text { EIATION }\end{array}$ & $\begin{array}{r}\text { HIIRST } \\
\text { ROAI! }\end{array}$ & $\begin{array}{l}\text { F IL.TER } \\
\text { PLANI }\end{array}$ & $\begin{array}{r}\text { CIVIC } \\
\text { CENIER }\end{array}$ & $\begin{array}{l}\text { S TREET } \\
\text { LIGHIS }\end{array}$ & $\begin{array}{r}\text { FIRE: } \\
\text { HGUSEE }\end{array}$ & IOIAL \\
\hline 1 & 2453 & $4+1$ & 91 & 0 & 10 & 67 & 21 & 2 & 71. & 2350 & 1 & 305 & 81) & $199 ?$ & 326 & 8209 \\
\hline 2 & 21453 & $1+141$ & 91 & 0 & 10 & 67 & 21 & 2 & 71. & 2350 & 1 & 305 & 80 & 1992 & 326 & 0209 \\
\hline 4 & 2453 & 1141 & 91 & 0 & 10 & 67 & 21 & 2 & 71 & 2350 & 1 & 305 & Bo & 1992 & $3: 6$ & n200 \\
\hline 5 & 2453 & $1,4.1$ & 91. & 0 & 10 & 67 & 21. & 2 & 71 & 3350 & 1 & 305 & 80 & 1892 & $3: 6$ & BOOS \\
\hline 6 & 2453 & $1+42$ & 91 & 0 & 10 & 67 & 21 & 2 & 71 & 2350 & 1 & 305 & 80 & 1992 & 326 & 0210 \\
\hline 7 & 2453 & $4+42$ & 71 & 0 & 10 & 67 & 21 & 2 & 71 & 2350 & 1 & 305 & 80 & 1992 & 326 & 0210 \\
\hline$\theta$ & 2453 & 442 & 91 & 0 & 10 & 67 & 21 & 2 & 71 & 2350 & 1 & 305 & Bo & 1992 & 326 & 0210 \\
\hline 10 & 2453 & $1+42$ & 91 & 0 & 10 & 67 & 21 & 2 & 71 & 2350 & 1 & 305 & BO & 1992 & 326 & 8210 \\
\hline 11 & II. & $4+2$ & 91 & 0 & 98 & 67 & 12 & 2 & 71 & 2350 & 1 & 305 & 67 & 1992 & 240 & 5738 \\
\hline 12 & 0 & 442 & 103 & 0 & 98 & 67 & 12 & 2 & 71 & 2350 & 1 & 305 & 67 & 1992 & $2+0$ & 5750 \\
\hline 13 & 0 & 4.42 & 1.03 & 0 & 98 & 67 & 12 & 2 & 71. & 2350 & 1 & 305 & 67 & 1972 & 0 & 5750 \\
\hline 1.4 & 0 & 442 & 103 & 0 & $99^{\circ}$ & 67 & 12 & 2 & 71 & 2350 & 1 & 305 & 67 & 1992 & 2010 & 5750 \\
\hline 15 & 0 & 4142 & 103 & 0 & 98 & 67 & 12 & 2 & 71 & 2350 & 1 & 305 & 67 & 1992 & 2110 & 5750 \\
\hline 16 & 0 & $4+2$ & 103 & 0 & 98 & 67 & 12 & 2 & 71 & 2350 & 1 & 305 & 67 & 1992 & 241 & 5750 \\
\hline 17 & 0 & $1+42$ & 103 & 0 & 90 & 67 & 12 & 2 & 71. & 2350 & 1 & 305 & 67 & 1992 & 2140 & 5750 \\
\hline 18 & 0 & $4+2$ & 103 & 0 & 98 & 67 & 12 & 2 & 71 & 2350 & 1 & 305 & 67 & 1992 & 240 & 5750 \\
\hline 19 & 0 & 4.42 & 103 & 0 & 98 & 67 & 12 & 2 & 47 & 2350 & 1 & 3115 & 67 & 1992 & 240 & 5726 \\
\hline 21 & () & 442 & 103 & 0 & 98 & 67 & 12 & 2 & 47 & 2350 & 1 & 305 & 67 & 1972 & $2+40$ & 5726 \\
\hline 22 & 0 & 4122 & 103 & 0 & 98 & 67 & 12 & 2 & 117 & 2350 & 1 & 305 & 67 & 1992 & 240 & 5736 \\
\hline 23 & 0 & 4142 & 103 & 0 & $9 \theta$ & 67 & 12 & 2 & 47. & 2.350 & 1 & 305 & 67 & 1992 & 240 & 5726 \\
\hline 24 & 0 & 442 & 103 & 0 & 98 & 67 & 12 & 2 & 147 & 2350 & 1 & 305 & 67 & 1992 & 240 & 5720 \\
\hline 25 & 0 & 442 & 103 & 0 & 90 & 67 & 12 & 2 & 47 & 2350 & 1 & 305 & 67 & 1992 & 240 & 5726 \\
\hline 26 & 0 & 11.42 & 103 & : & 90 & 67 & 12 & 2 & 117 & 2350 & 1 & 305 & 67 & 1992 & 240 & 5726 \\
\hline 27 & 0 & 442 & 103 & 0 & 98 & 67 & 12 & 2 & 47 & 2350 & 1 & 305 & 67 & 1992 & 240 & 5726 \\
\hline $2 \theta$ & 0 & 4112 & 103 & 0 & $9 \theta$ & 67 & 12 & 2 & $1+7$ & 2350 & 1 & 305 & 67 & 1992 & 240 & 5726 \\
\hline 29 & 0 & 1,142 & 103 & 0 & 96 & 67 & 12 & 2 & 47 & 2350 & 1 & 305 & 67 & 1992 & 2 & 5726 \\
\hline 30 & 0 & 442 & 10.3 & 0 & 98 & 67 & 12 & 2 & $1+7$ & 2350 & $i$ & 305 & 67 & 1624 & 240 & 53501 \\
\hline TOT. & 24525 & 1.3254 & 2960 & 0 & $20 s ?$ & 1990 & 448 & .60 & 1833 & 70500 & 1.5 & 9155 & 2151 & 59390 & 0063 & 196420 \\
\hline AVG. & 818 & 442 & 99 & 0 & 69 & 67 & 15 & 2 & 61 & 2350 & 1 & 305 & 72 & 1980 & 267 & 65849 \\
\hline
\end{tabular}




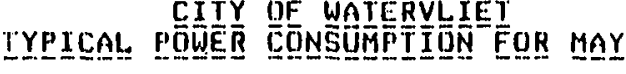

\begin{tabular}{|c|c|c|c|c|c|c|c|c|c|c|c|c|c|c|c|c|}
\hline IEYYI" & $\begin{array}{l}\text { ICE } \\
\text { RINKK }\end{array}$ & $\begin{array}{l}\text { SENTCOR } \\
\text { CIT.A\&E }\end{array}$ & $\begin{array}{l}\text { CITY } \\
\text { HALLي }\end{array}$ & POgL-A & PQDL-E & $\begin{array}{l}\text { HUDSON } \\
\text { SHURES }\end{array}$ & $\begin{array}{l}\text { CITY } \\
\text { GARAGE }\end{array}$ & $\begin{array}{r}\text { BROTTO } \\
\text { COURI }\end{array}$ & $\begin{array}{l}\text { WISWALL } \\
\text { AVENUE }\end{array}$ & $\begin{array}{l}\text { PUIAPING } \\
\text { SIAIIQN }\end{array}$ & $\begin{array}{r}\text { HURST } \\
\text { RQAI }\end{array}$ & $\begin{array}{r}\text { FIL.TER } \\
\text { PLANI }\end{array}$ & $\begin{array}{r}\text { CIVIC } \\
\text { CENIER }\end{array}$ & $\begin{array}{l}\text { STREET } \\
\text { LIGHIS }\end{array}$ & $\begin{array}{r}\text { FIRE } \\
\text { HOUSE }\end{array}$ & IOIAL \\
\hline 1 & 0 & 1422 & 103 & 0 & 98 & 67 & 12 & 2 & 47 & 2350 & 1 & 305 & 67 & 1624 & 2410 & $535 \theta$ \\
\hline 2 & 0 & 442 & 103 & 0 & 98 & .67 & 12 & 2 & 17 & 2350 & 1 & 305 & 67 & 1624 & $2+40$ & 5358 \\
\hline 3 & () & $1+42$ & 103 & 0 & 98 & 67 & 12 & 2 & 47 & 2350 & 1 & 305 & 67 & 1624 & 240 & $535 \theta$ \\
\hline 4 & () & $1+1+2$ & 103 & 0 & 90 & 67 & 12 & 2 & 47 & 2350 & 1 & 305 & 67 & 1624 & 240 & 5350 \\
\hline 5. & 0 . & $4+1$ & 103 & 0 & 91 & 67 & 12 & 2 & 47 & 2350 & 1 & 305 & 67 & 1624 & $2+0$ & 5357 \\
\hline 6 & 0 & $1+14.1$ & 103 & 0 & 913 & 67 & 12 & 2 & 47 & 2350 & 0 & 305 & 67 & 1624 & $2+0$ & 5357 \\
\hline 7. & 0 & $4+1$ & 103 & 0 & 98 & 67 & 12 & 2 & 47 & 2350 & 0 & 305 & 67 & 1624 & 20 & 5357 \\
\hline$B$ & 0) & $1+1+1$ & 103 & 0 & 90 & 67 & 12 & 2 & 47 & 2350 & 0 & 305 & 67 & 16211 & 0 & 5357 \\
\hline 9. & 0 & $1+1+1$ & 103 & 0 & 9.13 & 38 & 12 & 2 & $1+7$ & 2350 & 0 & 235 & 67 & 1624 & 240 & 5250 \\
\hline .1. 0 & 148 & $1+4.1$ & 103 & 1.3 & 9 & 38 & $q$ & 2 & 47 & 2350 & ) & 235 & 53 & 1624 & 152 & 5125 \\
\hline 11 & 118 & $41+1$ & .91 & 13 & 9 & 3日) & 9 & 2 & 47 & 2350 & 0 & 235 & 53 & 1624. & 152 & 5112 \\
\hline 12 & $1+8$ & +411 & 71 & 13 & 9 & 38 & 9 & 2 & 17 & 2350 & 0 & 235 & 53 & 1624 & 152 & 5112 \\
\hline 1.3 & $1+8$ & +41 & 91 & 13 & 9 & 38 & 9 & 2 & $1+7$ & 2350 & 0 & 235 & 53 & 1624 & 152 & 5112 \\
\hline 1.4 & $1+\theta$ & 441 & 71 & 1.3 & 9 & 30 & 7 & 2 & 47 & 2350 & 0 & 235 & 53 & 1624 & 152 & 5112 \\
\hline 15 & 148 & $1+41$ & 91 & 13 & 9 & 38 & 9 & 2 & 47 & 2350 & o & 235 & 53 & 1624 & 152 & 5112 \\
\hline 16 & 48 & $1+41$ & 91. & 13 & 9 & 36 & 9 & 2 & 47 & 2350 & 0 & 235 & 53 & 16211 & 152 & 5112 \\
\hline 17 & $1+8$ & .441 & 91 & 13 & 7 & 38 & 9 & 2 & 4.7 & 2350 & 0 & 235 & 53 & 1624 & 152 & 5112 \\
\hline 10 & 110 & $1+1$ & 91. & 13 & 9 & 39) & $?$ & 2 & 33 & 2350 & 0 & 235 & 53 & 1624 & $15 ?$ & $509 \theta$ \\
\hline 19 & 48 & 441 & 71 & 13 & 9 & 30 & 9 & 2 & 3.3 & 2350 & 0 & 235 & 5.3 & 1624 & 152 & 5098 \\
\hline $20 \%$ & $4 \theta$ & $1+11$ & 91 & 13 & 9 & 38 & 9 & 2 & 33 & 2350 & 0 & 235 & 53 & 1624 & 152 & 5098 \\
\hline 21 & 40 & $1+41$ & 9.1 & 13 & 9 & 38 & 9 & 2 & 33 & 2350 & 0 & 235 & 53 & 16,24 & 152 & 5090 \\
\hline 22 & 110 & 4.41 & 91 & 13 & 9 & 30 & 7 & 2 & 33 & 2350 & 0 & 235 & 53 & 1624 & 152 & 5098 \\
\hline 23 & 14.6. & $4+1$ & 91. & 1.3 & 9 & 38 & 9 & 2 & 33 & 2350 & 0 & 235 & 53 & 1624 & 152 & 5098 \\
\hline . & 48 & $\$ .4 .1$ & 91 & 1.3 & 9 & 38 & 9 & 2 & 33 & 2350 & 0 & 235 & 53 & 1624 & 152 & 5090 \\
\hline 25 & 48 & $4+1$ & 91 & 13 & 9 & 38 & 7 & 2 & 3.3 & 2350 & 0 & 235 & 53 & 1624 & 152 & 5090 \\
\hline 26 & 1,10 & 441 & 71 & 13 & 9 & 30 & 9 & 2 & 33 & 2350 & (). & 235 & 53 & 1624 & 152 & 5098 \\
\hline 27 & 1.9 & 1,41 & 91 & 13 & 9 & 38 & 9 & 2 & 3.3 & 2350 & 0 & 235 & 53 & 1624 & 152 & 5090 \\
\hline 28 & 118 & 441 & 91. & 1.3 & 9 & 38 & 9 & 2 & 33 & 2350 & $\dot{0}$ & 2.35 & 53 & 1624 & 152 & 5090 \\
\hline 29 & 14.6 & 441 & 91 & 13 & 9 & 38 & 9 & 2 & 33 & 2350 & 0 & 235 & 53 & 1624 & 152 & 5090 \\
\hline 30 & 149 & $14+1$ & 91 & 13 & 9 & 38 & 9 & 2 & 33 & 2350 & 0 & 235 & 53 & 1624 & 152 & 5090 \\
\hline 31 & 11.6 & $4+1$ & 91 & 13 & 9 & 39 & 9 & 2 & 33 & 2350 & 0 & 235 & 53 & 1414 & 152 & 49161 \\
\hline Tor . & 1. 05 & 1.3674 & $29+1$ & 292 & 1006 & 1.403 & 307 & 62 & 1248 & 72850 & 15 & 7848 & 1777 & 50169 & 5504 & 160221 \\
\hline AVG: & 34 & $14+1$ & 95 & 9 & 35 & 45 & 10 & 2 & 40 & 2350 & 0 & 253 & 57 & 1610 & 178 & 51.60 \\
\hline
\end{tabular}


CITY OF WATERVLIET

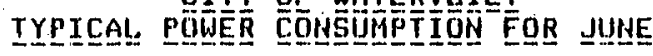

\begin{tabular}{|c|c|c|c|c|c|c|c|c|c|c|c|c|c|c|c|c|}
\hline "IAYY\| & $\begin{array}{r}\text { ICE } \\
\text { RIN! }\end{array}$ & $\begin{array}{r}\text { SENIOR } \\
\text { CIT.ARE }\end{array}$ & $\begin{array}{l}\text { CITY } \\
\text { HALLL }\end{array}$ & POQL-A & PQD黑- & $\begin{array}{l}\text { HUUSON } \\
\text { SHQRES }\end{array}$ & $\begin{array}{r}\text { CITY } \\
\text { GARAGE }\end{array}$ & $\begin{array}{r}\text { GROTTO } \\
\text { CDURI }\end{array}$ & $\begin{array}{l}\text { WISWALLL } \\
\text { AVENUE }\end{array}$ & $\begin{array}{l}\text { PUMPING } \\
\text { GTATION }\end{array}$ & $\begin{array}{r}\text { HURST } \\
\text { ROAII }\end{array}$ & $\begin{array}{l}\text { FILTER } \\
\text { PLANI }\end{array}$ & $\begin{array}{r}\text { CIVIC } \\
\text { CENTER }\end{array}$ & $\begin{array}{l}\text { STREET } \\
\text { LIGHIS }\end{array}$ & $\begin{array}{l}\text { FIRE } \\
\text { HOUSE: }\end{array}$ & TOTAL \\
\hline 1 & 48 & पil 1 & 91. & 13 & 9 & 38 & 9 & 2 & 33 & 2350 & 0 & 235 & 53 & 1444 & 152 & 4718 \\
\hline 2 & $1+6$ & $1+41$ & 7.1 & 13 & 9 & 39 & 9 & 2 & 33 & 2350 & 0 & 235 & 53 & $14+4$ & $15 ?$ & 4910 \\
\hline 3 & 40 & 㥩 & 9.1 & 13 & 9 & 38 & 9 & 2 & 33 & 2350 & 0 & 235 & 53 & $1 H H_{1}$ & 152 & 1918 \\
\hline if & 46 & $1,1+1$ & 91 & 13 & 9 & 38 & 9 & 2 & 33 & 2350 & 0 & 235 & 53 & 1444 & 152 & 1910 \\
\hline 5 & 190 & $\| \omega 1$ & 91 & 13 & 9 & 38 & 9 & 2 & 33 & 2350 & 0 & 235 & 53 & 14414 & 152 & 4910 \\
\hline 6 & 49 & 4.12 & 91. & 1.3 & 9 & 38 & 9 & 2 & 33 & 2350 & 0 & 235 & 53 & $14+4$ & 152 & 4919 \\
\hline 7 & $1+6$ & 442 & 91. & 13 & 9 & 38 & 9 & 2 & 33 & 2351 & () & 235 & 53 & 1444 & 152 & 4919 \\
\hline$\theta$ & +13 & 4.22 & 91. & 13 & 9 & 36 & 9 & 2 & .33 & 2350 & 0 & 235 & 53 & 14114 & 152 & 4919 \\
\hline 9 & 70 & 442 & 91 & - 181 & 5 & 30 & 9 & 2 & 3.3 & 2350 & 0 & 235 & $3 \theta$ & 1444 & 150 & 51195 \\
\hline 1. 0 & 7.13 & 1142 & 110 & 191 & 5 & 30 & 9 & 2 & 33 & 2350 & 0 & 235 & 36 & 1144 & 150 & 5114 \\
\hline 1.1 & 70 & 1142 & 110 & 181 & 5 & 38 & 9 & 2 & 33 & 2350 & 0 & 235 & 38 & 1444 & 150 & 5114 \\
\hline 12 & 76 & $1+1+2$ & 110 & 191 & 5 & 30 & 9 & 2 & 33 & 2350 & () & 235 & 38 & 14144 & 150 & 5114 \\
\hline 13 & 70 & $11+2$ & 110 & 181 & 5 & 38 & 9 & 2 & 33 & 2350 & 0 & 235 & 38 & 1444 & 150 & 5114 \\
\hline 14 & 79 & 4.42 & 110. & 101 & 5. & 38 & 9 & 2 & 33 & 2350 & 0 & 235 & 313 & 141,4 & 150 & 5114 \\
\hline 15 & 70 & $1+42$ & 110 & 181 & 5 & 38 & 7 & 2 & 33 & 2350 & 0 & 235 & 30 & 1444 & 151) & 5114 \\
\hline 16 & 78 & $4+2$ & 110 & 181 & 5 & 38 & 9 & 2 & 33 & 2350 & 0 & 235 & 38 & 14411 & 150 & 5114 \\
\hline 1.7 & $7 \theta$ & $1+1,2$ & 110 & 181 & 5 & 30 & 9 & 2 & 33 & 2350 & 0 & 235 & 38 & $14+14$ & 150 & 5114 \\
\hline 10 & $7 B$ & 442 & 110. & 181 & $\dot{i j}$ & 30 & 9 & 2 & 33 & 2350 & 0 & 235 & 38 & 14144 & 150 & 5114 \\
\hline 19 & .78 & $4+2$ & 110 & 181 & 5 & 38 & 9 & 2 & $1+4$ & 2350 & 0 & 235 & 38 & $14+44$ & 150 & 5125 \\
\hline 20 & 79 & $1+42$ & 110 & 181. & 5 & 38 & 9 & 2 & 4.4 & 2350 & 0 & 235 & 31 & 1444 & 150 & 5125 \\
\hline 21 & 78 & $4+2$ & 110 & 181 & 5 & 30 & 9 & 2 & 44 & 2350 & 0 & 235 & $3 \theta$ & $14+14$ & 151 & 5125 \\
\hline 22 & $7 \theta$ & $4+2$ & 110 & 181 & 5 & 30 & 9 & 2 & 44 & 2350 & 0 & 235 & 38 & 14144 & 150 & 5125 \\
\hline 23 & 70 & $4+2$ & 11.0 & 181 & 5 & 30 & 9 & 2 & 1,4 & 2351 & 0 & 235 & $3 \theta$ & $1+1,4$ & 150 & 5125 \\
\hline 24 & 73 & 1442 & 110 & $1 B 1$ & 5 & $3 \theta$ & 9 & 2 & 44 & 2350 & 0 & 235 & 38 & 14144 & 150 & 51.25 \\
\hline 25 & 78 & $41_{2} 2$ & 11.0 & 181 & 5 & 38 & 9 & 2 & 144 & 2350 & 0 & 235 & 30 & 1444 & 150 & 5125 \\
\hline 26 & 78 & $4+2$ & 110 & 181 & 5 & 30 & 9 & 2 & 1,14 & 2350 & 0 & 235 & $3 \theta$ & 14411 & 150 & 5125 \\
\hline 27 & $7 \theta$ & $1+1+2$ & 110 & 181 & 5 & $3 \theta$ & 9 & 2 & 44 & 2350 & 0 & 235 & 38 & 14114 & 151 & 51.25 \\
\hline 20 & 70 & 4.42 & 110 & 181 & 5 & 38 & 9 & 2 & 44 & 2350 & il & 235 & 30 & 1444 & 150 & 5125 \\
\hline 29 & 78 & 442 & 11.0 & 181 & 5 & 36 & 9 & 2 & 1,14 & 2350 & 0 & 235 & 38 & 1444 & 150 & 5125 \\
\hline 30 & 78 & $1+4.2$ & 1.10 & 181 & 5 & 39 & 9 & 2 & $41 \%$ & 2350 & 10 & 235 & 38 & 1250 & 150 & 4931 \\
\hline זיזנו & 2106 & 13256 & 3124 & 41179 & 174 & 1135 & 264 & 60 & 1113 & 70500 & 14 & 7052 & 1251 & 13131 & 15.16 & 151725 \\
\hline AVL. & 70 & $4+22$ & 104 & 136 & 6 & $3 \mathbf{3}$ & 9 & 2 & 37 & 2350 & 0 & 235 & 12 & 1.438 & 151 & 5059 \\
\hline
\end{tabular}


CITY QF WATERVLIET

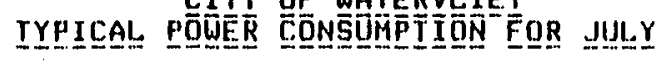

\begin{tabular}{|c|c|c|c|c|c|c|c|c|c|c|c|c|c|c|c|c|}
\hline lingi" & $\begin{array}{r}\text { ICE } \\
\text { RIVI! }\end{array}$ & $\begin{array}{r}\text { SENIOR } \\
\text { CIT.AKB }\end{array}$ & $\begin{array}{l}\text { CI TY } \\
\text { HALLL }\end{array}$ & $\underline{P O Q L}-\underline{A}$ & POQL-E & $\begin{array}{l}\text { HUISON } \\
\text { SHQORES }\end{array}$ & $\begin{array}{r}\text { CITY } \\
\text { GARAGE }\end{array}$ & $\begin{array}{r}\text { GROTTO } \\
\text { COURI }\end{array}$ & $\begin{array}{l}\text { WISWALL } \\
\text { AVENIJE }\end{array}$ & $\begin{array}{l}\text { PUMPING } \\
\text { SIATIOH }\end{array}$ & $\begin{array}{r}\text { HURST } \\
\text { ROAEI }\end{array}$ & $\begin{array}{r}\text { FILTER } \\
\text { PLANT }\end{array}$ & $\begin{array}{r}\text { CIVIC } \\
\text { CENIER }\end{array}$ & $\begin{array}{l}\text { STREET } \\
\text { LIGHISS }\end{array}$ & $\begin{array}{r}\text { FIRE } \\
\text { IIIUSE }\end{array}$ & Iula! \\
\hline 1 & 78 & $4+2$ & 110 & 131 & 5 & 38 & 9 & 2 & 44 & 2350 & 0 & 2.35 & 30 & 1250 & 150 & 493.1 \\
\hline 2 & 78 & $4+2$ & 110 & 101 & 5 & 38 & 9 & 2 & $1,1 / 4$ & 2350 & 1) & 235 & 38 & 1250 & 150 & 4931 \\
\hline 3 & 78 & 1412 & 110 & 181. & 5 & 38 & 9 & 2 & 4.4 & 2350 & 0) & 235 & 38 & 1250 & 150 & 4931 \\
\hline It & 78 & 442 & 110 & 181 & 5 & 38 & 9 & 2 & 4.4 & 2350 & 0 & 235 & 38 & 1250 & 150 & 4931 \\
\hline 5 & $7 B$ & 142 & 110 & 181 & 5 & 39 & 9 & 2 & 44 & 2350 & 0 & 235 & 38 & 1250 & 150 & 4931 \\
\hline 6 & 70 & 443 & 110 & 181 & 5 & 38 & 9 & 2 & 144 & 2350 & 0 & 235 & 30 & 1250 & 150 & 4933 \\
\hline 7 & 78 & 443 & 110 & 181 & 5 & 38 & 9 & 2 & 4.4 & 2350 & 0 & 235 & 39 & 1250 & 150 & 4932 \\
\hline$\theta$ & 78 & $4+3$ & 110 & $1 B 1$ & 5 & 38 & 9 & 2 & 4.14 & 2350 & 0 & 235 & 30 & 1250 & 150 & 4935 \\
\hline 9 & $7 \mathrm{G}$ & $4+3$ & $1 \mathrm{~d} .0$. & 181 & 5 & 40 & 9 & .2 & 44 & 2350 & 0 & 212 & 39 & 1250 & 150 & 4911 \\
\hline 10 & 78 & 443 & 110 & 181 & 5 & 40 & 9 & 2 & 4,4 & 2350 & 0 & 212 & 38 & 1250 & 150 & 4911 \\
\hline 11 & 112 & $1+43$ & 110 & 1.91 & 52 & 4.0 & $\theta$ & 2 & 44 & 2350 & 0 & 212 & 44 & 1250 & $16 \%$ & 504.1 \\
\hline 12 & 112 & 443 & 173 & 191 & 52 & 40 & 9 & 2 & 44 & 2350 & 0 & 212 & 144 & .1250 & 182 & 5104 \\
\hline 13 & 112 & $4 ! 13$ & 173 & 191 & 52 & 40 & 9 & 2 & 44 & 2350 & 0 & 212 & 44 & 1250 & 102 & 5104 \\
\hline 1.4 & 112 & $1+1+3$ & 173 & 191 & 52 & 40 & 9 & 2 & 1,4 & 2350 & 0 & 212 & 414 & 1250 & 182 & 5104 \\
\hline 15 & 112 & $41+3$ & 173 & 191 & 52 & 4.0 & 9 & 2 & 44 & 2350 & 0 & 212 & 44 & 1250 & 192 & 51114 \\
\hline 16 & 112 & $4+3$ & .173 & 191. & 52 & 40 & 9 & 2 & 1,4 & 2350 & 0 & 212 & 44 & 1250 & 162 & 5104 \\
\hline 17 & 112 & 1,443 & 173 & 191 & 52 & 1.0 & 9 & 2 & 44 & 2350 & 0 & 212 & 414 & 1250 & 182 & 51.01 \\
\hline 1.8 & 112 & 443 & 173 & 191 & 52 & 40 & 9 & 2 & 44 & 2350 & 0) & 212 & $1+4$ & 1250 & $19 ?$ & 5104 \\
\hline 19 & 112 & $1,4,3$ & 173 & 1.91 & 52 & 40 & 9 & 2 & $1+0$ & 2350 & 0 & 212 & 44 & 1250 & 102 & 5110 \\
\hline 20 & 1.22 & 44.3 & 173 & 191. & 52 & 40 & 9 & 2 & 40 & 2350 & 0 & 212 & 414 & 1250 & $1 \mathrm{BZ}$ & 5100 \\
\hline 2.1 & 112 & 1443 & 173 & 191 & 52 & .40 & 9 & 2 & 40 & 2350 & 0 & 212 & 44 & 1250 & 182 & 51100 \\
\hline 22 & 112 & 44.3 & 173 & 191 & 52 & 40 & 9 & 2 & 40 & 2350 & 0 & 212 & 114 & 1250 & 102 & 5100 \\
\hline 23 & 112 & $1+143$ & 173 & 191 & 52 & 40 & 7 & 2 & 40 & 2350 & 0 & 212 & 44 & 1250 & 182 & 5100 \\
\hline 24 & 112 & $4+43$ & 173 & 191 & 52 & 40 & 9 & 2 & 10 & 2350 & 0 & 212 & 114 & 1250 & 182 & 5100 \\
\hline 25 & 112 & $1+43$ & 173 & 191 & 52 & 40 & 9 & 2 & $4 \pi$ & 2350 & 0 & 212 & 44 & 1250 & $1 \mathrm{H2}$ & 5100 \\
\hline 26 & 112 & 1,43 & 173 & 191 & 52 & 40 & 9 & 2 & $1+0$ & 2350 & 0 & 212 & 44 & 1250 & 182 & 5100 \\
\hline 27 & 112 & 443 & 173 & 191 & 52 & 40 & 9 & 2 & 40 & 2350 & 0 & 212 & 1,4 & 1250 & 182 & 5100 \\
\hline $2 \beta$ & 112 & $4+3$ & 173 & 191 & 52 & 40 & 9 & 2 & 40 & 2350 & 0 & 212 & 11.4 & 1250 & 182 & 5100 \\
\hline 29 & 112 & $1+143$ & 173 & 191 & 52 & 40 & 9 & 2 & 40 & 2350 & 0 & 212 & 44 & 1250 & 102 & 51110 \\
\hline 30 & 112 & $1,4.3$ & 173 & 191 & 52 & 40 & 9 & 2 & 40 & 2350 & i) & 212 & 411 & 1250 & 182 & 5100 \\
\hline 31. & 112 & .443 & 173 & 191 & 52 & 411 & 9 & 2 & 40 & 2350 & 11 & 212. & 44 & 1342 & 102 & 5192 \\
\hline $\begin{array}{l}\text { TOT: } \\
\text { AVB: }\end{array}$ & $\begin{array}{r}3129 \\
101\end{array}$ & $\begin{array}{r}13737 \\
1+43\end{array}$ & $\begin{array}{r}4668 \\
151\end{array}$ & $\begin{array}{r}5813 \\
188\end{array}$ & $\begin{array}{r}1129 \\
36\end{array}$ & $\begin{array}{r}1231 \\
40\end{array}$ & 279 & 62 & $\begin{array}{r}1313 \\
4.2\end{array}$ & $\begin{array}{r}72850 \\
2350\end{array}$ & $\begin{array}{r}15 \\
11\end{array}$ & $\begin{array}{r}6746 \\
210\end{array}$ & $\begin{array}{r}1.296 \\
42\end{array}$ & $\begin{array}{r}30947 \\
1253\end{array}$ & $\begin{array}{r}5323 \\
172\end{array}$ & $\begin{array}{r}1561139 \\
51116\end{array}$ \\
\hline
\end{tabular}


CITY QF WATERVLIET

IYPICAL POWWER CONGUTPTIOTN FOR AUGUST

\begin{tabular}{|c|c|c|c|c|c|c|c|c|c|c|c|c|c|c|c|c|}
\hline IIAYH. & $\begin{array}{l}\text { ICE } \\
\text { RIIIIK }\end{array}$ & $\begin{array}{r}\text { SENIOR } \\
\text { CIT.ARE }\end{array}$ & $\begin{array}{l}\text { CITY } \\
\text { HALLL }\end{array}$ & PQDL-A & PQDLL $-\underline{B}$ & $\begin{array}{l}\text { HULISON } \\
\text { SHORES }\end{array}$ & $\begin{array}{r}\text { CITY } \\
\text { SARAGE }\end{array}$ & $\begin{array}{l}\text { GROTTO } \\
\text { COURI }\end{array}$ & $\begin{array}{l}\text { WI SWAL.L } \\
\text { AVENIJE }\end{array}$ & $\begin{array}{l}\text { PUMPING } \\
\text { STAIION }\end{array}$ & $\begin{array}{r}\text { HURST } \\
\text { ROAII }\end{array}$ & $\begin{array}{r}\text { FILTER } \\
\text { PLANI }\end{array}$ & $\begin{array}{r}\text { CIVIC } \\
\text { CENTER }\end{array}$ & $\begin{array}{l}\text { STREET } \\
\text { LIGHIS }\end{array}$ & $\begin{array}{r}\text { FI RE } \\
\text { HOUSE }\end{array}$ & TOTAL \\
\hline 1. & 112 & $4+43$ & 173 & 191 & 52 & 40 & 9 & 2 & 40 & 2350 & 0 & 212 & 414 & 1342 & 182 & 5172 \\
\hline 2 & 112 & $1+4.3$ & 173 & 191 & 52 & 10 & 9 & 2 & $1+0$ & 2350 & 0 & 212 & 44 & 1342 & 382 & 5192 \\
\hline 4 & 112 & 1,43 & 173 & 191 & 52 & $1+0$ & 9 & 2 & +11 & 2350 & 0 & 212 & $4 ! 4$ & $13+2$ & 182 & 5192 \\
\hline 5 & 112 & 1143 & 173 & 191 & 52 & $1+0$ & 9 & 2 & 40 & 2350 & 0 & 212 & 414 & 1342 & 182 & 5192 \\
\hline 6 & 112 & $1+1.3$ & 173 & 191 & 52 & 40 & 7 & 2 & 40 & 2350 & 0 & 212 & 44 & 1342 & 182 & 5192 \\
\hline 7 & 1.12 & $1+43$ & 173 & 191 & 52 & 40 & 9 & 2 & 40 & 2350 & 0 & 212 & 44 & 1342 & 102 & 5192 \\
\hline 日 & 112. & $1+1,3$ & 173 & 191 & 52 & 40 & 9 & 2 & 110 & 2350 & 0 & 212 & 44 & 13112 & 102 & 5192 \\
\hline 10 & 99 & 1143. & 157 & 91 & 70 & 40 & 10 & 2 & $1+0$ & 2350 & 0 & 212 & 34 & 1342 & 204 & 5095 \\
\hline 11 & 9.7 & 443 & 157 & 71 & 70 & 4.0 & 10 & 2 & 40 & 2350 & () & 212 & 34 & 1342 & 204 & 5095 \\
\hline 12 & 99 & 443 & 157 & 91 & 70 & 1,0 & 10 & 2 & 10 & 2350 & 0 & 212 & 34 & 1312 & 204 & 5095 \\
\hline 13 & 79 & $1,1.3$ & 157 & 91 & 70 & 140 & 10 & 2 & 40 & 2350 & 0 & 212 & 314 & 1342 & 204 & 5095 \\
\hline 14 & 99 & 44.3 & 157 & 91 & 70 & 40 & 10 & 2 & 40 & 2350 & 0 & 212 & 34 & 13112 & 204 & 51195 \\
\hline 15 & 99. & 44.3 & 157 & 91 & 70 & 110 & 10 & 2 & 40 & 2350 & 0 & 212 & 34 & 13112 & 204 & 5095 \\
\hline 1.6 & 99 & 443 & 157 & 91 & 70 & 10 & 10 & 2 & 40 & 2350 & 0 & 212 & 34 & 1342 & 204 & 51195 \\
\hline 17 & 99 & 443 & 157 & 91 & 70 & 40 & 10 & 2 & 35 & 2350 & 0 & 212 & 34 & 1342 & 204 & 5090 \\
\hline 1.9 & 99 & 443 & 157 & 91 & 70 & 40 & 10 & 2 & 35 & 2350 & 0 & 212 & 314 & 1342 & 204 & 5090 \\
\hline 19 & 99 & 1,43 & 157 & 91 & 70 & 40 & 10 & 2 & 35 & 2350 & 0 & 212 & 34 & 1342 & 204 & 5090 \\
\hline 21 & 99 & $1+43$ & 157 & 91 & 711 & 40 & 10 & 2 & 35 & 2350 & 0 & 212 & 314 & 1342 & 204 & 5090 \\
\hline 27 & 99 & $1+13$ & $15 \%$ & 91 & 70 & 40 & 10 & 2 & 35 & 2350 & 0 & 212 & 34 & 1342 & 204 & 5090 \\
\hline 23 & 99 & $1+43$ & 157 & 91 & 71 & 40 & 10 & 2 & 35 & 2350 & 0 & 212 & 34 & 1342 & 204 & 5090 \\
\hline 24 & 99 & $1+43$ & 157. & 91 & 70 & 40 & 10 & 2 & 35 & 2350 & 0 & 212 & 34 & 1342 & 2014 & 5090 \\
\hline 25 & 99 & 443 & 157 & 91 & 70 & 40 & 10 & 2 & 35 & 2350 & 0 & 212 & 34 & 1342 & 204 & 5090 \\
\hline 26. & 99 & $1+43$ & 157 & 91 & 70 & 40 & 10 & 2 & 35 & 2350 & 0 & 212 & 34 & 13142 & 204 & 5090 \\
\hline 27 & 99 & 41,3 & 157 & 91 & 70 & 110 & 10 & 2 & 35 & 2350 & 0 & 212 & 34 & 13112 & 204 & 5090 \\
\hline 20 & 99 & 413 & 157 & 91 & 70 & 40 & 10 & 2 & 35 & 2350 & 0 & 212 & 34 & 1312 & 2014 & 5090 \\
\hline 29 & 99 & $1+1+3$ & 157 & 91 & 70 & $1+0$ & 10 & 2 & 35 & 2350 & 0 & 212 & 34 & 13112 & 204 & 5090 \\
\hline 311 & 99 & 4143 & 157 & 91 & 70 & 140) & 10 & 2 & 35 & 2350 & 0 & 21.2 & 34 & 13112 & 20.14 & 5070 \\
\hline 31 & 99 & 1,43 & 157 & 91 & 70 & 40 & 10 & 2 & 35 & 2350 & i) & 212 & 34 & 1501 & 2014 & 5329 \\
\hline Tor. & $31 \mathrm{~s}^{4}$ & 13735 & 5015 & 3623 & 2029 & 1252 & 303 & 62 & 1177 & 72050 & 15 & 6,557 & 1133 & 110.31 & 6119 & 1 С日E) \\
\hline AVG. & 1.02 & 143 & 162 & 117 & 65 & 110 & 1. 0 & 2 & 30 & 2350 & 0 & 212 & 37 & 13119 & 190 & 5126 \\
\hline
\end{tabular}


CITY OF WATERVLIET

IYPICAL POWER CONEUMPTION EOR SEPTEMEER

IAYY




1
1
1
1
1
1
1
1
1
1
20
2
2
2
2
2
2
2
2
2
3

ICE GENIOR RINK CII. ARI

(19

Tor.
CITY HALي는.

IIUTIGON

CITY GROTTO WISWALL. PUMPING SHORES GARAGE COURI AVENUE SIATION

OgL-A PQOLL

$\begin{array}{ll}157 & 91 \\ 157 & 91 \\ 157 & 91 \\ 157 & 71 \\ 157 & 91 \\ 157 & 91 \\ 157 & 91 \\ 157 & 0 \\ 100 & 0 \\ 100 & 0 \\ 100 & 0 \\ 100 & 0 \\ 100 & 0 \\ 100 & 0 \\ 100 & 0 \\ 100 & 0 \\ 100 & 0 \\ 100 & 0 \\ 100 & 0 \\ 101 & \\ 100 & 0 \\ 100 & 0 \\ 101 & 0 \\ 100 & 0 \\ 100 & 0 \\ 103 & 0 \\ 101 & 0 \\ 100 & 0 \\ 101 & 0 \\ 100 & 0 \\ & 0 \\ 3159 & 040\end{array}$

HI.JR BOAEL
FILTER C.IVIC STREET ELANI CENIER LIGHIS

FIRE HOUSEE IOINL

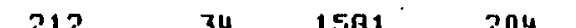

$\begin{array}{lllll}212 & 34 & 1501 & 204 & 5329\end{array}$ $\begin{array}{lllll}212 & 34 & 1581 & 204 & 5329\end{array}$ $\begin{array}{lllll}212 & 34 & 1581 & 204 & 5329\end{array}$ $\begin{array}{lllll}212 & 34 & 1581 & 204 & 5329\end{array}$ 212 31 $\begin{array}{lllll}212 & 34 & 1581 & 204 & 5329 \\ 212 & 67 & 1581 & 154 & 5223\end{array}$ $\begin{array}{lllll}212 & 67 & 1581 & 154 & 5166\end{array}$ $\begin{array}{lllll}518 & 67 & 1581 & 154 & 5461\end{array}$ $\begin{array}{lllll}518 & 67 & 1581 & 154 & 5461\end{array}$ $\begin{array}{lllll}518 & 67 & 1581 & 154 & 5461\end{array}$ $\begin{array}{lllll}518 & 67 & 1581 & 154 & 51161\end{array}$ $\begin{array}{lllll}518 & 67 & 1581 & 154 & 5461 \\ 518 & 67 & 1501 & 154 & 5461\end{array}$ $\begin{array}{lllll}518 & 67 & 1581 & 151 & 5461\end{array}$ $\begin{array}{lllll}518 & 67 & 1581 & 1514 & 51461\end{array}$ $\begin{array}{lllll}518 & 67 & 1581 & 154 & 5462\end{array}$ $\begin{array}{lllll}518 & 67 & 1501 & 154 & 5462\end{array}$ $\begin{array}{lllll}518 & 67 & 1581 & 154 & 5462\end{array}$ $\begin{array}{lllll}518 & 67 & 1581 & 154 & 5462 \\ 518 & 67 & 1581 & 154 & 5462\end{array}$ $\begin{array}{lllll}518 & 67 & 1581 & 154 & 5463\end{array}$ $\begin{array}{lllll}518 & 67 & 1501 & 1511 & 5462\end{array}$ $1591 \quad 154 \quad 54+62$ 518 67 1581 154 5462 $\begin{array}{lllll}518 & 67 & 1581 & 154 & 5462 \\ 518 & 67 & 1581 & 154 & 5467\end{array}$ $\begin{array}{lll}518 & 67 & 1501 \\ 518 & 67 & 154\end{array}$ $\begin{array}{lllll}518 & 67 & 1561 & 154 & 5462 \\ 518 & 67 & 1697 & 154 & 5579\end{array}$ $12785 \quad 1783 \quad 47537 \quad 1961162511$ 
CITY OF WATERVLIET

IYPICAL POWWER CODNSUMPTIION EOR QCIOHER

\begin{tabular}{|c|c|c|c|c|c|c|c|c|c|c|c|c|c|c|c|c|}
\hline IIAYU & $\begin{array}{l}\text { ICE } \\
\text { RIIN! }\end{array}$ & $\begin{array}{r}\text { SENIOR } \\
\text { CIT.ARE }\end{array}$ & $\begin{array}{l}\text { CITY } \\
\text { HALLL }\end{array}$ & PQDL-A & PQDL-B & $\begin{array}{l}\text { HULION } \\
\text { SHORES }\end{array}$ & $\begin{array}{r}\text { CITY } \\
\text { GARAGE }\end{array}$ & $\begin{array}{r}\text { GROT TO } \\
\text { COUQRI }\end{array}$ & $\begin{array}{l}\text { WISWALL } \\
\text { AVENUE }\end{array}$ & $\begin{array}{l}\text { PUMPING } \\
\text { SIAIION }\end{array}$ & $\begin{array}{r}\text { HURST } \\
\text { ROAL }\end{array}$ & $\begin{array}{r}\text { FILTER } \\
\text { PLANI }\end{array}$ & $\begin{array}{l}\text { CIVIC } \\
\text { CENIER }\end{array}$ & $\begin{array}{l}\text { STRE.ET } \\
\text { LIGHIS }\end{array}$ & $\begin{array}{l}\text { FIRE } \\
\text { HOUSE }\end{array}$ & IOIAL \\
\hline 1 & 79 & $4+43$ & 100 & 0 & 67 & 29 & 15 & 2 & 37 & 2350 & 0 & 510 & 67 & 1697 & 154 & 5579 \\
\hline 2 & 99 & 4.43 & 100 & 0 & 6.7 & 29 & 15 & 2 & 37 & 2350 & 0 & 518 & 67 & 1697 & 154 & 5579 \\
\hline 4 & 99 & 44.3 & 100 & 0 & 67 & 29 & 15 & 2 & 37 & 2350 & 0 & 518 & 67 & 1697 & 154 & 5578 \\
\hline 5 & 99 & 413 & 100 & 0 & 67 & 29 & 15 & 2 & 37. & 2350 & 0 & 518 & 67 & 1697 & 154 & 5578 \\
\hline 6 & 99 & 1143 & 100 & 0 & 67 & 29 & 15 & 2 & 37 & 2350 & 0 & 518 & 67 & 1697 & 154 & 5578 \\
\hline 7 & 97 & 1113 & 100 & 0 & 67 & 29 & 15 & 2 & 37 & 2350 & 0 & 518 & 74 & 1697 & 154 & 5585 \\
\hline$\theta$ & 9.9 & 14143 & 100 & 0 & 51 & 29 & 15 & 2 & 37 & 2350 & 0 & 516 & 80 & 1697 & 154 & 5575 \\
\hline 10 & 1372 & 443 & .94 & 0 & 5.1 & 29 & 17 & 2 & 37 & 2350 & (1) & 518 & 80 & 1697 & 113 & 6804 \\
\hline 11 & 1372 & 4113 & 94 & 0 & 51 & 29 & 17 & 2 & 37 & 2350 & 0 & 518 & go & 1697 & 113 & 6804 \\
\hline 12 & 1372 & $1+143$ & 914 & 0 & 51 & 29 & 17 & 2 & 37 & 2350 & 0 & 510 & 80 & 1697 & 113 & GEUNI \\
\hline 13 & 1372 & 1443 & 94 & 0 & 51 & 29 & 17 & 2 & 37 & 2350 & 0 & 510 & 80 & 1697 & 113 & 6004 \\
\hline 14 & 1372 & 1.43 & 94 & 0 & 51 & 29 & 17. & 2 & 37 & 2350 & 0 & 510 & BO & 1697 & 113 & 6004 \\
\hline 15 & 1372 & $1,1+3$ & 911 & 0 & 51 & 29 & 17 & 2 & 37 & 2350 & 0 & 518 & $\theta 0$ & 1697 & 113 & 6804 \\
\hline 16 & 1372 & $1+43$ & 94 & 0 & 51 & 29 & 17 & 2 & 37 & 2350 & 0 & 518 & 00 & 1697 & 113 & 60304 \\
\hline 17 & 1372 & $1+43$ & 914 & 0 & 51 & 29 & 17 & 2 & 37 & 2350 & 0 & 518 & BO & 1697 & 113 & 6804 \\
\hline 10 & 1372 & $4+3$ & 94 & 0 & 51 & 29 & 17 & 2 & 33 & 2350 & 0 & 518 & BO & 1697 & 113 & 6801 \\
\hline 19 & 1372 & $1+43$ & 94 & 0 & 51 & 29 & 17 & 2 & 33 & 2350 & 0 & 518 & Bo & 1697 & 113 & 6001 \\
\hline 21 & 1372 & 1,143 & 911 & 0 & 51 & 29 & 17 & 2 & 33 & 2350 & 0 & 518 & Bo & 1697 & 113 & 6801 \\
\hline 22 & 1372 & $41+3$ & $94^{\circ}$ & 0 & 5.1 & 29 & 17 & 2 & 33 & 2350 & 0 & 516 & $\theta 0$ & 1697 & 113 & 6801 \\
\hline 23 & 1372 & 1143 & 9.1 & 0 & 51 & 29 & 17 & 2 & 33 & 2350 & 0 & 518 & BO & 1697 & 113 & 6001 \\
\hline 214 & 1372 & $4+43$ & 914 & 0 & 51 & 29 & 17 & 2 & 33 & 2350 & 0 & 510 & Bo & 1697 & 113 & 6001 \\
\hline 25 & 1372 & $1+113$ & 911 & 0 & 51 & 29 & 17 & 2 & 33 & 2350 & 0 & 510 & 100 & 1697 & 113 & $680 \mathrm{j}$ \\
\hline 26 & 1372 & 443 & 94 & 0. & 51 & 29 & 17 & 2 & 33 & 2350 & 0 & 518 & $B 0$ & 1697 & 113 & 6001 \\
\hline 27 & 1372 & 4.11 .3 & 914 & 0 & 51 & 29 & 17 & 2 & 33 & 2350 & 0 & 510 & 80 & 1697 & 113 & 6ย्ड 1 \\
\hline 28 & 1372 & $1+1+3$ & 94 & 0 & 51 & 29 & 17 & 2 & 33 & 2350 & 0 & 516 & B0 & 1697 & 113 & 6001 \\
\hline 29 & 1372 & $4+3$ & 94 & 0 & 51 & 29 & 17 & 2 & 33 & 2350 & 0 & 518 & 00 & 1697 & 113 & 6801 \\
\hline 30 & 1372 & 4,43 & 94 & 0 & 51 & 29 & 17 & 2 & 33 & 2350 & 0 & 518 & 80 & 1697 & 113 & 6801 \\
\hline 31 & 1372 & $4+3$ & 914 & 0 & 51 & 29 & 17 & 2 & 33 & 2350 & $B$ & 518 & 00 & 2064 & 113 & 7167 \\
\hline ror. & 31001 & 13724 & 29.69 & 3 & 1694 & 909 & 513 & 62 & 10134 & 72050 & 15 & 161063 & 2403 & 52906 & 3866 & 200221 \\
\hline AVG. & 1003 & 443 & 96 & 0 & 55 & 29 & 17 & 2 & 35 & 2350 & 0 & 510 & 78 & 17119 & 125 & 61159 \\
\hline
\end{tabular}


IYPICAL POWTER OE WATERVLIET

\begin{tabular}{|c|c|c|c|c|c|c|c|c|c|c|c|c|c|c|c|c|}
\hline IIAYII & $\begin{array}{l}\text { ICE } \\
\text { RINKK }\end{array}$ & $\begin{array}{l}\text { SENIOR } \\
\text { EII.ARE }\end{array}$ & $\begin{array}{l}\text { CITY } \\
\text { HALLL }\end{array}$ & PQDL-A & PQDL - & $\begin{array}{l}\text { HUDSON } \\
\text { SHORES }\end{array}$ & $\begin{array}{r}\text { CITY } \\
\text { GARAGE }\end{array}$ & $\begin{array}{r}\text { GROTTO } \\
\text { COUKI }\end{array}$ & $\begin{array}{l}\text { HISWALLL } \\
\text { AVENUEE }\end{array}$ & $\begin{array}{l}\text { PUMF' ING } \\
\text { STAIIQN }\end{array}$ & $\begin{array}{r}\text { HURST } \\
\text { ROAI }\end{array}$ & $\begin{array}{l}\text { FILTER } \\
\text { PLANI }\end{array}$ & $\begin{array}{r}\text { CIVIC } \\
\text { CENIER }\end{array}$ & $\begin{array}{l}\text { STREET } \\
\text { LIGIISS }\end{array}$ & $\begin{array}{r}\text { FIRF } \\
\text { HOUSE }\end{array}$ & IUIAL \\
\hline 1 & 1372 & 11.43 & 914 & 0 & 51 & 29 & 17 & 2 & 33 & 2350 & 0 & 518 & 80 & 2064 & 113 & 7167 \\
\hline 2 & 1372 & 1412 & 914 & 0 & 51 & 29 & 17 & 2 & 33 & 2350 & 0 & 518 & 80 & 2064 & 113 & 7166 \\
\hline 4 & 1372 & $1+1+1$ & 94 & 0 & 51 & 27 & 17 & 2 & 33 & 2350 & 0 & 518 & BO & 2064 & 113 & 7166 \\
\hline 5 & 1372 & 441 & 94 & 0 & 51 & 29 & 17 & 2 & 33 & 2350 & 0 & 518 & . 90 & 2064 & 113 & 7160 \\
\hline 6 & 1372 & $\| 1$ & 914 & 0 & 51 & 29 & 17 & 2 & 33 & 2350 & 0 & 510 & 01) & 2064 & 113 & 7166 \\
\hline 7. & .1372 & 4111 & 914 & 0 & 30 & 06 & 17 & 2 & 33 & 2350 & 0 & 384 & BO & 2064 & 113 & 7060 \\
\hline$\theta$ & 1.032 & $1+1+1$ & 94 & () & 10 & 86 & 24 & 2 & 33 & 2350 & 0 & 384 & B1 & 2064 & 166 & 6768 \\
\hline 10 & 10,32 & औ11 & 94 & 0 & 10 & $1+3$ & 31 & 2 & 33 & 2350 & 0 & 250 & B1 & 2064 & 166 & 66913 \\
\hline 11 & 1032 & 4111 & 914 & 0 & 10 & .243 & 31 & 2 & 33 & 2.3500 & 0 & 250 & (日) & 2064 & 16,6 & 6698 \\
\hline 12 & 10.32 & 1441 & 914 & 0 & 10 & 14.3 & 31 & 2 & 33 & 2350 & 0 & 250 & 81 & 2064 & 16,6 & 6690 \\
\hline 1.3 & 1032 & $\| 11$ & 911 & 0 & 10 & 143 & 31 & 2 & 3.3 & 2350 & 0 & 250 & 01 & 2064 & 16,6 & 6696 \\
\hline 14 & 1032 & 44.1 & 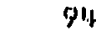 & 0 & 10 & 143 & 31 & 2 & 33 & 2350 & 0 & 250 & 81 & 2064 & 166 & 6698 \\
\hline 1.5 & 1032 & 441 & 94 & 0 & 10 & 143 & 31 & 2 & 33 & 2350 & 0 & 250 & 01 & 2064 & 166 & 6698 \\
\hline 16 & 1.032 & $4+1$ & 94 & 0 & 10 & 1,3 & 31. & 2 & 33 & 2350 & 0 & 250 & 81 & 20614 & 166 & 6690 \\
\hline 17 & $1.03 \%$ & $1+41$ & 914 & 0 & 10 & 143 & 31 & 2 & 40 & 2350 & 0 & 250 & E) 1 & 2064 & 16,6 & 6705 \\
\hline 10 & 10.32 & 4.41 & 94 & 0 & 1. (i) & 143 & 31 & 2 & 40 & 2350 & 0 & 250 & $\theta 1$ & 2064 & 192 & 6730 \\
\hline 18 & 1032 & 441 & 914 & 0 & 10 & 143 & 31 & 2 & 40 & 2350 & 0 & 250 & B1 & 2064 & 192 & 6730 \\
\hline 21 & 1032 & 141 & 94 & 0 & 10 & 143 & 31 & 2 & 1.10 & 2350 & 0 & 250 & B1 & 2064 & 192 & 6730 \\
\hline 22 & 1.032 & $1+41$ & 914 & 0 & 10 & 143 & 31 & 2 & 40 & 2350 & 0 & 250 & B1 & 2064 & 192. & 6730 \\
\hline 23 & 1032 & 141 & 94 & 0 & $10^{\circ}$ & 143 & 31 & 2 & 40 & 2350 & 0 & 250 & B1 & 2064 & 192 & 6730 \\
\hline 24 & 1032 & $4+1$ & 274 & 0 & 10 & 143 & 31 & 2 & 40 & 2350 & 0 & 250 & B1 & 2064 & $1 ? 2$ & 6730 \\
\hline 25 & 1.1032 & 4.41 & 914 & 0 & 10 & $1+3$ & 31. & 2 & 40 & 2350 & 0 & 250 & $\theta 1$ & 2064 & 192 & 6730 \\
\hline 26 & 1032 & $4+1$ & 94 & 0 & 1.1) & 143 & 31 & 2 & $1+0$ & 2350 & 0 & 250 & 81 & 206 't & 192 & 6730 \\
\hline 27 & 1.032 & 4111 & $9 i^{2}$ & 0 & 10 & 143 & 31 & 2 & $1+10$ & 2350 & 0 & 250 & 81. & 2064 & 192 & 6730 \\
\hline 20 & 1.032 & $4+1$ & 94. & 0 & 111 & 143 & 31. & 2 & 40 & 2350 & 0 & 250 & 81 & 2064 & 172 & 6730 \\
\hline 29 & 1032 & $.14+1$ & 914 & 0 & 10 & $1+3$ & 31 & 2 & 40 & 2350 & 0 & 250 & 81 & 2064 & 192 & 6730 \\
\hline 30 & 1032 & 4141 & 94 & 0 & 10 & 14.3 & 31 & 2 & 40 & 2350 & 0 & 250 & 81 & 2160 & 192 & 6827 \\
\hline TOT. & $.333+3$ & 13233 & 2824 & D & 560 & $31+4.4$ & B.16 & 59 & 1009 & 70500 & 14 & 9516 & $243 i$ & 62012 & 14944 & 2114785 \\
\hline$A \vee B ;$ & . 11:11 & $11+1$ & 94 & 0 & 19 & 115 & 27 & 2 & 36 & 2350 & 0 & 317 & A.1 & 2067 & 165 & 68126 \\
\hline
\end{tabular}


IYPICAL PŌWER EONGUMPIION EOR DECEMEER

\begin{tabular}{|c|c|c|c|c|c|c|c|c|c|c|c|c|c|c|c|c|}
\hline IIAYU & $\begin{array}{r}\text { ICE } \\
\text { R ITIN }\end{array}$ & $\begin{array}{r}\text { SENTOR } \\
\text { CET.ARE }\end{array}$ & $\begin{array}{l}\text { EITY } \\
\text { HALL }\end{array}$ & PDQL-A & PQDL $-\mathrm{B}$ & $\begin{array}{l}\text { HULISON } \\
\text { SHUORES }\end{array}$ & $\begin{array}{r}\text { CITY } \\
\text { GARAGE }\end{array}$ & $\begin{array}{r}\text { GROTTO } \\
\text { CD!URI }\end{array}$ & $\begin{array}{l}\text { WI SWALL } \\
\text { AVENIJE }\end{array}$ & $\begin{array}{l}\text { PUMFING } \\
\text { SIAIION }\end{array}$ & $\begin{array}{l}\text { HURST } \\
\text { ROAP }\end{array}$ & $\begin{array}{c}\text { F IL.TER } \\
\text { PLANI }\end{array}$ & $\begin{array}{r}\text { CIVIC } \\
\text { CENIER }\end{array}$ & $\begin{array}{l}\text { STREET } \\
\text { LIGUISS }\end{array}$ & $\begin{array}{r}\text { FIRE } \\
\text { HOUSSE }\end{array}$ & IOInL \\
\hline 1 & .1032 & $1+141$ & $9 i f$ & () & 10 & $1: 1+3$ & 31 & 2 & 40 & 2350 & 0 & 250 & B1 & 2160 & 192 & 6827 \\
\hline 2 & 1032 & $1+1+1$ & 914 & 0 & 1:0 & $1+3$ & 31 & 2 & 40 & 2350 & 0 & 250 & E1 & 2160 & $19 ?$ & 6927 \\
\hline 4 & 1032 & 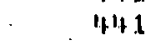 & 94. & 0 & 10 & $1+3$ & 31 & 2 & 40 & 2350 & 0 & 250 & $B 1$ & 2160 & 192 & 6027 \\
\hline 5 & 1032 & $1+4.1$ & $91 \mathrm{H}$ & 0 & 10 & 143 & 31 & 2 & 40 & 2350 & 0 & 250 & B1 & 2160 & 192 & 6827 \\
\hline 6 & 1032 & 438 & 94 & 0 & 10 & 143 & 31 & 2 & $1+0$ & 2350 & 0 & 250 & E1 & 2160 & $1 \% 2$ & 6824 \\
\hline 7 & 1032 & 439 & $9 t$ & 0 & 10 & 143 & 31 & 2 & $1+0$ & 2350 & 0 & 250 & $\theta 1$ & 2160 & 192 & 68214 \\
\hline 8 & 8607 & 1431 & 94 & 0 & 4.3 & 143 & 31 & 2 & $1+0$ & 2350 & 0 & 250 & 日1 & 2160 & 205 & 141,46 \\
\hline J. $\mathrm{A}$. & 8607 & $1+39$ & 91 & 0 & 4.3 & .143 & 31 & 2 & 40 & 2350 & 0 & 250 & 11 & 2160 & 205 & 141,43 \\
\hline 11 & 96,07 & 4.38 & 91 & 0 & 43 & 143 & 31 & 2 & 40 & 2350 & 0 & 250 & B1 & 2160 & 205 & 141443 \\
\hline 12 & 9607 & 1.38 & 91 & o & 43 & 14.3 & 31 & 2 & $4 n$ & 2350 & 0 & 250 & Q1 & 2160 & 205 & 141443 \\
\hline 13 & $136,17$. & $1+30$ & 91. & 0 & 4.3 & 143 & 31 & 2 & 40 & 2350 & 0 & 250 & 81 & 2160 & 205 & 14443 \\
\hline 1.4 & 0607 & 136 & 91 & 0 & 43 & 143 & 31 & 2 & it 0 & 2350 & 0 & 250 & 81 & 2160 & 205 & 144,43 \\
\hline 15 & 06107 & $1+38$ & 91. & 0 & 13 & 143 & 31 & 2 & 40 & 2350 & 0 & 250 & 01 & 2160 & 205 & $14+43$ \\
\hline 16 & .0607 & .438 & 91 & 0 & $1+3$ & 143 & 31. & 2 & 40 & 2350 & () & 250 & $\theta 1$ & 2160 & 205 & 14443 \\
\hline 17 & 8607 & 430 & 91 & 0 & 43 & 143 & 31 & 2 & 40 & 2350 & 0 & 250 & 81 & 2160 & 205 & 14443 \\
\hline 19 & 0607 & $1+38$ & 91 & 0 & 43 & 143 & 31. & 2 & 39 & 2350 & 0 & 250 & Bi & 2160 & 2117 & 14484 \\
\hline 19 & $\theta 6 ; 117$ & $1+30$ & 91 & 0 & 4.3 & 143 & 31 & 2 & 39 & 2350 & 0 & 250 & $\theta 1$ & 2160 & 247 & 141484 \\
\hline 21 & 18607 & $1+38$ & 9.1 & () & 143 & 143 & 31 & 2 & 39 & 2350 & 0 & 250 & B1 & 2160 & 247 & 141184 \\
\hline 22 & 0607 & 4.313 & 91. & 0 & 4.3 & $1+3$ & 31 & 2 & 39 & 2350 & 0 & 250 & 131 & 2160 & 247 & 14484 \\
\hline 23 & 06117 & $1+38$ & 91 & 0 & 43 & 143 & 31. & 2 & 34 & 2350 & 0 & 250 & 01 & 2160 & 247 & $1+1,64$ \\
\hline 24 & 8607 & $1+36$ & 91. & 0 & 13 & 143 & 31 & 2 & 39 & 2350 & 0 & 250 & 01 & 2160 & 247 & .14484 \\
\hline 25 & 06,07 & 430 & 91 & 0 & $1+3$ & 143 & 31 & 2 & 39 & 2350 & 0 & 250 & 81 & 2160 & $?$ & $141+814$ \\
\hline 26 & 0607 & 1.38 & 91 & 0 & 43 & 143 & 31 & 2 & 39 & 2350 & I) & 251 & $B 1$ & 2160 & 247 & IIIU⿴囗十 \\
\hline 27 & 8607 & 436 & 91 & 0 & $1+3$ & 143 & 31 & 2 & 39 & 2350 & 0 & 250 & 81 & 2160 & 247 & 14404 \\
\hline $2 \theta$ & 8607 & 4.38 & 91. & 0 & 43 & 11.43 & 31 & 2 & 39 & 2350 & 0 & 250 & Q1. & 2160 & 247 & 111484 \\
\hline 29 & 0607 & 438 & 91 & 0 & 4.3 & 143 & 31 & 2 & 39 & 2350 & 0 & 250 & $\theta 1$ & 2160 & 2117 & 14404 \\
\hline 30 & 01607 & $1+36$ & 91 & 0 & 43 & 143 & 31 & 2 & 39 & 2350 & 0 & 250 & 81 & 2160 & 247 & 14484 \\
\hline 31 & 86117 & $1+30$ & 91 & 0 & 4.3 & 143 & 31 & 2 & 39 & 2350 & 0 & 250 & 81 & $2431 t$ & 247 & 14757 \\
\hline TOT. & 213798 & 1.359 .3 & 2652 & 0 & 1106 & $41+12$ & 951 & 59 & 1232 & 72850 & 15 & 7757 & 2514 & 67240 & 6055 & $\begin{array}{r}395271 \\
12751\end{array}$ \\
\hline AVG. & 6097 & 430 & 92 & 0 & .36 & 143 & 31 & 2 & 40 & 2350 & 0 & 251 & 81 & 2169 & 221 & 12751 \\
\hline
\end{tabular}

IWELVE MONIH SUMMARY

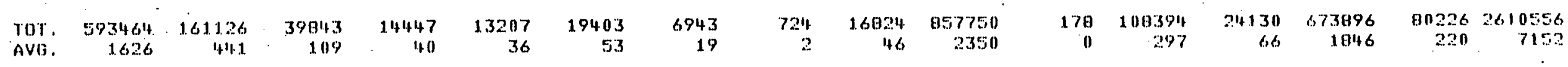




\title{
MUNICIPAL ELECTRICAL ENERGY COST \& CREDIT SUMMARY
}

\author{
ALTERNATES $1.01-8.13$
}

KWH KILOWATT-HOURS

KWHY KILOWATT-HOURS PER YEAR

KVACH KILOVOLT-AMP CORRECTIVE-HOUR(P.F.)

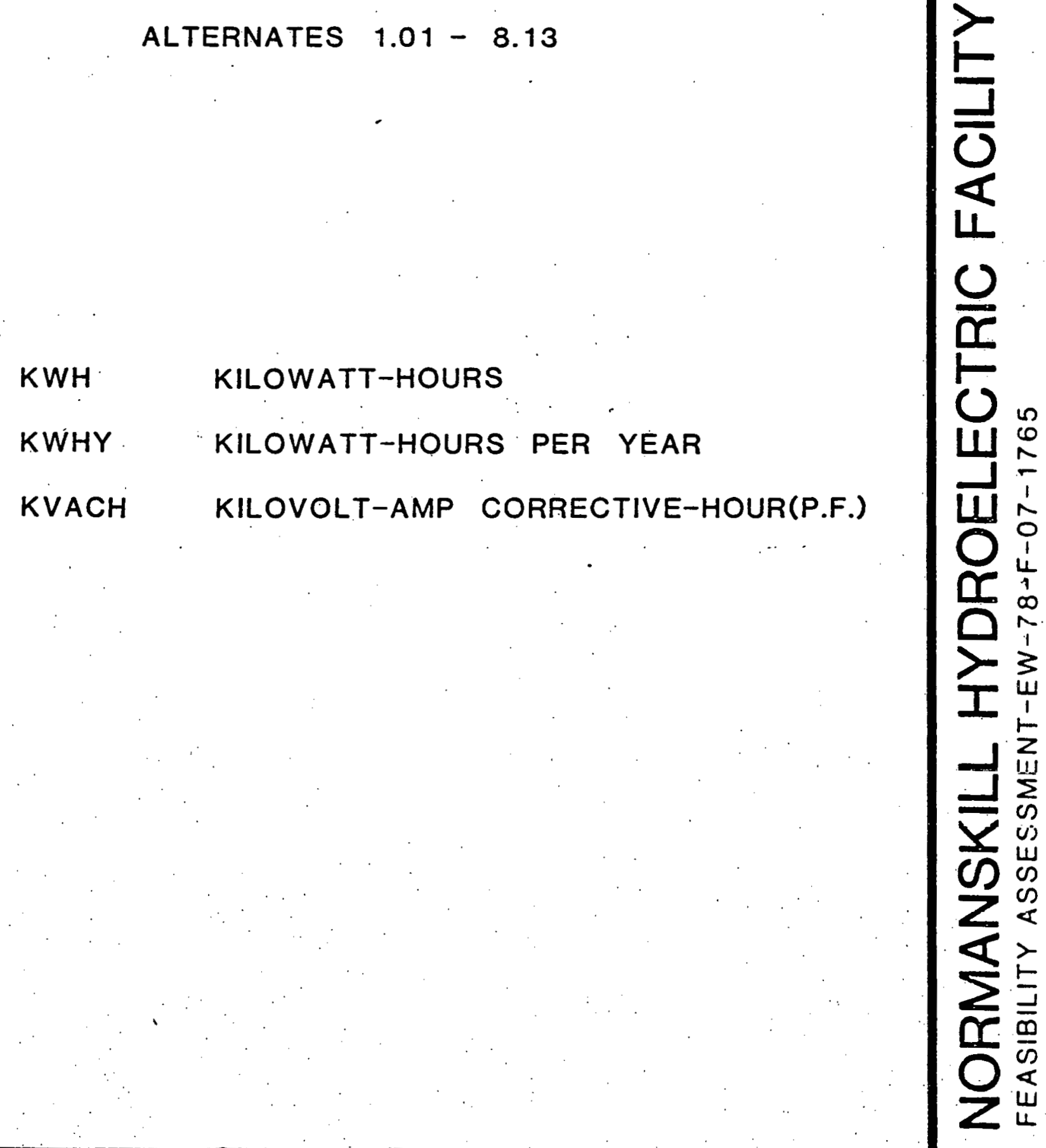


WATERVLIET HYDRO ELECTRLC FEASIBILITY STULY

ANNIJAL POWER COST \& CIREIIT SUMTAARY FOR VARIOUS ALTERNATIVE PROJECTS WAGEI ON ËO YEAR INFLOW STHUI.ATTON WTTH 6 MGIS WATER SUPPLY

AL.TERHATIVE H

\begin{tabular}{|c|c|}
\hline & 1.01 \\
\hline T. NO & \\
\hline T.NO & 1.03 \\
\hline$T . N(I)$. & \\
\hline T. TO & 2.112 \\
\hline T.NO. & 2.03 \\
\hline T.NOS & 2,04 \\
\hline$r . \mathrm{NO}$ & 2.105 \\
\hline T.NO. & 2.06 \\
\hline T.NOO. & 2.07 \\
\hline T.NO & 2.08 \\
\hline$T . N O$. & 2.109 \\
\hline T.NOI. & 2.10 \\
\hline T.HO & 2,11 \\
\hline T.NO & 2.12 \\
\hline T.NO. & 15.011 \\
\hline ' & $1+.02$ \\
\hline T.NO & 14.03 \\
\hline . & 4.04 \\
\hline T.NO. & 4.05 \\
\hline . NO. & 4.06 \\
\hline .110. & 4.07 \\
\hline ו. IO. & 4.08 \\
\hline T.NO. & 4.09 \\
\hline . NO & 4.10 \\
\hline . NO. & 4.11 \\
\hline .NO. & 5.111 \\
\hline $\mathrm{r} . \mathrm{NO}$. & 5.112 \\
\hline NO. & 5.03 \\
\hline NOS. & 5.114 \\
\hline NO. & 5.115 \\
\hline r. No. & 5.06 \\
\hline T.NO , & 5.07 \\
\hline & 5.01 \\
\hline , & 5.119 \\
\hline & 5.1 .0 \\
\hline & 5.11 \\
\hline & 5.12 \\
\hline
\end{tabular}

CITRERT COSTS

CITY POWER PWR COST

USE (KWHY) 5.35 (KWH

\begin{tabular}{|c|c|}
\hline 2611000 & 136367.24 \\
\hline $26110 \mathrm{A0}$ & 138387.24 \\
\hline 2611090 & 138387.24 \\
\hline 2611080 & 1.393897 .24 \\
\hline 2611000 & 1383897.24 \\
\hline 2611090 & 138387.24 \\
\hline 2611080 & 1383387.24 \\
\hline 2611060 & 136397.24 \\
\hline 2611080 & 138387.24 \\
\hline 26110011 & 1333367.24 \\
\hline 2611080 & 1.30387 .24 \\
\hline 261.1000 & 136307.24 \\
\hline 2611000 & 1.38367 .24 \\
\hline 261.1080 & 1319387,24 \\
\hline 2611090 & 1303137.24 \\
\hline 261.1080 & 136367.24 \\
\hline 2611080 & 1363137.24 \\
\hline 2611080. & 130307.24 \\
\hline 2611000 & 138.367 .24 \\
\hline 2611080 & 136387.24 \\
\hline 2611000 & 136367.24 \\
\hline 2611060 & 1383837.34 \\
\hline 2611060 & 1303137.24 \\
\hline 261.1080 & 1318367.24 \\
\hline 2611000 & 1.38367 .24 \\
\hline $26110 \% 0$ & 138387.24 \\
\hline 2611000 & 136387.24 \\
\hline 26111180 & 136397.24 \\
\hline 2611090 & 136387.24 \\
\hline 2611090 & 136307.24 \\
\hline 2611060 & 1363197.24 \\
\hline 2611000 & 136397.24 \\
\hline 2611080 & 136397.24 \\
\hline $261 \mathrm{~d} 0 \mathrm{\theta 0}$ & 136387.24 \\
\hline 2611000 & 1343307.24 \\
\hline 2611080 & 1363137.24 \\
\hline 2611080 & 1.3633137 .24 \\
\hline 2611080 & 1313387.24 \\
\hline
\end{tabular}

CREDITS FOR ALTERNATIVE POWER GENERATION GCHEMES-

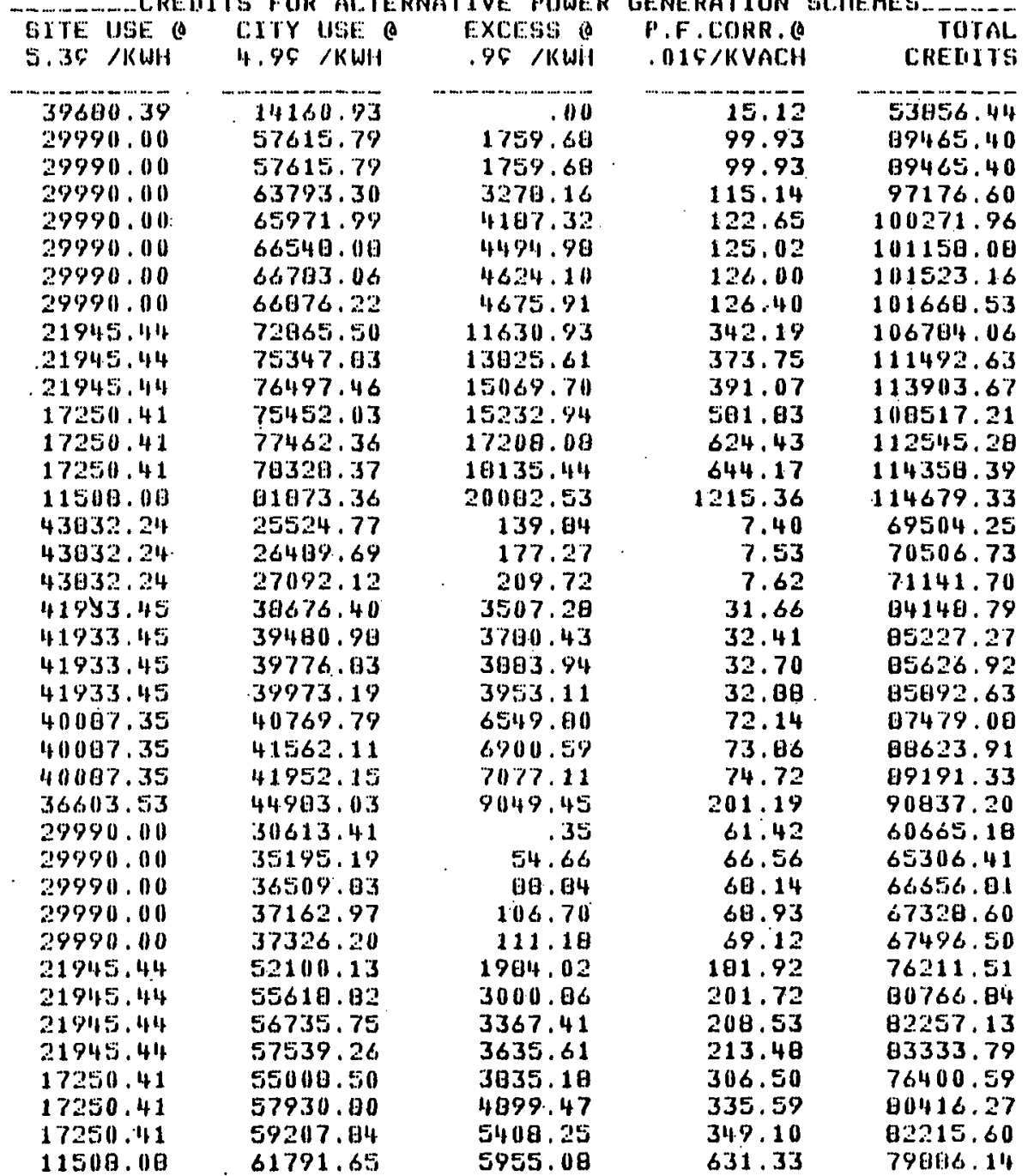

NE: I COS1 48921.84 48921.84 41210.64 38115.28 37279.16 36064.06 36718.71 31603.18 26894.61 24.483 .57 $29870: 03$ 25841.90 24028.85 23707.91 68882.99 67800.51 67245.54 54238.45 53159.97 53159.97 52760.32 . 521194.61 50901.16 19763.33 49195.91 47550.04 77722.06 73080.83 71730.43 71050.64 70890.74 62175.73 57620.40 56130.11 55053.45 61986.65 57970.97 56171.64 
WATERVLIET HYIIRO-ELECTRIC FEASIBILITY STURYY

ANNUAL POWER COST \& CREDIT EUMMARY FOR VARIOUS ALTERNATIVE PROJECTS

GASED ON 50 YEAR INFLOW GIMULATION WTTI O MBIO WATER SUPPLY

\section{ALTERIVAT IVE}

ALT.NO, B. O

ALT.NO. $\quad 0.02$

AL.T.NO. $6 \cdot 0.3$

ALT.NO. B. B.

ALT.NO. S.D

ALT.NO. B.O

AL.T.NO, 6.07

ALT.NO. E. EO

AL.T.NO, E.10

ALT,MO 6.11

ALT.NO. 6.12

ALT.NO. E.I

ALT,NO, 7. II

AL.T.NO, T.02

ALT.NO. T.0.3

AL.T.RO, 7.011

ALT 40.70

AL.T.NO. 7.07

ALT.NO. T.0B

AL.T.NO: 7.108

ALE.NO. 7.10

AL.T.NO. 7.11

AL.T.NO. 7.1

ALT.NO. 7.1

ALT. NO. $\quad 8.02$

ALT.NO. B:0:3

ALT.NO, B. 11

ALT, NO. $\quad 0.05$

ALT.NO, $\quad 0.06$

ALT.MO, B.OT

AL.T.NO. B. OB

ALT.NO, B.09

AL.T.NO, $\quad 0.10$

AL.T.NO. H.11

ALT.NO, G.1.2

ALI.NO. B.13

\begin{tabular}{|c|c|}
\hline $\begin{array}{l}\text { CITY POWEA } \\
\text { USE (KWHY) }\end{array}$ & $\begin{array}{l}\text { CosTs } \\
\text { PWR cost } \\
5.34 / K W H\end{array}$ \\
\hline 2611000 & 1363137.24 \\
\hline 2611060 & 1.38387 .24 \\
\hline 2611080 & 138387.24 \\
\hline 2611080 & 138367.24 \\
\hline 2611000 & 138.367 .24 \\
\hline 2611080 & $138387: 24$ \\
\hline $261.1080 \mathrm{k}$ & 138387.24 \\
\hline 2611000 & 138337.24 \\
\hline 2811080 & 138387.24 \\
\hline 2611000 & 138307.24 \\
\hline 2611080 & 138387.24 \\
\hline 2611080 & 138387.24 \\
\hline 2611000 & 13193937.24 \\
\hline 2611090 & 138387.24 \\
\hline 2611000 & 138367.24 \\
\hline 2611000 & 1363397.24 \\
\hline 2611000 & 138387.24 \\
\hline 2611080 & 1383397.24 \\
\hline 2611080 & 136387.24 \\
\hline 2611000 & 138367.24 \\
\hline 2611080 & 1313.3137 .24 \\
\hline $2 \measuredangle 11080$ & 138387.214 \\
\hline 2611060 & 136397.24 \\
\hline 2611000 & 1.38387 .24 \\
\hline 2611080 & 138367.24 \\
\hline 261.1000 & 139397.24 \\
\hline 2611080 & 1383367.214 \\
\hline 2611080 & 138387.24 \\
\hline 2611001 & 1336387.24 \\
\hline $2611090^{\circ}$ & 130387.24 \\
\hline 2611000 & 1333397.24 \\
\hline 2611080 & 138387.24 \\
\hline 2611000 & 1339387.24 \\
\hline 261.1090 & 138387.24 \\
\hline 2611000 & 134387.24 \\
\hline 2611000 & 138387.24 \\
\hline 2611060 & . 139397.24 \\
\hline 2611060 & 139387. \\
\hline & 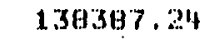 \\
\hline
\end{tabular}

_CREDITS FOR ALTERNATIVE POWER GENERATIOH SCHEMES.

CITY UGE O

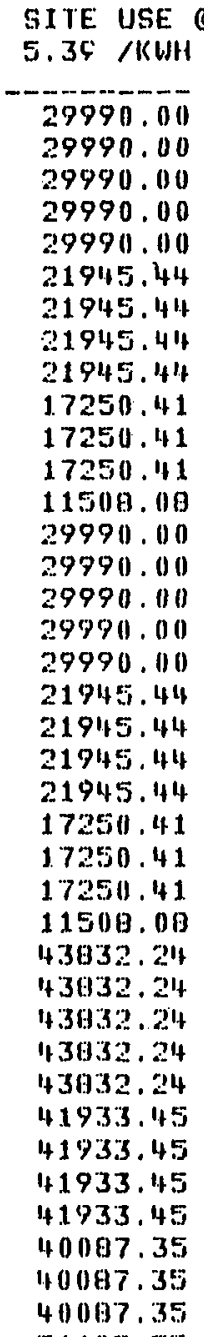

$4.96 / K 6 H$.

EXCESS
$.96 / \mathrm{KWI}$

P.F.CORR.
. OIS/KVACH

$59 \overline{87.63}$

65325.147

66540.00

67015.95

67201.014

69099.47

74225.83

75897.14

76536.73

72076.15

76261.03

77802.16

79376.81

52279.97

56236.62

57405.11

57626.29

501036.77

65725.52

60524.27

695141.49

69977.55

6132219.137

$70606 \cdot 968$

71634.30

74569.78

52393.22

54301 . 84

54074,32

55109.13

55202,45

55355.74

57390.89

50086.84

53355.80

57147.73

50010.95

509469.29

61445.42

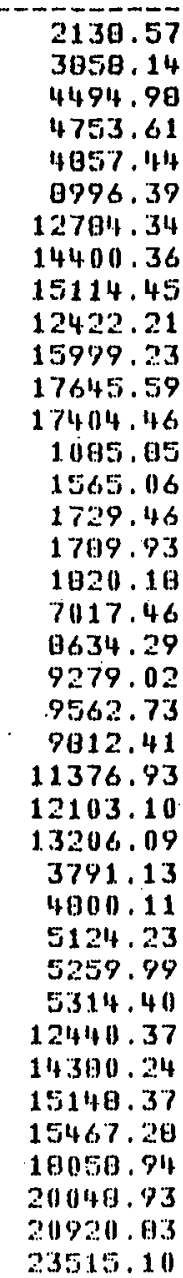

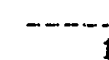

104.48
120.08
125.02

125.02

126.99

127.78

302.59

358.90

381.79

391.69

51.9 .49

590.146

633.78

1112.54

112.54

97.37

79.54

100.33

100.72

271.65

271.65

297.102
306.93

306.92
311.25

311.25

495.60

512.22

84.97

12.58
13.40

13.66

13.77

13.81

53.11?

57.22

80.82

59.40

122.39

131. 33

133.77

3511,76
CHEIITS

92100.68

99293.69

101158.08

10)10日6. 55

102176.26

100343.09

109314.56

112624.73

113908.31

102268.26

110109.13

113411.74

109401.96

83446.17

87809.05

09224.11

99706.55

89947.67

94960.07

$991+01.02$

101072.07

101.796 .97

95750.92

99729.82

101500.03

100229.92

100029.17

102917.39

103944.15

104215.13

104362.90

109782.65

1) 3769.80

115227.48

115316.111

115416.41

119077.56

120610.24

121514.01
NFI

cost

46266.56

39093.55

37229.16

36500.67

36210.90

380113.35

29072.68

25762.51

21398.93

36118.98

20278.11

24475.31

29905.20

54941.07

511498.1 ?

49163.13

48690.69

40439.57

43427.17

30906.22

37314.37

36570.27

$4: 2636.32$

$38657.4 \%$

36897.21

38157.32

36358.07

35139.85

34542.79

34172.11

34021.34

296114.57

21617.41

23159.76

22571.23

22970.83

19309.68

17177.00

$1617 ? .13$ 


\section{CONFIGURATION}

\section{Existing Facilities}

The Watervliet Reservoir impoundment is located on the Normans Kill at French's Mills, approximately 3.5 miles south of. Schenectady in Albany County. The dam is approximately 40 feet high impounding a usable storage volume of 1.6 billion gallons. The dam is owned and operated by the City of Watervliet, New York. The reservoir serves as a water supply reservoir for the city of Watervilet and the Town of Guilderland, New York. The dam is classified in the high hazard potential category under the National Dam Inspection Act, Public Law 92-367 due to a pump station and several homes. downstream that would be affected by a breach of the dam. The dam was designed by Solomon, Norcross \& Keis, Engineers of Atlanta, Georgia in 19.15 and built in approximately 1916. The dam is of a hollow Amburssen type structure comprised of slab and buttress construction. The length is 380 feet. The upstream face is sloped at 1.0 on 1.0 and the downstream face 1.7 (vertical) on 1.0 (horizontal). The total. length of spillway weir is approximately 324 feet. Crest elevation (concrete) is 256 local datum with the temporary flashboard elevation at 259 local datum. A 5 foot diameter conduit through the dam near its southern end is controlled by a 4 foot sluice gate. Invert of this conduit (mud valve) at its upstream end is elevation 222.6 local datum. A 50 inch steel penstock passes through the dam near its northern end and is controlled by a 48 inch single gate valve. The centerline of the penstock in the upstream face of the dam is elevation 240 local datum. Extensive memoranda are available detailing the initial design stages and discussions held with the New York state Water Resources Commission (previously New York State Conservation Commission) from the years 1900 to 1916.

The contract drawings of the dam are available as are additional drawings and specificatlons issued by the original dam designer, Solomon, Norcross, \& Keis, Consulting Engineers, V. G. Lamb, City Engineer of Watervliet, and this office. Modifications 
and rehabilitation of the dam have been limited to construction of $a$ training wall at the north abutment shortly after 1916 due to erosion in that area due to heavy spillway flows. This training wall was again raised 2-3 feet in 1955, again due to erosion of the adjacent streambank due to heavy spillway flows. A new concrete apron was added just downstream of the original structure in 1936 and in 1965 the spillway and concrete apron were resurfaced with gunite. The 30-36 inch high flashboards are replaced every 2-3 years, the last replacement in the spring of 1978.

The dam has been the focus of a Phase I Inspection Report as part of the National Dam Safety Program. This report was submitted to the U. S. District Corps of Engineers by Tippets-Abbett-McCarthyStratton on July 5, 1978. The Corps of Engineers report revealed several hazardous conditions at the dam which were immediately corrected by the City of waterviliet. The main deficiency that has been corrected is replacement of a 4 foot long section of penstock inside the dam adjacent to the intake valve. This section was heavily corroded and was replaced with new $3 / 8$ inch thick welded steel penstock on June 23,1978 . Also noted in the dam safety. report was the possible spalled condition of the concrete apron on the dam and spalling of the spillway surface. At the time of the National Dam Safety Report, access to the dam was hindered by high flows. Subsequent to the issuance of the report, the New York State Department of Environmental Conservation Dam Inspection Bureau has made a detailed inspection of the face and given the apron and spillway surface a clean bill of health.

The penstock connecting the dam with the existing pumping station was originally constructed in 1916 of $1 / 4$ inch thick riveted steel pipe. The initial section of penstock from station 00 to station $4+72$ is of original construction. Documents available indicate that this buried section was re-excavated from 1950 to 1952, scraped, cleaned and recoated with bituminastic material. The elevated section from station $4+72$ to $6+25$ was substantially rebuilt and replaced in 1960 following severe undermining and deterioration of concrete pier supports during a heavy flood. The 
steel surge pipe connected to the penstock at elevation $6+25$ was also. replaced in 1960 and is comprised of $1 / 4$ inch welded steel pipe, 4 foot diameter, carried up to elevation 270 local datum. The surge pipe is securely anchored in the rock ledge at this location. The section of penstock from elevation $6+25$ to approximately $9+$ 00 is again of original riveted steel construction. This section of penstock passes beneath the Town highway and is known to be in poor condition. The remainder of the penstock from station $9+00$ to termination at the manifold at station $14+28$ is of new construction, replaced in approximately 1955, is elevated and of $1 / 4$ welded steel construction.

Ultrasonic testing of the older sections of the penstock (those buried sections of riveted steel construction) was performed on June 23,1978 . It was found that the original $1 / 4$ steel plate was corroded in areas, leaving a resulting thickness of 0.100 inches minimum. Due to this severe deteriorated condition, any considerations for reuse and adaption of the existing faciilities has included replacement of the original riveted steel penstock sections with new $1 / 4$ inch thickness steel penstock of welded construction.

Running parallel to the 48 inch penstock is a 10 inch cast iron water main, supplying spring water from a spring at the reservoir periphery to the pumping station. It has been determined that this 10 inch cast iron main is severely tuberculated, is of inadequate hydraulic capacity to supply water for pumping purposes alone and thus is no consequence in any adaptation or rehabilitation effort.

The existing water pumping station was constructed in 1915 under the original water supply contract. The original construction entailed acquisiton of two horizontal francis turbines directly connected to two-stage centrifugal pumps to supply water to the City via a transmission main extending across the Towns of Guilderland and Colonie, New York to the City of Watervliet. Little is known of the original turbines. No provisions were made in the original construction for electrical pumping, however from records of the 
N.Y.S Water Resources Commission, it is assumed that electric pumping capabilities were added several years later following the drought of 1917. At that time the Watervliet reservoir was completely emptied by February of 1918 due to unregulated use by the City of water for pumping purposes. The existing machinery located at the pumping station is comprised of two $\mathrm{S}$. Morgan Smith horizontal Francis turbines connected through gear boxes to single stage Delaval centrifugal pumps. The original turbines were apparently replaced as part of a WPA project in 1933. It is uncertain what necessitated the replacement of the original units: From correspondence dating to that time, however, it is known that some difficulties were encountered in the installation and initial operation of the replacement units due to problems in cooling and lubricating the gear increasers. The turbines are designed to operate under 62 feet of head at 900 RPM. The single runners are each 12-3/4 inches in diameter and are horizontal type, with steel cases. Hydraulic curves and original test data for the turbines is available. Auxiliary pumping is provided at the station by three identical Delaval centrifugal pumps powered by $100 \mathrm{kva}$ Westinghouse induction motors. The existing hydraulic turbines, although continuing to operate satisfactory 24. hours a day are approximately. 45 years old and nearing the end of their useful economic life. Recommendations following for utilizing the existing facilities require rebuilding of the units to original specifications to provide for adequate reliability.

Additional features of the additional pumping station include transformer bank supplying standby power to electric pumps. This transformer bank and associated electric switch gear is approximately 50 years old and is of open front construction. Due to safety, space, and reliability considerations, all the alternatives developer that include the existing facilities must also include replacement of this electric switch gear. Internal piping at the pumping station is of cast iron construction. It is in generally fair condition although all flange connections should be rehabilitated as a prerequisite for any rehabilitation of the facility. 
The pumping station structure is of reinforced concrete foundation with a. 12 inch triple course brick wall superstructure. The roof is framed with steel angle trusses approxlmately $9^{\prime} 7 "$ on center, supporting channel purlins, with 2 inches of subroofing and slate roofing. The building is of substantial construction although rehabilitation of windows, doors, roofing, and heating and ventilating systems will be required to extend the life of the structure for 20 plus years.

All elevations pertinent to this project have been field verified and the correspondence between the local. (Keis) datum and. the USGS datum has been determined. A listing of all pertinent: elevations and tail water and head water assumptions follows.

\author{
DESIGN ELEVATIONS \\ U.S.G.S Datum \\ (Keis datum equals USGS minus 3.0 feet)
}

\section{Point}

Top of flashboards ( $36 "$ ")

Top concrete dam

Invert mud valve conduit

Streambed at base of dam

Design tail water at base of dam

Streambed at first falls

Streambed below first falls

Design tail water below first falls

Streambed at second falls

Streambed helow second falls

Design tail water below second falls

Floor at existing pump station

Bottom streambed at existing tail race

Design tail at existing pumping station

Centerline existing horizontal turbines
Elevation

262.4

259.4

325.3

225

227

224.5

209.0

211.0

204.0

196.0

198.0

202.3

$192 .+$

$197 .+$

$202 . \overline{8} 3$

\title{
Site Options
}

As a prelude to determination of the optimum hydroelectric alternative for this site, a wide range of alternatives was developed. The only limitation at the initial stage for these alternatives was a maximum head limitation of 20 meters, ( 66 feet). Little penalty was extracted with this limitation since the stream 
gradient below the existing pump station is less than 0.0013 . Due to the congested area just downstream from the dam, several unusual configurations were developed. These congestions are due to two steel trestles of late Nineteenth Century vintage carrying mainline ConRail tracks across the Normans Kill, a county highway bridge and a relatively narrow streambed and gorge. A third additional consideration design parameter was the relatively high $(38,600 \mathrm{cfs})$ standard Project Flood [Ref. 102] for the site resulting from the 120 square mile watershed. The eight alternates initially chosen represent a broad range of possibilities not only for civil works but also for various machine configurations. A description of each option follows. Each option has also been schematically shown on an aerial photograph of the site (Figures $41 \mathrm{~A}-41 \mathrm{H}$ ).

Option One - This alternative would utilize the existing 48 inch steel penstock. Deteriorated sections of the penstock would be replaced with new 48 inch penstock of welded steel construction. The existing surge pipe would be retained. The existing intake would be improved with a new trash rack, a motorized valve operator and a new intake transition penstock. Location of the machines under Alternate one would be at the existing pump house. The three different machine types selected for analysis under this option are the existing horizontal Francis machine, a new larger horizontal Francis machine and a vertical axial flow propeller machine. Rationale behind selection of machines for this and other alternates are discussed further in the next section.

Option Two - Comprised of a siphon intake adjacent to the dam, this siphon intake would be a wholly separate structure from the dam and would be comprised of driven steel sheeting and reinforced concrete. The siphon intake would connect with a steel penstock located at the north end of the dam. The siphon penstock would extend along the side of the gorge, crossing the county highway, and terminating at a powerhouse at the north end of the second falls on the Normans Kill some 900 feet distant from the siphon intake. Due to the high side slopes at the site of the powerhouse, a vertical machine was judged to be most appropriate in this case. A Francis machine was selected for analysis. 
Option Three - Utilizing the existing mud valve and wasteway at the dam, this alternative was considered possible with the use of a bulb type or a horizontal axial flow tubular type turbine, utilizing the existing conduit and inlet valve. This option was eliminated after it was determined that the design of the existing conduit and mud valve were not adequate for pressurized and/or water hammer conditions that would occur under a hydroelectric generating scheme.

Option Four - Option Four would consist of a new gravity intake located in bay \#7 of the dam. The intake would be of reinforced concrete construction, approximately bay width ( 18 feet). The intake would consist of a stop log rack, motor operated sluice gate (head gate), manually cleaned bar rack and intake transition section. The powerhouse under Option Four would be within the dam with discharge from the turbine directly at the downstream face of the dam. The machine selected for inclusion under this alternate is a horizontal, axial flow, variable pitch propeller machine. Due to limited spillway capacity, it was felt that further reduction in the spillway capacity caused by construction of a conventional downstream face powerhouse suitable for vertical machines would not be appropriate.

Option Five - A siphon intake similar to that discussed under Option Two with the siphon penstock terminating at a powerhouse at the foot of the dam is included under Option Five This alternative was examined since it eliminated the necessity of crossing the ConRail right-of-way and the county highway although the approximate 35. feet of gross head was significantly less than site potential. option Six - A siphon intake similar to that discussed In Alternative Two would be constructed with a siphon penstock extending 1,750 feet to the site of the existing powerhouse. This extremely long penstock would be buried over the initial section and then run elevated for the final 1,000 plus feet. The powerhouse would consist of a new structure located adjacent to the existing pump station, housing either a horizontal or vertical francis machine or a horizontal axial flow propeller machine. 
Option Seven - This option includes a siphon intake connected to a siphon penstock terminating at the base of the first Normans Kill falls, at a powerhouse similar to that discussed under Option Two. This alternate was investigated due to the shorter length of penstock required as compared to Options Two and Six.

Option Eight - Option Eight is comprised of a new gravity intake as discussed under option Four at bay 5 of the dam. The intake would terminate in a new steel penstock exiting at: the base of the dam and extending for 900 feet down the center of the Normans Kill. This penstock would be constructed beneath the streambed by blasting the predominately rock streambed, installing the penstock, backfilling with concrete and replacing the rock streambed to its original condition. The penstock would terminate in an underground powerhouse located just upstream from the second falls. The powerhouse would house a horizontal axial flow, variable pitch propeller turbine with a discharge emanating at the base of the second falls. This alternate. was deemed particularly feasible and attractive due to the short (900 foot length) penstock and a relatively high (65 feet) gross head. It is also felt that the buried nature of the penstock and powerhouse would have positive environmental and aesthetic considerations. since the hydrologic phase determined that a wide range of flow considerations were available at the site, each alternate was thus then investigated for several different flows ranglng from 55 to 500 cfs in order to optimize penstock sizing in relation to hydraulic losses and cost. Each flow subalternate was again divided into various penstock size configurations. In all, 78 different combinations of site alternate, penstock size, design flow, and machine type were investigated.

\section{Machine Options}

Numerous turbine/generator manufacturers were contacted to ascertain availability of suitable equipment. The following suppliers were contacted with responses as indicated. Parentheses indicate number of different configurations upon which suppliers furnished cost data: 


\section{Supplier}

Escher Wyss Ltd. James Leffel Company

Mitsubishi Industries Ltd. Nohab Hydropower Division Alsthom Atlantic Inc. Sulzer Brothers Ltd. General Electric Company Allis-Chalmers Corp. Ossberger (Stapenhorst Inc.) Oy Tampella AB
Response as of $04 / 23 / 79$

not interested quoted (see General Electric)

no reply

no reply

no reply

see Escher Wyss

quoted ( 2)

quoted (10)

quoted ( 2)

quoted. ( 1)

The following machine types were examined for inclusion into the optimum configuration analysis:

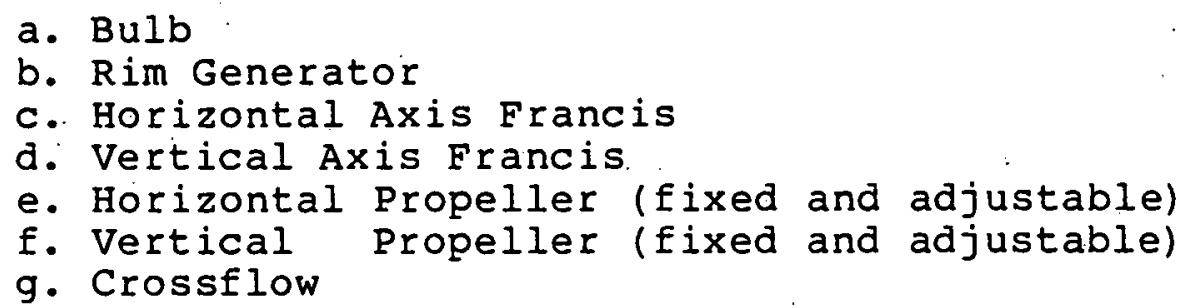

Preliminary analysis yielded the following:

The bulb type machine initially thought to be feasible for several of the siphon alternates was rejected for several reasons. Following inquiries to several manufacturers, it was determined that the size of the project and of the penstock passageways were inadequate for proper bulb design. In addition, the flow variability inherent in this "run of river" design and the relatively high (65 degree F) summer water temperature posed cooling problems.

The rim type generator was rejected due to the small size of the project and and thus the hydraulic passageways and by the lack of interest of any manufacturer in supplying such a machine. In addition, the highly variable water quality with springtime turbidities (particulate) exceeding 500 ntu would cause severe problems with the sealing surfaces at the periphery of the machine. problems with the bulb type machine. 
The cross-flow type hydraulic turbine was investigated and initially looked to be promising, however reliability considerations were the predominant reasons for rejecting the cross-flow apparatus from further consideration.

Multiple runner Francis machines were determined to be too complex for this size project. The remaining machines, horizontal Francis type, vertical Francis type, horizontal axial tube type, and vertical Kaplan type machines were considered. However, difficulties were encountered in obtaining any quotations for vertical Kaplan machines. A tabulation of the machine selection follows:

\section{Machine Type Selection}

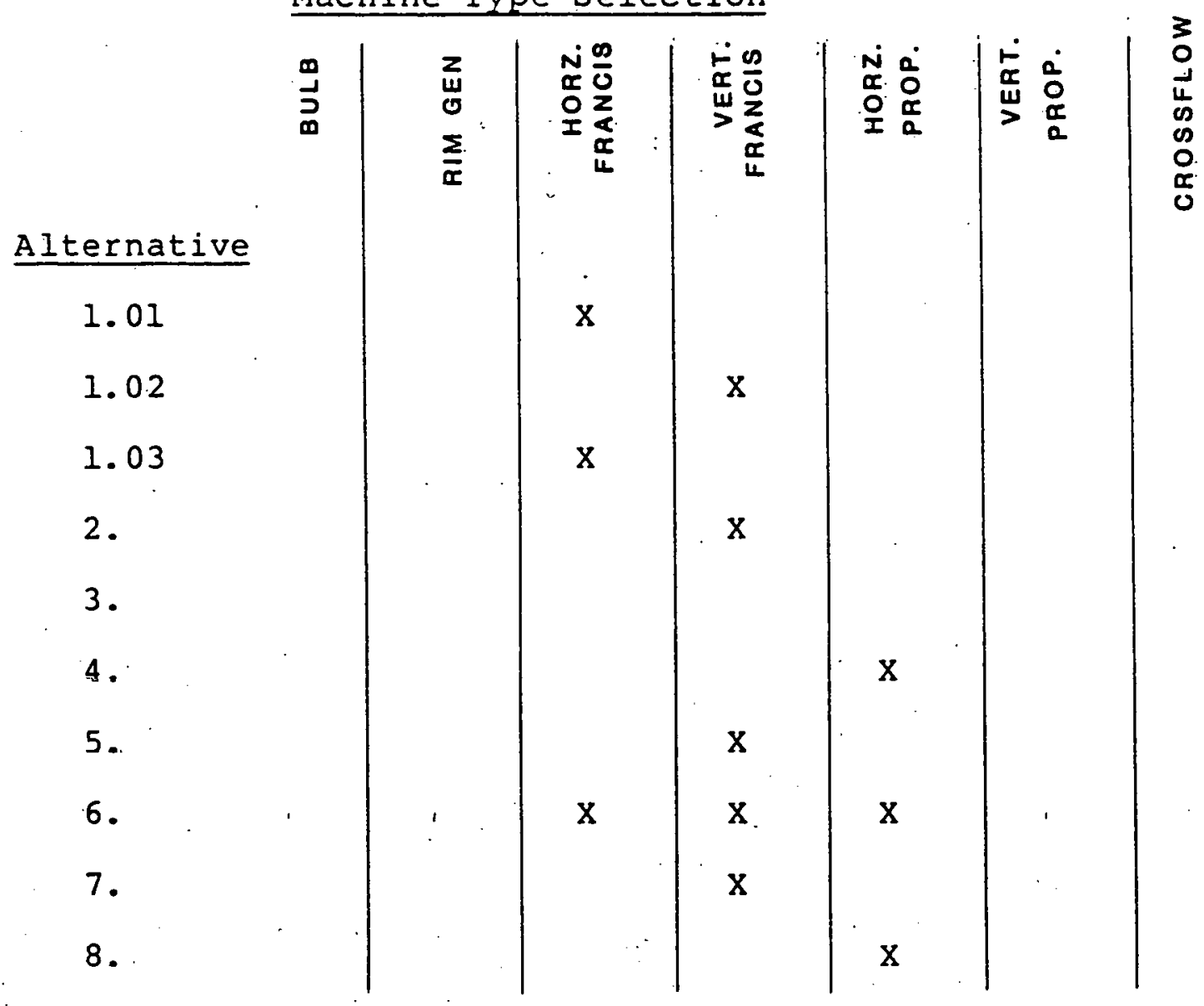

Comments: (1) Bulb turbines eliminated due to size limitations and potential cooling difficulties due to flow variability.

(2) Rim generator types eliminated due to size and abnormally high turbidity. 
(3) Cross-flow type eliminated due to reliability considerations.

(4) Multiple runner Francis type eliminated due to complexity.

\section{Costs}

Capital costs for the civil structures, machinery, mechanical works and equipment are estimated 1979 costs derived from manufacturers, suppliers, contractors, cost estimating manuals such as Dodge Construction Cost manual, and the firms bid award records. Costs included are for materials, labor, overhead and profit. For each of the 78 alternatives, costs were determined.

A specific typical cost breakdown for a given Alternate (8.07) is included at the end of this section. A description of the cost' estimating methods used follows:

1. Penstock - In Alternative No. 1, part of the existing penstock will be reused so the cost presented reflects replacement of only part of the penstock with new. fabricated steel pipe and rehabilitation of the remaining existing penstock. In all other options, the existing penstock would be abandoned and a new penstock constructed. Initially three penstock materials were considered; fabricated steel, reinforced concrete and concrete-lined rock tunnel. Unit cost for each is illustrated on Figure 42 .' Since the cost of lined rock channel and concrete pipe is substantially greater than steel, the penstock material considered in each of the alternatives is fabricated steel. The penstock cost. in each alternative is the proposed penstock length times the unit cost of steel pipe constructed under the appropriate specific conditions, that is underground in soil, underground in rock, etc.

2. Intake - The existing penstock intake, though adequate for its present use, is lacking in several important features. First, there are no practical means of routinely inspecting and cleaning the intake and bar rack. Second, there is no way of dewatering the section of penstock between intake and the gate valve for penstock or 
valve repairs. In addition, increasing the penstock size would dictate completely. rebuilding the existing intake at a cost not much lower than a new intake.

Initially it was thought that penetrating the dam for a new intake would entail construction difficulty. Therein was born the idea of a siphon intake located behind the dam with a penstock laid in the rock around the north abutment, as shown on Figures Nos. 410 thru $41 G$ for Alternatives Nos. 2, 5, 6 and 7, respectively. A siphon is necessary since the ground at the abutment is rock and is substantially higher than the dam crest. Gravity flow around the dam would require a deep excavation in the rock abutment which could lead to potential seepage problems.

upon closer examination, it was determined that construction of a gravity intake integral with the dam would be feasible and would probably be more economical than constructing a siphon intake. A reassessment of the dam's structural stability and conversations with contractors experienced in this type of work now lead us to believe that construction of a dam integral intake would be feasible. The cost of both intake structures have been estimated using Lang factor methodology as follows:

The cost of the dam integral intake (Options 4 and 8 ) is for the most part fixed. The intake will be constructed between two existing dam buttresses so its external physical size, quantities of concrete and form work are constant. However, the sluice gate and bar rack sizes will vary with flow. Intake costs for various flows (Q) were derived from the following equation:

(I) Intake cost (Dam Integral) $=\mathrm{K}\left(1243 \mathrm{Q}^{0.475}\right.$

$$
+60.3 Q+\$ 205,545) \text { : }
$$

$\mathrm{K}$ is a constant between 1.15 and 1.25 relating overhead and profits to capital costs. The first term of the expression within the brackets relates cost of the sluice gate to flow. The second term relates the cost of the bar rack to flow. The third term is a constant which includes the costs of all labor and other materials.

For the siphon intake, the same assumptions regarding the bar rack and sluice gate were made. In addition, the width of the 
structure will vary with the siphon diameter, so concrete costs can also be related to $Q$. The following equation gives costs for the siphon intake:

(2) Intake cost (Siphon) $=\mathrm{K}\left(12430^{0.475}+60.3 Q+\right.$
$\left.1720\left(Q^{0.5}\right)+\$ 292,480\right)$

The third term of the expression within the parenthesis relates cost of concrete to flow, that is, Q. The constant term in this expression is substantially larger than in equation No. I due to the sheeting cost involved in this intake.

Rehabilitation of the existing intake to accommodate Option No. 1 is estimated to cost about $\$ 33,000$. This includes a new bar rack, a motorized valve operator along with the replacement of the short piece of penstock between the valve and the intake.

3. Powerhouse - Powerhouse size for a Francis-type turbine (Options $1,2,5,6,7$ ) is related directly to the physical size of the machine, that is, the turbine. An indicator of machine size is runner diameter. Powerhouse costs for several machine sizes were determined through ordinary estimating methods and these costs were related to runner. diameter using Lang factors.

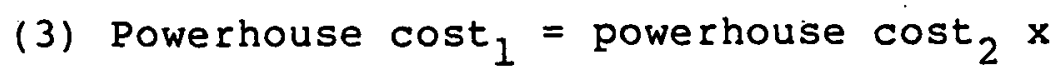

$$
\left(\text { Diameter } 1 / \text { Diameter }_{2}\right)^{1.35}
$$

The powerhouse for Alternate No. 4 will be inside the dam. Since it is a tube-type installation, the cost is not expected to vary with machine size. It is estimated to be a constant $\$ 325,000$. This cost includes the construction of a superstructure exterior to the dam to allow for access to the powerhouse.

The powerhouse for option No. 8 will be constructed in rock beneath the streambed with an access tunnel also beneath the streambed leading to the north bank of the stream. The entire structure would, in essence, be a concrete-lined rock tunnel. Form work and exterior finishing will be minimal. The cost for this structure is estimated at $\$ 170,500$.

The cost of modifying the existing pump station to serve as a powerhouse for option 1.01 would include the cost of removing the two existing hydraulic pumps, modifying the existing piping, 
installing an adequate heating, ventillating and air-conditioning systems and constructing stands for the two new generators. Approximate cost for this work will be $\$ 45,000$.

In option 1.02, the existing hydraulic pumps and turbines would be removed and replaced with a new vertical Francis turbine and generator. The cost of modifying the structure for this purpose is $\$ 96,000$.

Option 1.03 replaces the existing turbines and pumps with a horizontal Francis turbine and generator. This will require a new draft tube and modification of the tail race at an estimated cost of $\$ 96,000$.

4. Switch Gear - Switch gear includes all those electrical devices necessary to transport power from the point of generation to the point of use. Included are such items as circuit breakers, relays, transformers, switchboards, etc. These costs were separated into two categories, constant costs and variable costs. Constant costs include costs for certain relays, switches and transformers, metering, utility tie, and a station battery.

Variable switch gear costs such as the cost for certain transformers and switchboards were related to power production by Lang factors by the following equation:

(4) Cost $_{1}=\operatorname{cost}_{2}$ (power ${ }_{1} /$ power $_{2}$ ) $x$ where $X$ varies between 0.24 and 0.8 depending upon the type of equipment being estimated.

The cost of the transmission line from the powerhouse to the existing pump station and utility tie is given by the following equation:

(5) Cost $=50$ (1500 - penstock length)

The total switch gear cost is the sum of all variable costs as given by the above-mentioned equations plus a constant cost. The general equation for arriving at total switch gear cost is:

(6) Switchgear cost $_{1}=$

Transformer Cost 2 (Power /Power $_{2}$ ) $0.7997+$ Switchbuard Costs ${ }_{2}$ ( Power $_{1} /$ Power $_{2}$ ) $0.2427+$ 50 ( 1500 - penstock length) + Constant 
5. Machines - Machine costs in this report refers to costs for both generators and turbines, including any miscellaneous equipment such as speed increasers, exciters, etc. The cost for variable pitch axial flow turbines (Options 4 and 8 ) were derived from cost estimates of various size machines provided by the Allis-Chalmers hydroturbine division. However, these quotes included certain switch gear costs which had to be disaggregated in order to determine bare machine cost. From the eight costs quoted on eight different size machines, the following exponential equation relating power to machine cost (for machines of this type).

(7) ${\text { Machine } \text { cost }_{1}=\text { Machine cost }}_{2}\left(\right.$ powe $_{1} /$ Power $_{2}$ ) 0.495

This equation includes certain switch gear costs, both constant and variable, which must be subtracted in order to arrive at a bare machine cost.

(8) Bare machine cost $1=$ Machine cost $_{2}$ (Power ${ }_{1} /$ Power $_{2}$ ). 0.495 Transformer costs 2 (Power 1 /Power (P) $^{-}$ Switchboard costs 2 (Power ${ }_{1} /$ Power $_{2}$ ) 0.2427 50 ( 1500 - penstock length) - Constant

Machine costs for Francis-type turbines (Options 1, 2, 5, 6 and 7) were derived from costs provided by the General Electric company. In this case as in the case of the axial flow turbines, an equation was derived to relate cost of machine to the power produced.

(9) Machine cost $_{1}=$ Machine cost ${ }_{2}$ (Power $_{1} /$ Power $_{2}$ ) 0.495

However, the quote did not include a cost for a inlet valve ahead of the turbine. Costs for several sizes of hydraulically operated butterfly valves were obtained and an equation exponentially relating penstock diameter to valve cost was derived. The total Francis machine cost (inlet valve cost) is given by Equation No. 9 A.

(9A) Francis machine cost $1=$ Francis machine cost 2 (Power ${ }_{1} /$ Power $_{2}$ ) + valve cost 2 (penstock dia. 1 /penstock dia. $\left.{ }_{2}\right)^{1.25}$ 
6. Ancillary Equipment - Certain ancillary equipment, not directly related to the production of power, is necessary for the operation of the hydroelectric facility. This equipment includes safety and firefighting apparatus, hoists, telemetry, tools, small power generators, etc. The cost for such equipment is estimated to be a constant $\$ 74,000$ for all alternatives.

7. Mobilization and Temporary Services - The cost of contractor mobilization is estimated to be approximately $5 \%$ of the estimated capital cost of the preceding Items, 1 thru 6. Also included in this item is the cost for Temporary Services such as a water supply line and temporary pumping power when required.

8. Administration, Legal and Technical - Also included in the total project capital cost is a cost for design administration, legal services and engineering investigation. These costs were divided into three categories: 1. Design. 2. Legal and administrative. 3. Engineering investigation. Design costs are based on a percentage of capital costs as recommended by ASCE Manual No. 45. The cost of engineering investigations such as field. surveys, licensing report, geotechnical surveys, etc. are neither site specific nor dependent upon the quantity of power produced and will be included as a constant lump sum for all alternatives. Legal and administrative fees were estimated to be approximately 18 of the sum of the capital, design and investigation costs. The total cost for this item can be expressed as a function of capital costs plus a constant by the following equation:

(10) Administration, legal and technical cost $=$

0.747 (capital cost) $0.838+$

.01 (capital cost) + Constant

The following cost tabulations for a typical alternative (in this case 8.07) are furnished as examples. 
TYPICAL INTAKE COST BREAKDOWN

(Alternative 8.07 )

Item

Sheeting, bracing \& dewatering

Concrete

Bar rack

Miscellaneous metals

Electrical facilities

Miscellaneous

Sluice gate, $8^{\prime} \times 8^{\prime}$, incl. motor operator

SubTotal

Plus $10 \%$ contingencies

Total Cost

\section{Quantity}

70 tons

300 C.Y.

$\mathrm{L} . \mathrm{S}$.

8 tons

L.S.

I.S.

I.S.

Unit Price

$\$ 1,520$

$\$ 250$

$\$ 13,400$

$\$ 3,000$

$\$ 8,000$

$\$ 15,000$

$\$ 21,000$

$\$ 21,000$

$\$ 264,800$

26,480

$\$ 291,280$ 


\section{Typical Switch Gear cost Breakdown \\ (Alternative 8.07)}

\section{Item}

$15 \mathrm{KV}$ horn gap switch

125 AMP $15 \mathrm{KV}$ circuit breaker

2,000 KVA transformer

600 AMP $5 \mathrm{KV}$ air circuit breaker

10 AMP $5 \mathrm{KV}$ air circuit breaker

30 KVA dry transformer

40 AMP $5 \mathrm{KV}$ air circuit breaker

100 AMP $5 \mathrm{KV}$ air circuit breaker

Current transformers

Power transformers

Protective relays:

type 25

Type 27

type 50

type 51

type 51G

type 5 iM

type 81

type 87
Quantity

3

1

1

2

2

1

2

2

21

12

1
1
2
3
2
1
1
1

Metering

L.S.

Utility tie

L.S.

station battery

Overhead line

2400 volt switchboard

900

1

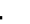

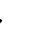

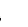

12,000

12,000

2,400

12,000

12,000

375

450

750

570

500

500

500

500

525

875

6,000

7,500

12,000

50

35,000
Cost

$\$ 4,500$

17,000

30,000

24,000

24,000

2,400

$24,0.00$

24,000

7,875

5,400

750

470

1,000

1,500

1,000

500

525

875

6,000

7,500

12,000
45,000

35,000 
Miscellaneous

Subtotal

314,295

Plus $10 \%$ contengencies

31,429

Total Amount

$\$ 345,724$

Typical Powerhouse Cost Breakdown

(Alternative 8.07 )

\section{Item}

\section{Excavation}

Constructural concrete

Miscellaneous metals

Interior electric and

auxiliary generator

Piping

HVAC

\section{Quantity}

483 Cubic Yds. $\$ 50$

247 Cubic Yds. 200

7 tons

L.S.

I.S.

L.S.

3,000

36,000

5,000

18,000

Subtotal

Plus $10 \%$ contengencies

Total Amount

\section{Cost}

$\$ 24,150$

$\$ 49,400$

$\$ 21,000$

$\$ 36,000$

$\$ 5,000$

$\$ 19,500$

$\$ 155,050$

$\$ 15,505$

$\$ 170,555$ 
Optimization Methodology

The evaluation of the 78 alternative configurations initially suggested itself as a linear programming problem. However, after completing the marketing phase, the uniqueness of the value function became apparent, i.e., it was observed that the value of power generated depends upon whether the power is sold to the local utility, wheeled to the City, wheeled to other customers, or whether the machine was used simply for synchronous power factor correction. This uniqueness of the power value function causes abrupt discontinuities in the total project cost function, making the application of linear programming solutions difficult. Consideration was given to continuing along the linear programming methodology. line using the constrained linear system threshold concept developed by Todini and wallis. However, this approach was alsó abandoned. Instead, it was decided to develop an optimization matrix for a comparative value analysis.

Eight powerhouse site options were selected. Each of these options include six independent variables (design flow, penstock diameter, net head, intake type, machine type and machine efficiency) which results in a total of 78 alternate options. The 78 alternatives form the horizontal elements of the optimization matrix while 36 variables comprise the vertical elements (Figure 44). The vertical elements are as follows:

Line 1. Alternative number - The first number designates one of the eight powerhouse site options. The remaining decimal indicates the alternative number within that option.

Line 2. Design Flow (Q) - The specific flows used were 100, 200,300 and 500 cubic feet per second except for Alternative No. 1.01 which was defined as utilizing the existing machines with a flow of 35 cubic feet per second. 
Line 3. Gross Head ( $\mathrm{H})$ - defined as the relative difference in elevations between the top of flashboards and design tail water as previously listed under "Design Elevations."

Line 4. Penstock Diameter - The sizes considered were 48, 60, 72,84 and 96 inch penstock. Velocity limitation was 12 feet per second.

Line 5. Intake Area - Intake area was based upon a maximum velocity of 2.5 feet per second at the bar rack and 6 feet per second at the sluice gate. The magnitude of the intake area was a determinant in the intake cost, although not a large one.

Line 6. Net Head - Net head is the gross head minus all friction and minor losses such as at the entrance, valves and bends. The friction coefficient used in all cases was $C=100$. Lines 8, 9, and 10. Runner Diameter, Setting, Specific Speed These three parameters were used as determinants for the size of the structure and type of machine. These variables were calculated using the standard turbine homologous equations [Ref. 4]

Line 11. Unit Efficiency - total machine efficiency including turbine, gear box and generator for the various types based upon manufacturers' data. Unit efficiencies are comprised of machine efficiency at full load, gear box efficiency of .98 (if gear box is used) and a generator efficiency of .95. Unit efficiency as defined here includes all losses, including entrance and exit losses, at the turbine.

Line 12. Composite Efficiency - It is apparent from manufacturers' published data that machines, i.e., turbines and generators, operate at various efficiencies depending upon the given flow and the load. The composite efficiency is a weighed average efficiency based upon simulated flow patterns.

Line 13. Net Power - Net power is the net generating capacity at the bus bar for a given flow, net head and composite efficiency. This net power was also used in the benefit value program to determine the total benefits to be accrued from selling power. Line 14. Penstock Length - Scaled from $1 "=200^{\prime}$. New York State Department of Transportation photogrammetric maps.. 
Line 15 thru 25. These elements are the items of capital cost and have been explained previously in the costs section.

Iine 26. First Annual $O \&$ M Cost - This is the total annual cost in the first year for operating and maintaining the facility excluding labor cost.

Line No. 27. $O \& M$ Compound Cost Gradient - This variable is used to escalate the annual $O \& M$ cost by a fixed annual rate over the period of the analysis. Further explanation of this rate will be given in the Economic Analysis chapter following.

Iine No. 28. Present Worth of 50-Year O\& M Cost - This is the the present value of the total amount to be disbursed for $O \& M$ over the 50-year period discounted with the stated discount rate.

Line No. 29. Present worth of Costs - The sum of Lines 25 and 28.

Line No. 30. Annual Value of Energy Generated - This number represents the value of the power used by the City in place of power currently purchased from Niagara Mohawk Power Corporation plus additional revenue from the sale of excess power to Niagara Mohawk. This number has been previously calculated in the benefit value program (see Marketing chapter).

Line 31. Energy Compound Value Gradient - This compound amount factor reflects the projected annual increase in electrical energy cost.

Line 32. Present Worth of 50 Years of Energy - The total value of all energy produced by the facility in the 50-year service life, reduced to a present value using the assumed discount rate. Line 33. Present Worth of Residual Value - Since the analysis period and the assumed equipment life are equal ( 50 years) by definition, the residual or the remaining value of the project at the end of 50 years is zero in the example depicted as figure 44 .

Iine 34. Present Worth of Credits - The sum of Lines 32 and' 33.

Iine 35. Present worth of Net Cost - This is the total project cost minus the total credits derived from power production (Line 29 minus Line 34 ). 
Line 36. Ranking by Lease Net Cost Present Worth - The alternatives are ranked No. 1 through No. 78 , No. 1 being the alternative with the least net cost and therefore the optimum alternative.

This program was run for two classes of projects, one being the rehabilitation of existing facilities and the other being construction of a completely new facility. The rehabilitation class includes option No. 1. The new construction class consists of Options No. 2 through No. 8. An optimum alternative from each of these classes has been selected and will be discussed further in the Economic Analysis. 


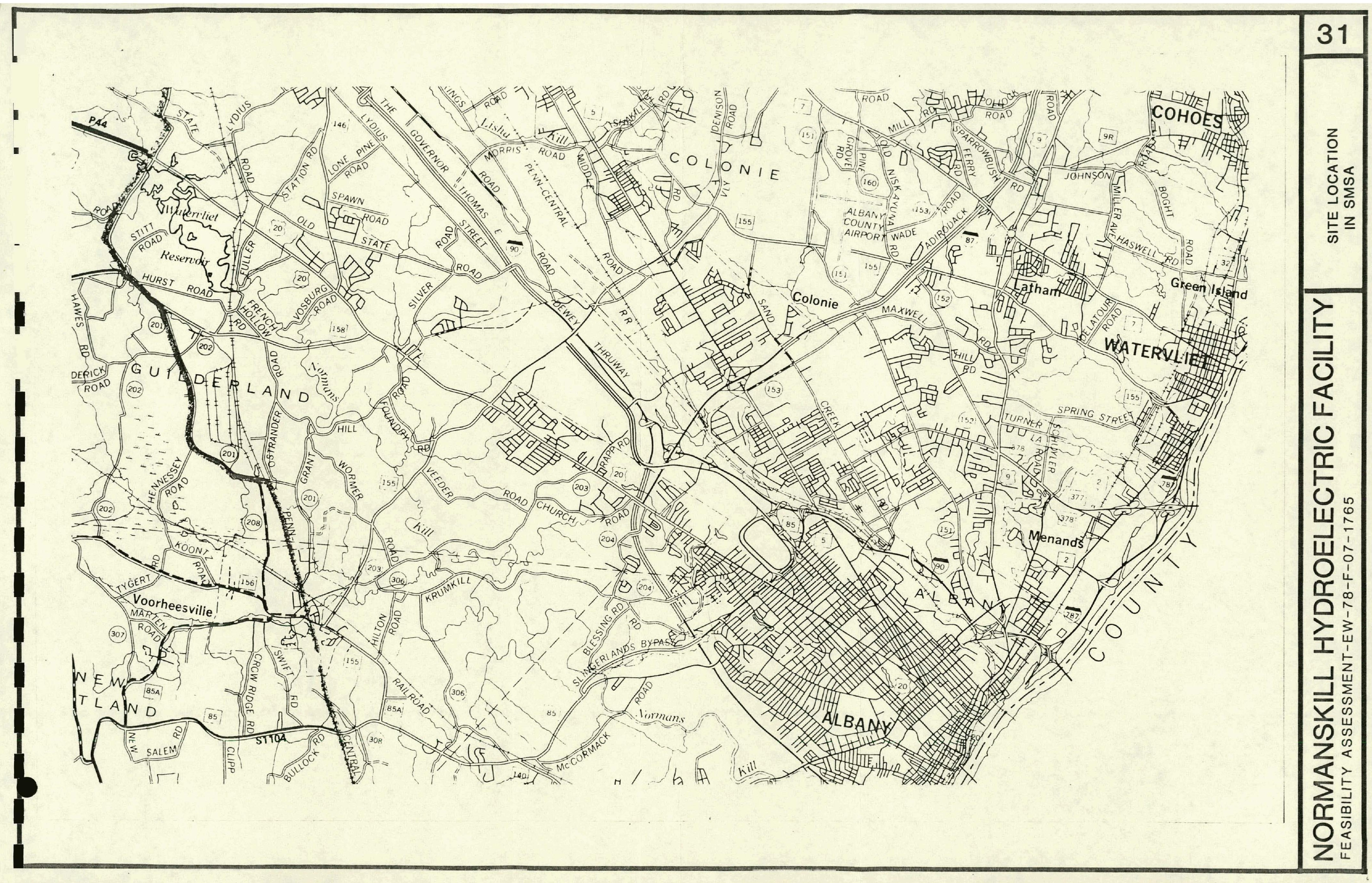




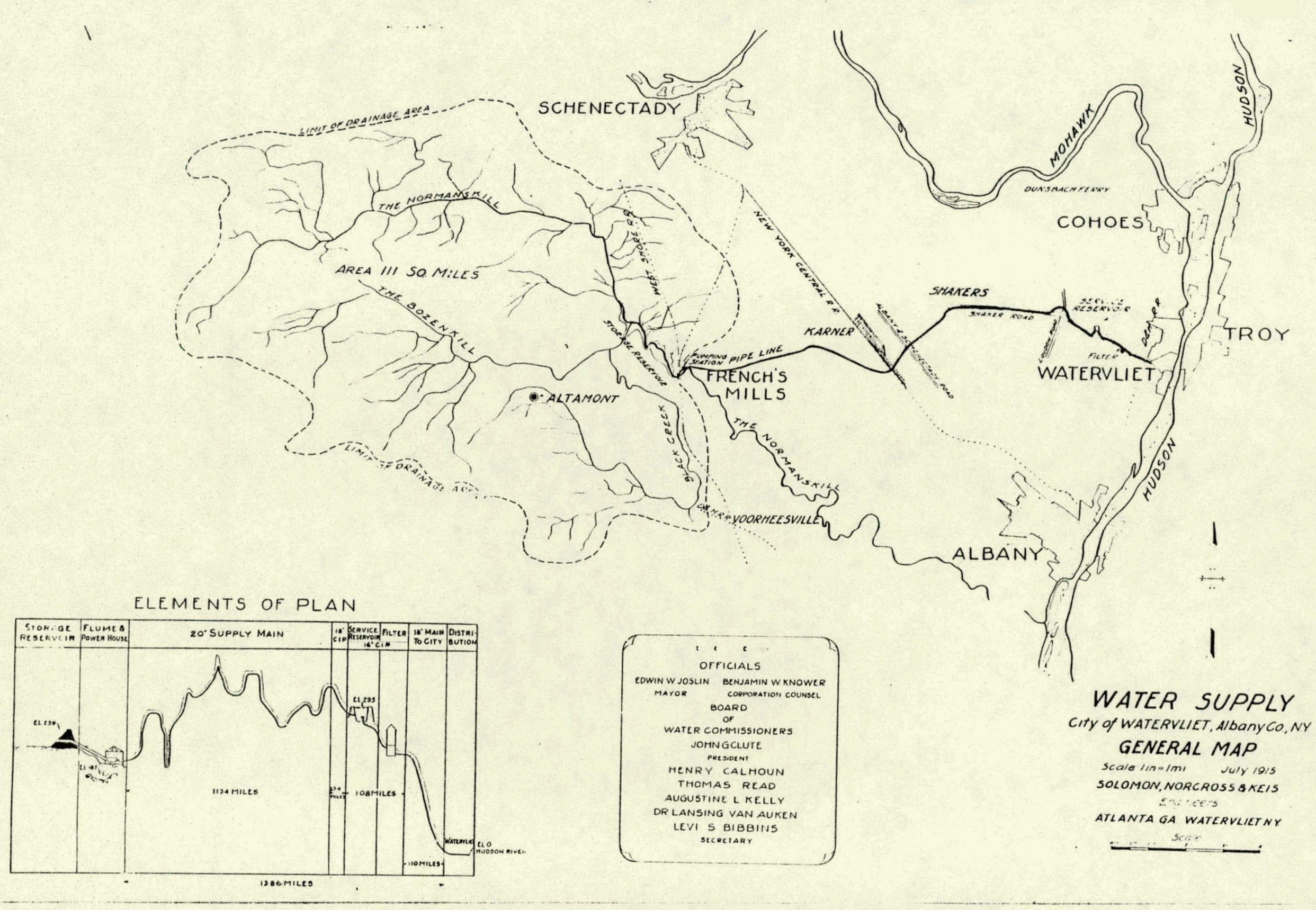




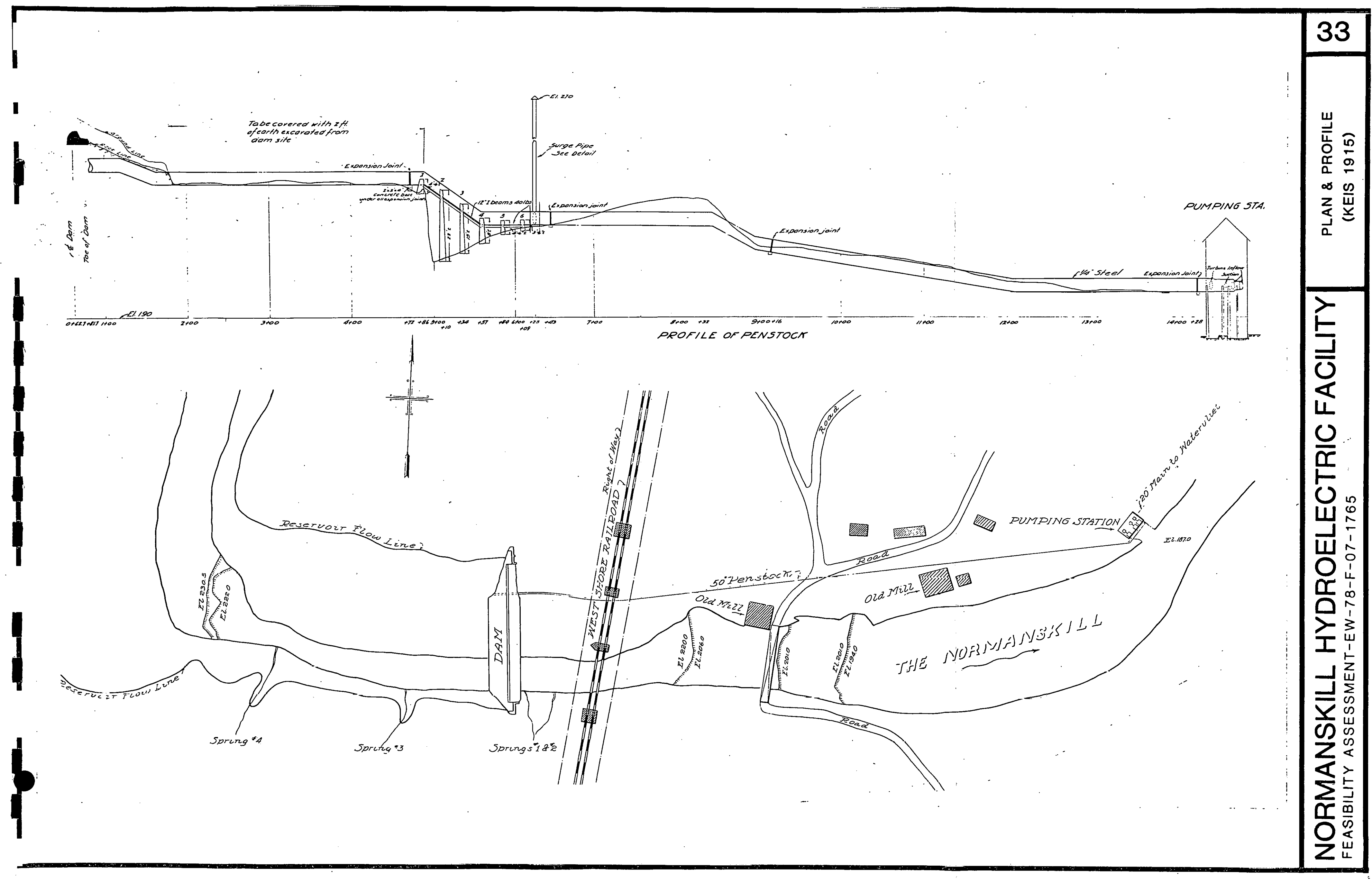




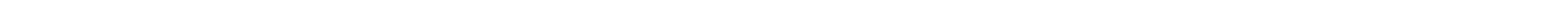





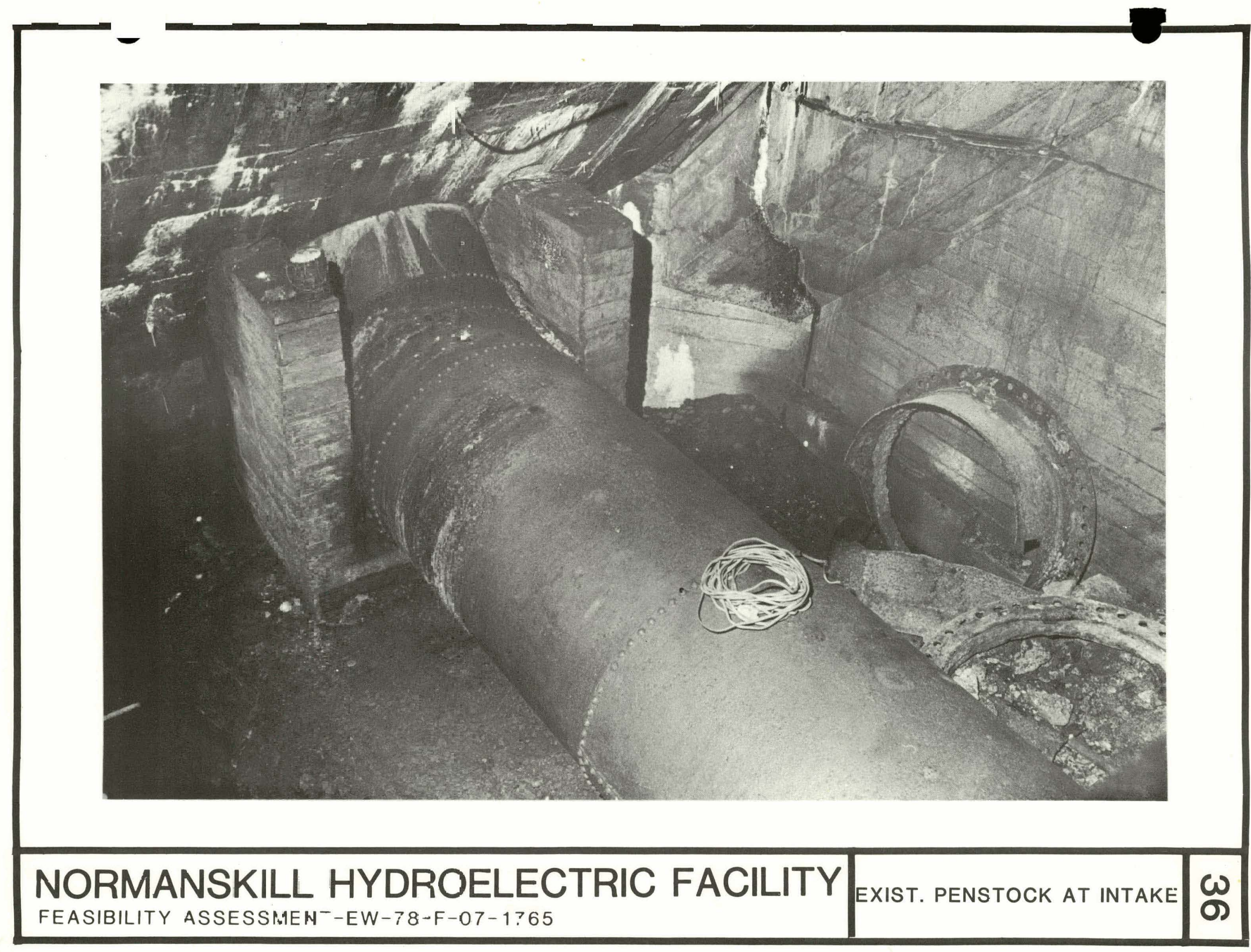




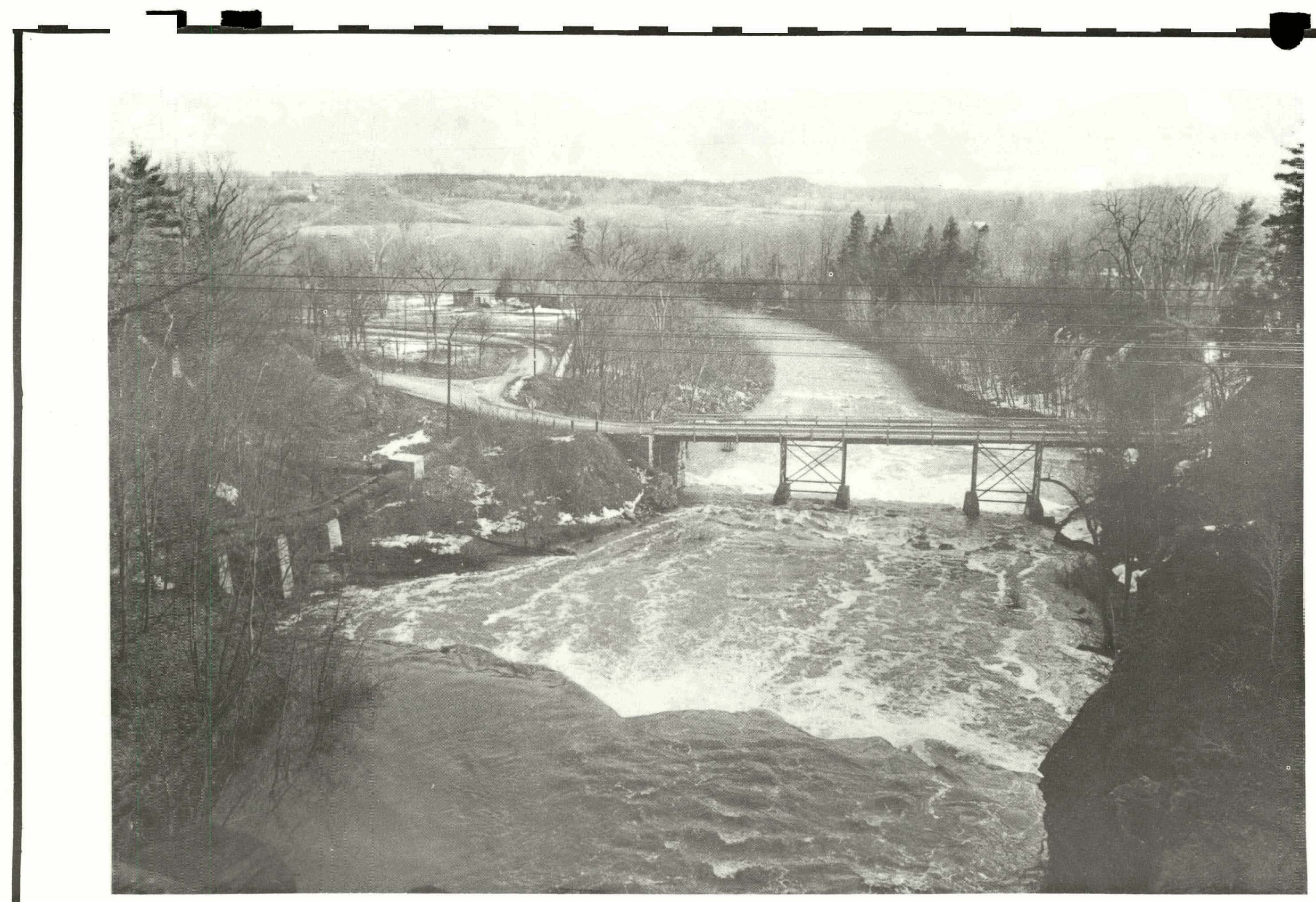




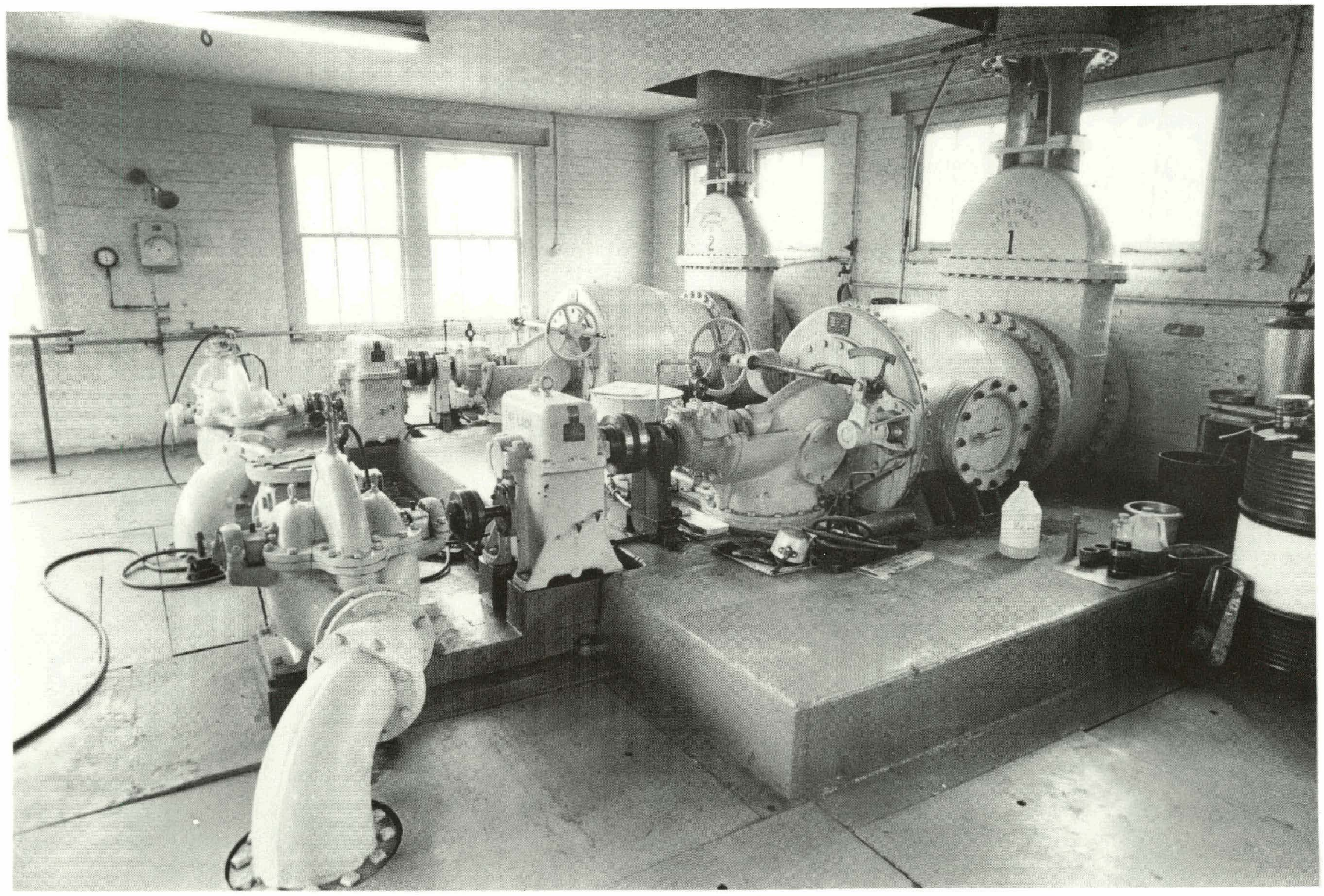




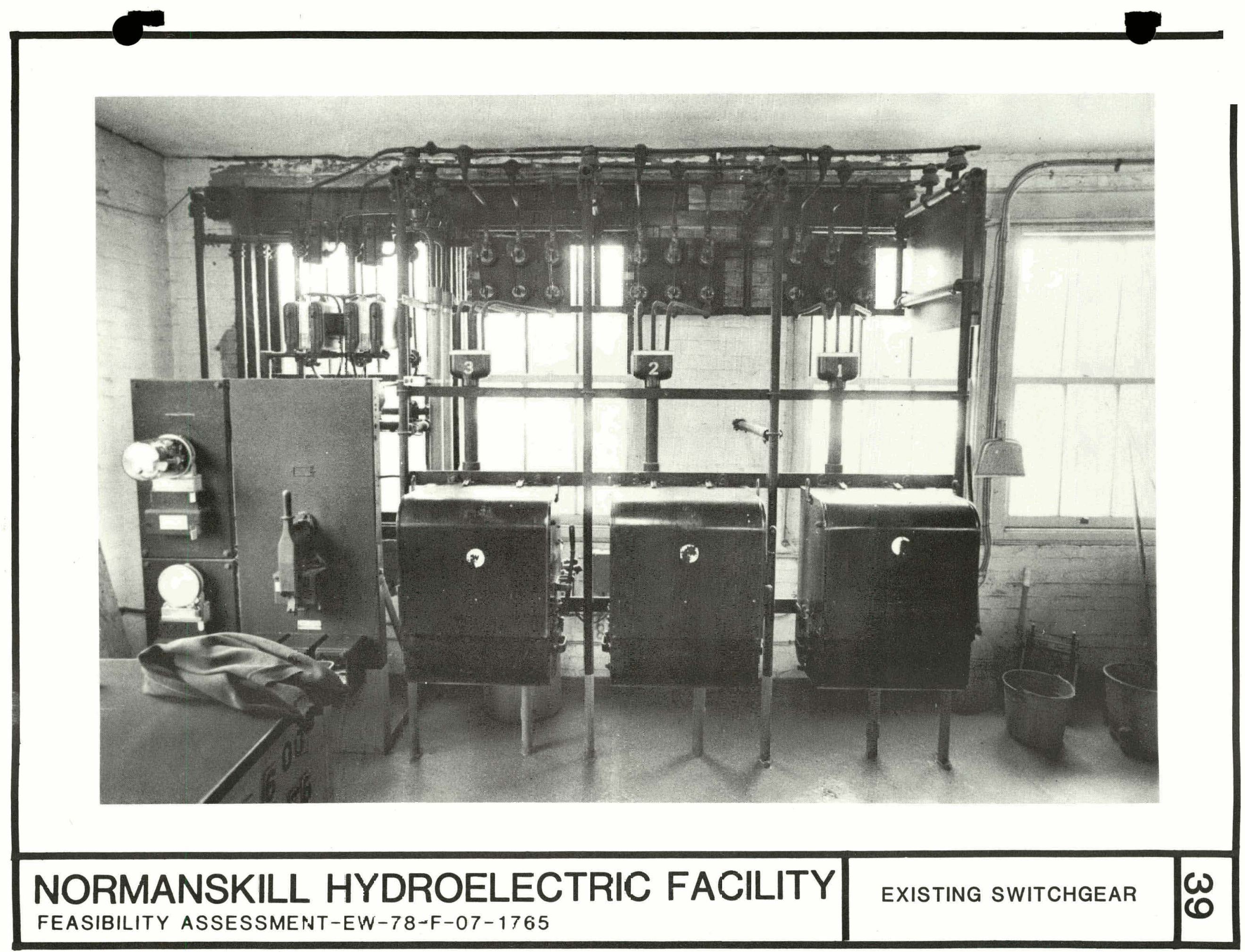




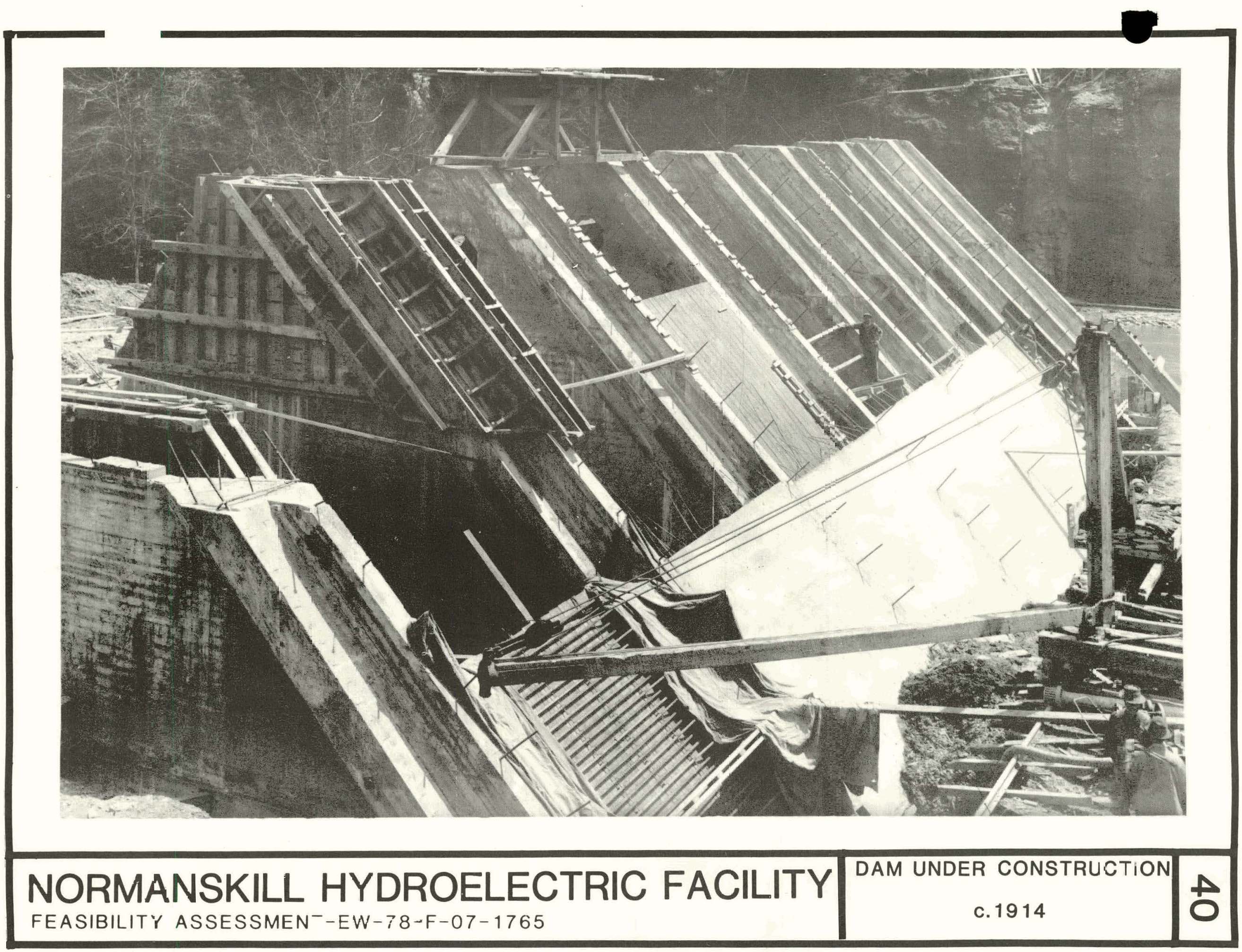




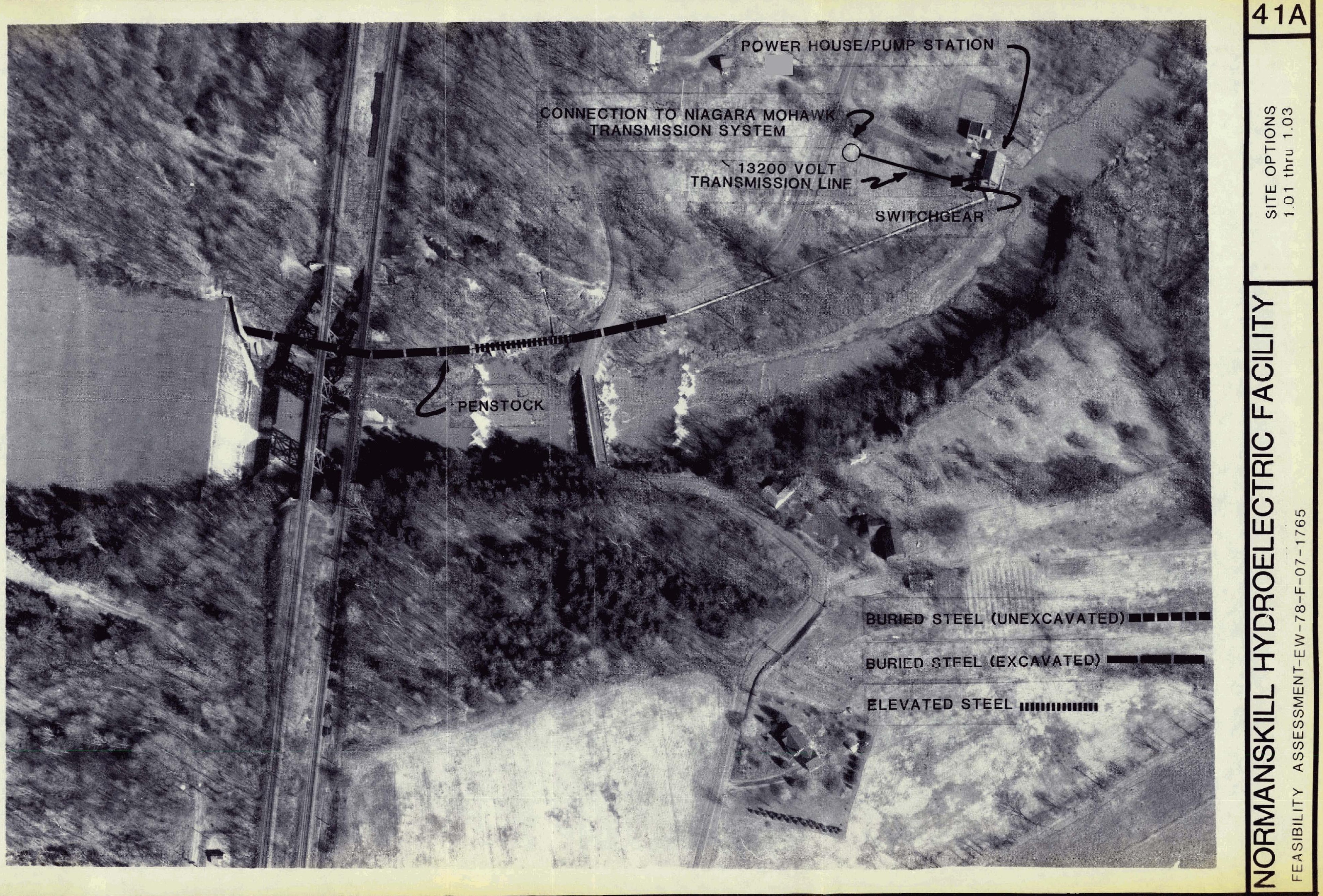




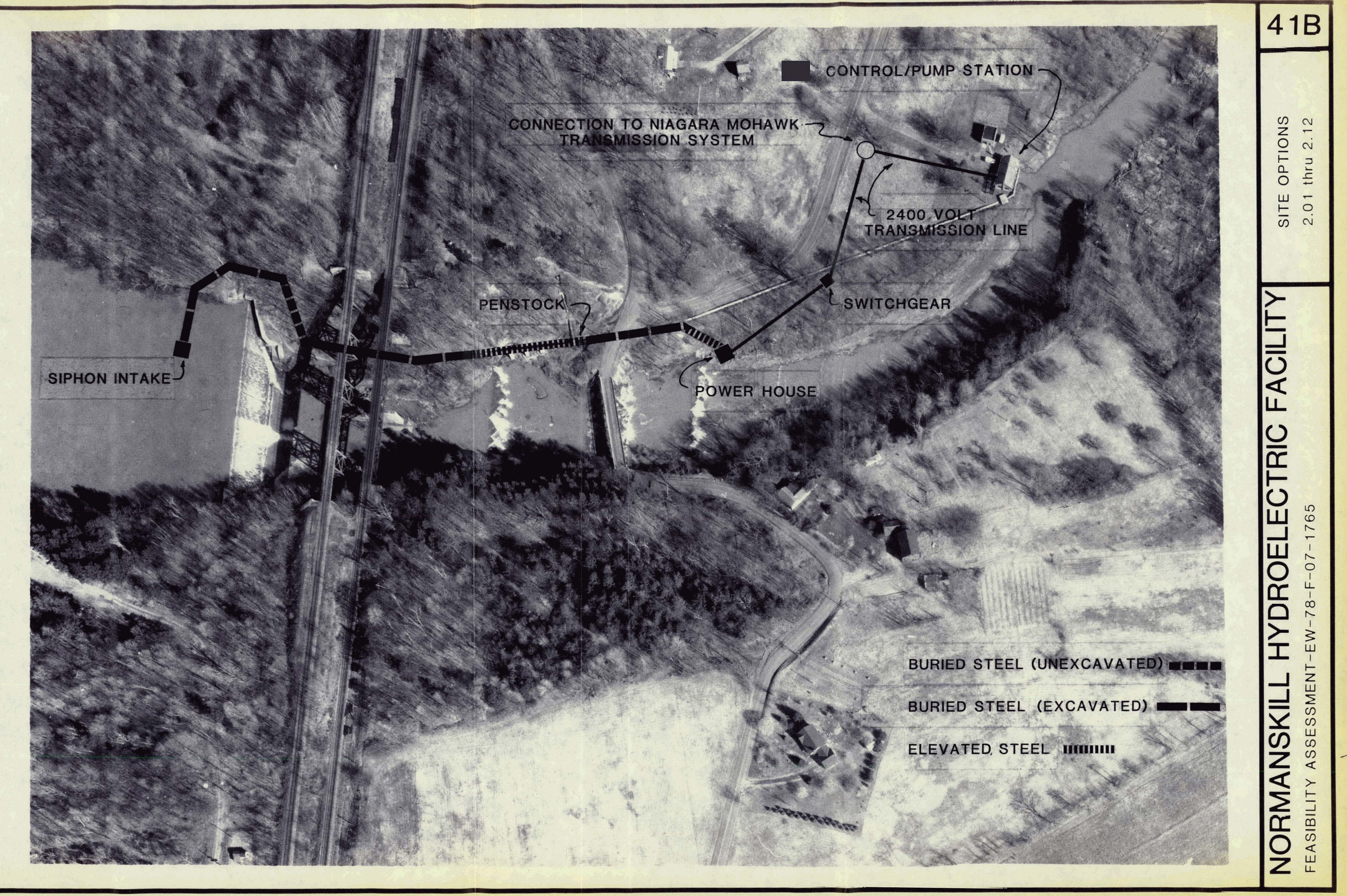




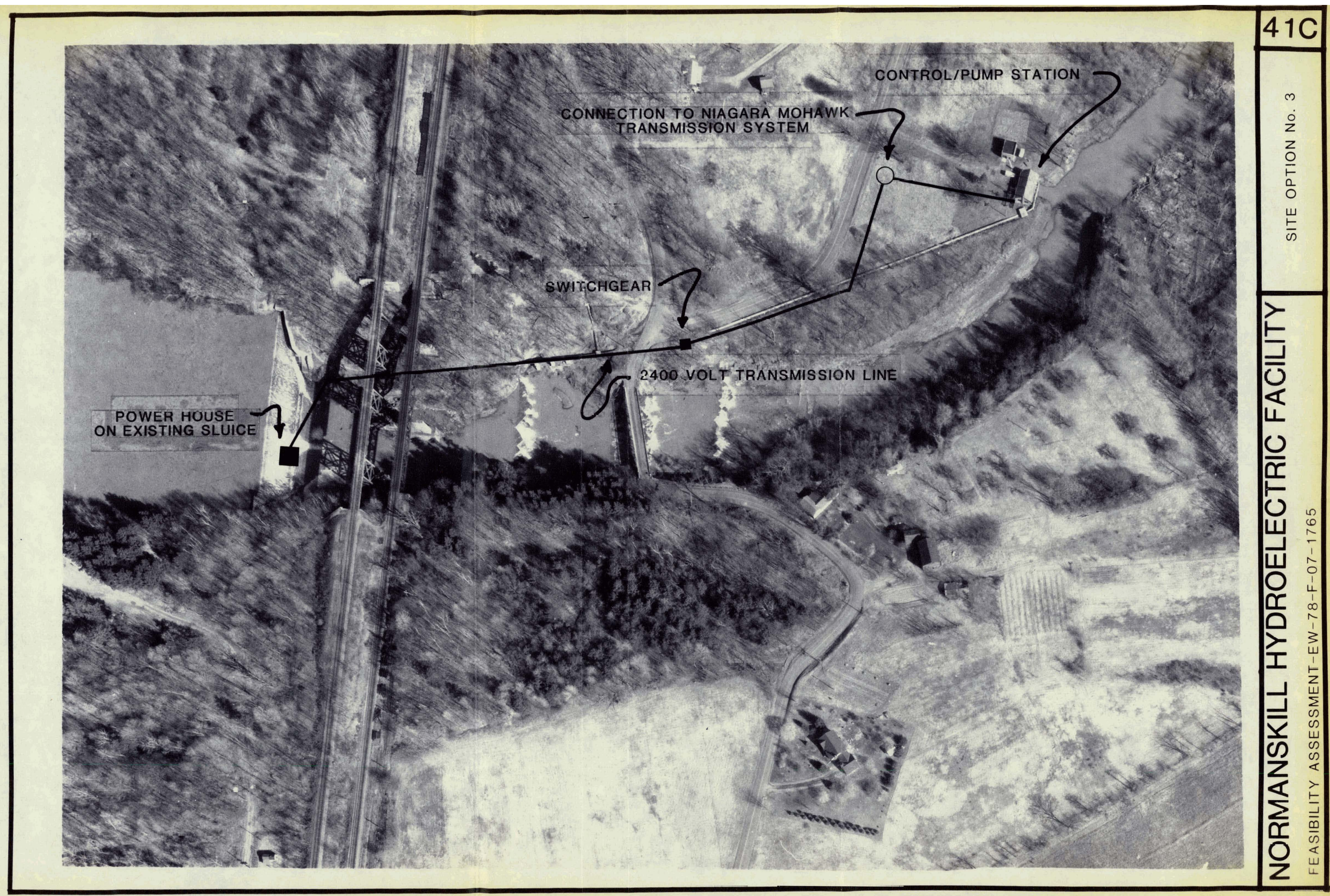




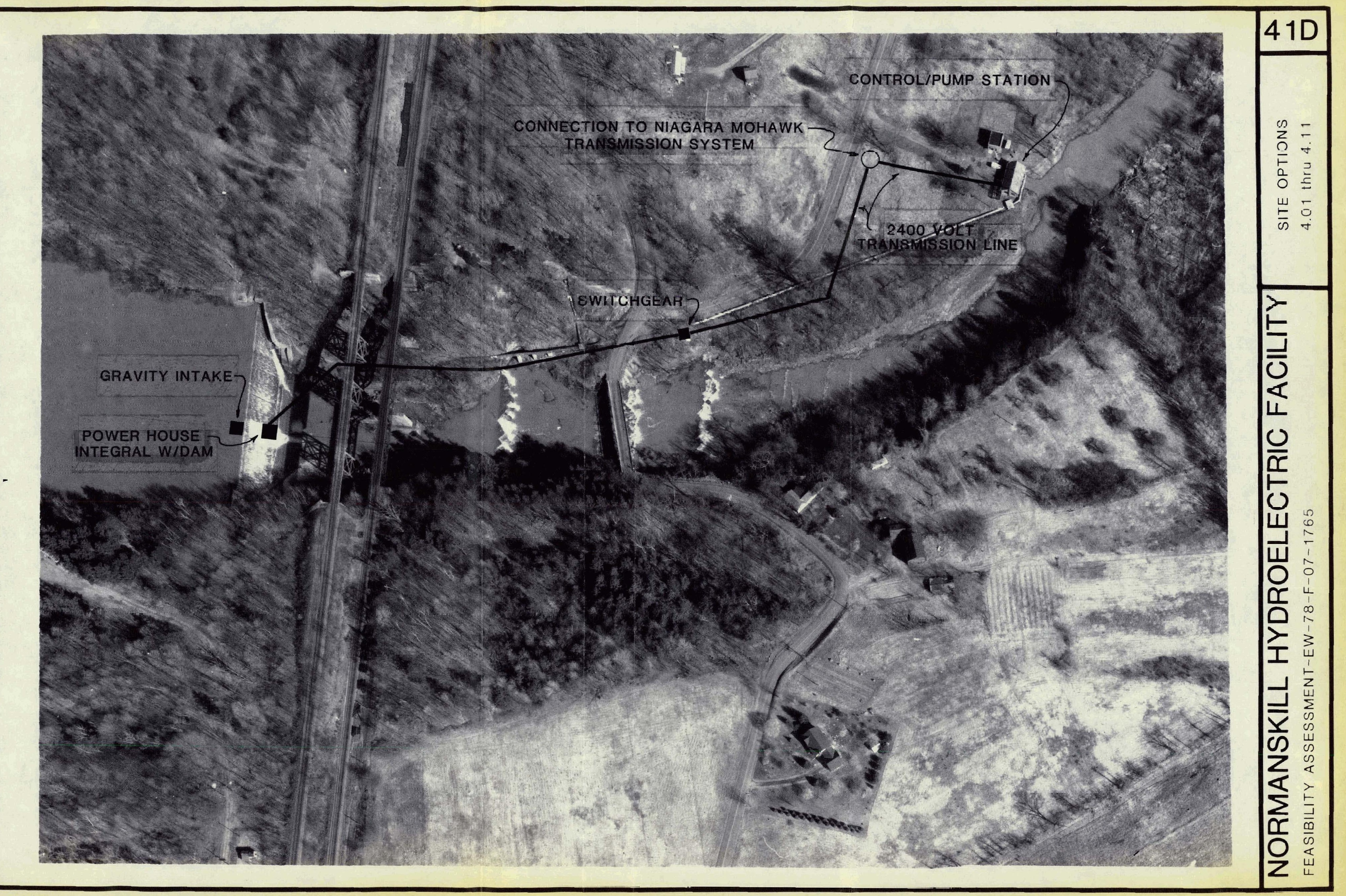




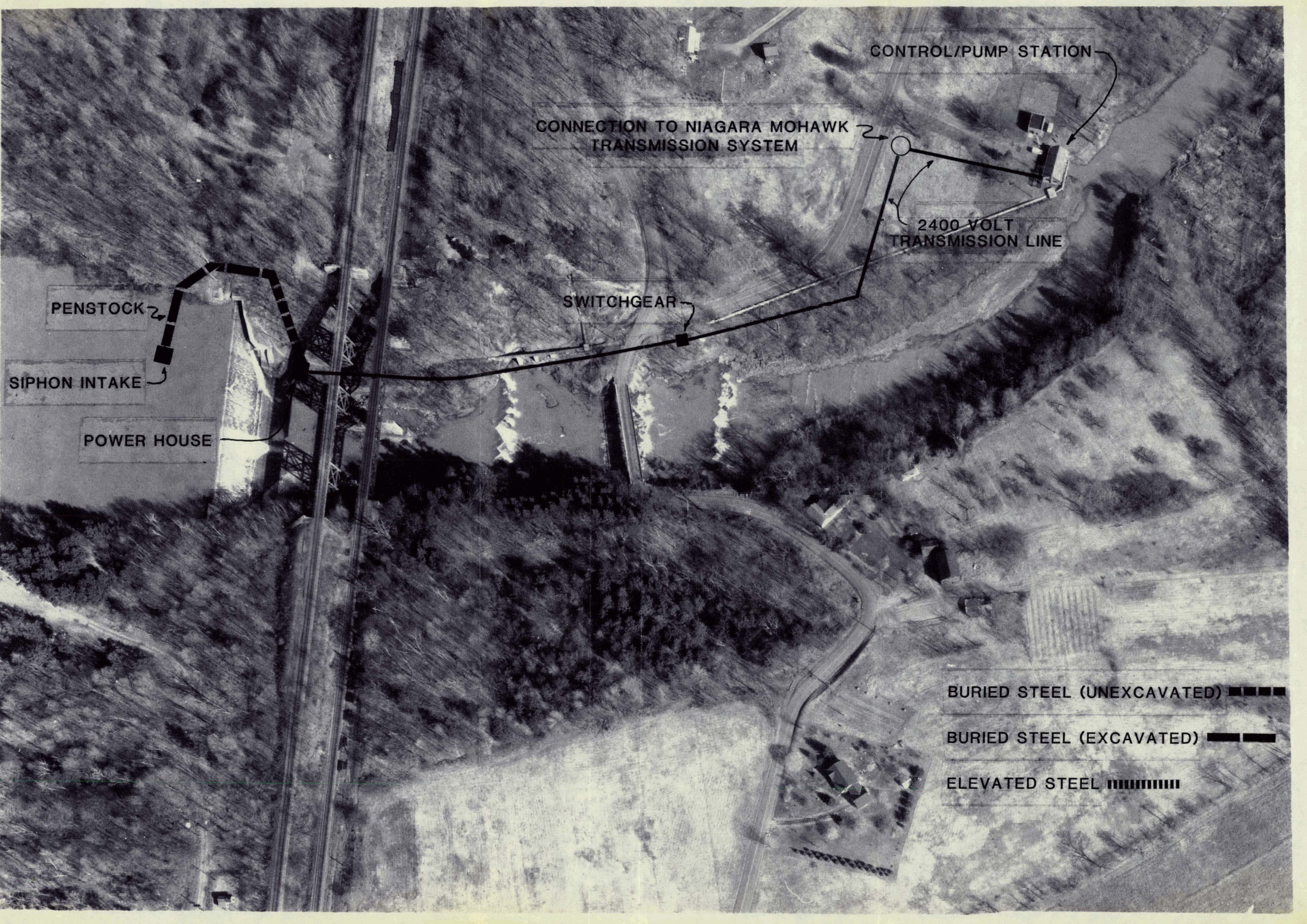




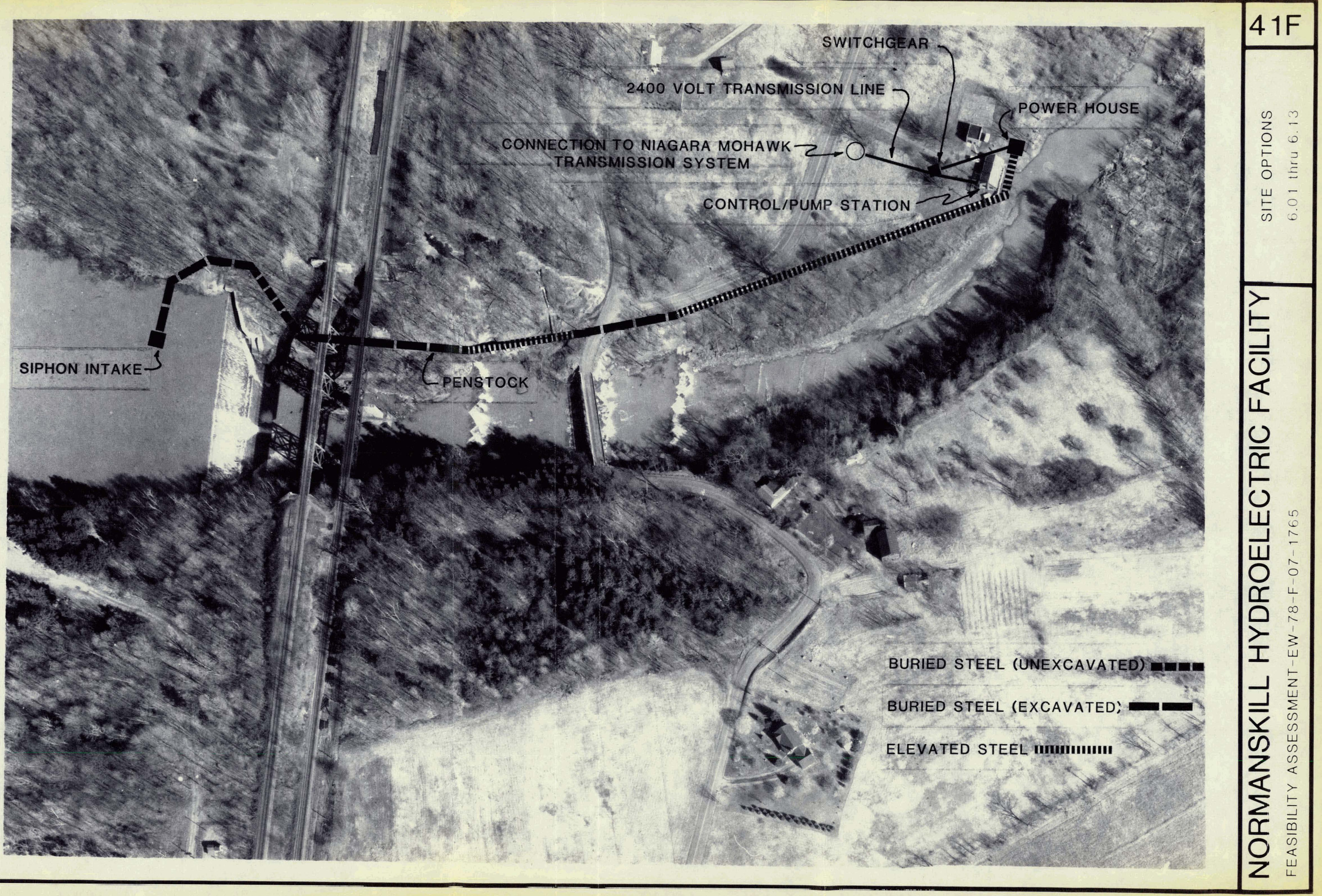




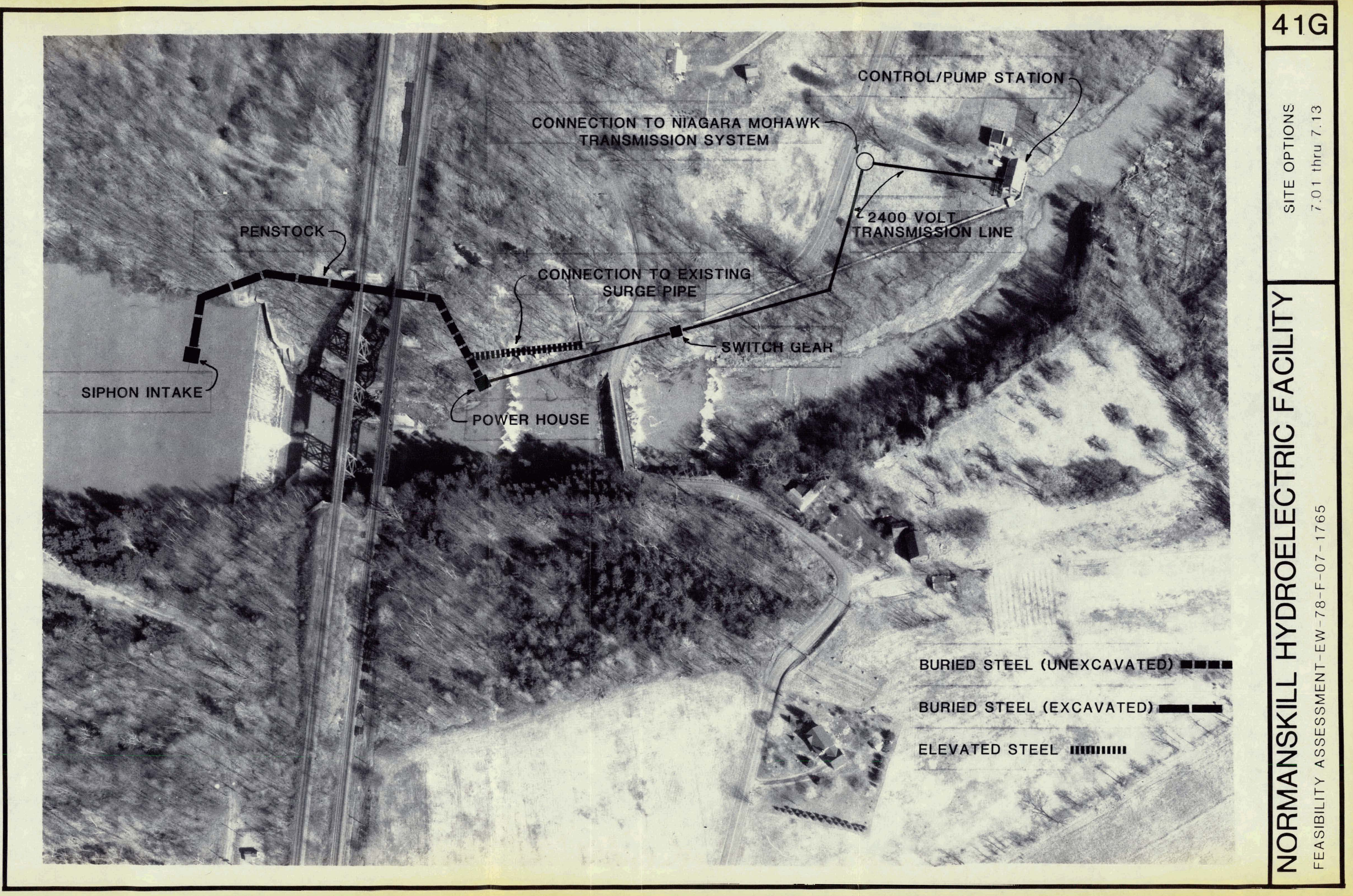




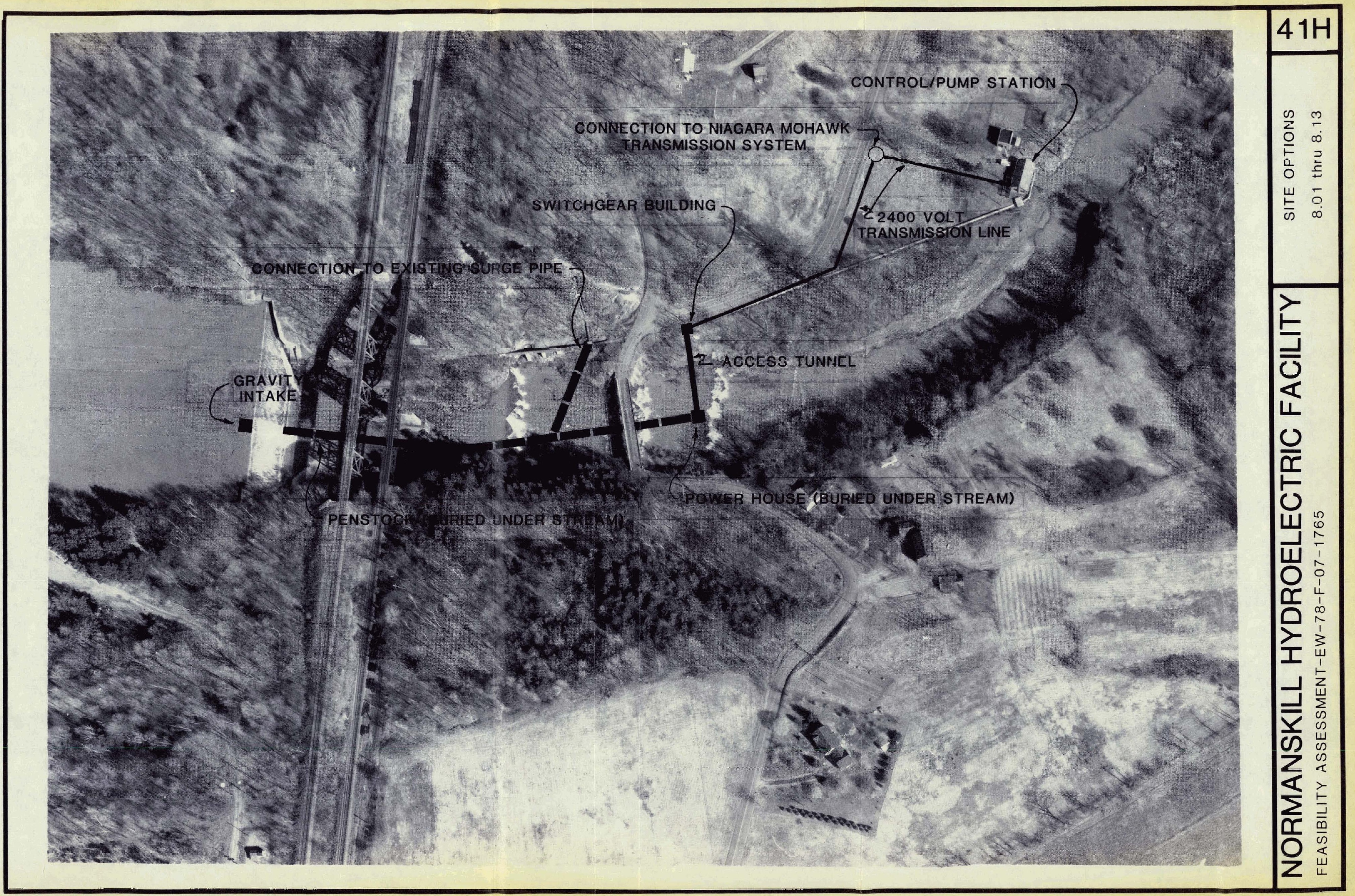


$A L t$

EXCAVATION IN ROCK

42

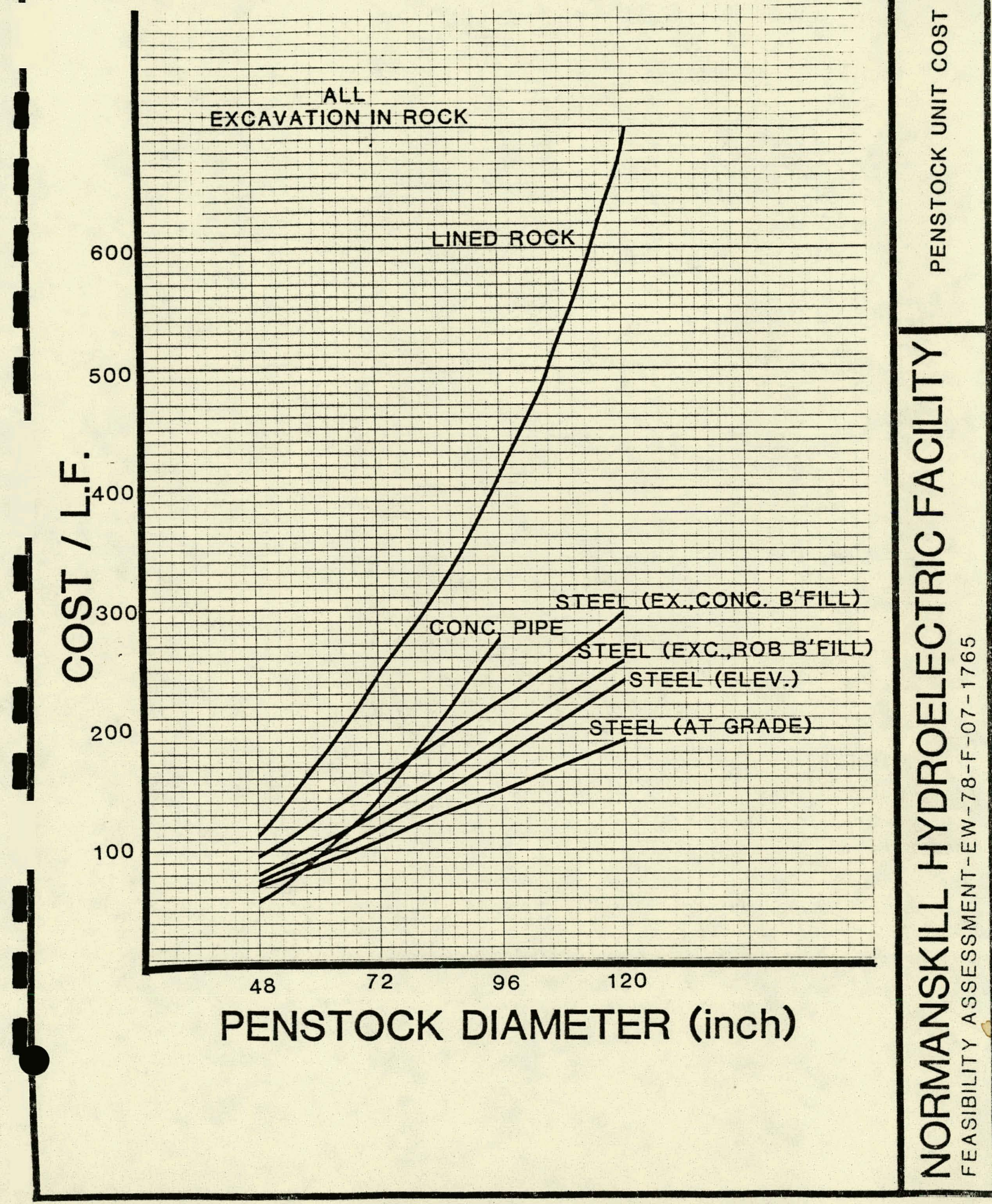




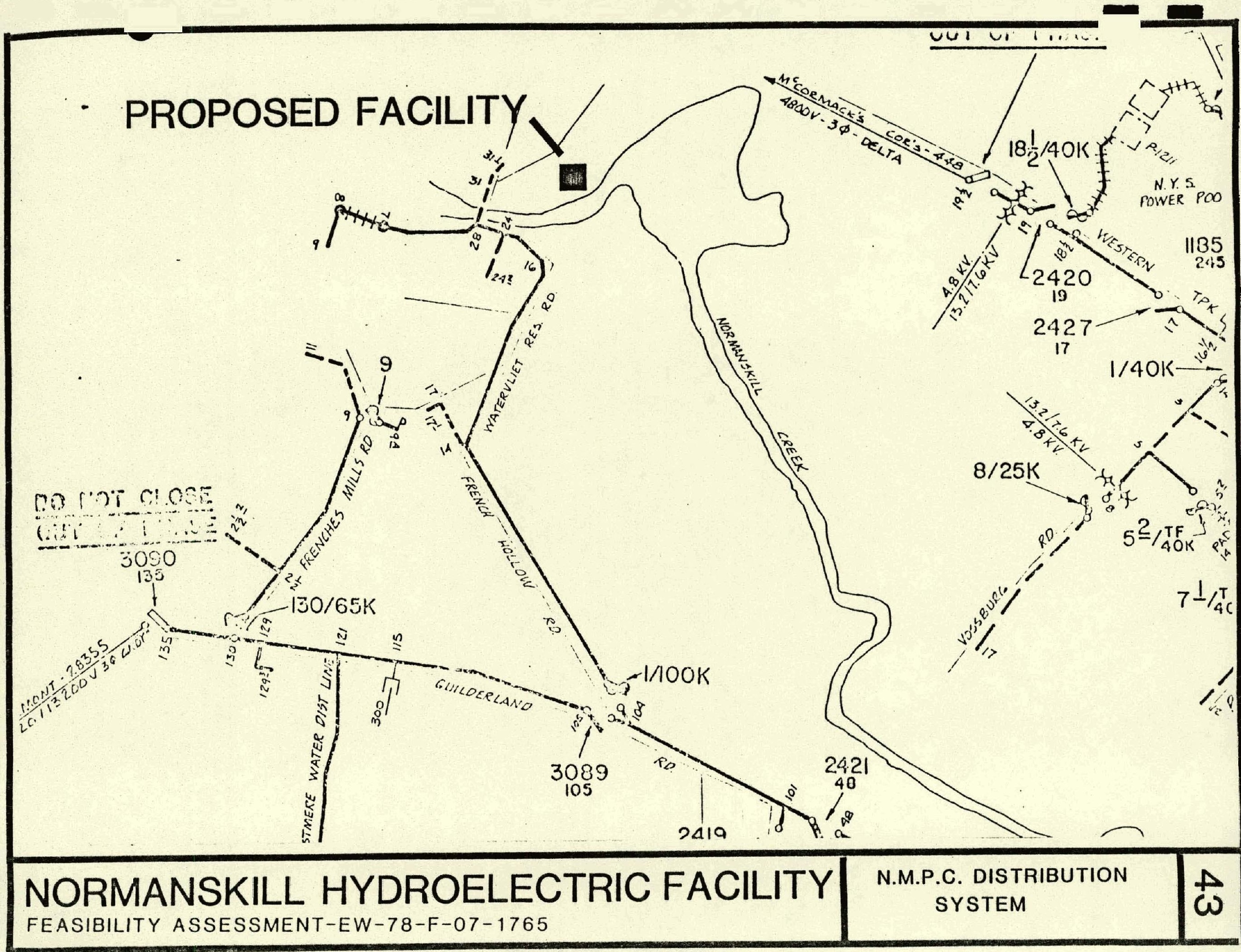




\title{
OPTIMUM CONFIGURATION
}

\author{
ALTERNATES $1.01-8.13$
}

PERIOD

DISCOUNT RATE

O. \& M GRADIENT

ENERGY GRADIENT
50. YEARS

\section{$8.0 \%$}

$4.0 \%$.

$7.0 \%$

MACHINE TYPE:

1 VERT. FRANCIS

2 HORZ. FRANCIS

3 HORZ: PROPELLER(ADJ.)

4 VERT. PROPELLLR(FIXED) 
NURIAANS KILL. HYIIRO-ELECTRIC, FEASJRILITY STUIIY OPTIMIUM CONFIOURATION PROGRAM (OCPHI)

S) YEAR SERVICE PERIOII PRESENT WORTH ANALYSIS

FASEL UPDN SO YEAR EQUIPMENT LIFE ANI G.00D PERCENT IISCOUNT RATE

1. ALTERNATIVE NO.

2. LIESIBN FI.OU

3. GROS! HEAII

4. PENSTOCK DOTAMETER

5. JNTAKE AREA

b. HET IIFAT

7. TURBJWE TYPE

6. RIJNINER IIITMETER

9. GETTING

10. SPECIFIC: SPEEII

1. UNIT EFFJCIENCY Q F.L.

12. COMPOSITE EFFICIENCY

13. NE' POWER

14. PENGTOCK L.ENISTH

15. PENGTOCK COST

1.6. INTAKE COST

17. POWEPHOUSE COST

19. SWITCHGEAR COST

19. MACIIINE COST

20. ANC. EQUIP. COST

2.. MOHIL. \& TEMP. SERV, COST

22. ALIMIN., LECGAL. \& TECH. COST

23. INT . WURING CONSTRUICTION

24. TOTAL CAPJTAL COST

(CFS)
(FEET)
(INCHES)
(SQ.FT )
(FEET)
(INCHES)
(FEET)

1.01

6.55

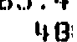

132

60.7

2

12.7

9.19
73.14

73.14

78.0

(\%)

25. P.W. OF CAPITAL COSTS

(KW) 136

1500

(\$)

68400
537010

$537010 \quad 69400 \quad 68400$

$42800 \quad 53700 \quad 53700$

$42800 \quad 91200 \quad 91200$

$\begin{array}{rrrr}(\$) & 194200 & 201500 & 201500 \\ (\$) & 107900 & 257500 & 259900\end{array}$

$\begin{array}{rrrr}(\$) & 107900 & 257500 & 259900 \\ \text { (\$) } & 70300 & 70300 & 70300\end{array}$

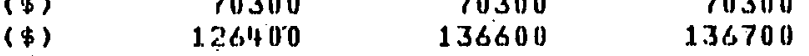

$\begin{array}{rrrr}(\$) & 126100 & 136600 & 136700 \\ (\$) & 113100 & 131300 & 130400 \\ (\$) & 31100 & 40400 & 40100\end{array}$

(\$) $\quad 807900 \quad 1049900 \quad 1051500$

(\$) $807900 \therefore 11149900 \quad 1051500$

26. BASE YEAR ANNUAL O \& M COST(\$) 27. $0 \& M$ CMPI. COST GRALIENT (\%)

28. P.W. OF SO YEAR O \& M COST (\$)

807900

051500

B18400. 997100

29. P.w. OF c0sTs $[25 \& 20] \quad$ (\$) $2626300 \quad 20147000$. 2050900

30. HASE VALTJE/YR ENHERGY GEN'II. (\$)

31. ENERGY C.MPIH, VALUE BRAIIENT $(\%)$

32. P.W. OF SO YEARS OF ENERGY (\$)

53900

$09500 \quad 09500$

$7.0 \quad 7.0$

33. H.W. UF RESTIHAL VALUE (\$)

$2145100 \quad 3561900 \quad 3561900$

34. P.W. OF CREITTS $332 \& 33]$ (\$) 2145100 . $3561900 \quad 3561900$

35. P.W. OF NET C.OST [29-3it] (\$)

$-1514900 \quad-1511100$

36. RANKJHG WYY LEAST NET COST P.W.

3 
NORMANS KILLL HYLRO-ELECTRIC FEAGIUILITY GTUIY

OPTIMUM CONFIGURATION PROGRAM (OCPHI)

50 YEAR SERVICE PERIOD PRESENT WORTHI ANALYGIS

FASEI UPON SO YEAR EQUIPMENT LIFE ANII G.000 PERCENT DISCOUNT RATE

1. ALTERNATIVE HD.

2. DESIGN FI.ONS

3. GROSS HEALI

4. PEHSTOCK DITAMETER

5. INIAKE AREA

b. HET HEAII

7. TURBINE TYPE

B. RIINNEER IUIAREETER

9. SETTING

10. BPECIFTC SPEEH

11. UNIT EFFICIENCY A F.L

12. COMPOSTIE EFFICIENCY

13. NET POWER

14. PENSTOCK LENIGTH

15. PENSTOCK COST

16. INTAKE COC:

17. POWERHOUSE COST

18. SNITCHGEAR COST

19. MACHINE COST

20. ANC: EQUJP, COST

21. MOHIL. \& TEMP. SERV, COST

22. AlIMIN. , LEGAL \& TECH, COST

23. INT RIURING CONGTRUICTION

24. TOTAL CAPITAL. COET

25. P.W. OF. CAPITAL COSTS

(CFS)
(FEET)
(INCHES)
(SQ.FT.)
(FEET)
(INCHES)
(FEET)

2.01

100

2.02

84
48

50

59.3

27.3

8. 4

(\%) $\quad 74.9$

(\%) 82.9

$\begin{array}{rr}(\%) & 82.11 \\ (\%) & 78.3 \\ \text { (IW) } & 386 \\ \text { (FEET) } & 900\end{array}$

100
64.4

6.4
60

60
50

62.1

$27 . \frac{1}{3}$

9.5

72.6

由2. 0

78.3

73.3

411
900

(\$) 65600

85500

(\$) $78900 \quad 78900$

(\$) 289700.2911700

(\$) $275200 \quad 288100$

(\$) 70300

139600

$162700 \quad 165300$

60300

(\$) 58700

(\$) 1527100

(\$) 15271001566800

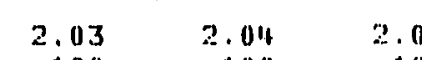

64.14

64.14
72
50

50

63.3

27.1

日. 9

71.9

62.0

78.3

119
900

$108300 \quad 134000$

386100

70000

78000

291200
301700

$\begin{array}{rr}295500 & 301700 \\ 70300 & 70300\end{array}$

$1+1100 \quad 142700$

167600

$161600-1300$

63000

$16016001639100 \quad 1674200$

$1601600 \quad 16391001674200$

16391001674200

$2.05 \quad 2.06$

100

96

50

64,0

27. 1

日. 6

71.6

70.3

78.3 901

380100

78000

291300

307300
70300

2.06
201

6.14.4

6,10
100

100
55.3

39.1

9.4

76.8

82. 0

80.7

755

05500

$406800 \quad 408300$

$128000 \quad 124500$

$\begin{array}{rr}302500 & 403600 \\ 70300 & 70300\end{array}$

$\begin{array}{rr}70300 & 70300 \\ 140400 & 150500\end{array}$

50500
82100

$\begin{array}{rr}60200 & 70100\end{array}$

17736001823500

$\begin{array}{ll}1773600 & 1823500 \\ 1773600 & 1823500\end{array}$

$.07 \quad 2.08$

$2.00 \quad 2.09$

$2.09 \quad 2.10$

4.11

100

60.4

30.3
9.2

73.6

02.0

B25

64.4
76

100

63.2

1
38.4

9.1

72. 0

82.0

80.

863
900

158201

408800

408800

306500

422600

70300

154100

187400
73300

73300

1906100

64.1

72

150

56.0

47.9

10.0

76.3

ต2. 0

75,3

$1.3 \quad 75$.

1149

$100300 \quad 134000$

$426400 \quad 426400$

$169300 \quad 165000$

$312600 \quad 314700$

$455800 \quad 476200$

$70300 \quad 70300$

$156600 \quad 158600$

91200
75600

$965100 \quad 2017500$

26. HASE YEAR ANNUAL O \& M COST(\$)

27. O. \& M CMPH, COST GRADIENT (\%)

5640.10

57700

60200611100

6.650

69300
4.0 $71300 \quad 73100$

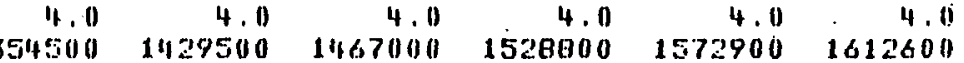

124420012729001299400.132800013545001429500 1467000 152860015729001612600

(\$) $2771300 \quad 2839700 \quad 2901000 \quad 2967100 \quad 3028700$

$3203100 \quad 3290500$

34311900

29. P.W. of COSTS [25 \& 23] (\$)

30. HAGE VALUE/YR ENERGY GEN $[1,(\$)$

$97200-100300$

$101200 \quad 101500$

111500

$113900100500 \quad 112500$

31. ENERGY CMPH, VALIJE GRAIIJENT

33. P.W: OF RESTIMUAL VALUE (\$)

3868300

$\begin{array}{rrr}7.0 & 101200 & 101500\end{array}$

17011
7.0

$\begin{array}{rr}7.0 & 111500 \\ 7.0\end{array}$

$3900100500 \quad 112500$

\begin{tabular}{|c|c|}
\hline $\begin{array}{r}7.0 \\
7500\end{array}$ & 7. \\
\hline
\end{tabular}

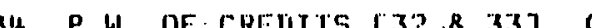

4027500

0

o

o

$\mathbf{0}$

35. P.W. OF NET COST $229 \cdots 34.7$ (\$)

4039500

$401+74$

1453290043180004477200

a 11

16

19

21

$a 11$

15

17
17

-11 1100

! 36

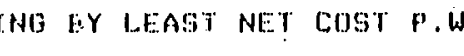

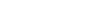

11

.


HORMANS KILL HYIRO-ELECTRIC FEASIEILITY STULY

OPTIHUM CONF T.GURATION PROGRAM (OCPHZ)

50 YEAR SERVICE PERIOI PRESENT WORTH ANALYGIS

HASEL LIPON 50 YEAR EQUIPHENT LIFE ANII 9.0OO PERCENT DISCOUHT RATE

1. NI.TERNATIVE NO.

2. liESICH FLOW

3. BROSS HEALI.

4. PENSTOCK DTALETER

i. INTAKE: AREA

6. NET HE:Ali

7. TURHINE: TYPE

8. RIINNER DIAMETER

7. SETTING

10. SPECTFIC SPEED

11. UNIT EFFIEIENCYY O F.L

12. COMPOSITE FFFICIENCY

13. NET POWER

it. PENSTOCK LENGTH

\title{
15. PENSTOCK COST
}

16: INTAKE COST

17. POWERHOUSE COST

10. SWITCHLEFR CoST

1.9. MACHINE COST

20. ANC. EQUIP. COST

21. MOEIL. R TEMP, SERV, COST

22. ALMIN., LEGAL \& TECH. COST

23. INT MUURLNG CONSTRULTTION

24. TOTAL CAPITAL COST

25. P.W. OF CAPTTAL. Costs

(CFS)
(FEET)
(TNCHES)
(SQ.FT. )
(FEET)
(INCHES)
(FEET)

2.1.1

300

64.4

96

150
62.0

47.3

9.7

72.7

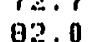

75.3

1185

900

(FEET)

26. EASE YEAR ANHIJAL. O \& $M \operatorname{COST}(\$)$

27. O \& M CMPU, COGT GRAIIENT (\%)

213. P.W. OF 5O YEAR O \& M COST (\$)

29. P.W. UF rosits $[25 \& 28]$

31. HASE VALIIE/YR ENERIGY GEN' D. (\$)

31. ENERGY CMPD. VAL.UE GRAIIENT (\%)

32. P.W. OF 50 YEARS OF ENERGY (\$)

114400

$114+00$

4552700

50200

165500

315700

409500

70300

1973011

79301

2061901

2061900

74600

4.0

2.12

5il)

64.4
96

96
250

59.1

61.1

10. 2

74.4

Q2.0

$71+3$

1958
900

158200

158200
+57000

457000

233800
332300

601700

70300

172200

214200

89600

2329300

2329301

4. 01

100
35.4

35.4
4.65

50

34.3

24.9

1.0

151.6

日1.0

Q1. 3

236

2411

3300

3.300
261400

264400

300000

31.3300

81300

70300

536011

149200
51100

1325200
1325200

1326400

4.0

49500

$\begin{array}{rrr}4.0 & 4.0 & 4.0\end{array}$

49900
$4094200 \quad 1100900$

1100000

2435300

2611000

114700

2417200

242260

69500
7.10

70500
7.0

71.00
7.0

34100
7.0

7.0
347000

$+564800$

2829600

33. P.W. OF RESILIUAL. VALLUE

(\$)

0

0

1)

0

()

)

0

0

10

(1)

\begin{abstract}
0
\end{abstract}
$-1940.2010$

39900

$-34670$

36310

36. RANKJHG BY LEAST NET COST P.W.

26

5

58

394.300

$\frac{3.347000}{-736200}$

20

339080

$3406700 \quad 3416600 \quad 3492300$

5

58

411

311

3

706800 -716400 
NORMANG KILL. 'HYIURO-ELECTRIC FEASTHILITY STULY

OPTIMUIA CONFTGURATIOA: PROGRAM (OCPHI)

50 YEAR SERVICE PERION PRESENT WORTH ANALYSIS

EASEII UPON SO YEAR EQUTPMENT LIFE ANII G.00O PEECENT VISCOUNT RATE

1. ALTERNATTVE TO.

2. TIESIGN FLOJW

3. BROSES HEAII

4. PENSTOCI IITAIAETER

5. IITAAKE ARE:A

6. NET HEAD

7. TURHTNE TYPE

日. RUNHER DIAMETER

9. SETTING

J. SPECIFIC SPEEL

1. WWIT EFFICIENCY O F.L.

12. COMPOSITE EFFICIETCY

13. NET POWER

14. PENGTOCK L.ENGTH

15. PENSTOCK COST

16. INTAKE COST

17. POLERHOUSE COST

18. SWTTCHGEAR COST

19. MACHINE COST

20. ANC. EQUIP. COST

21. MOYIL. \& TEMF. SERV. COST

2. AlHIN, I.ELAL \& TEC.H, COST (\$)

23. INT DUURING CONSTRIJCTION

24. TOTAL. CAPITAL COST

25. P.W. OF CAFITAL COSTS

(NFG)
(FEET)
(INCHES)
(SA.FT.)
(FEET)
(INCHES)
(FEET)

4.07

30

84

150

34.4

3
43.1

43.1
-1.5

154.4

Q1.0

01. 7

(\%)

(FET)

713

4.10

300
35.4

96

150

34.8

43.11

$-1.7$

153.7

ย1. 0

01. 7

722
50

(\$)

7.100

297100

8300

287801

308900

36000

(\$) 308600

(\$) 362600

(\$) 209001

(\$) 70300

211500

70300

62500

62300

162900

163100

59000

(\$) 56900

(\$) 1530500

1534100

$1.53+100$

$\begin{array}{ll}1671600 \\ 0 & 1671600\end{array}$

4. 1.1

500
35.4

96

33.9

33.9

55.6

$-1.6$

155.3

ด1.0

62.2

1179

8300

300200

308000

376300

294900

70300

68300

172200

64300

56600

4.0
+3600

61300

1246400

2776900

270270

$302390(1)$

08600

09200

70000
7.0

30. EASE VALIJE/YR ENERGY GEN I. (\$)

7.0

$3526100351+9900$

3613600

5.01

100
35.4

it

50

31.1

29.6

10.4

99.0

62. 1

78,3

206 401

31400

388100

03900

312900

205500

70300

105600

154600

406400
1406400

14064011

5.112

100
35.4

60

337

2日. 1

11.0

95.8

62.0

79.3

223

43701

43700100
0.010

92700

313900

217800

70300

106000

156600

55200

1435100

1435100

5.03

500
355.4
70

72

34.5

1

28.3

9.9

94.9

82. 0

70.3

229

231

55100

388100

Q2700

225100

70300

107800

158100

56100

1457500

1457500

.5.04

35.11

104

3150

1

28.3

9.7

94.4

62.

231 100

66500

368100

027011

314400

231200

70300

108700

159400

56900

1476200

1478200

$\begin{array}{rr}52300^{\circ} & 53300 \\ 4.0 & 4.0\end{array}$

54000

5470

$\begin{array}{rrrrrrr}52300 & 53300 & 54000 & 54700 & 55500 & 59300 & 60400 \\ 4.0 & 4.0 & 4.0 & 4.0 & 4.0 & 4.0 & 4.0 \\ 1153800 & 1175800 & 1191300 & 1206700 & 1224400 & 1308200 & 1332400\end{array}$

25602002610900

$2689+90$

272490

2977000

60700

653010

6670

66710

6730

7.3010

67501

76200

$7.0 \quad 80800$

32. P.W. OF SO YEARS OF RENERGY (\$)

()

()

()

0

598600

7.01
$651+5010$

7.0
2629400

$\begin{array}{rrr}7.0 & 7.0 & 7.0 \\ 2686300 & 3032600 & 3215600\end{array}$

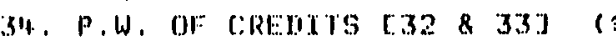

1) 354990

361360

2415700

0

35 . P.W. OF NET cost [29-34] (\$)

74920

$-76720$

$-599700$

14.4500

12100

$-5700$

700

65010

68

65

66

$\begin{array}{ccc}3686300 & 3032600 & 3215600 \\ 38600 & 110100 & -237000\end{array}$

30

35

50

73

68

(n)

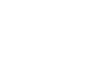

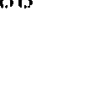

70 $6.11 \quad 4.3$


HORMANS KILL HYLRO-ELECTRIC FEASIBILITY STUMY

OPTTHUM CONFTGURATION PROGRAM (OCPH2)

SO YEAR GERVICE PERTOD PRESENT WORTH ANALYGIS

GASED UPON SO YEAR EQUIPHENT GJFE ANI G. UOU PERCENT DISCOUPT RATE

1. AI.TERHATIVE NO.

2. IUESJON FLOW

3. BROSS IIEALI

4. PENSTOCK IITARETER

5. INTAKE AREA

6. NET HEAL

7. TUREINE TYPE

B. RUNNER DTAMETER

9. SETTING

1.0. SPECIFIC SPEED

1.1. UNIT EFFICIENCY O F,L

12. COHPOSITE EFFTCIENCY

13. NET POHER

1.4. PENSTOCK LENGTH

15. PENSTOCK COST

16. INTAKE COST

17. POWERHOUSE COST

19. SWITCHGEAR COST

19. MACIIINE COST

20. AHC, EQUIP, COST

21. MONIL. \& TEMP. GERV. COST

22. ALIMIN, LEGAL \& TECH, COST

23. INT IURIHE CONSTRUCTION

24. TUTAL CAPITAL COST

25. P.W. OF CAPJTAL COSTS

(C)
OFE
ISQ.F
CFE
SINCH
IFE
CFE
COST
COST
ION

(CFS)

(FEET)

(TNCHES)

SQ.FT.)

(FEET)

IN(CHES)

(FEET)

$(\%)$

(\%)

( $(W)$

(FET)

(\$)

26. WASE YEAR ANNUAL $0 \& M \operatorname{COST}(\$)$ 37. 0 \& $M$ CMPLI COST GRALIENT (\%) 20. P.W. OF SO YEAR O \& $M$ COST (\$)

29. P.W, OF costs 225 \& 28.

(\$)

क) 66,500

5. 18

200

35.4

100

33,7

40. 1

40.10

10.3

95.8

Q2. 0

80. 7

4110

132000

324800

703130

116000

116800
172000

6't200

1668000

1.668600

61200

4.0

1350100

5.09

200

35.4
96

100.

31.5

4. 1

10.2

10.2
94.9

94.9
82.0

82.

$401 \quad 564$

5. 10

3010
3.5 .4

35.14
72

150
29.5

1

50.1

1.1.'

101.1

75.3

5614
400

.79600

55100

$409800 \quad 426400$

1.32000

178900

$322000 \quad 338400$

176400

330600

$70300 \quad 70300 \quad 70300$

$369600 \quad 444600 \quad 263000$

122400

120900

6500

179300

69200

1764900

$180(1)+00$

1800400

1694700

1.7649010

62100 64500

6.5700

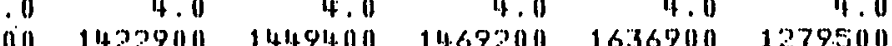

$4.0 \quad 20570$

1311570013586001447400

301890

$3064700 \cdot 3187000 \quad 3249000 \quad 3296800 \quad 3697500 \quad 2852900$

3009110

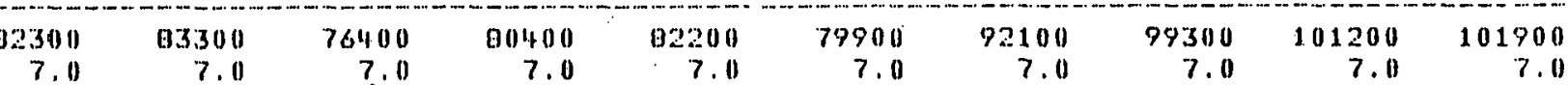

99300
7.0

31. ENERGY CHPI VALUE GRAIETENT (\%)

$32753003315100301005003199700 \quad 3271400 \quad 3179600 \quad 3665400$

32. P.W. OF 50 YEARS OF ENERIGY (\$)

33. P.W. OF RESIIULAL VAL.UEE

(\$)

0

0

402780007.0

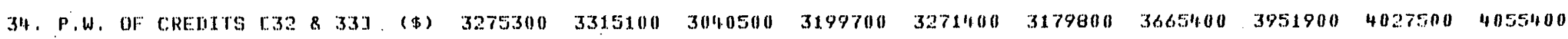

35. P.W: OF NET COST [29-311].(\$) -2561400-250100

147300

50100

25400

507700

812500

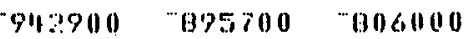

36. RANKBNG WO LEAST MET COST P.W.

61

6.

711.

71

69

75

$? 9$

$2 ?$

23

061100 
NORMANS KILL IYYURO-.ELECTRIC FEASIBILITY STUIY

OPTIMUH CONF IGURATION PROGRAH (OCPHZ)

SO YEAR SERVICE PERIOII PRESENT WORTH ANALYSIS

BASED UPON 50 YEAR EQUIPMENT L.IFE AHI 0.000 PERCENT IIISCOUHT RATE.

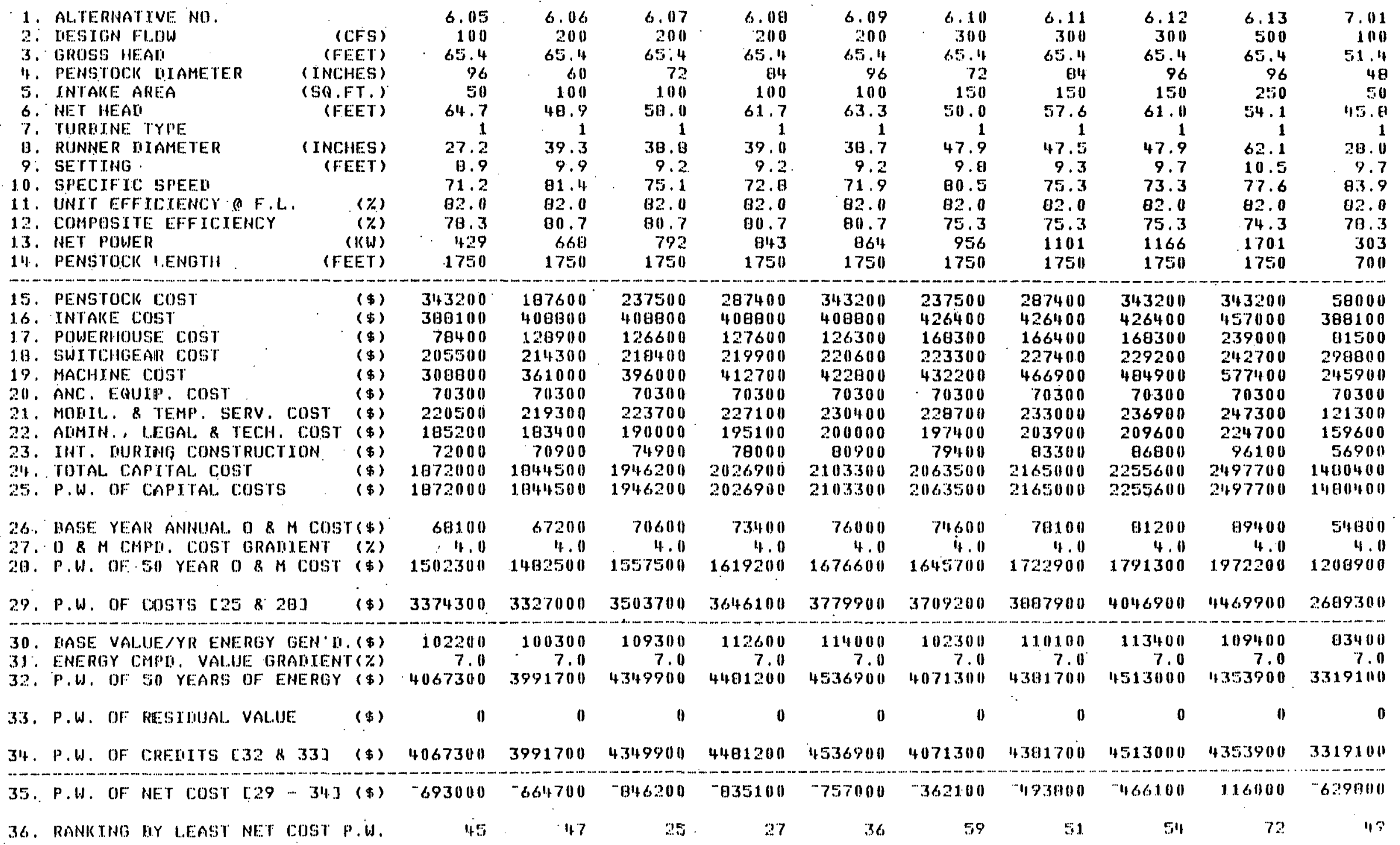


NORMAING KILL HYIURO-ELECTTRIC FEASIBIL.ITY STUIY

OPTIMUM CONFIGURATION PROORAM (OCPHIZ)

SO YEAR SERVICE PERIOI PRESENT WORTH ANALYGIS

BAGED UPIJN SO YEAR EQUIPHENT L.IFE AND B.00O PERTENT DISCOUNT RATE

1. ALTERNATIVE NO.

2. DIEGIBN FLOW

3. GROSS HEAII

4. PENSTOCK MIATETER

5. INTAKE ARE.A

6. NET HEAI!

7. TURTINE TYPE

B. RUNHER IITAMETER

9. SETTING

10. SPECIFIC STEEI

11. UNTT EFFICIENCY O F.L

1.2. COMPOSTTE EFFICIENCY

13. NET POWER

1.4. PENSTOCK LENGTH

1.5. PENSTOCK COST

16. INTAKE COST

1.7. POWERHOUSE COST

1. G. SWITLHGEAR COST

19. HACHJWE COSI

20. ANC. EQUTP. COST

21. MOHTL. \& TEMP. SERV, COST

22. ALMIN. LEGAL \& TECH, COST

23. TOTAL CAPITAL COGS

25. P.W. OF CNPITAL COSTS

$\therefore 7.02$

(FEET) 100

(INCHES)

(SO,FT, )

(FEET)

(INCHES)

(FEET)

$(\%)$

(\%)

(KW) (FEET)

(\$)

(\$) $\quad 82700$

(\$) $\quad 389100$

घ000.0

299800

259100

259100
70300

123200

1625010

58600

1524300

(\$) 1524300

26. RASE YEAR ANIUALL D \& M COST (\$)

27. 0 \& $M$ CHPI. COST GRALIENT (\%)

56.300

4.0

$121+2000$

2766300

29. P.W. OF. COOSTS [25 \& 20]

(\$)

30. HASE VALUE/YR ENERGY GEN IH. (\$)

31. ENERGY CMPI. VAL.UE GRAIIENT $(\%)$

97000

97900

7.10

32.P.W. OF GI YEARS OF ENERLYY (\$)

0

34. P.W. OF CRELTTS C32 \& 333 (\$) 349820

35. P.W. OF NE:T COST [29 -.. 31. J (\$) -731900

41.112

$+6$

37

37

3

33

$\frac{051400}{3812600} 3967800$


NORMANS KILL HYIRO - ELECTRIC FEASJAILITY STUIYY OPTIMUA CONF IGURATION PROGRAM (OCPUIZ)

50 YEAR SERVICE PERIOD PRESENT WORTH ANALYSIS

GASED UPON 50 YEAR EQUIPMENT LIFE ANI G.000 PERCENT IIGCOUNT RATE

1. AL.TERNATTVE NO.

2. TIESIGN FLOOW

3. BROSS HEAI!

4. PENG TOCK LITAMETER

5. INTAKE AREA

b. NET IIEAI

7. TURHINE TYPE

8. RUINNER DIAMETER

9. SETTING

10. SPECIFIC SPEEH

1.1. UNIT EFFICIENCY \& F.L.

12. COMPOSTTE EFFICIENCY

13. NET POWER

14. PENGTOCK LENGTH

15. PENGTOCK TOOST

16. INTAKE COST

17. POUERHIOUSE COST

19. SWITCHISEAR COST

19. MACHIHE COST

20. AHC. EQUJP. COST

21. MOHIL. \& TEMP, SERV, COST

22. AIMIN., LEGAL. \& TECH. COST (\$)

23. INT. IUURING CONSTRUCTION

211. TOTAL. CAIITAL COST

25. P.W. OF CAPITAL COSTS

( $(\mathrm{CFS})$

(FEET)

(INOFT.)

(FEET)

(INCHES)

(FEET)

$(\%)$

(\%)

(KW) (FEET)
7.1207 .13$$
\begin{array}{r}
300 \\
51.4
\end{array}
$$

51.4

96
150

150
+9.3

+9.3
1

47.9

10. 2

日1.1

82.0

75.3

9142

700

$7.13 \quad 0.01$

500
51.4

96

250

$1+6.0$

61. 1

11). 6

63. 7

8.2. 0

74.3

1446 700

6'. 4

40

58.3

3

$24 \cdot 6$

$-3.6$

1.23 .7

B1. 0

Q1.3

401

900

(\$)

(\$) 1451,00

(\$) 426400

(\$) 160300

(\$) 321700

(\$) 439800

(\$) 70300

142800

192300

76300

(\$) 1963300

(\$) 1983300

145400

457000

238000

335300

535500

70.300

153300

207900

65700

$2220+100$

2228400

77000

264400

162000

312600

134300
711300

51000

145100

48700

1265400

1265400
1.265400

8.02 8.03

11) 11
64. 4

60

50

62.1

24.5

$=4.4$

120.3

Q1. 0

日1.3

427

71900 10200

4.7500

4.0

900

$111200 \cdot 136800$

$264400 \quad 264400$

$162000 \quad 162000$

$313700-314100$

$1+1600 \quad 143900$

$\begin{array}{rr}70300 & 70300\end{array}$

$53200 \quad 54600$

$148500 \quad 150000$

$50600 \quad 51900$

$1315500 \quad 1346000$

$1315500 \cdot 1348000$

26. HASE YEAR ANNUAL OI $M \operatorname{COST}(\$)$

27. 0 \& M CMPL. COST GRANIENT (\%)

15361001769200

49200

4.0
500

50300

$4.0 \quad 51400$

$\begin{array}{rr}4.0 & 4.0 \\ 109600 & 1133900\end{array}$

29. P.W. of costs [.25 R 28] (\$) 356910

3997610

2.313300

$2400900 \quad 2458400 \quad 2514100$

30. HASE VALIUE/YR EHERLY GEN I $(\$)$

31. ENERGYY CIPH. VALUE GRADIENT $(\%)$

$101500 \quad 100200$

$\begin{array}{rrrrr}0200 & 100000 & 102900 & 103800 & 104200 \\ 7.0 & 7.0 & 7.0 & 7.0 & 7\end{array}$

4200
7.0

400
7.0

00
.0
00

9800 $113800 \quad 115200$

32. P.W. OF SI YEARS OF ENERISY (\$)

35. P.W. OF NET COST [29-31] (\$) 47010

3997700

0

0

in

0

E1100 45290004584700

3\%. RANKING HY LEAST NET COST P.W.

\begin{abstract}
53
\end{abstract}
$9900-166650$

409520

$\frac{1310004146900415490043698004529000}{45614700}$

o

(5)

\begin{abstract}
67
\end{abstract}

\begin{abstract}
10
\end{abstract}
4.30

(1) 
NORMANS KILL HYIMRO-ELECTRIC FEAGIHILITY GTULY

OPTIMUM CONFIGURATION PROGRAH (OCPUI)

50 . YEAR SERVICE PERIOI PRESENT WORTH ANALYSIS

GASED UPON 50 YEAR EQUTPMENT LIFE AND O.0OO PERCENT IISCOUNT RATE

1. AL.TERHATIVE. NO.

2. MESIISN FLOOW

3. GROSS HEAL

4. PENSTOCK IITAMETER

(CFE)

(FEET)

(INCHES)

(SQ.FT, )

(FEET)

6. NET HEAII

7. TUREINE TYPE

B. RIJNNER DIAMETER

(INCIIES)

(FEET)

9. SPECIFIC SPEED

(FEET)

11. UNIT EFFICIENCY O F.L

12. COMPOSITE EFFICIENCY

13. NET POWER

1.4. PENSTOCK LENGTH

$(\%)$

(KW)

D. PENSTOCK COG

(FEET)

15. PENSTOCK COST

16. INTAKE COST

1. SWITCHGEAR COST

19. MACHINE COST

20. ANC, EOUIP, COST

21. MOHTL, \& TEMP. SERV COST

22. AHMIN, LEGAL \& TECH COST

23. INT. DURINA CONGTRUCT ION

24; TOTAL CAPITAL. COST

25. P.W. OF CAPTTAL COSTS

(\$) 196700

(\$) 196700

276700
162000

329200

2420011

70300

638010

165300

610200

1566201

1566200

26. FASE YEAR ANIUUAL O $\&$ M COST (\$)

27. 0 \& $M$ CMPI. COST GRADIENT (\%)

2月. P.W. OF 50 YEAR 0 \& $M \cos 1$ (\$)

57700

4.0

300

64.4

72

150
57.0

3

43.0

$-3.6$

125.0

81.0

B1.7

1182

$136000 \quad 162500 \quad 196700 \quad 196700$

$162000 \quad 162000 \quad 162000162000$

$337900.340000340900 \cdot 359101$

$295400 \quad 307500 \quad 312700 \quad 115800$

$703000^{\circ} 70300 \quad 70300 \quad 70300$

$64500 \quad 66500 \quad 69500 \quad 75600$

$166300 \quad 169400 \quad 172500 \quad 163200$

$60000 \quad 62600$ 64500 70000

$1581600 \quad 1628600 \quad 1675900 \quad 1041700$

$1581800 \quad 1.62060016759001811700$

29. P.W. OF COSTS $[25 \& 213] \quad$ (\$) $2839100 \quad 2865700 \quad 2947800 \quad 3030400 \quad 3322000$

30. BASE VALUE/YR ENEROY GEN B. (\$) 115300 115400.119100 120600121900

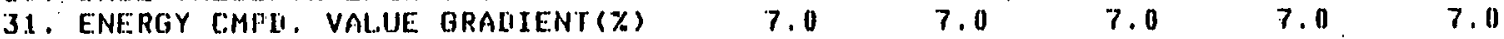

32. P.W. OF 50 YEARS OF ENERGY (\$) $4609600459260047399004799600 \quad 11851300$

33. P.W. OF RESI I UIAL VALUE

(\$)

0

0

ก

0

)

34. P.W. OF CNENITS [32\& 33] (\$) $4600600459260047399004799600 \quad 4951300$

35. P.W. OF NET CoST $[29-34]$ (\$) $-1769500-1726900-1792100-1769200-1529300$

36. RATHKINT HY LEAST NET COST P.W.

7

3

5

13 
ENVIRONMENTAL, SOCIAL, CULTURAL, HISTORICAL

AND ARCHAEOLOGICAL ANALYSIS

The Environmental, Social, Cultural, Historical and Archaeological Analysis has explored and defined the potential impacts of construction and operation of a hydroelectric facility at the Watervliet Reservoir. The environmental analysis performed was categorized into four areas of possible concern: reservoir related impacts, stream related impacts, recreational impacts and other (drinking water) impacts. In addition, a cultural and historical preliminary survey has been performed to document any potentially sensitive areas in this regard. Finally, a determination of pertinent and applicable local, county, state and federal statutes and regulations has been made.

Reservoir Related Considerations

The major impact of any proposed hydroelectric project on the reservoir is related to the lowering of the surface water level. The actual water levels will vary depending on the frequency, . intensity and timing of the proposed drawdown. The maximum proposed drawdown will be approximately three feet, measured from the top of the dam flashboards to the spillway elevation at two-hundred and fifty-nine feet. Once the water level elevation reaches the two-hundred and fifty nine foot mark, all use of the water for hydioelectric generation will cease. The actual timing of these drawdowns will depend upon the eventual marketing scheme, since it will essentially determine the hours of peak demand and thus the times of generator operation.

The reservoir presently fluctuates as much as five feet [Ref. 96] during a given period of time depending on the amount of runoff occurring throughout the watershed or the severity of drought conditions.

The immediate area surrounding the reservoir is predominantly forest land with some wetland areas along the western shore. A 
small golf course, a few residences, highways, croplands and pastures penetrate the reservoir's forest perimeter.

\section{Vegetation}

The western portion of the reservoir contains most of the aquatic vegetation. Plants typically occurring there include cattails (Typha SP), coontails (Ceratophyllum demersum), water lillies (Nymphaea SP) and water chestnut (Trapa natans) [Ref. 97].

A major infestation of water chestnut presently covers sixty to eighty acres in this area. The appearance of water chestnut in a water body has serious ramifications to fishing and useful food and habitat for wildlife. Gradual drawdowns, similar to those presently occurring will permit only a limited number of annual plants [Ref. 94].

The plant itself has very little value to wildlife and tends to crowd out desireable aquatic plants which provide food and shelter to fish and water fowl [Ref. 67].

Operation of the reservoir for hydroelectric generation will have little or no effect on the water chestnut since the seeds of the plant easily drift to areas containing adequate water conditions and remain viable for twelve or more years. The only realistic means of controlling the plant is to physically remove it each year prior to the production of seed pods or by application of chemical herbicides.

The eastern, southern and western shores are forested with trees of the followling species:

American Beech

Ash

Black Cherry

Canadian Hemlock

Locust

Poplar

Red pine

Sugar Maple

White Birch
American Elm

$\mathrm{Birch}$

Black willow

Grey Dogwood

Oak

Red Maple

Redsteili Dogwood

White Pine 
Wildife

The effects of reservoir drawdown on wildife varies especially in relation to the type of wildlife under consideration. It is important to note at this point that there have been no reported sitings of endangered species and no unique habitats identified by the Department of Environmental Conservation [Ref. 100]. The obvious impacts will occur with those species whose Iivelihood is related to the water's edge.

Fish

In June of 1977, a fisheries survey was completed for the Reservoir. In the course of the survey, eleven species of fish were collected or observed. These are:

1. Large Mouth Bass

2. Pickeril

3. Blue Gill

4. Yellow Perch.

5. Black Crappie

6. White Crappie

7. Pumpkin seed

8. White Sucker

9. Walleye

10. Northern Pike

11. Brown Bullhead

The effect of drawdown on fish will again depend on the timing and frequency of the drawdown. The major impact on fish will occur if the drawdown occurs during the spawning and nesting season. Generally, spawning and nesting take place in calm waters that are two to three feet deep. Fish will abandon nests if the water level in their particular area approaches a foot or less [Ref. 97]. If the nests are abandoned, the eggs are open to preditation. If the 
spawning beds are exposed to air, the eggs will die. Typically, this drawdown will have a greater effect on resident game fish such as bass. The major impact on fisheries of a daily drawdown occurring throughout the spawning season would be a major change in the makeup of the fish population and a severe reduction in game fish value. The dominant fish species in waters with a constant drawdown situation are carp and suckers. If the drawdown is gradual or the daily drawdown is controlled during the spawning season, the impact on fish would be greatly minimized.

\section{Biomass Decay}

Concern has been expressed as to the effect of water fluctuations on aquatic vegetation and resulting die-off and decay. The impact of biomass decay on a water body, in general, is reduced water quality and reduced dissolved oxygen levels. The significance of these factors is relative to the quantity of biomass decaying, the time of the year in which die-off and decay occurs and the original water quality. Normally, exposure of fourteen days or more will eliminate most forms of emergent vegetation. However, reservoirs typically have an abrupt blending of terrestrial and aquatic environments. This condition caused by the constant lowering of the water level results in little or no emergent vegetation along shorelines [101].

The lack of impact from decaying biomass caused by the fluctuation of the water level has been suggested by Douglas sheppard of the N.Y.S. Department of Environmental Conservation in his experience with reservoirs in the Delaware Basin. On-site inspections revealed that the exposed shoreline in the eastern portion of the reservoir was virtually free of emergent vegetation.

\section{Effects of Decaying Biomass Eutrophication}

The Watervliet Reservoir is presently considered eutrophic. This term describes a state resulting from the enrichment of waters by nutrients, usually phosphorus and nitrogen. This enrichment occurs naturally in a watershed when water runoff plcks up decaying 
matter and soil particles and deposits them in a water body. The enrichment can also occur as a result of man's activities such as introduction of septic tank effluent or agricultural related waste. The occurrence of these nutrients causes the excessive production of algae and other vegetation. When the plant material dies, oxygen is consumed during decomposition. If a massive amount of organic material is decaying the dissolved oxygen levels of the water can be significantly reduced. If this reduction is severe enough, it will result in problems associated with water quality and $f$ ish habitat. (Most fish require a dissolved oxygen level of between 3-6 mg/ litre of water. Once dissolved oxygen goes below that level, the water is unsuitable for support of fish.)

According to the D.E.C. fisheries survey, approximately $53 \%$ of the reservoir bottom is presently unsuitable for fish production because of low oxygen levels. Chronically low levels will eventually result in an undesireable fish population characterized by carp and suckers. The absence of dissolved oxygen will also lead to the development of anaerobic conditions which pose odor, taste, treatment and filtering problems related to drinking water.

The effects of eutrophication, massive plant growth, can be controlled chemically, however, these chemicals do have serious effects on fish populations as well. A second effect is the reduction of recreational value in cases of severe eutrophication. The present eutrophic condition of the Watervliet Reservoir does not preclude its safe use for municipal or recreational purposes.

Distinctions should be made regarding biomass decay, eutrophiction and water level fluctuations. A eutrophic water body will produce a greater quantity of "biomass" than a similar non-eutrophic water body. This occurs independent of any water level fluctuations. The nature of the fluctuations will, however, determine to a certain degree, what types of plants will be able to grow. 


\section{Other Effects on Vegetation}

The hydroelectric proposal may necessitate some minimal lowering and refilling of the reservoir. This type of drawdown would allow for the establishment of perennial vegetation along exposed shoreline aiding in the reduction of erosion.

The effect of reduced water volume as it relates to crowding of fish populations is not considered serious. Crowding will not adversely affect the $\mathrm{fish}$ if it is temporary' and if water conditions are not unusual. Again it should be noted that the drawdown for hyaroelectric purposes will. cease at the present spillway elevation and will not exaggerate the water level changes presently occurring. Several of the hydroelectric proposals call for modification to the present water intake at the dam. This modification will not cause any localized problems with fish. According to Charles Luckey, operator of the dam, the present intake does not develop enough velocity to draw fish against the protective screen. The only problem encountered to date concerned eels that were able to pass through the protective screen. This is not a common occurrence. The new intake will develop less velocity than its predecessor, thus, there should be no localized problems with fish at the intake.

Waterfowl

There are currently populations of ducks utllizing the Watervliet Reservoir for breeding and brood rearing including mallards, blacks and teal [Ref. 100]. These birds migrate to the reservoir during the spring and fall of the year. Nesting occurs for approximately four to six weeks beginning about May. I.

Lowering the water table daily during the nesting season could impact the breeding population by making the nests, eggs and ducklings more susceptible to preditation by raccoons and other land predators; especially if land links to the nests are exposed. [Ref. 90]. Conceivably, certain vegetative food types for the ducks could be disrupted. Conversely, establishment of permanent emergent vegetation would provide an additional food and habitat resource. 
It is questionable whether the hydroelectric option would aggravate present conditions since the water level in the reservoir presently fluctuates.

Other Wildife

With the exception of a fisheries survey, no wildlife inventory has been conducted in this area. If a specific development proposal is forwarded. for this area, the N.Y.S. Department of Environmental Conservation will conduct one.

Certain species of wildlife are known to inhabit this area. Resident wildlife is representative of species occurring in a sociation with the water's edge and with typical local habitats such as muskrat, raccoon, fox, white-tailed deer and a variety of commonly occurring reptiles and amphibians.

Perhaps the most obvious wildlife inhabitant is the muskrat. On-site observation has verified that a number of the animals currently occupy the western portion of the reservoir. The effect of the proposal is related to the fact that muskrat utilize water as a protective barrier against predators. Drastic drawdowns could expose their huts to land predators. It should be noted, however, that the muskrat population has successfully adapted to the fluctuations presently taking place. The effect of drawdown on their main food source; cattails, appears to be minimal as evidenced by a large cattail community in the western portion of the reservoir (Figure 45).

Erosion

The effects of rapid drawdown on reservoir embankment stability is more a function of erosive forces rather than moisture content. According to the Department of Agriculture and Soil Conservation Service, wind and ice action on the toe of embankments presently accounts for most of the shoreline erosion problems.

Tree growth on affected shorelines seems to have little impact on preventing erosion. There seem to be two possible ways of reducing the erosion potential of shorelines. One is to place a 
gravel blanket on affected areas as per N.Y.S.D.O.T. specification. The other is to establish specialized emergent vegetation to reduce the effect of wave action. These perennial plants would be most successful in an environment where there is a daily fluctuation of the water table rather than constant slow lowering [Ref: 94].

The raising and lowering of the reservoir will also slightly effect water table levels in the immediate area. The conditions forwarded by this proposal are not unlike those presently occurring and will thus have little increased impact.

\section{Siltation Disturbance}

An additional reservoir-related concern is the impact of the overall hydro proposal on siltation characteristics. Siltation is occurring at all times and occurs mainly as a result of erosion throughout the watershed. When silt particles reach the reservoir, they are deposited in areas of reduced water velocity and depth. The major siltation deposits occur where the Bozen Kill and the Normans Kill enter the reservoir.

In addition, siltation occurs from the erosion of surrounding banks and shoreline. The major cause of bank erosion in the Watervilet Reservoir is an unstable soil condition aggravated by water and ice action on the toe of steep slopes. Affected areas are illustrated on the surface conditions map.

Generally, increased siltation or disturbance of silt deposits affects a water body in several ways. The turbidity level of the water increases posing immediate concerns in terms of water quality. Many sediments are also nutrients and can thus further promote eutrophication. Siltation affects the useful life span of a reservoir by constant infilling of the lake bottom. In addition, transported sediment may also affect the operation of water-related machinery, although this is not presently a problem at the reservoir.

The effect of the proposed hydroelectric facility on siltation wl1l not be significantly different from present levels since the limits of water fluctuation will remain essentially the same. The 
added freguency of arawdown will not increase erosion levels. As previously stated, bank erosion is aggravated by wave and ice action at the toe of steep slopes. Erosion would be more severe if the water level were raised above present levels. Daily drawdown of the water level coupled with a bank stabilization program will actually reduce local erosion potential.

An additional reservoir-related impact will occur due to modifications to the dam. The hydroelectric project will necessitate construction of a large superstructure on the reservoir side of the dam. This superstructure might possibly be constructed on land prior to coupling with the dam. A construction site near the dam will have to be established and an assessment performed analyzing specific impacts.

The types of impacts expected relate to the clearing and grading of the site, erosion from exposed soil, impact from construction machinery and disturbance of the shoreline in the immediate construction area.

These impacts will be temporary and should constitute no irreversible environmental impact. To the extent possible, these effects should be mitigated by responsible construction techniques.

\section{Stream Related Considerations}

\section{General}

The impact area. under consideration is directly downstream from the dam and consists of the Normans Kill Creek bed and immediate environs, including a two-hundred and fifty foot strip on the northerly creek bank from the dam to the existing powerhouse facility. The creek bed itself is exposed shale. The water flow over this bed is variable. For most of the year, it receives a steady flow of water from the reservoir. For temporary periods, however, usually in the summer, there is virtually no flow between the dam and the powerhouse. This condition lasts for periods of up to three months, although the average dry period is shorter [Ref. 96]. This occurrence of a no-flow condition severely limits the aquatic environment in this portion of the creek. 
The surrounding land is mostly forested although there is some farmland abuting the immediate impact area. Just downstream from the existing powerhouse facility is a wetland (as indicated on the vegetation map) that has been identified by N.Y.S.D.E.C. as significant and will be a consideration in assessing the possible impacts of the project.

slopes in the area vary although the existing powerhouse rests on relatively flat land. The area adjacent to the creek near the dam is quite steep with slopes ranging from eight to fifteen percent or more. It should be noted that a bedrock outcropping occurs on the north stream bank within the project area [Ref. 103]. Soils are generally favorable to development activities. In addition, there are two roads in the area, French's Mill Road and Fuller station Road. ConRail maintains two railroad trestles across the Normans Kill in the immediate project area.

The hydroelectric proposal considers eight development options for the eventual generation of power. These options will impact the immediate environment in several different ways depending mostly upon their physical location.

The basic areas of impact consideration are the two-hundred and fifty foot strip previously decscribed, eight-hundred foot strip of the stream bed and the area of the existing powerhouse facility.

\section{Vegetation}

Terrestrial vegetation in the form of indigenous trees and understory will be removed during the construction process if the options one, two, six and seven are followed. The removal is necessary in order to provide access to construction machinery and to allow placement of the Penstock.

Erosion and sedimentation

For all options involving installation of the new Penstock, excavation will take place either for burial of the pipe or construction of footings. Where this excavation takes place on the stream banks (Options $2,5,6,7$ ), there is a high potential for soil 
erosion which is further compounded by the nature of the slopes. Excavation in the stream bed will have a significantly lower potential for erosion since the slope is much less and the component material is bedrock. Sediment and rubble, however, could be introduced into downstream and wetland environments.

The soils in the upper excavation areas and in the existing powerhouse vicinity are not highly erosive by nature. As all options approach the existing powerhouse, the slopes reduce significantly as does the resulting erosion potential.

In conjunction with the various penstock options, several powerhouse generating and switching facility options are under consideration. The potential environmental impacts are essentially the same.

With the exception of Option 8 , construction and facility operations will impact the historic resources of the area. The extent of possible disruption can be determined when the historic assessment is completed.

None of the construction options present irreversible, detrimental environmental impacts. Mitigating measures are expected to be undertaken to prevent undue environmental stress during construction and operation of the facility. Areas disturbed by excavation activities will be stabilized by backfilling and replanting to prevent any long-term erosion potential.

Construction activities for this portion of the project will take place during the no-flow period of the stream. Excavation material will have to be removed from the site and deposited in an approved spoil site; thus being prevented from entering downstream aquatic and wetland environments.

In the event that the stream does not dry up due to an especially wet year, special measures will have to be taken to divert water around construction activities.

The option with the least overall impact on the environment is option no. 8 which involves the burial of the Penstock and generating facilities in the stream bed. Erosion and sedimentation potential are diminished as is any potential visual impact since the stream bed will be rehabilitated to its preconstruction condition. 
Wildife

Wildife resources in the immediate construction areas are not expected to be severely impacted. The land area in question is not considered a significant wildlife habitat. The aquatic environment is presently severely limited as to the variety and quantity of fish and plant life due to the fact that the stream bed. is in a no-flow, no-water condition for portions of the year. The most probable, potential impacts to wildife will occur downstream from the project where water flows are more consistent.

The fish population in the viable portions of the Normans Kill was surveyed in June and september of 1972 by N.Y.S.D.E.C. At that time, the following species were found:

$\begin{array}{ll}\text { Striped Bass } & \text { Cutlips Minnow } \\ \text { American Shad } & \text { Blacknose Dace } \\ \text { White Perch } & \text { Common Shiner } \\ \text { Pumpkinseed } & \text { Johnny Darter } \\ \text { American Eel } & \text { Largemouth Bass } \\ \text { White Sucker } & \text { Brown Bullhead } \\ \text { Carp } & \text { Rock Bass } \\ \text { Goldfish } & \text { Creek Chub } \\ \text { Spottail Shiner } & \text { Bluegill } \\ \text { Golden Shiner } & \text { Northern Hog Sucker } \\ \text { Smallmouth Bass } & \text { Longnose Dace } \\ \text { Redbreast Sunfish } & \text { Banded Killifish. } \\ \text { Falfish } & \text { Redfin Pickerel }\end{array}$

Facility Operation

Operation of the facility itself will slightly reduce the average stream release during normal periods and slightly lengthen the time of no-flow condition between the dam and powerhouse. These factors are not expected to be of such a magnitude that they will further aggravate the impacts presently occurring. 
Each of the options will release reservoir water into the Normans kill at a powerhouse site. This release will have several downstream benefits. The most significant is the re-oxygenization of the water entering the stream.

Powerhouse locations (Options 2, 7 and 8) are just upstream from the Town of Guilderland sewer treatment outfali. The re-oxygenated releases at this point will aid in improving the ability of the stream to process this material. The rehabilitation option will release below the sewer outfall and will have a somewhat lesser although positive impact on water quality.

\section{$\underline{\text { Recreational }}$}

Recreational Impact

The City of Watervliet and the New York State Department of Environmental Conservation have entered into a cooperative agreement that will allow public access and fishing, via a permit process, to portions of the Watervliet Reservoir. The fishing activities will necessitate the construction of several parking lots and access trails to designated fishing spots (Figure 46).

The drawdown of water, if conducted in a manner that responds to the spawning cycle of game fish, will not have a significant impact on fish or fish production within the reservoir. As mentioned earlier, temporary crowding of fish does not constitute a major detriment to aquatic life. Drawdown could affect access to the water in areas of gradual shoreline drop-off

The most. significant impact on fish populations is the low dissolved oxygen content in the water. The eutrophic condition of the water does not permit the stocking of salmonoides (trout), however, a significant number of bass and pan fish will continue to make the regervoir a popular fishing spot.

As part of the recreation agreement, ice-fishing will be allowed. Drawdown of the reservoir as prescribed by hydroelectric operations will not affect overall ice safety. Once an ice layer forms on the lake, it wlll naturally bend to meet lower water levels 
without becoming unsafe. The only effect on the ice would be at the edge where a certain amount of cracking could occur.

The hydroelectric proposal, as previously discussed, will release water back into the Normans Kill at the powerhouse site. This water will be re-oxygenated, to a certain extent, at its point of release. This water will have a beneficial impact in the immediate area of release by increasing the water's ability to support aquatic life and to process waste materials.

\section{Other Impacts}

There are no other impacts on recreation as a result of the hydroelectric generating facility. A combination of factors, however, do place constraints on certain uses. The fact that the reservoir is a drinking water supply coupled with the relatively small amount of land held by the city around the reservoir would limit the types of activities that could otherwise take place. In addition, there has been a lack of cooperation between some of the surrounding landholders and the Department of Environmental Conservation concerning easements for recreational purposes.

Drinking Water Related

The Watervilet Reservoir is presently considered a eutrophic water body. As such, it is subject to algal bloom, low dissolved oxygen levels and resulting anaerobic growth which all affect water quality to a certain degree. Generally speaking, eutrophication does not make water unuseable for municipal purposes; it only necessitates the use of special filters and chemical treatment. The vegetation in the reservoir can and has been treated with copper sulfate to reduce the impacts of eutrophication. The chemical is not known to have an effect on humans, however, it does collect in sediment layers and effects phytoplankton and algae. These factors impact fish populations by reducing food sources. In some cases, eutrophic water has taste and odor problems, however, this is not yet a problem at the Watervliet Reservoir. 
Concern has been expressed regarding suspended solids and turbidity and the effect the proposal will have on increasing these levels. These factors do have an effect on water quality. The immediate concern to the Watervliet Reservoir is somewhat questionable, however, presently the western end of the reservoir with its wetlands and shallows is acting as a natural filter for the water supply. Water quality samples from representative portions of the Reservoir illustrate that the municipal intake is at the eastern end. For this reason and the fact that the hydro proposal will not significantly alter turbidity and suspended solid levels, these factors are not expected to adversely affect the water supply.

\section{Cultural and Historical}

Prehistoric Cultural Resources

Archeological evidence indicates that Albany County was an area of considerable prehistoric activity, but not one of extensive prehistoric settlement [Ref. 25]. Native American populations transected the region with trails running between the excellent riverine resources of the Hudson and Mohawk. Trails have been found along the banks and high bluffs of these major rivers; as well as along their tributaries. [Ref. 93]. Numerous sites have been recorded along the Normanskill in close proximity to the project area [Ref. 25]. The Nott site [Ref. 76] and the Lizzie I and II sites have recently been reported along the Hunger Kill near Route 20 in Guilderland [Ref. 65]. These three sites date to the Archaic Period and diagnositic artifacts indicate sporadic occupation from 3500 - 2500 B.C. These camp/workshops were probably used during the spring-summer phase of the seasonal cycle, when substantial numbers of people would gather at low-lying streams and creeks to exploit fish and aquatic food resources. With the advent of cold weather, freezing of streams and southward migration of birds, these groups were forced to split into smaller nuclear units and move inland to back-country camps, where they exploited deer and other browsing animals. Route 20 follows a former. Indian trail, and sites have 
been reported along its path at Dunnsville, Fullers station and Mcknowville [Ref. 25]. Evidence of Indian settlement from all three phases of prehistoric occupation (Paleo-Indian 10,500-8,000 B.C.; Archaic 8,00001,000 B.C.; and woodland 1000-1500 A.D.) are found in the Pine Bush region [Ref. 26]. During the early historic period, Albany County was inhabited mainly by the Manikan Indians, with the Schaghticoke occupying scattered, small settlements [Ref. 25]. In 1730, about the time of Europena settlement, several Indian villages and "work places" existed in the Guilderland area [Ref. 66]. Within the proposed project area a campsite is recorded at French's Mills, near the falls [Ref. 25]. T. Cassavant records "the largest and most productive of Indian sites around Albany" is the Vosburg Farm site which is situated on the north bank of the Normans Kill, about one-half mile from French's Mills. The site lies on a flat hill that slopes down to the creek and has produced pestles, gouges, chisels, celts, bannerstones, steatite pottery, semilunar choppers, sinewstone knives, drills, spears and projectiles. Many scattered artifacts have been found directly opposite the Vosburg site, extending along the south side of the creek and on the flats near the mills. "Camp sites seem to be abundant in this vincinity" [Ref. 25].

This report is confirmed by the residents of the area who report finding artifacts when farming and gardening in the area surrounding the reservoir. This conforms to the model of prehistoric settlement. patterns that predicts a higher density of site locations along the Hudson floodplain and inland streams [Ref. $69: 6]$.

\section{Historical and Cultural Resources}

Permanent settlement of the Albany area began in 1629 when the Dutch West India Company initiated a policy of rapid settlement of New Netherlands in order to increase the power and wealth of the Company. Kilian Van Rensselaer, a pearl and diamond merchant in Amsterdam and a director of the Dutch West India Company, took advantage of the Company's policy. In 1630 he purchased his first 
tract of land from the Indians, and within seven years, his "manor" extended for twenty-four miles on each side of the Hudson River, embracing what is now Albany, Rensselaer and part of Columbia Counties. It was the duty of the Patroon to encourage settlement of his land and in the years following 1630, colonies from Holland came to the region and were provided with homes and land leases in Fort orange (now Albany) and the surrounding area. Albany County, one of the original ten counties of New York state was formed in 1683 . Modifications of the county's boundaries continued throughout the 18 th and 19 th centuries, until its present limits were firmly established in 1809. The Town of Watervliet, formed on March 3 , 1788, originally encompassed the Town of Guilderland. When the two towns were separated in 1803, Guilderland included Knowersville (now Altamont), Guilderland Center, French's Holiow and Hamiltonville (now Guilderland). The town was well settled prior to the Revolution. A 1767 map of the Manor of Rensselaerwyck (Figure 49) indicates there were 148 families living west of the Hudson River. A battle of the Revolution, the Battle of the Normans Kill was fought in the town during August 1777:

"On the 13th of August (1777) the same day on which col. Harper so opportunely led troops to Schoharie, Lt. Col. Schermerhorn proceeded to the Normanskill with a body of Schenectada militia, and forty Rhode Island troops, - in all about 100 men - to root up a rory gathering at that place. The expedition was very successful. David springer, a noted Royalist was killed. Thirteen of his comrades captured, the remainder dispersed and confidence again restored, where all was doubt and disaffection, without the loss of a single man on the part of the Americans." [Ref. 12]

The town continued to expand both socially and economically after the Revolution. One of the early industries of Albany County, a glass factory, was established in Hamiltonvilie (now Guilderland) in 1792. The company manufactured window glass and by 1813 
according to Spaffords Gazetteer, 500,000 feet of window glass were produced annually [Ref. 25]. The procedure for making glass required large quantities of wood. Much of the land in the vicinity of the factory had been cleared and the cost of transporting wood from increasing distances eventually made the glass business unprofitable and it closed in 1815. In addition to the glass factory, the other early industries in the town were the cloth factories at Sloans (now Guilderland), Knowersville(now Altamont) and French's Mills and several flour and grist mills [Ref. 25]. There were few saw mills due to the scarcity of lumber [Ref. 16]. Most of the people were engaged in farming.

As additional roads were laid out and improved, postal routes and stage lines were established and numerous taverns were opened along the roadside. One such tavern was established at French's Mills in 1800 by Jacob Aker. The railroads, especially the Athens-Schenectady (West Shore) line, gave impetus to the economic growth of the community. By 1896, the Town of Guilderland had a cider and vinegar factory, a sash and door factory, a general store, a bootery and shoe factory and two hotels [Ref. 25].

Within the project area, the 1767 Bleecker Map (Figure 49), depicts the homestead of Col. Abraham Wemple. The Wemple house was built in 1760 by Vrooman, who used the clay deposits near the barn to make the bricks for the house. Over the course of the next two and one-half centuries the house was owned by the Wemples, sigsbees, Myers and Woodrichs. It was destroyed when the Reservoir was flooded in 1915 [Ref. 12]. In 1795, Peter B. Broeck established "clothing works" at French's Mills. Abel French established another clothing factory there in 1800 [Ref. 16]. A grist mill was also located on the creek at French's Mills [Ref. 66, 92]; Gregg, personal communication, 1978). By the 1880's, the mill properties had passed from their original owners to the Reynolds and from the Reynolds E. Spawn \& Co. Spawn was the proprietor of the flour and feed mills and grown to a population of 450 [Ref. 16]. By 1896, the mills were no longer operating, although the buildings associated with its operation remained standing [Ref. 25]. 
The house (Figures 53, 54, 55), located on the northeast side of the bridge was formerly owned by the E. Spawn family. The house was probably built about 1820 . It has a double stone foudation and the original wide planked wood floors. The oldest wing of the house is centered around the kitchen, whose interior doors contain the original hand-wrought iron latches. The main entrance has etched sidelight and transom windows. The house is currently owned by Mr. and Mrs. Pospisil.

Prior to the flooding of the Reservior "when the Normanskill and Bozenkill moved sluggishly to their confluence... the meadows that bordered the banks of the stream were held to be the richest farm lands in the Town of Guilderland." [Ref. 12]. The Reservoir was flooded in 1915. The covered bridge across the creek was replaced by an iron structure in the early 1930's [Ref. 98] (Figure 52).

Analysis and Recommendations

The literature search, research and interviews indicate there is a high potential for finding evidence of both prehistoric and historic activity directly within the proposed project area. The extremely sensitive nature of the area surrounding the reservoir mandates that further archeological investigation and field reconnaissance be undertaken. Snow cover made it impossible to conduct a general walkover of the area. This will have to be done in order to resolve conflicting reports [Ref. 92] as to the existence of foundations associated with the early mills on the creek. Subsurface testing must also be done to determine if the proposed construction or its associated activity (this would include grading, movement and/or storage of heavy equipment, construction of temporary access roads or field headquarters, etc.) will disturb any buried prehistoric or historic site. If testing will have to be done to delineate its boundaries. If the site cannot be avoided by the proposed construction activity, then a survey must be done in order to obtain and adequate sample from the site and precautions must be taken (i.e. fences crccted) so that minimal damage is inflicted upon the site. 


\section{Pertinent Statutes and Regulations}

National Environmental Policy Act of 1969 (N.E.P.A.)

The act was established to underline the federal government's responsibility to reduce adverse environmental impacts and to assure, where possible, the long term enhancement of the environment resulting from federal programs and projects. The act requires that proponent federal agencies assess projects and policies in terms of their environmental impact, adverse impacts (if any), alternatives to the proposed actions, long-term social effects and any irreversible impacts that might occur as a result of the proposal [Section 102 (2) (C)].

The Basic procedure requires that the environmental assessment be submitted to the Environmental Protection Agency by the recipient of the federal funds. This assessment will be utilized to determine if an environmental impact statement need be prepared and to prepare one if required [Section 104 (b) (c)]. The act prohibits any final administrative action on the proposal until the environmental review and determination is complete [Section 108 (A)].

Environmental Conservation Law 8-0101 ART 7 - (S.E.Q.R.)

The purpose of S.E.Q.R. [Section 617.1 (C)] is to promote efforts which will prevent or eliminate damage to the environment from various programs, projects and actions undertaken by a state or local agency and which balance ecological factors with social and economic factors [Section 617.1 (D)].

In the broadest sense, the act allows for environmental review, by the appropriate state or local agency of any public project undertaken or funded by that agency or any private project requiring a permit from a state or local agency [Section 617].

The initial action by the reviewing agency under S.E.Q.R. is to determine the significance of a proposed action. 'If it is determined that the project will have little or no impact, it is considered. a Type II action and no further review is required. If, 
however, the lead agency determines that the proposed action will have a significant impact, an environmental impact statement will be required [Section $617.4(\mathrm{~A}-\mathrm{J})$ ].

The lead agency may, in light of its review, approve or reject funding of the proposed project or approve or reject granting of the various permits needed to initiate the project [section 617.5].

When the proposed project involves a federal agency and a draft and final environmental impact statement is prepared under the National Environmental Policy Review. Act of 1969, that agency will: have no obligation to prepare an additional EIS for the state. However, the project will still be subject to compliance with S.E.Q.R. and final federal actions are not controlling on any state or local agency under this act [Section 617.16].

Freshwater Wetlands Act - N.Y.E.C.L. Sections 24,0101 New York State Regulation - 6 NYCRR 662 - Wetlands Filling

New York State, recognizing the value of freshwater wetlands has made it a state policy to preserve, protect and conserve freshwater wetlands, to prevent despoilation and destruction and to regulate the use and development of these wetlands. As part of this act, the state has, or is in the process of defining and mapping significant wetlands within the state. In general, a freshwater wetland is considered significant if it has an area of at least 12$4 / 10$ acres, or if less than $12-4 / 10$ acres, is of unusual local importance as determiner hy the Commissioner of Environmental Conservation.

Any person desiring to conduct, on freshwater wetlands, as designated, any form of drainage, dredging, excavation, dumping, filling, construction of structures in or any activity which substantially impairs the functioning of a particular wetland will require a permit from the appropriate local governing body or the commissioner.

Certain activities related to agriculture and public health activities are excluded from regulated activities. 
The New York State rules and regulations are promulgated under Freshwater Wetlands Act concerning interim wetlands permits. These regulations are presently being updated to comply with S.E.Q.R. The new rules and regulations are expected by May, 1979.

New York State Environmental Conservation Law Article 15 water Resources - New York State Regulations 6 NYCRR 608 - Use and Protection of Waters

New York State Environmental Conservation Law, Article 15 gives statutory authority for the part 608 regulations. Section 608.1 of the regulations states that no person or public corporation, other than a state department or a state public corporation shall change, modify or disturb the course or bed of any stream classified as $A A, A A(G), A, A(T), B, B(T)$, or $C(T)$ without having applied for and obtained a written permit from the Department of Environmental Conservation. No permit will be required for local public corporations if the corporation has entered into a written memorandum of understanding with the Department of Environmental Conservation outlining the procedures to be followed in completing the activities affecting the system.

In addition to stream bed and bank modifications, a permit is required for any construction, reconstruction or repair of any dam unless a permit has been obtained. All applications concerning dams are forwarded by the local permit agent to the central permit agent to the Albany office. Generally, the application for permit concerning a dam will also be forwarded to the state Department of Transportation. These regulations will be revised by February, 1979 to comply with S.E.Q.R.' requirements.

Fish and Wildlife Coordination Act 661-666

section 661 recognlizes the vital contribution of wildife resources to the nation in light of public interest and significance due to expansion of national economy. This section provides means in which wildlife conservation shall receive equal consideration and be coordinated with other features of water resources development 
projects. Authority to administer this act is given to the Secretary of the Interior. The secretary is authorized to (1) provide assistance to and cooperate with federal, state and public or private agencies in development and protection of wildife resources and their habitat, (2) to make wildlife surveys and investigations of the public domain, and (3) to accept donations of land and/or funds to carry out the purpose of this act [Section 661]. Whenever the waters of any stream or other body of water are proposed or authorized to be impounded, developed, the channel deepened or the stream or other body of water otherwise controlled or modified by a federal agency or by any agency under federal permit or license that agency will consult with the U.S Fish and Wildlife Service, Department of Interior and with the head of the state agency with administration over wildlife. This consultation will discuss the impacts of the project development on wildlife. The reports and recommendations generated by this consultation shall be responsible for authorizing or licensing the project [Section $662]$.

Any project covered by this act will make adequate provision, consistent with the primary purpose of the project for the use of these waters and adjust lands for conservation, maintenance and management of wildlife resources and habitat [Section 663].

The act also covers the administration of lands made available to the Department of Interior for wildlife conservation purposes and provides for sonperation between the secretary and the state department responsible [Section 664].

Endangered Species Act of 1973 - 16 USCA SS 1531-1543

Provides for the protection of threatened or endangered species as identified in the rules promulgated under the act and gives the secretary of the Interior authority to issue prohibitions deemed necessary under section 9 (A) (I) of the act relating to any endangered species. 
New York State Agricultural and Districting Act (New York Agriculture and Markets Law, Article 25AA)

The intent of the agricultural districting legislation is to encourage the continuance of a strong agricultural industry by allowing protection from a portion of the expanding costs of public facilities and services and to provide adequate hinderances to residential, industrial and commercial development within established agricultural districts. Through county legislation, farmers may form agricultural districts which give them special considerations under New York state Department of Environmental Conservation law.

In short, local ordinances cannot restrict structures and activities normal to farming: public agencies cannot take farmland in an established district without special justification; special public service districts for sewer, water, lights or non-farm drainage may not impose special ad valorem levies on land used for agricultural production beyond a house and lot within an agricultural district; property tax assessment may be made an agricultural instead of market value.

There are no agricultural districts within the project area.

Open Space Land Act - 42 USCA Sections 1500 - 15,000

Provided. for a federal program to curb urban blight to encourage more economic and desirable urban development, to assist in preserving areas of historic, recreation and conservation value by making grants for land purchase.

Authority to make grants was terminated after January 1, 1975

Migratory Bird Act - 16 USCA Sections 715-7155

This act established a federal commission, headed by the chairman of the Interior Department to consider and pass upon any area of land recommended for purchase, rental or as a gift for migratory bird refuge.

It also gives the Department of Interior authority to control activities in designated national wllalife refuges. 
Local Zoning - Local Law No. 1 of 1971 - Town of Guilderland The Town of Guilderland has a zoning ordinance in effect, promulgated under its authority to protect the health and welfare of its citizens.

The zoning designations in areas adjacent to the reservoir are agricultural, industrial and light commercial.

Under normal circumstances, the zoning board reviews projects as to conformance with zoning regulations and either.grants or denies permits for conflicting uses.

Hudson River Valley Commission - New York Executive Law 721

The law provides for review of any project occurring within one mile of the Hudson River or two miles if visible from the River. The purpose of the law was to protect the scenic, recreational and natural resources of the Hudson River Valley. The Agency has advisory power only and cannot veto a particular project. The commission does, however, have injunctive power in order to force submission of projects for review.

The Commission has not fully funded recently and has not reviewed any projects since 1976. In addition, all of the Commissioner's terms have expired.

Freshwater Wetlands Act - N.Y. E.C.L. and N.Y.S. Regulations 6 NYCRR 672

The regulations are promulgated under the wetlands act. and refer to any wetlands affected by the project. Significant wetlands do occur in the immediate project area and adjacent to it. A wetlands permit will likely be required since there will be a possible impact on these wetlands.

Fish and Wildlife Coordination Act

If N.E.P.A. review regulations are in effect, the project will be reviewed by various federal agencies. "This act insures that the project be reviewed by the secretary of the Interior and his 
comments noted concerning $f$ ish and wildlife impacts in any final determination.

The act would also apply if any federal permits, such as an Army Corps of Engineers permit, were required.

\section{Local Zoning Law}

The local zoning board, in actions involving the state or federal government cannot actually deny construction of the project. However, the impact of the project in light of local zoning regulations will be noted under S.E.Q.R. review by the Guilderland zoning Board.

\section{Statute Applicablitity}

The previous description of laws and acts was comprehensive in nature, forwarding a basic description of potentially applicable laws but not determining whether the given law actually affected the reservoir project. The laws basically define the roles of the federal, state and local governments. in environmental and land-use decision making. The various laws are forwarded to insure that projects conform to the public interest. The following laws and acts apply specifically to the proposed project.

The National Environmental Policy Act of 1969 and the New York State Environmental Quality Review Act

These are federal and state laws with similar goals, the applicability of. which depends on the funding agency involved. If it is determined that the project is a federal project, the applicant will go through the N.E.P.A. assessment process and will most likely necessitate the filing of an environmental impact statement with the sponsoring federal agency. If an impact statement is required under N.E.P.A., it will satisfy similar requirements under S.E.Q.R. The review of the project, however, will go through federal, state and local channels, especially since various state permits will be required. 
If it is determined that the project is state funded, it must conform to S.E.Q.R. regulations. The S.E.Q.R. process must be instituted and a determination made as to the type of impact resulting from the project prior to the issuing of any other state or local permits.

The sources contacted by the consultant at the New York state Department of Environmental Conservation and the New York state Energy Research and Development Authority indicated that the project will most likely fall under S.E.Q.R. review. In any case, the project will necessarily be subjected to an environmental impact review.

New York State Regulations 6 NYCRR 608 are the regulations promulgated under Article 15 of the Environmental Conservation Law. The project will be subject to stream protection permits and review by the Department of Environmental Conservation.

General Assessment - Historic Preservation Laws

Historic Sites; Buildings and Antiquities Act 16 USCA SS461-467

Section 461 of the Historic Sites, Buildings and Antiquities Act establishes as a national policy the preservation and protection of significant historic sites, buildings and objects for the benefit of the American public. The power to administer this national policy is given to the secretary of the Interior ("Secretary") through the National Park Service. The Secretary's responsibilities, enumerated in section 462 , lnclude conducting surveys to identify historic and archeological sites, acquiring title to such sites, insuring the preservation of such sites; establishing museums, erecting site markers and providing pertinent site data to the public. The secretary also has the power to appoint an eleven member advisory board [Section 463] and to use congressionally appropriated money to pay for required technical and professional services [Section 464]. Congress is given the authority to allocate the funds necessary to carry out the stated 
policy [Section 466]. The laws enumerated in this title take priority over any other laws relating to similar subject matter [Section 467].

National Historic Preservation Act of 196616 USCA SS470 et. seq. The primary thrust of the National Historic Preservation Act of 1966 is the expansion and encouragement of historic preservation programs and activities by the Federal government. The secretary of the Interior ("Secretary") is authorized to maintain and enlarge a national register of districts, sites, buildings, structures and objects significant in American history and prehistory. The Secretary is further authorized to establish a matching grant-in-aid to states for projects that preserve historic sites and to establish a matching grant-in-aid to the National Trust for Historic Preservation in the United States. [Section 470a]. Grant applications must be submitted to the secretary in accordance with both his prescribed rules and with the established state-wide historic preservation plan. The grantee is responsible for procuring from 30\%-50\% of project funds from sources outside of the Federal government and for assuming upon completion of the grant, maintenance and preservation costs [Section 470b]. The Department of Housing and Urban Development is authorized to make grants to aid in the restoration and preservation of buildings of architectural and historic significance [Section 470b-1]. The secretary has the power to apportion funds for state-wide surveys in accordance with priorities established by him [Section 470c]. Federal assistance is available from only one Federal agency per project [Section 470d].

prior to the approval of funds or the granting of a license for any Federal or Federally assisted project, the effect such project may have on a site that is on, or is eligible for the National Register of Historic Places must be considered and submitted to the Historic Preservation Advisory. Council for comment [Section 470f]. This Advisory Council is an in dependent agency of the United States whose purpose it is to render advice and make recommendations relative to the national policy for historic preservation [Section 470i]. 
Archeological Conservation ACt of 1974 - 16 USCA S469

Sections 469-469c provide for the preservation and protection of archeological and historic sites that may be destroyed or irreparably damaged by flooding, road construction, erection of workman's communities, relocation of railroads, highways and other alterations to the land caused by the construction of dams, or any Federal or federally licensed activity or program.

Pursuant to Section 469a, the Secretary of the Interior ("Secretary") must be given written notice before a license can be issued for dam construction. This notice must indicate both the location of the proposed dam and the approximate area to be flooded or altered by the planned construction. However, if (1) a floodwater retarding dam is to be built which provides less than 5,000 acre-feet of detention capacity or (2) any type of dam creates a reservoir of less than 40 acres, then the provisions of this section apply only if the construction company finds during preliminary surveys that historic or archeological sites exist or may be present in the proposed reservoir area.

Section $469 a-1$ provides that if a Federal agency finds or is notified in writing that a federal construction project or a federally licensed project, activity or program may cause irreparable loss or destruction to a significant historic or archeological site, the agency must so notify the secretary in writing. After providing the secretary with information concerning the nature of the project, the agency may request the secretary undertake the recovery, protection and preservation of data from the site, or the agency may undertake the activity itself. Copies of any reports prepared pursuant to this section should be forwarded to the secretary.

When a Federal agency provides financial assistance to a private or public concern, the secretary may conduct a survey of the affected area and recover data with funds appropriated expressly for this purpose. The secretary may provide compensation for damages that result in delays in construction or in temporary loss of use of nonfederally owned lands. 
If upon notification, the secretary determines that significant historic or archeological data is being destroyed or irrevocably lost by any federal or federally licensed project, he may conduct or cause to be conducted a project to recover and preserve such data. No survey or recovery work can be required if it will interfere with projects undertaken in recognition or anticipation of an emergency or national disaster. The secretary must initiate the recovery program within sixty days after being notified of the situation pursuant to section $469 a$ or within a mutually agreed upon time between himself and the funding or licensing agency. The secretary may provide appropriate compensation for any damages resulting from construction delays or loss of use of nonfederally owned land.

The secretary must issue progress reports on survey activities to the agency responsible for funding or licensing the project [Section 4691-3]. He is also responsible for determining the proper repository for specimens found during the course of such survey activities, and must issue annual reports detailing agency activities of the past year.

Section $469 \mathrm{c}$ provides that a federal agency responsible for a construction project may provide the secretary with funds equally not more than one percent of the total amount appropriated for such project. However, the one percent limitation does not apply for projects involving $\$ 50,000$ or less. The costs of the survey, analysis and publication are considered nonreimbursable project costs.

Sections $469 d-i$ pertain to the protection, preservation and interpretation of the nationally significant values of the wisconsin Continental glaciation, and especially the evidence of such glaciation in the state of wisconsin.

State Nature and Historical Preserve Act - NYECL S 45-0101 et. seq. The goal of New York's Nature and Historic Preservation policy is to preserve and protect lands of natural beauty, wilderness or geological, ecological or historical significance. The policy is 
administered through the State Nature and Historical Preserve Trust. This trust is composed of a Board of Trustees and the Commissioner of Environmental Conservation, who serves as its chairman. The functions of the board include: recommending to the governor and legislature real properties that should be included in the preserve, preparing and submitting an annual report, maintaining an inventory of property dedicated to the preserve, requesting aid from other state agencies when appropriate, and making the rules and regulations necessary to sucessfully administer New York's Nature and Historic Preserve policy. The department may acquire lands when funds are authorized by the legislature and will maintain control over any such properties purchased pursuant to this section. The foregoing laws establish the leadership roles of the Federal and state governments in preserving, restoring and maintaining the prehistoric, historic and cultural resources of the nation and state, respectively. Federal agencies are responsible for administration of cultural properties, the initiation of criteria that assure that federally owned sites, structures, and objects of historical, architectural and archeological significance are preserved, restored and maintained and the institution of procedures that contribute to the preservation and enhancement of non-federally owned sites, structures and objects of historical, architectural and archeological significance. The national policy is administered by the secretary of the Interior through the National Park Service and the state policy by the Board of Trustees of the State Nature and Historical Preserve Trust.

Section 469 of the United states Code expressly applies to any damage or destruction that may result from dam construction, and therefore may be relevant to the Watervliet Reservoir project. A procedure is established for notifying the secretary of the Interior concerning the allocation and nature of the proposed activity and the effect it will have on surrounding terrain, including prehistoric and historic sites. Upon notification that a cultural resource may be disturbed, the secretary is responsible for exercising supervisory control over the initiation, financing and 
recovery of data from the site, and for assuring that significant historical, architectural, and archeological properties are protected and maintained.

Measures for obtaining grants to aid in preservation policies are detailed in Section 470 of the United States Code.

It is to be noted that regulations regarding procedures for effectuating the policies set forth in the foregoing statutes and for assuring compliance with such statutes will have to be consulted prior to execution of this project. 


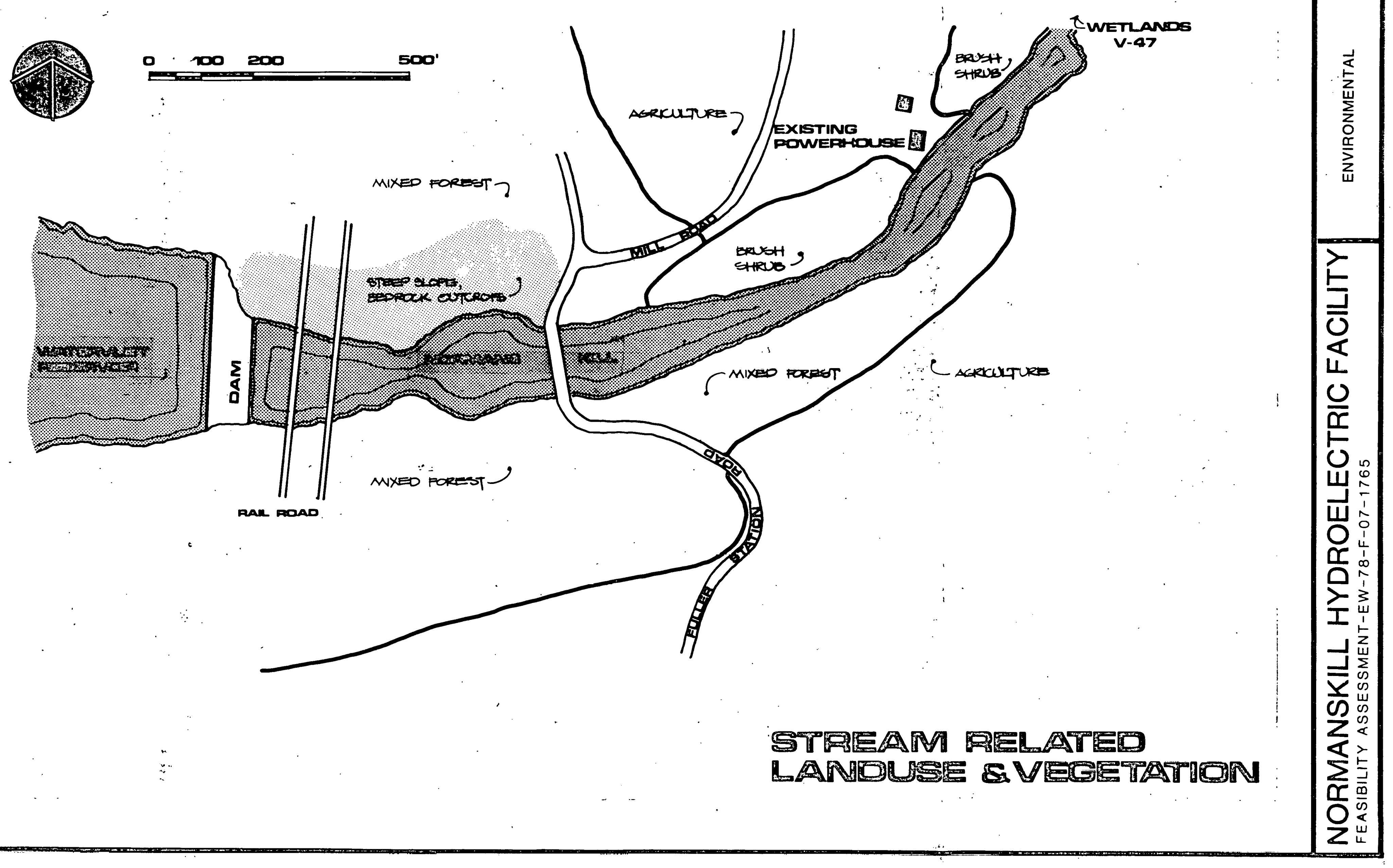




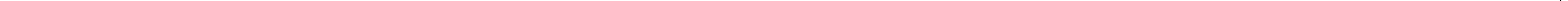





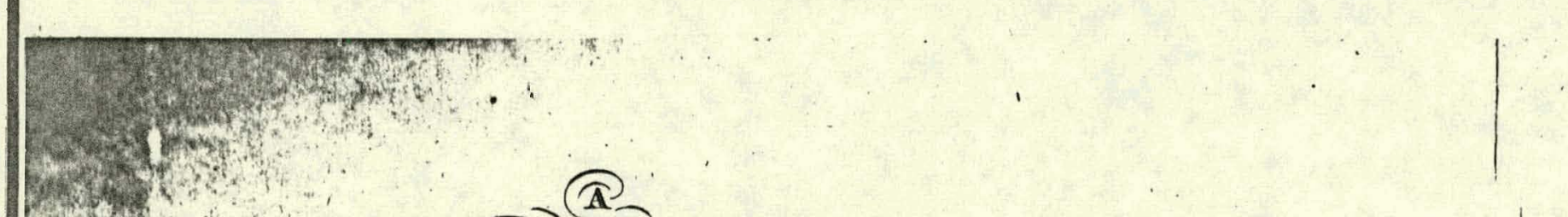

ลิ5

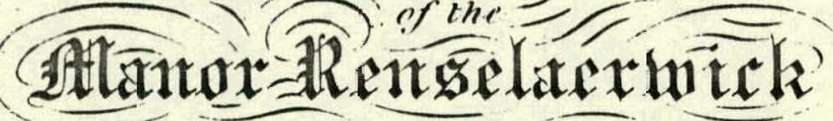
= Surveyed and Ialid Down By a Scale of 100 Chains Tran Inch By $1 \overline{7}(3)$

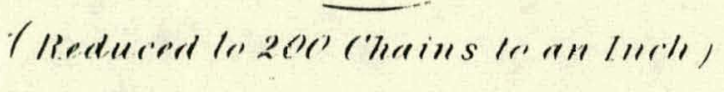
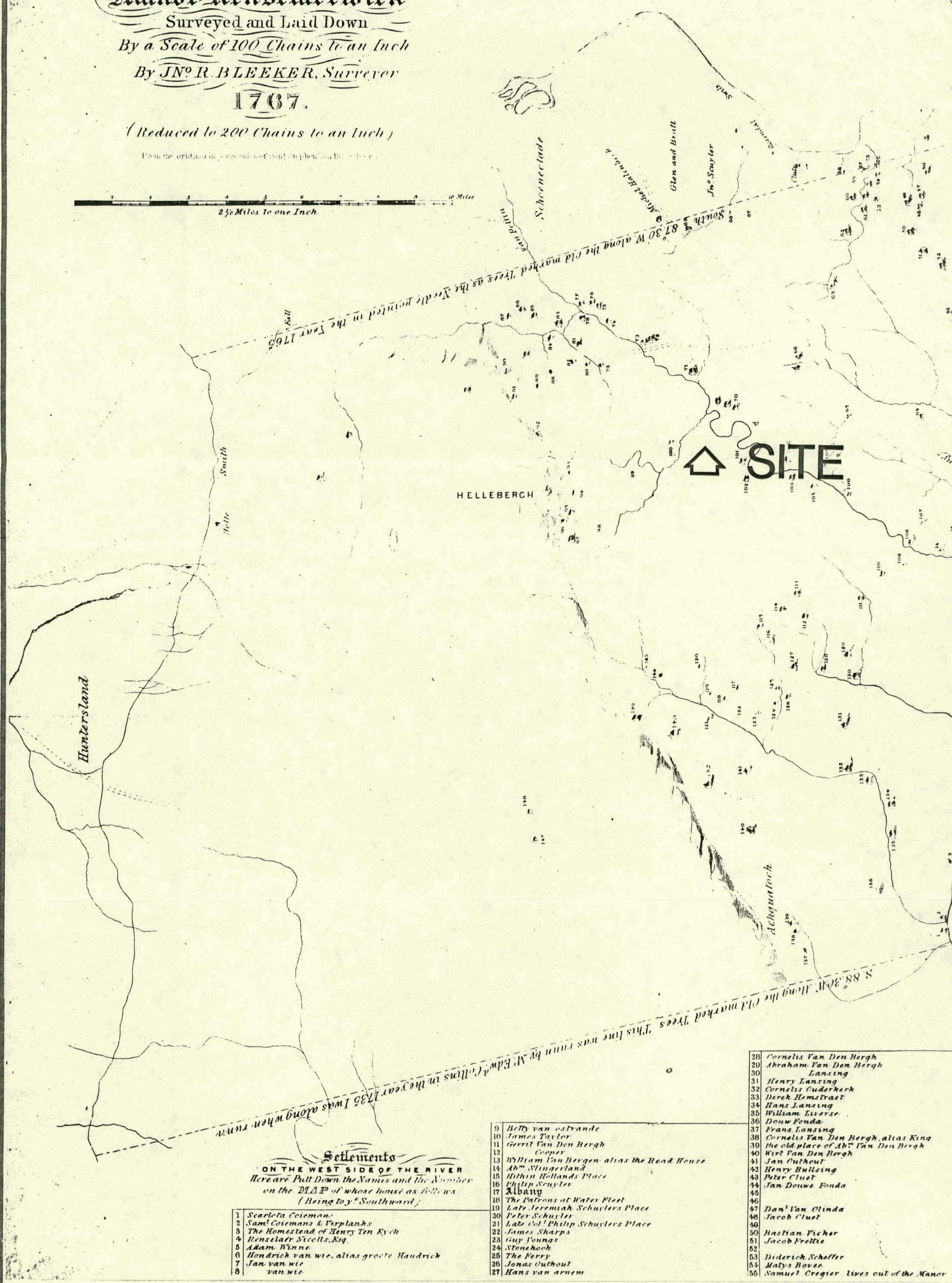
NEW TOPO ATLAS OF

ALBANY \& SCHENECTADY COUNTIES

S.N. \& D.G. BEERS 1866

STONE \& STEWART PUBLISHERS

C.

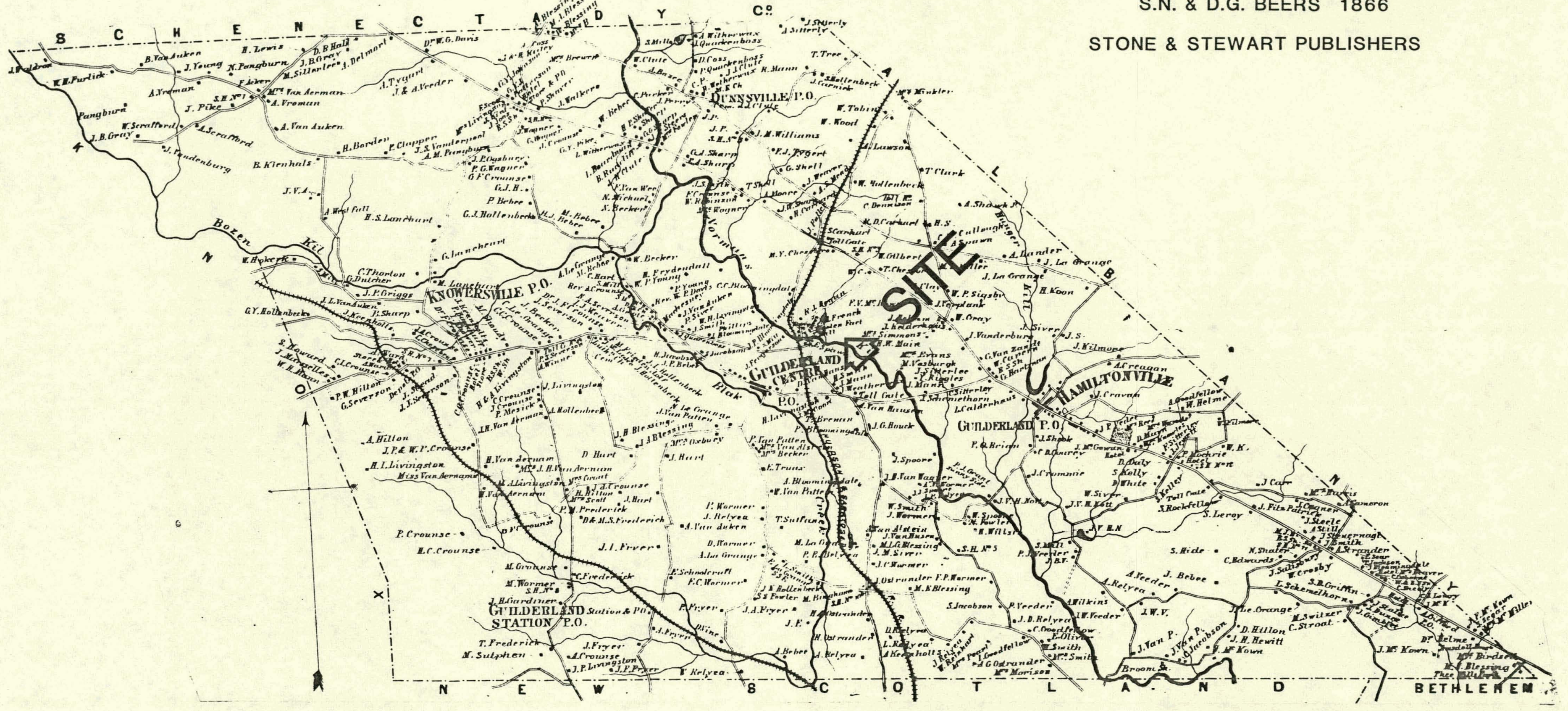




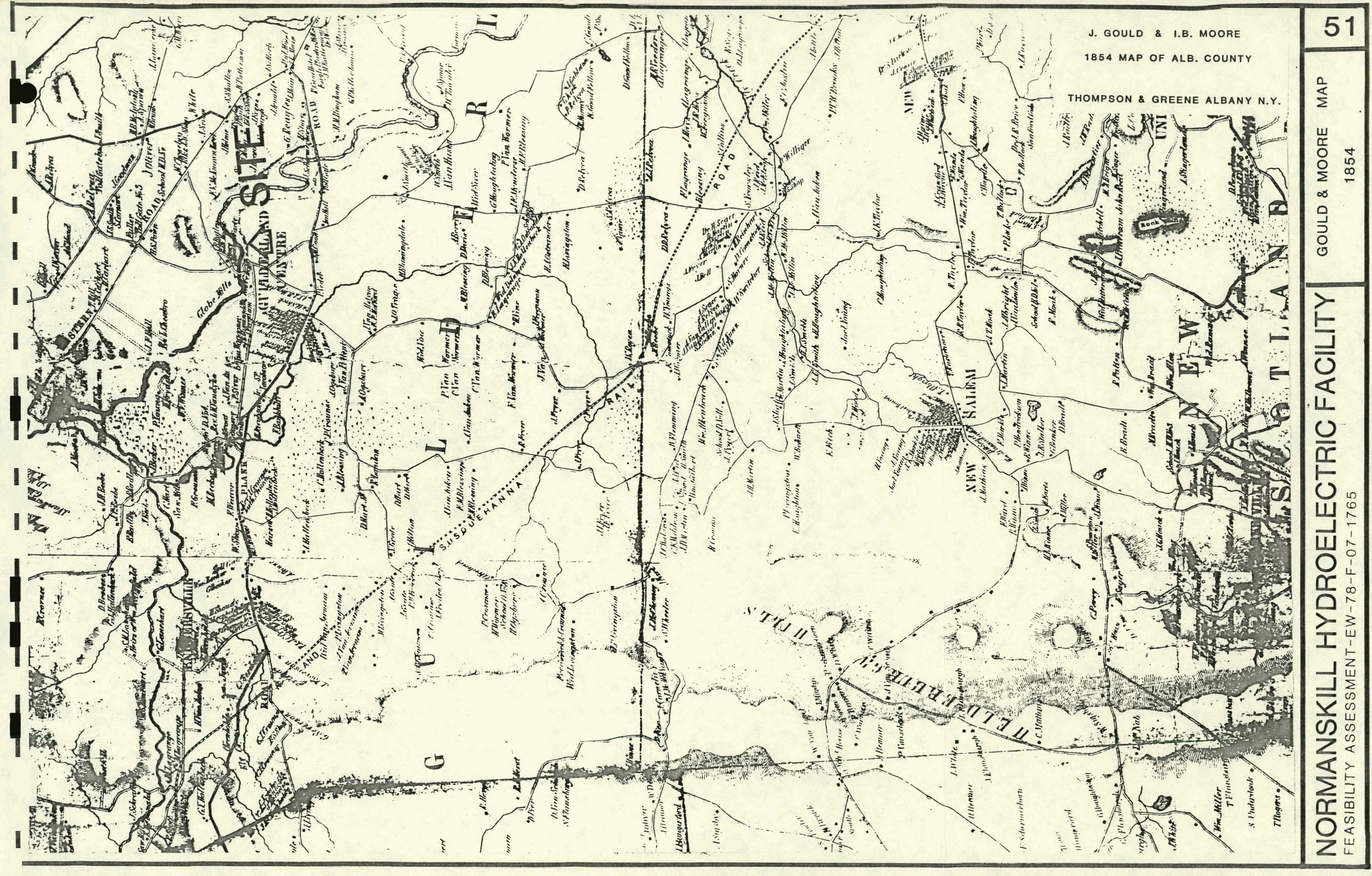




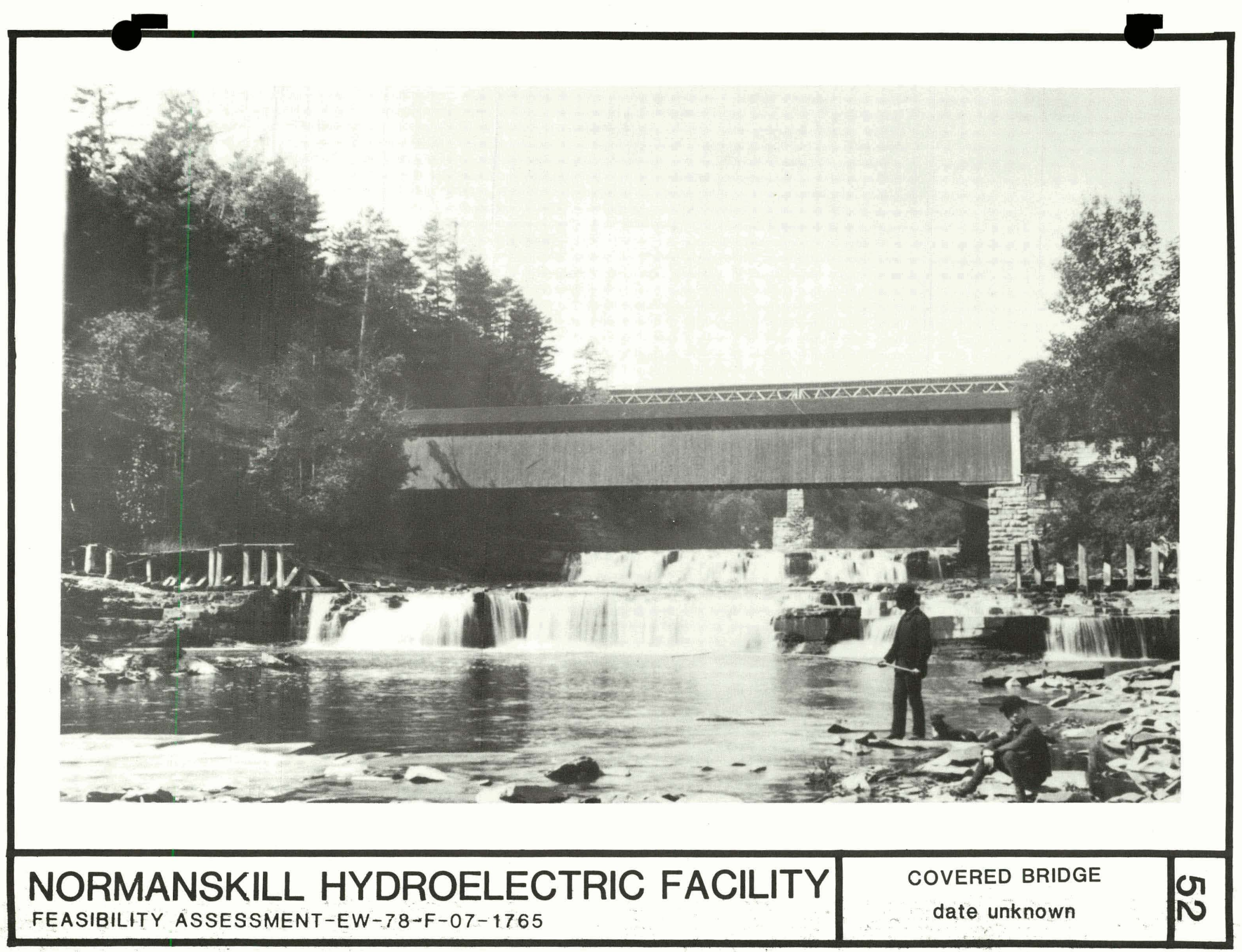




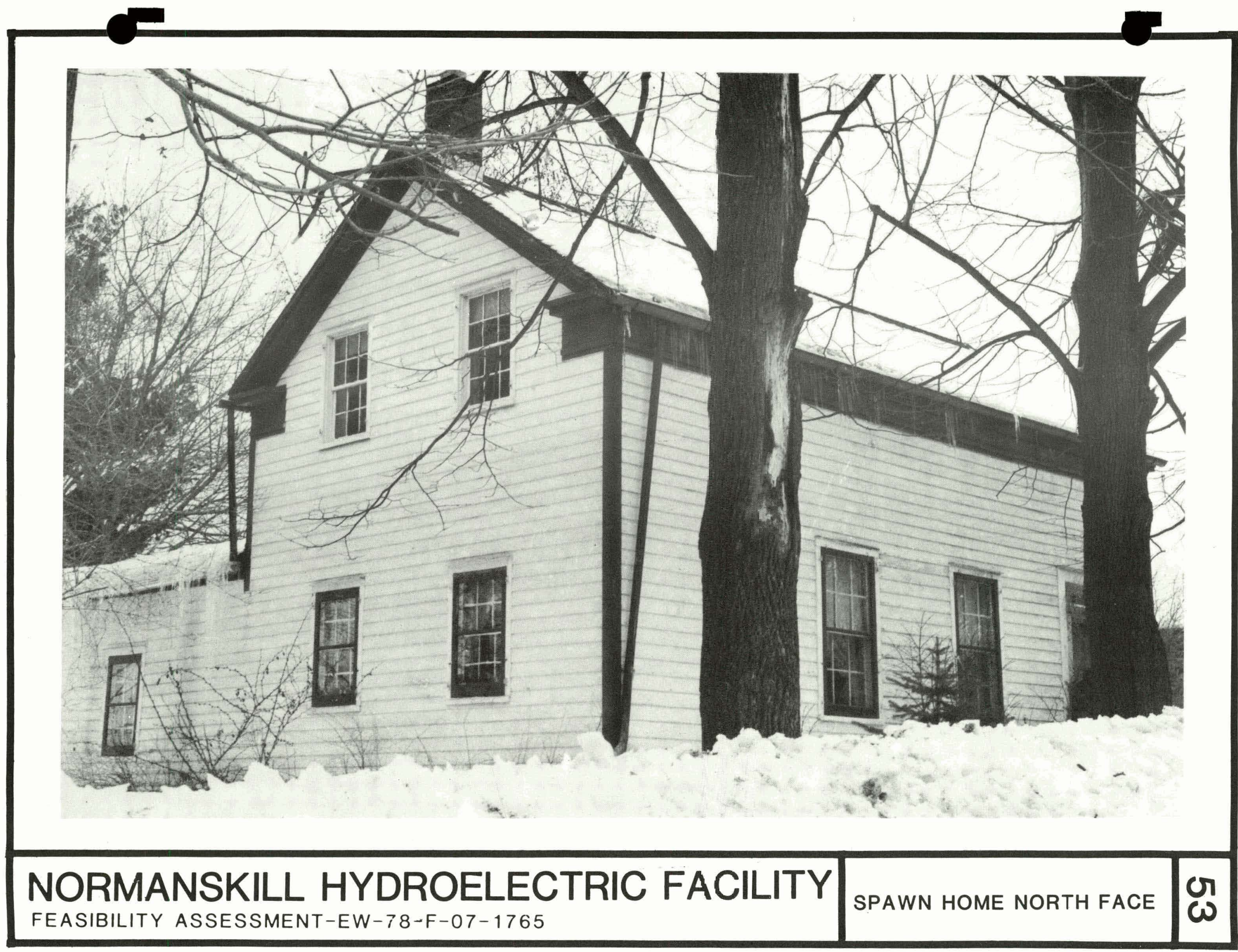




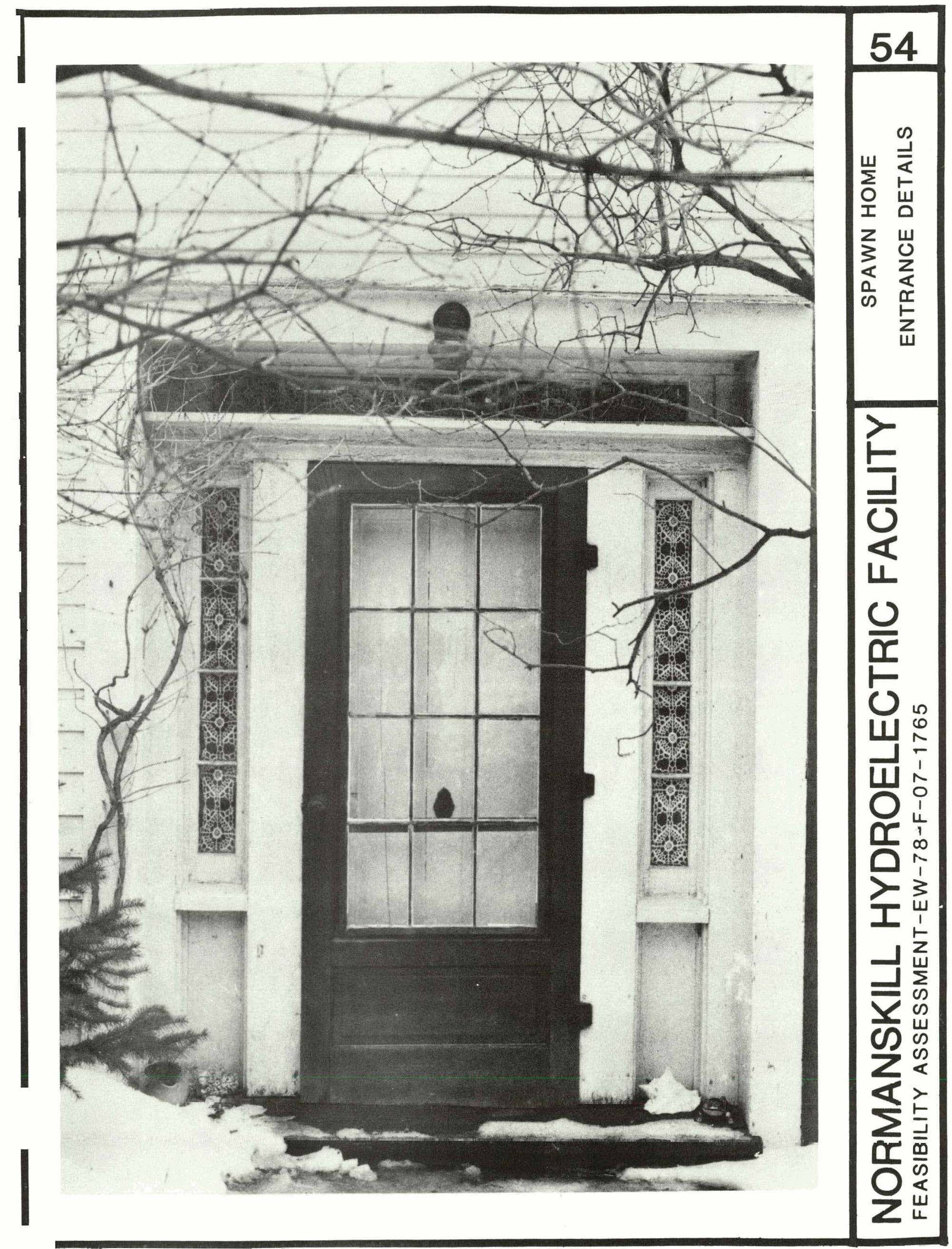




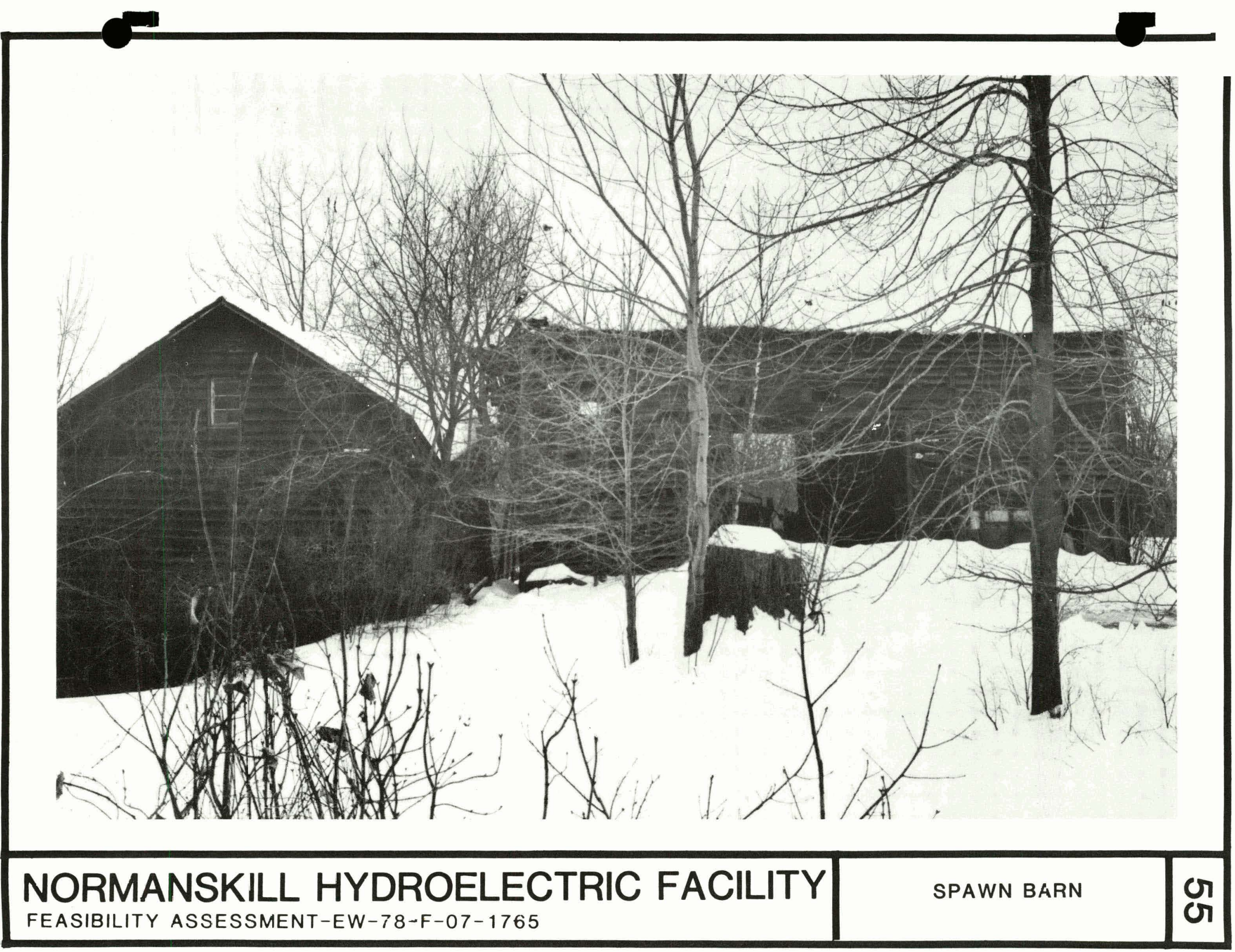




\section{LICENSING AND REGULATORY REQUIREMENTS}

\section{Jurisdiction}

The proposed Normanskill Hydroelectric Facility falls under the aegis of the Federal Energy Regulatory Commission. Discussions with the New York State Public Service Commission and with the New York State Department of Environmental Conservation have determined that any regulation on their part would be subordinate to the Federal Energy Regulatory Commission.

\section{Review Reguirements.}

The New York State Public Service Commission, acting under Public Service Law Section 110[4] Section 66[12] would be required to approve any contractural agreement between the municipal owner of the Normanskill Hydroelectric facility and any purchaser of power [Ref.99].

The Federal Energy Regulatory Commission has determined that the proposed facility will be eligible for short-form licensing. A copy of this determination is on file [Ref. 95]. The FERC has also furnished a list of local, county, state and federal agencies which must participate in the review process prior to submission of an application for a short-form license. These agencies have been requested to estimate time required for their review processes to aid implementation in planning phases of this feasibility analysis.

A list of the nine agencies and the responses follow.

Agency

U.S. Environmental Protection Agency

U.S. Dept. of the Interior

Fish and Wildlife Service
Reply as of $4 / 19 / 79$

no reply

10 days 
U.S. Dept. of the Interior

Heritage and Conservation Service

no reply

U.S. Army Corps of Engineers

two years, if

Section 404 Permit required; if not, uncertain.

U.S. Office of Parks \& Recreation

30 days

NYS Dept. of Environmental Conservation

15-170 days; possibly concurrent FERC review.

NYS Fish \& Wildife Management Board

no review required

NYS Public Service Commission

no reply.

Albany County Board of County Leglslators no reply

\section{Critical Energy Facility Program}

Subsequent to discussions with the above agencies, the President issued Executive Order 12129 of April 05, 1979 [Ref. 105]. This Order directs the office of Management and Budget to establish a system of coordination and deadines for administrative decision-making by Federal agencies. The Program appears at this instance to be an attempt to expedite agency review, simplify and shorten the review process for new non-nuclear energy facilities. It is expected that this order will significantly advance the projected facility on-line date shown in the draft PERT chart, Figure 69. 
Environmental and Cultural Regulatory Requirements

The various environmental and cultural regulatory requirements are discussed in detail in Chapter 6 . 
ECONOMIC ANALYSIS

The Economic Analysis serves to determine the optimum values of those critical parameters which can be shown to be highly reactive or in maximizing the objective.

Several criteria can be set for the objective function. First, the net benefit is the value of savings or earnings by the site developer in pursuing a partiçular project. A second criteria is benefit to cost ratio. An extremely favorable ratio might be shown of a minor power project. Selection on this basis alone, however, might result in a very small net benefit and minimal production of power. Other criteria include rates of return and cash flow profiles.

It was initially assumed that a linear programming model could be developed to optimize certain critical variables such as design flow, machine sizing and type, etc. It was determined early in the marketing phase that the cost function unique to this project was discontinuous due to the different values placed upon the power generated. The most valuable use for power generated is in water supply pumping at the site. The second most valuable use for power generated is for power wheeled across utility lines to City owned facilities. The value in this case is equal to the value of power similarly supplied by the area utility less any wheeling charge extracted by the utility for the transmission service. The least valuable class of power generated is the surplus after the first two demands have been met. The value of surplus or "dump" power is set by the market requirements of Niagara Mohawk Power Corporation. A. fourth and relatively minor value derives from the potential use of the generator during periods of non-generation due to low stream flow. In this mode of operation, the facility would operate as a synchronous condenser supplying power factor correction to the Niagara Mohawk Power Corporation grid. While it is recognized that this service may be of some value in maximizing power transmission capabilities of the utility grid, its economic value is as yet undeterminable: For this reason, a token value, thought to be much: less than the actual value, has been included in the analysis. 
Since the benefit function is discontinuous, optimization of net benefits by normal linear programming methodologies becomes a difficult problem. Consideration was given to developing the methodology using the constrained linear systems (CLS) approach, but this was deemed to be too inflexible and imprecise for the analysis.

The methodology subsequently developed and used was a two-step process. 78 alternatives representing a broad range of site uses, design flows, machine types, configurations costs and benefits were compared and ranked. A net cost comparison was made between all 78 alternatives on the basis of present worth. Following this determination of an optimum configuration of component variables, those costs common to all alternatives were included to define two projects. These optimized projects, were then subjected to the following Economic Feasibility Analysis. The methods of analysis and conventions used follow those of Grant \& Ireson [Ref. 11]. The accounting stance assumed was that of the owner, the City of Watervliet, N. Y.

\section{Selected Projects}

Two alternatives were selected which represented the optimum configuration of many variables. These alternatives, Nos. 1.02 and 8.07, represent two distinct types of projects. The first, Project A, represents a rehabilitation type approach utilizing available civil and mechanical works to their fullest capacity. Project A, which consists of Alternative 1.02 plus other common components would develop 468 of potential hydroelectric capacity at the Normans Kill site. Project $B$ is based upon Alternative 8.07. It represents a new construction approach utilizing only certain existing civil works and develops 688 of potential hydroelectric power. Total project cōsts fui each project type follows. Technical details for each project are depicted on Figure 44 and Figures $41 \mathrm{~A}$ and $41 \mathrm{H}$. A more detailed description of the recommended project will follow in the synopsis/Recommendations. 
Project $A$

1. Intake (from Fig. 44)

2. Penstock (from Fig. 44)

3. Powerhouse (" " ")

4. Switchgear Controls(From Fig. 44)

5. Machine (From " ")

6. Anc. Equipment (From " ")

7. Pump/Control Station Modification

a. Removals

b. Superstructure Rehab.

c.. Security Const.

d. Metals

e. Standby Power

f. Surge Control.

g. Piping

h. Pump Rehab.

i. Telemetry

j. Motor Control

k. Elec. Rehab.

8. Mobil. \& Temp. Services

9. Subtotal Construction Cost

10. Admin., Legal \& Technical Cost

11. Interest during Construction

12. Total Project Cost
$\$$

53,700

68,400

91,200

201,500

257,500

70,300

5,000

30,000

4,000

3,000

30,000

19,125

62,000

15,000

9,000

35,000

20,000

136,600

$1,111,325$

148,200

50,375

$\$ 1,309,900$
Project B

$\$ 276,700$

136,800

162,000

328,100

235,600

70,300

5,000

30,000

4,000

3,000

30,000

19,125

62,000

15,000

9,000

35,000

20,000

61,275

$1,502,900$

177,100

67,200

$\$ 1,747,200$

Determination of Discount Rate and Cost and Benefit Gradients

The discount rate to be used to represent the time value of money for economic studies is the subject of lengthy and involved debate. It involves questions of varying discount rates for public versus private projects, and whether the rate should reflect the nominal or the social opportunity cost of money. A detailed discussion and framework'for such analysis can be found in the references cited. For purposes of our analysis, the following rationale is offered. The City of watervliet's rate for municipal bonding is 6.318 based upon a geometric mean of the past five years' issuance of bonds. We feel that a strong case can be made here for using a discounting rate equal to the actual cost of money to the municipality. However, In curjunction with this rate, it seems appropriate to increment (or decrement) other costs and benefits used in the economic evaluation to more nearly represent the observed and predicted patterns of relative price changes. This convention follows closely the methodologies suggested by the cited 
Reference Nos. 40 and 51. We realize that these observed and predicted values or cost gradients include some component of general inflation or general increase in the nominal cost of goods and services relative to the real (constant) value of the same goods and services. Recognizing then that the discount rate should reflect this general and nominal increase in the time value of money, the problem becomes one of selecting an appropriately higher discount rate. The discount rate suggested in the recent U.S. Department of Energy Program Opportunity Notice for Hydroelectric Demonstration is used in our analysis. It is assumed and understood. that this rate of 8.08 includes an inflationary component over and above the. recognized bare time yield of money [Ref. 104]. We are cognizant of the fact, however, of the varying opinions of economists in selecting an appropriate discount rate. We have included in the Sensitivity Analysis section of this phase, detailed data which can help the reader to visualize the relative effect of varying discount rates.

The observed nominal increases in cost for goods and services in the categories pertinent to this analysis are depicted below.

Calculated Cost Gradients

(Compounded Amount Factors, All Positive)

Period in Years (1978 Base)

\begin{tabular}{lllll}
-30 & -20 & -10 & -5 & $+10 \quad+20$ \\
\hline
\end{tabular}

Consumer Price Index

(CPI)

$$
3.258 \quad 3.758 \quad 6.08
$$

Wholesale Price Index

(SPI)

$$
3.258 \quad 3.58 \quad 5.758
$$

Electrical Machinery

$$
4.258 \quad 1.758 \quad 6.08
$$

Labor (Watervliet Múicipal)

$.5 .758 \quad 6.58$

Electrical power

Wholesale U.S.)

$5.258 \quad .7: 58 \quad 16.08$

Electrical Power(NMPC)

6.089 .08


Electrical Power Pro-

jections N.Y.S. Energy

Office (by NMPC)

$1.8 \% 0.0 \%$

Natural Gas Cost

Projection

NYS Energy office

(by NMPC)

$4.2 \%$

Following the convention of [Ref. 51], we have elected to use nominal changes in cost for our analysis. We belive that the cost gradients selected are conservative in range. It is noted that the gradients used are based upon the historical record and include some inflationary component. The determination of an inflationary component by using the U.S. Department of Commerce Consumer or Wholesale Price Index to correct the observed gradients is impractical. The CPI or WPI is a relative index based upon a representative sample of market place goods and services, whereas the inflationary cost increases for specific goods and services varies for different products. Use of a general national index of costs for a disaggregated sample of goods and services on a local or microeconomic level is inappropriate.

In summary, our approach is to use a discount rate which knowingly includes some factor for assumed long-term general inflation and to use nominal cost gradients based upon the best observed and most appropriate data available to the City of Watervliet, New York. As previously mentioned, because of the wide range of opinions among economists in determining these gradients, we have included sufficient data in the sensitivity Analysis section to visualize the impact of making alternative choices.

Present Worth Analysis

The present worth of the baseline condition and Projects $A$ and $B$ have been calculated, representing cash outflows as negative numbers. The factors used in the analysis were:

a. 50-year analysis period.

h. 8.0\% discount rate. 
c. Capital costs incurred at first year.

d. Labor cost gradient 5.8\% per annum.

e. Operation \& maintenance cost gradient $4.0 \%$ per annum.

f. Electrical energy cost gradient $7.0 \%$ per annum.

\section{Present Worth Analysis}

\section{Baseline Project A Project B}

P.W. Capital Cost $\$-407,000$

P.W. Labor $\quad-3,454,437$

P.W. Power - 71,636

P.W. O\&M - 187, 513

P.W. Energy Credits $\frac{0}{\$-4,120,586}$

$$
\$-1,309,900
$$

- 575,740

- 616,863

$-\quad 997,130$

$\frac{+2,367,957}{\$-1,131,676}$
$\$-1,747,200$

- 575,740

- 139,293

$-1,213,322$

$\$ \frac{+2,857,466}{818,089}$

A further discussion of present worths follows under Benefit Cost Ratio and Cash Flow.

Benefit Cost Ratio

The optimum configuration program (OCP) aided in the selection of the best configuration among several possible alternative selections of hydroelectric generating equipment. Alternatives Nos. 1.02 and 8.07 were tentatively selected as optimum configurations for generation equipment. Two separate alternatives were selected because they were not comparable on the same economic basis. Alternative No. 1.02 involves the reuse of certain existing equipment which for subjective reasons may be unacceptable in spite of being less expensive. These configurations have been incorporated into projects which have been designated as Project $A$ and Project $B$, respectively. The feasibility of Project $A$ or Project $B$ can be investigated by determining the benefit-cost ratio of the incremental investment compared to a baseline condition. Our baseline accounting stance is that of operating the existing water pumping station in its present configuration which currently utilizes some mechancial hydropower to operate water supply pumps. The comparison period is taken to be 50 years or the projected life 
of the equipment for Projects $A$ and $B$. The existing facility requires some immediate investment to be able to continue in its present capacity for a comparable 50-year period.

The benefit-cost ratio analysis is depicted on Figure 56. A period of 50 years and compound annual cost increases for labor, energy, and operation and maintenance of $5.8 \%, 7.0 \%$, and $4.0 \%$, respectively are shown. This benefit-cost ratio is based upon the present worth over the entire service period. The first or benefit portion includes the benefits accruing to the city as reduction in total power cost due to power which can be produced in excess of that which will be used to operate the electric pumps. The value of this credit in the base year can be taken from Figure $30 \mathrm{~A}$ and is the total of the credits shown for "city use", "excess", and "power factor" correction. The second benefit item is the reduction in labor costs resulting from automation of the pumping station which will operate on electric power in contrast to the labor intensive existing baseline situation. The present worth of the future benefits is computed for each year of the service period and summed for projects $A$ and $B$. on Figure 56-line 5 .

Items required to determine the present worth of additional costs from implementing the projects are computed and displayed as lines 6-11 on Figure 56. The first such item is capital cost. For analysis period less than 50 years, credit is taken for a present worth of a future residual value which is computed by assuming that the basic value of the comparable new facility increases at a rate equal to the average of the energy, labor, and operation and maintenance cost gradients (5.6\%), but because of its age, it is simultaneously reduced on the ratio of its age to its 50-year 1 ife. This future residual value is then discounted to present worth using the stated discount rate $(8.0 \%)$. The second cost items are power to operate the pumps during low streamflow periods when turbines cannot produce electricity. The baseline condition assumes purchase of power for pumping for approximately 14 days per year at the assumed rate of 5.3 cents per kilowatt hour in the base year. Under projects $A$ and $B$, pumping costs in the base year are calculated to 
be $\$ 45,460$ ( 365 days at $98 \mathrm{kw}$ at 5.3 cents per $\mathrm{kwh}$ ) less the amount shown under "site use" power credits in Figure 30A. The third cost item is Operation and Maintenance, the present worths of which is computed by escalating to future values in each year of the service period using the gradients shown, discounting them back to present worth at the indicated discount rate and summing them as in the benefits section.

In accordance with Grant and Ireson [Ref. 11], benefit cost ratio on the incremental investment is calculated as the incremental increase in benefits from the base Iine to Projects $A$ or $B$ on. Iine 5 divided by the corresponding incremental increase in costs on line 12. Labor costs are not included in line 12 costs since the substantial reduction in labor costs have been taken as one of the major benefits and we are interested in determining the ratio of incremental benefit to incremental cost increases.

We feel that it is illogical to consider the implementation of the hydroelectric power generating facility without simultaneously. upgrading the city's pumping station at the site. Although it is not shown in Figure 56, investigation was made of the relative benefit cost ratio of the incremental portions of the projects to upgrade the pumping station with its resulting labor saving benefits. The benefit cost ratio of this increment relative to the baseline was 1:81. The incremental benefit cost ratio for the further investment to add hydroelectric power generation resulting in the full Project $A$ or $B$ was incrementally 3.56 and 3.40 , respectively.

In all cases, a benefit cost ratio greater than one indicates that an. investment will bring a return greater than the investment over the study period.

\section{Cash Flow}

In order to permit the analysis of anticipated future cash flow associated with the projects, a cash flow model was developed which computes the annual costs for any specified period. Capital costs are indicated as a single expense at year zero while labor, 
power and operation and maintenance costs are shown as annual costs increasing from a base year value at a compound annual rate in this example equal to $5.8 \%, 7.0 \%$ and $4.0 \%$, respectively, as used in the benefit cost program. The net cash flow for any year is the sum of all previous columns using the convention that a negative value is a cash outflow. The last or cumulative present worth column shows the base year cumulative present worth of all years net cash flow through that given year, in this case using an 88 discount rate.

A projected 50-year cash flow for projects $\dot{A}$ and $B$ are included as Figures 58. A similar cash flow is shown for the corresponding baseline condition assuming that substantial investment is. immediately required to enable it to operate for the 50 year period. It should be noted that the operation of the pumping station in each case is essential to the welfare of the city of watervliet. It can be seen that the baseline condition shows a lesser capital expenditure and operation and maintenance cost, a much greater outflow for labor cost, and none of the large credits for power production available under projects A or B. By comparing the cash flows for Projects $A$ and $B$ with the baseline cash flow, it can be seen that the increased investment in Projects $A$ or $B$ will'be repaid by the tenth or thirteenth years, respectively when the cumulative present worth cash outflow for either project becomes less than the corresponding item under the baseline condition. The total present worth of the 50-year series for each column is summarized on the bottom line of each of the displays in Figure 58.

\section{Delta Cash Flow}

To facilitate making the comparison between the cash flow for the proposed project and that for the baseline condition, the Delta Cash Flow display of Figure 58 . shows the net change in cash flow resulting from investing in Project $A$ or $B$. Figure 58 shows these net changes for Projects $A$ or $B$ utilizing the same assumptions as were used in the preceding Cash Flow description. The convention used shows increases in cost as negative numbers and cost reductions as positive numbers. It can be seen again that the capital 
investment and added incremental operation and maintenance cost in Projects $A$ and $B$ is more than offset by gains. in labor savings and power credits so that after 10 or 13 years respectively the investment has turned into a net benefit compared to the baseline condition.

\section{Discounted Cash Flow}

The computed benefit cost ratios for the proposed projects demonstrate that investment in the projects merits consideration. The owner must compare that potential investment with other possible expenditures to decide whether that project warrants his investment. The owner may have other less attractive projects for which he may be obligated to spend his available capital, and cash flow problems may prevent him from making the investment.

For those reasons, and to help the owner to evaluate the relative merits of an investment in Project $A$ or $B$, we have analyzed the investment in another way by showing the calculated rate of return for the incremental project relative to the baseline condition. A variation on the Delta Cash Flow was used in Figure 57 in which the change in net cash flow for the study period was obtained, but using an itterative procedure, the internal rate of return was determined. This computed rate which, when used to represent the time value of the investor's money or interest rate for discounting future cash values to present worth, causes the project to break even. The rates so computed for projects $A$ and $B$ are $18.91 \%$ and $16.24 \%$, respectively. This means that an investment in the project will be recovered over the service period, using the stated assumptions, when the value of money to the investor is as high as the calculated rate of return. The bottom line in each display is present worth of the 50-year series when discounted using the computed internal rate of return. The total net discounted cash flow is zero dollars. The internal rate of return on an an incremental investment is one more measure which the owner can utilize in trying to decide among competing investments which one is most advantageous for him. 
Subjective Considerations

Economic analysis indicates that both Project $A$ and Project $B$ merit. implementation. However, consideration must be given to non-quantitative indicators such as environmental impact, cultural resource impact, licensing constraints and other intangibles. Rather than attaching economic values to allow analysis in a supposedy "analytical" fashion; a simple listing of pertinent subjective factors and their relative impact on both projects is presented. Some admittedly subjective judgments are included in the ratings.

Relative Impacts of

other Subjective Considerations

Relative impact scale:

$$
\begin{aligned}
0 & =\text { beneficial or easy } \\
5 & =\text { minimal or normal } \\
10 & =\text { adverse or difficult }
\end{aligned}
$$

Project $A \quad$ Project $B$

Environmental

Reservoir related

5

Stream related ( $B$ is seen as having beneficial effects on dissolved oxygen levels)

Recreational impact

other impacts(drinking water)

Cultural \& Archaeological

Historic site disruption

Iicensing

Approvable design

Review delays

9

9

5

Construction Related

Lead time required

Disruption of exist. facilities

Weather (streamflow) sensitive

Energy Significance 
Implementation

Easement acquisition

Power purchase agreement

with utility

Impact on utility revenues

Sensitivity Analysis

Extensive research was performed as part of the analysis to determine the economic assumptions which could best represent the owner's true point of economic view. After considerable effort we concluded that it was impossible to select economic rates which were acceptable to a consensus of authorities. We therefore have presented the various computational output material using consistent assumptions which we felt were most reasonable from the accounting stance of the City of Watervliet. Expecting that each reviewer will wish to impose his own assumptions on the economic analysis, we have computed and displayed a series of data matrices showing interrelationships among several of the economic variables for the proposed and recommended Project B. This data has been plotted as an indicator of the response of benefits versus several of the most important and controversial variables. Each reviewer can therefore assess the sensitivity of a particular benefit to a particular change or error in economic variables. The most important and controversial factors were those pertaining to cost escalation gradients for labor and energy, particularly in relation to the choice of a discount rate and period of years for the analysis. Therefore, included as Figures 59-67 are displays showing the shape of the response surfaces for both the present worth of future net benefits and the benefit-cost ratio computed for the incremental investment in Project $B$ relative to the baseline condition. The sensitivity, of the value of the resulting benefits to possible errors in capital cost, are likewise shown. Except where a range of values for certain of the economic variables have been substituted to investigate sensitivity to error, the computation in this and previous sections are based on the following summary of values: 
1. Capital Costs - See Benefit-Cost Ratio (Figure 56) and Present worth Analysis

2. Present worth of Future Residual Facility Value is equal to the capital cost escalated, to facilitate automatic computation, at an annual rate equal to the average of labor, energy, and $O \& M$ escalation gradients (5.6\% at chosen values), prorated to its remaining life, and discounted to present worth using the discount rate.

3. Energy Rates - See Figure 30A; "Annual Power Cost and Credit Summary".

4. Base Year Power Costs - See Benefit-Cost Ratio (Fig. 56). and Present. Worth Analysis

5. Base Year Power Credit-See Benefit-Cost Ratio (Fig. 56). and Present Worth Analysis

6. Base Year O \& M Cost - See Benefit-Cost Ratio (Fig. 56). and Present Worth Analysis

7. Base Year Labor Costs for the baseline or Project $B$ condition are based on five or one man, respectively, at $\$ 18,629$ per year.

8. Energy Values Increase at 7.08 per Year Compounded.

9. Labor Values Increase at 5.88 per Year Compounded.

10. O \& Values Increase at 4.08 per Year Compounded.

11. Discount Rate equals $8.0 \%$ per Year Compounded.

12. Analysis Period is 50 Years.

Economic Criteria for Incremental projects

In completing this feasibility assessment, several aspects of site development of possible economic significance became apparent. These aspects have been given a cursory examination as Incremental Projects. The criteria used for this preliminary evaluation was benefit/cost ratio based upon a ratio of present worth net benefits to cost. Gradient factors used are those established in previous sections. In addition, value gradients for natural gas, unfinished water, municipal ice rink rental and coal are $4.28,1.08,2.08$ and 4.0\%, respectively. A 50-year analysis period is used with a 8.08 discount rate. Analysis of Incremental Projects follows: 


\section{Incremental Project I - Heating Systems Conversions to Electric}

Assume: Conversion of dual fuel (gas/electric) space heating systems at certain municipal buildings to electric to utilize surplus electric power produced.

Facility

Senior Citizens Center City Hall

City Garage

Water Filtration Plant Civic Center

Firehouse
Current Annual

Natural Gas Use (CCF)

4,942

17,642

11,774

15,675

1,202

13,628
Annual Cost

$\$ 1,180$

4,410

2,940

3,920

300

$\frac{3,400}{\$ 16,150}$

Benefits: 1. Natural Gas Savings (608 reduction in use)

2. Disbenefit (Loss of Dump Power) at $0.9 \not \mathrm{KWH}$

3. Net Benefit (Year 1)

4. $\mathrm{PW}-\mathrm{B}$

Costs: $\quad$ 1. Capital Cost

2. O \& M Cost

3. Power Wheeling Charge @ $0.4 \not \mathrm{KWH}$

4. Net Cost (Year 1)

5. $\mathrm{PW}-\mathrm{C}$

$\mathrm{B} / \mathrm{C}=\frac{166,079}{42,060}=3.9$

Incremental Project 2 - Extending Ice Rink Season

Assume: Extension of ice rink season one month utilizing surplus electric power to maintain ice.

Benefits: 1 . Sale of Rink Time

108 hrs. e $\$ 30 . / \mathrm{hr}$.

2. Disbenefit-Loss

of Dump Power @ $0.9 \not \mathrm{KWH}$

3. Net Benefit (Year 1)

4. $\mathrm{PW}-\mathrm{B}$

Costs: 1. Labor at Rink

2. $O \& M$

3. Power wheeling Charge @ $0.4 \not \subset \mathrm{KWH}$

4. Net Cost

5. $\mathrm{PW}-\mathrm{C}$

\begin{tabular}{rr}
$\$$ & 486 \\
$\$$ & 200 \\
$\$$ & 540 \\
\hline$\$$ & 1,226 \\
$\$$ & 19,429
\end{tabular}

$\mathrm{B} / \mathrm{C}=\frac{3,562}{19,429}=0.18$ 


\section{Incremental Project 3 - Coal Heat Conversion}

Assume: Conversion of coal fired space heat at French's Mills Pump/Control station to electric heat.

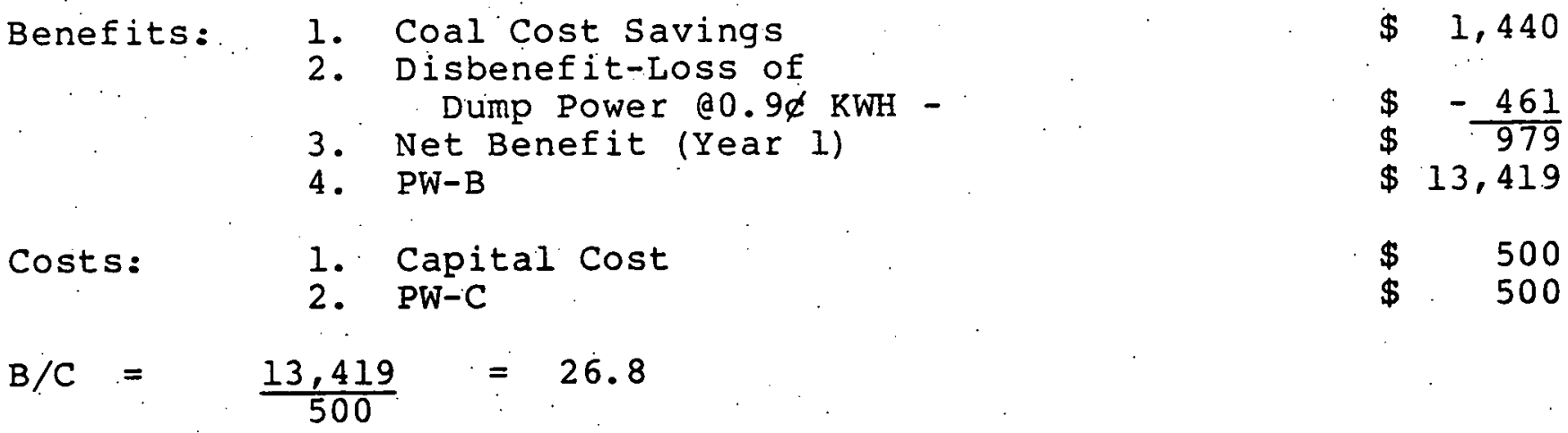

Incremental Project 4 - Bascule Gate

Assume: Installation of Bascule Gate atop dam to increase available head for power and increase water supply yield.

a. Power

Benefits: $1 . \quad$ Increased hydroelectric

$$
\text { 2. } \mathrm{PW}-\mathrm{B}
$$

$\$ 14,789$

$\$ 588,500$

$\$ 2,255,000$

$\$ 2,255,000$

$B / C=\frac{588,500}{2,255,000}=0.26$

b. Water Yield

Benefits: $1 . \quad$ Addition $10 \mathrm{mgd}$ Yield

Capital Costs

$\mathrm{PW}-\mathrm{C}$

$$
\$ 2,255,000
$$




\section{Conclusions}

Based upon the economic criteria preceding and other subjective considerations, the recommended course of action is implementation of Project $B$. Within the range of incremental projects, only Incremental projects 1 and 3 are recommended with their adoption delayed until completion of the hydroelectric project. 
ECONOMIC COMPARISON OF PROJECTS A\&B TO BASELINE ALTERNATIVE

\section{BENEFIT/COST RATIO}

NORMANS KILL HYIRO-ELECTRTC FEASIBILITY STUMY BENEFIT / COST RATIO PROGRAM (BCP)

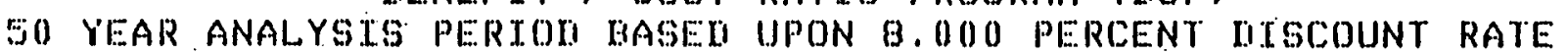

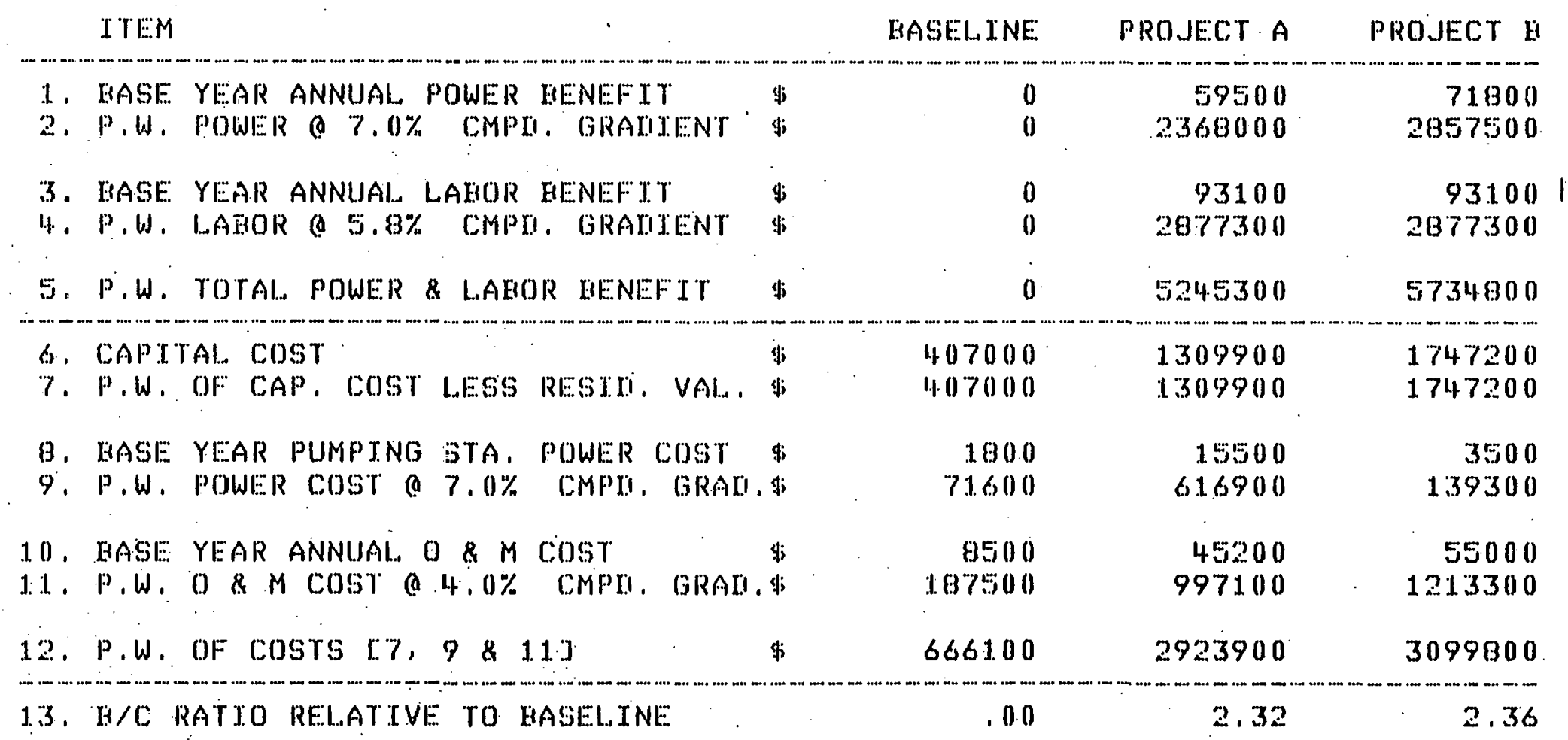


ECONOMIC COMPARISON OF PROJECTS A\&B

TO BASELINE ALTERNATIVE

INTERNAL RATE OF RETURN

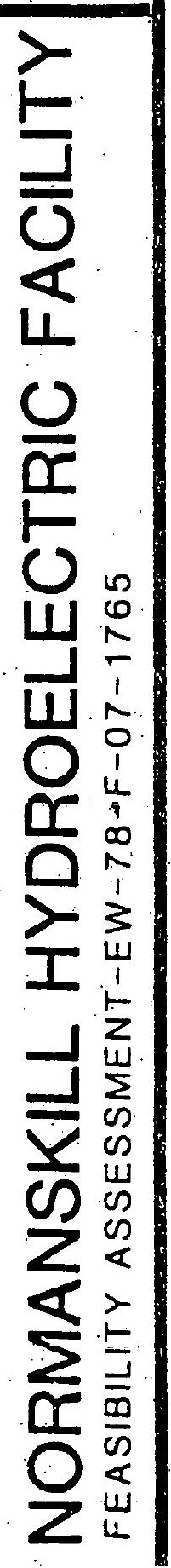


NORMANS KILL HYIIRO-ELECTRIC FEASIBILITY STUMY

IIISCOOUNTEII CASH FLOW PROGRAM (CFPS)

PROJECT A COMPAREII TO HASELITHE ALTERNATIVE

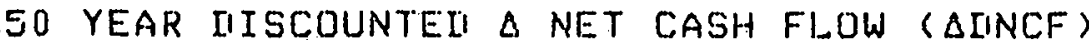

COMPUTEII INTERTAL RATE OF RETURN EQUAL TO 18.91419 PERCENT

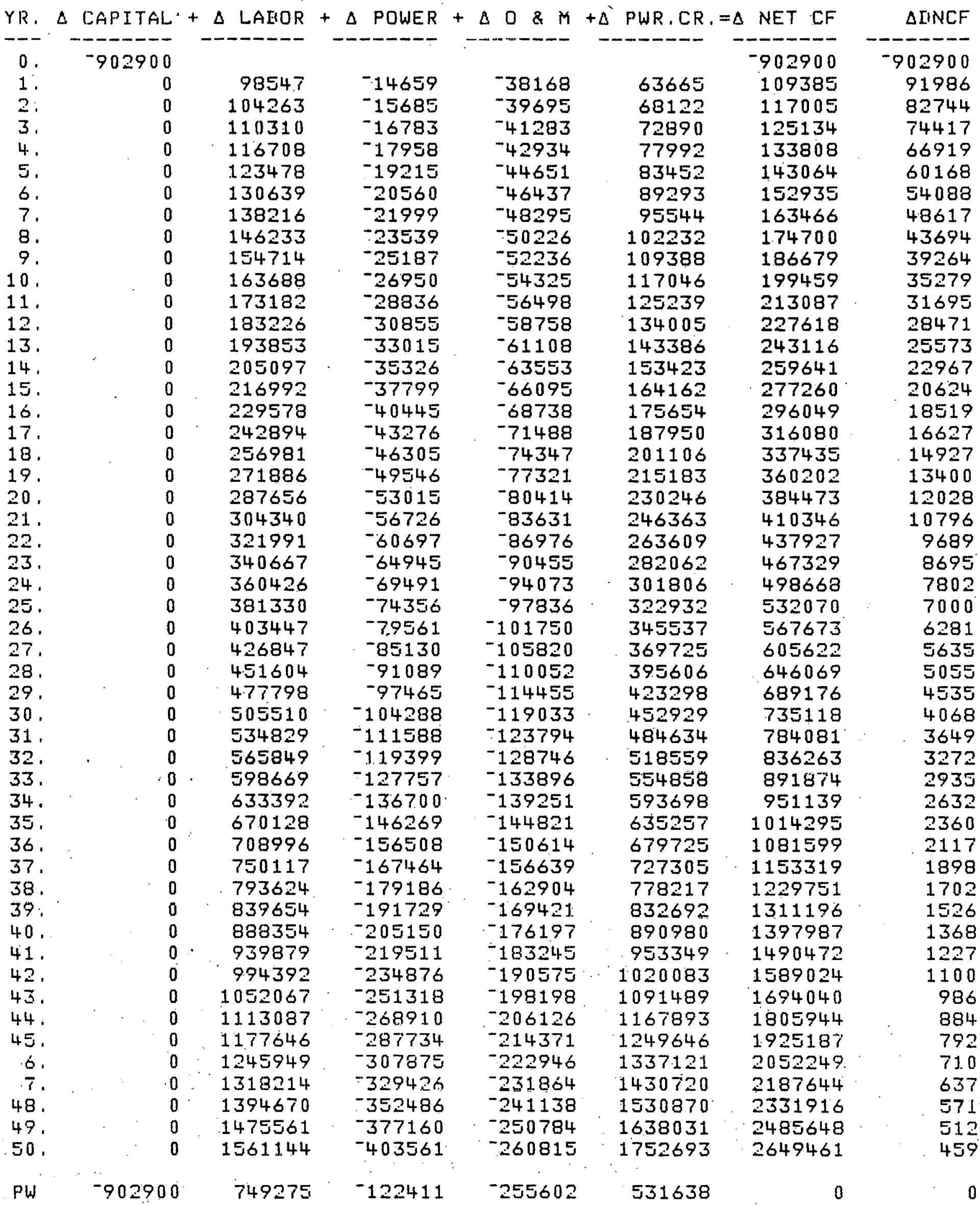


WORHAISS KILL HYIIRO-ELECTRIC FEASIBILITY STUIYY

IISCOUNTEI CASH FLOW PROGRAM (CFP2)

PROJECT H COMPAREI TO HASELINE ALTERNATIVE

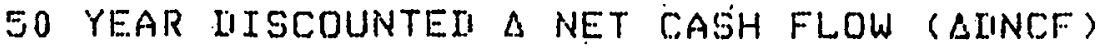

COMPUTEI INTERNAL RATE OF RETUIRN EQUAL TO 16.24351 PERCENT

YR. $\triangle$ CAPITAL + $\triangle$ LAEOR + $\triangle$ POWER + $\triangle O \& M+\triangle$ PWR.CR $=\triangle$ NET CF

$\begin{array}{rr}- & -1340200 \\ 0 . & 0 \\ 1 . & 0 \\ 2 . & 0 \\ 3 . & 0 \\ 4 . & 0 \\ 5 . & 0 \\ 6 . & 0 \\ 7 . & 0 \\ 8 . & 0 \\ 9 . & 0\end{array}$

10.

11 .

12.

13.

14.

15.

16.

17.

18.

19.

20.

21.

22.

23.

24.

25.

26.

27.

28.

29.

30.

31.

32.

33.

34.

35.

36 .

37.

38.

39.

40.

41.

42.

43.

44.

15.

6.

7.

48.

49.

50 .
104263

110310

116708

123478

130639

138216

146233

154714

163688

173182

183226

193853

205097

216992

229578

242894

256981

271886

287656

304340

321991

340667

360426

381330

403447

426847

451604

477798

505510

534829

565849

598669 .

633392

670128

708996

750117

793624

839654

888354

739879

994392

1052067

1113087

1177646

1245947

1318214

1394670

1475561

1561144

\section{7}

$-1946$

$-2083$

$-2228$

- 2384

- 2551

$-2730$

$-2921$

$-3125$

$-3344$

$-3578$

$-3829$

$-4097$

$-4384$

$-4690$

$-5019$

$-5370$

$-5746$

$-6148$

$-6578$

$-7039$

$-7532$

$-8059$

$-8623$

$-9227$

$-9872$

$-10564$

$-11303$

$-12094$

$-12941$

$-13847$

$-14816$

$-15853$

$-16963$

$-18150$

$-19421$

$-20780$

$-22235$

$-23791$

$-25457$

$-27239$

$-29145$

$-31185$

$-33368$

$-35704$

$-38203$

$-40870$

$-43739$

$-46801$

$-50077$
$-1819$ $-\cdots-\cdots$

$-48360$

$-50294$

$-52306$

$-54398$

$-56574$

$-58837$

$-61191$

$-63638$

$-66184$

$-68831$

$-71585$

$-74448$

$-77426$

$-80523$

$-83744$

$-87094$

$-90577$

$-94200$

$-97968$

$-101887$

$-105963$

$-110201$

$-114609$

$-119194$

$-123961$

$-128920$

$-134077$

$-139440$

$-145017$

$-150818$

$-156851$

$-163125$

$-169650$

$-176436$

$-183493$

$-190833$

$-198466$

$-206405$

$-214661$

$-223247$

$-232177$

$-241464$

$-251123$

- 261168

$-271615$

$-282479$

$-293778$

$-305530$

$-317751$

$-330461$
- - - - -

76826

82204

87958

94115

100703

107752

115295

1.23366

132001

141241

151128

161707

173027

185139

198098

211965

226803

242679

259667 .

277843

297292

318103

340370

364176

38.9690

416968

446156

477387

510804

546560

584819

625756

669559

716429

766579

820239

877656

939092

1004828

1075166

1150428

1230958

1317125

1.409323

1507976

1613534

1726482

1847335

1976649

2115014
- - - - - -

$-1340200$

125194

134227

143879

154197

165223

177003

189590

203040

217406

232754

249147

266656

285357

305329

326656

349430

373750

399714

427437

457034

488630

522361

558369

596805

637832

681623

728362

778248

831491

888311

948950

1013664 


\section{CASH FLOWS:}

BASELINE

PROJECT A

PROJECT B

PROJECT A minUS BASELINE

PROJECT B minUS BASELINE

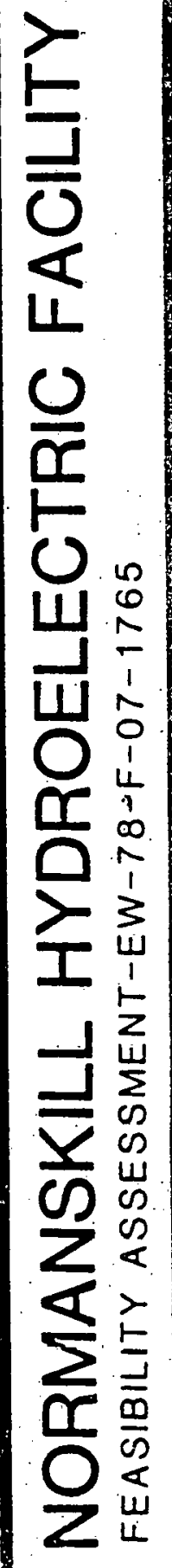


NORMANS KILL HYIIRO-ELECTRIC FEASIBILITY STUIYY

CASH FLOW PROGRAM (CFF1)

BASEL INE

SO YEAR ANALYSIS PERIOI ASSUMING 8.000 PERCENT IIISCOUNT RATE:

\begin{tabular}{|c|c|c|c|c|c|c|c|c|}
\hline २. & CAPITAL & LABOR & POWER & $+0 \& H$ & $+P W R, C$ & & NET & CUM. \\
\hline-- & $---m--\infty$ & ------- & ----- & ---- & & & $\cdots-\cdots$ & -1 \\
\hline 0 & -407000 & & & & & & -407000 & -40700 \\
\hline 1 & 0 & -118257 & -.1926 & -8840 & & 0 & -129023 & -52646 \\
\hline 2 & 0 & -125116 & -2061 & -9194 & & 0 & -136371 & -64338 \\
\hline 3 & 0 & -132373 & -2205 & -9561 & & 0 & -144139 & -7578 \\
\hline 4 & 0 & -140050 & -2359 & -9944 & & 0 & -152353 & -86978 \\
\hline 5. & 0 & -148173 & -2525 & -10342 & & 0 & -161040 & -97938 \\
\hline 6. & 0 & -156767 & -2701 & -10755 & & 0 & -170223 & -108665 \\
\hline 7. & 0 & -165860 & -2890 & -11185 & & 0 & -179935 & -119164 \\
\hline 8. & 0 & -175479 & -3093 & -11633 & & 0 & -190205 & -12944 \\
\hline 9. & 0 & -185657 & -3309 & -12098 & & 0 & -201064 & -13949 \\
\hline 10 & 0 & -196425 & -3541 & -12582 & & 0 & -212548 & -149344 \\
\hline 1 & 0 & -207818 & -3789 & -13085 & & 0 & -224692 & -158981 \\
\hline 2. & 0 & -219871 & -4054 & -13609 & & 0 & -237534 & -168413 \\
\hline 3 & 0 & -232624 & -4338 & -14153 & & 0 & -251115 & -177647 \\
\hline 4. & 0 & -246116 & -4641 & -14719 & & 0 & -265476 & -186685 \\
\hline 5 & 0 & -260391 & -4966 & -15308 & & 0 & -280665 & -195533 \\
\hline 16. & 0 & -275494 & -5314 & -15920 & & 0 & -296728 & -204191 \\
\hline 17. & 0 & -291472 & -5686 & -16557 & & 0 & -313715 & -212673 \\
\hline 18. & 0 & -308378 & -6084 & -17219 & & 0 & -331681 & -220973 \\
\hline 19. & 0 & -326263 & -6510 & -17908 & & 0 & -350681 & -22909 \\
\hline 20. & 0 & -345187 & -6765 & -18625 & & 0 & -370777 & -237054 \\
\hline 21. & 0 & -365208 & -7453 & -19370 & & 0 & -392031 & $-24484 ?$ \\
\hline 22. & 0 & -386390 & -7975 & -20144 & & 0 & -414507 & -252460 \\
\hline 23. & 0 & -408800 & -8533 & -20950 & $=$ & 0 & -438283 & -259931 \\
\hline 24 . & 0 & -432511 & -9130 & -21788 & & 0 & -463429 & -26723 \\
\hline 25. & 0 & -457596 & -9769 & -22660 & & 0 & -490025 & -27439 \\
\hline 20. & 0 & -484137 & -10453 & -23566 & & 0 & -518156 & -28140 \\
\hline 27. & 0 & -512217 & -11185 & -24509 & & 0 & -547911 & -288259 \\
\hline 28. & 0 & -541925 & -11968 & -25489 & & 0 & -579382 & -294975 \\
\hline 29. & 0 & -573357 & -12806 & -26509 & & 0 & -612672 & -301551 \\
\hline 30. & 0 & -606612 & -13702 & -27569 & & 0 & -647383 & -30798 \\
\hline 31. & 0 & -641795 & -14661 & -28672 & & 0 & -685128 & -31429 \\
\hline 32. & 0 & -679019 & -15687 & -29818 & & 0 & -724524 & -320466 \\
\hline 33. & 0 & -718402 & -16786 & -31011 & & 0 & -766199 & -326511 \\
\hline 34. & 0 & -760070 & $=17961$ & -32252 & & 0 & -810283 & -332429 \\
\hline 35. & 0 & -804154 & -19218 & -33542 & & 0 & -856914 & -338225 \\
\hline 36 & 0 & -850795 & -20563 & -34883 & & 0 & -906241 & -343900 \\
\hline 37. & 0 & -900141 & -22003 & -36279 & & 0 & -958423 & -349458 \\
\hline 38. & 0 & -952349 & -23543 & -37730 & & 0 & -1013622 & -35490 \\
\hline 39. & 0 & -1007585 & -25191 & -39239 & & 0 & -1072015 & -36023 \\
\hline 40. & 0 & -1066025 & -26954 & -40809 & & 0 & -1133788 & -365448 \\
\hline 41. & 0 & -1127855 & -28841 & -42441 & & 0 & -1199137 & -37055 \\
\hline 42. & 0 & -1193270 & -30860 & -44139 & & 0 & -1268269 & -375564 \\
\hline 43. & 0 & -1262480 & -33020 & -45904 & 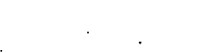 & 0 & -1341404 & -38046 \\
\hline 44. & 0 & -1335704 & -35331 & -47740 & & 0 & -1418775 & -38526 \\
\hline 45. & 0 & -1413175 & -37804 & -49650 & & 0 & -1500629 & -38996 \\
\hline 5. & $0^{\circ}$ & -1495139 & -40451 & -51636 & & 0 & -1587226 & -39457 \\
\hline 7. & 0 & -1581857 & -43282 & -53701 & & 0 & -1678840 & -39908 \\
\hline $48:$ & 0 & -1673605 & -46312 & -55849 & & 0 & -1775766 & -40349 \\
\hline 49. & 0 & -1770674 & -49554 & -58083 & & 0 & -1878311 & -40783 \\
\hline 50. & 0 & -1873373 & -53023 & -60407 & & 0 & -1986803 & -41205 \\
\hline
\end{tabular}

PW $\quad-407000 \quad-3454437 \quad-71636 \quad-187513$ 
NORMANS KILL HYIRO-ELECTRIC FEASIBTLITY STUMY CASH FLOW PROGRAM (CFP1.)

PROJECT A

¿JO YEAR AINALYSIS PERIOI ASSUMING 8.000 PERCENT IIISCOUNT RATE

CAPITAL + LABOR + POWER

\section{$-1309900$}

$1 . \quad 0$

2.

3.

4.

5.

6.

7.

8.

9.

10 .

11 .

12.

13.

14.

15.

16.

17.

18.

19.

20.

21.

22.

23.

24.

25.

26.

27.

28.

29.

30 .

31.

32.

33.

34.

35.

36.

37.

38.

39.

40.

41.

42.

43.

44.

45.

to.

+7 .

+8 .

49.

50 .

PW
$-19709$

$-20853$

$-22062$

$-23342$

- 24696

$-26128$

$-27643$

$-29247$

$-30943$

$-32738$

$-34636$

$-36645$

$-38771$

$-41019$

$-43398$

$-45916$

$-48579$

$-51396$

$-54377$

$-57531$

$-60868$

$-64398$

$-68133$

$-72085$

$-76266$

$-80689$

$-85369$

$-9.0321$

$-75560$

$-101102$

$-106960$

$-113170$

$-119734$

$-126678$

$-134026$

$-141799$.

$-150023$

$-158725$

$-167931$

$-177671$

$-187976$

$-198878$

$-210413$

$-222617$

$-235529$

$-249190$

$-263643$

$-278934$

$-295112$

$-312229$
$-16585$

$-17746$

$-18988$

$-20317$

$-21740$

- 23261

$-24890$

$-26632$

$-28496$

$-30491$

$-32625$

$-34909$

$-37353$

$-39967$

$-42765$

$-45759$

$-48762$

$-52389$

-56056

$-59980$

$-64179$

$-68671$

$-73478$

$-78622$

$-84125$

$-90014$

$-96315$

$-103057$

$-117990$

$-126249$

$-135087$

$-144543$

$-154661$

$-165487$

$-177071$

$-189466$

$-202729$

$-216920$

$-232104$

$-248351$

$-265736$

$-284337$

$-304241$

$-325538$

$-348326$

$-.3727 .08$

$-398798$

$-426714$

$-456584$
$-110271$
$+0 \& M$

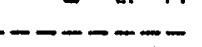

$-47008$

$-48888$

$-50844$

- 52878

$-54993$

$-57192$

$-59480$

$-61859$

$-64334$

$-66907$

$-69583$

$-72367$

$-75261$

$-78272$

$-81403$

$-84659$

$-88045$

$-91567$

$-95230$

$-99039$

$-103000$

$-107120$

$-111405$

- 115861

$-120496$

$-125316$

$-130328$

$-135541$

$-140963$

$-146602$

$-152466$

$-158564$

$-164907$

$-171503$

$-178363$

$-185498$

$-192918$

$-200634$

$-208660$

$-217006$

$-225686$

$-234714$

$-244102$

$-253866$

$-264021$

$-274582$

$-285565$

$-296988$

$-308867$

$-321222$

$-616863$
+ PWR.CR. =

63665

68122

72890

77992

83452

89293

95544

102232

109388

117046

125239

134005

143386

153423

164162

175654

187950

201106

215183

230246

246363

263609

282062

301806

322932

345537

369725

395606

423298

452929

484634

518559

554858

593698

635257

679725

727305

778217

832692

890980

953349

1020083

1091489

1167893

1249646

1337121

1430720

1530870

1638031

1752693

$=$ NET CUM. PW

1309900

$-19637$

$-19365$

$-19004$

$-18545$

$-17977$

$-17288$

$-16469$

$-15506$

$-14385$

$-13090$

$-11605$

$-9916$

$-7999$

$-5835$

$-3404$

$-680$

2364

5754

9520

13696

18310

23420

29046

35238 
HORMANG KILL HYIIRO-ELECTRIC FEASIEILITY STUIY

CASH FLOW PROGRAM (CFP1.)

PROJECT H

50 YEAR GNALYSIS PERIOI ASSUMING 8.000'PERCENT IIISCOUNT RATE

CAPITAL

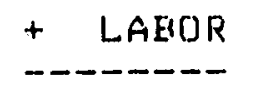

+ POWER + 0 \& i

+ PWR.CR.

$=\quad$ NET

CUM. PW

$0 .-1747200$

1.

$-19709$

$-20853$

$-3745$

$-57200$

$-4007$

$-59488$

$-22062$

$-4288$

$-4588$

$-23342$

$-61868$

4.

$-24696$

$-64342$

$-26128$

$-4909$

$-27643$

$-29247$

$-30943$

$-32738$

$-34636$

-36645

$-38771$

$-41019$

$-43398$

$-45916$

$-48579$

$-51376$

$-54377$

$-57531$

$-60868$

$-64398$

$-68133$

$-72085$

$-70266$

$-80689$

$-85369$

$-90321$

$-95560$

$-101102$

$-106766$

$-113170$

$-119734$

$-126678$

$-134026$

$-141799$

$-150023$

$-158725$

$-167931$

$-177671$

- 187976

$-198878$

$-210413$

- 222617

$-235529$

$-249190$

$-263643$

$-278934$

-295112

$-312229$

$-5253$

$-66916$

$-69593$

$-72376$

$-6014$

$-6435$

$-6885$

$-7367$

$-7883$

$-8434$

$-9025$

$-9657$

$-10333$

$-11056$

$-11830$

$-75271$

$-78282$

$-81413$

$-84670$

$-88057$

$-91579$

-95242

$-99052$

$-103014$

$-107135$

$-12658$

$-111420$

$-13544$

$-14492$

$-15506$

- 16592

$-17753$

- 18996

$-20326$

$-21749$

$-23271$

$-24900$

$-26643$

$-28508$

$-30503$

$-32639$

$-34923$

$-37368$

$-39984$

$-42783$

$-45777$

- 48982

$-52411$

$-56079$

$-60005$

$-64205$

$-68700$

$-73509$

$-78654$

$-84160$

$-90051$

$-96355$

$-115877$

$-120512$

$-125332$

$-130346$

$-135559$

$-140982$

$-146621$

$-152486$

$-158585$

$-164929$

$-171526$

$-178387$

$-185522$

$-192943$

$-200601$

- 208687

$-217035$

$-225716$

$-234745$

$-244135$

$-253900$

$-264056$

$-274618$

$-285603$

$-297027$

$-308908$

$-321265$

$-334115$

- 347480

-361379

$-375834$

$-390868$

76826
82204
87958

94115

100703

107752

115295

123366

132001

141241

151128

161707

173027

185139

198098

211965

226803

242679

259667

277843

297292

318103

340370

364196

389690

416968

446156

477387

510804

546560

584819

625756

669559

716429

766579

820239

877656

939092

1004828

1075166

1150428

1230958

1317125

1409323

1507976

1613534

1726482

1847335

1976649

2115014
$-1747200$

$-3828$

$-2144$

$-260$

- 1843

4182

6778

9656

12834

16341

20205

24455

29122

34243

39853

45991

52702

60033

68033

76755

86256

96600

107853

120086

133376

147807

163467

180453

198866

218818

240428

263823

289140

316525

346141

378150

412740

450105

490455

534015.

581028

631755

686472

745480

809098

877673

951575

1031199

1116971

1209348

1308817

$-1747200$

$-1750744$

$-1752583$

$-1752789$

$-1751434$

$-1748588$

$-1744317$

$-1738683$

$-1731749$

$-1723574$

$-1714215$

$-1703727$

$-1692162$

$-1679571$

- 1606003

$-1651505$

- 1636121

-1617896

$-1602871$

$-1585086$

$-1566580$

$-1547390$

$-1527551$

$-1507099$

$-1486060$

$-1464483$

$-1442382$

$-1419792$

$-1396740$

$-1373255$

$-1349362$

$-1325086$ 
NORMANS KTLL HYIIRO-ELECTRTC FEASIEILTTY STUIY IIELTA CASH FLOW PROGRAP ( $\triangle C F P 1$ )

PROJECT A LESS BASELINE

SO YEAR ANALYSIS PERIOI ASSUHING 8.000 PERCENT IIISCOUNT RATE

'R.

CAPITAL

+ LABOR

+ POWER

+0 is i

+ PWR,CR

$-902900$

1.

2.

3.

4.

5.

6.

7.

8.

9.

10.

11.

12.

13.

14 .

15.

1.6 .

17.

18.

19.

20 .

21.

22.

23.

24.

25.

26.

27.

28.

29.

30.

31 .

32.

33.

34 .

35.

36.

37.

33.

39.

40.

41.

42.

43.

44

45

10.

$+7$.

$+8$.

49.

50 .
98548

104263

110311

116708

1. 23477

130639

138217

146232

154714

163687

1.73182

183226

193853

205097

216.993

229578

242893

256982

271836

287656

304340

321992

340667

360426

381330

403448

426848

451604

477797

505510

534829

565849

598668

633392

670128

708996

750118

793624

839654

883354

994392

1052067

1113087

1177646

1245949

1319214

1394671

1475562

1561144
939879

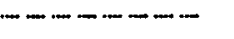

$-14659$

$-15685$

$-16783$

$-17953$

$-19215$

$-20560$

$-22000$

$-23539$

$-25187$

$-26950$

$-28836$

$-30855$

$-33015$

$-35326$

$-37799$

$-40445$

$-43276$

$-46305$

$-49546$

$-53015$

$-56726$

$-60696$

$-64945$

$-69492$

$-74356$

$-79561$

$-85130$

$-91089$

$-97465$

$-104288$

$-111588$

$-119400$

$-127757$

$-136700$

$-146269$

$-156508$

$-167463$

$-179186$

$-191729$

$-205150$

$-219510$

$-234876$

$-251317$

$-268910$

$-287734$

307875

$-329426$

-352486

$-3771.60$

$-403561$
38168

$-39694$

$-4.283$

$-1+2934$

$-4.4651$

$-46437$

$-48295$

$-50226$

$-52236$

$-54325$

$-56498$

$-58758$

$-61108$

$-63553$

$-66095$

$-68739$

$-71488$

$-74348$

$-77322$

$-80414$

$-83630$

$-86976$

$-904.55$

$-94073$

$-97836$

$-101.750$

$-105819$

$-110052$

$-114454$

$-119033$

$-123794$

$-128746$

$-133896$

$-139251$

$-144821$

$-150615$

$-156639$

$-162904$

$-169421$

$-176197$

$-183245$

$-198198$

$-206126$

$-214371$

$-222946$

$-231.864$

$-241139$

$-250784$

$-260815$
$-190575$
NET

$-$

$-902900$

109336

117006

1.25135

133800

143063

152935

163466

174699

186679

1.99458

213087

227618

243116

259641

277261

296048

316079

337435

360201.

384473

410347

437929

467329

498667

532070

567674 605624

646069

689176

735118

784081 .

836262

891873

951139

1014295

1081598

1153321

1229751

1311196

1397987

1490473

1539024

1694041

1805944

1925187

2052249

213764.4

2331916

2435649

2649461

cuit, PW

$-902900$

$-801617$

$-701303$

$-601967$

$-503614$

$-406248$

$-309873$

$-214492$

$-120107$ $-26721$ 65666 157056 24.7446 336839 425237

512641

599055

684481 
NORMANG KILL HYIRO-ELECTRIC FEASTEILITY STUIY IUELTA CASH FLOW PROGRAM (ACFPI)

PROJECT H LESS EIASELINE

50 YEAR ANALYSIS PERIOI ASSUMING 8.000 PERCENT IITSCDUNT RATE

\begin{tabular}{|c|c|c|c|c|c|c|}
\hline CAPITAL & $+\quad \angle A F O R$ & $+\quad$ POWER & $D \& M$ & $+P W R, C R$. & NET. & CUM. PW \\
\hline$--\infty-\infty--$ & $\cdots---\cdots$ & -------- & - & $-----\cdots$ & $-\cdots-\cdots-\cdots$ & ------- \\
\hline 340200 & & & & & -1340200 & -1340200 \\
\hline 0 & 98548 & -1819 & -48360 & 76826 & 125195 & -1224279 \\
\hline 0 & 104263 & -1946 & -50294 & 82204 & 134227 & -1109201 \\
\hline 0 & 110311 & -2083 & -52307 & 87958 & 143879 & -994985 \\
\hline 0 & 116708 & - 2229 & -54398 & 94115 & 154196 & -881646 \\
\hline 0 & 123477 & -2384 & -56574 & 100703 & 165222 & -769195 \\
\hline 0 & 130639 & -2552 & -58838 & 107752 & 177001 & -657658 \\
\hline 0 & 138217 & -2730 & -61191 & 115295 & 189591 & -547034 \\
\hline 0 & 146232 & -2921 & -63638 & 123366 & 203039 & -43733 \\
\hline 0 & 154714 & -3126 & -66184 & 132001 & 217405 & -328581 \\
\hline 0 & 163687 & -3344 & -68831 & 141241 & 232753 & -220772 \\
\hline 0 & 173182 & -3578 & -71585 & 151128 & 249147 & -11391 \\
\hline 0 & 183226 & -3829 & -74448 & 161707 & 266656 & -802 \\
\hline 0 & 193853 & -4096 & -77426 & 173027 & 285358 & 9690 \\
\hline 0 & 205097 & -4384 & -80523 & 185139 & 305329 & 20085 \\
\hline 0 & 216993 & -4691 & -83744 & 198098 & 326656 & 30383 \\
\hline 0 & 229578 & -5019 & -87094 & 211965 & 349430 & 40582 \\
\hline 0 . & 242893 & -5370 & -90578 & 226803 & 373748 & 50683 \\
\hline 0 & 256982 & -5746 & -94201 & 242679 & 399714 & 60686 \\
\hline 0 & 271886 & -6148 & -97969 & 259667 & 427436 & 70590 \\
\hline 0 & 287650 & -6579 & -101887 & 277843 & 457033 & 80396 \\
\hline 0 & 304340 & -7039 & -105962 & 297292 & 488631 & 90103 \\
\hline 0 & 321992 & -7531 & -110202 & 318103 & 522362 & 99711 \\
\hline 0 & 340667 & -8059 & -114609 & 340370 & 558369 & 109221 \\
\hline 0 & 360426 & -8623 & -119194 & 364196 & 596805 & 118633 \\
\hline 0 & 381330 & -9227 & -123961 & 389690 & 637832 & 127946 \\
\hline 0 & 403448 & -9873 & -128920 & 416968 & 681623 & 137162 \\
\hline 0 & 426848 & -10504 & -134076 & 446156 & 728364 & 146280 \\
\hline 0 & 451604 & -11303 & -139440 & 47.7387 & 778248 & 155301 \\
\hline 0 & 477797 & -12094 & -145017 & 510804 & 831490 & 164225 \\
\hline 0 & 505510 & -12941 & -150818 & 546560 & 888311 & 173053 \\
\hline 0 & 534829 & -13847 & -156850 & 584819 & 948951 & 181785 \\
\hline 0 & 565849 & -14816 & -163125 & 625756 & 1013664 & 190421 \\
\hline 0 & 598668 & -15853 & -169650 & 669559 & 1082724 & 198963 \\
\hline 0 & 633392 & -16962 & -176435 & 716429 & 1156424 & 207410 \\
\hline 0 & 670128 & -18150 & -183493 & 766579 & 1235064 & 215763 \\
\hline 0 & 708996 & -19421 & -190833 & 820239 & 1318981 & 224023 \\
\hline 0 & 750118 & -20780 & $-198466^{\circ}$ & 877656 & 1408528 & 232191 \\
\hline 0 & 793624 & -22234 & -206405 & 939092 & 1504077 & 240266 \\
\hline 0 & 839654 & -23791 & 214661 & 1004828 & 1606030 & 248250 \\
\hline 0 & 888354 & -25457 & 223247 & 1075166 & 1714816 & 250144 \\
\hline 0 & 939879 & -27238 & -232177 & 1150428 & 1830892 & 263947 \\
\hline 0 & 994392 & -29145 & 241464 & 1230958 & 1954741 & 271661 \\
\hline 0 & 1052067 & -31185 & -251123 & 1317125 & 2086884 & 27928 \\
\hline 0 & 1113087 & -33369 & -261168 & 1409323 & 2227873 & 286825 \\
\hline 0 & 1177646 & -35705 & -271615 & 1507976 & 2378302 & 294276 \\
\hline 0 & 1245949 & -38203 & -282479 & 1613534 & 2538801 & 301640 \\
\hline 0 & 1318214 & -40878 & -293779 & 1726482 & 2710039 & 308915 \\
\hline 0 & 1394671 & -43739 & -305530 & 1847335 & 2892737 & 31611 \\
\hline 0 & 1475562 & -46801 & -317751 & 1976649 & 3087659 & $32322=$ \\
\hline 0 & 1561144 & -50077 & -330461 & 2115014 & 3295620 & 33024 \\
\hline
\end{tabular}

Pw $\begin{array}{llllll}-1340200 & 2878698 & -67657 & -1025809 & 2857465 & 3302497\end{array}$ 


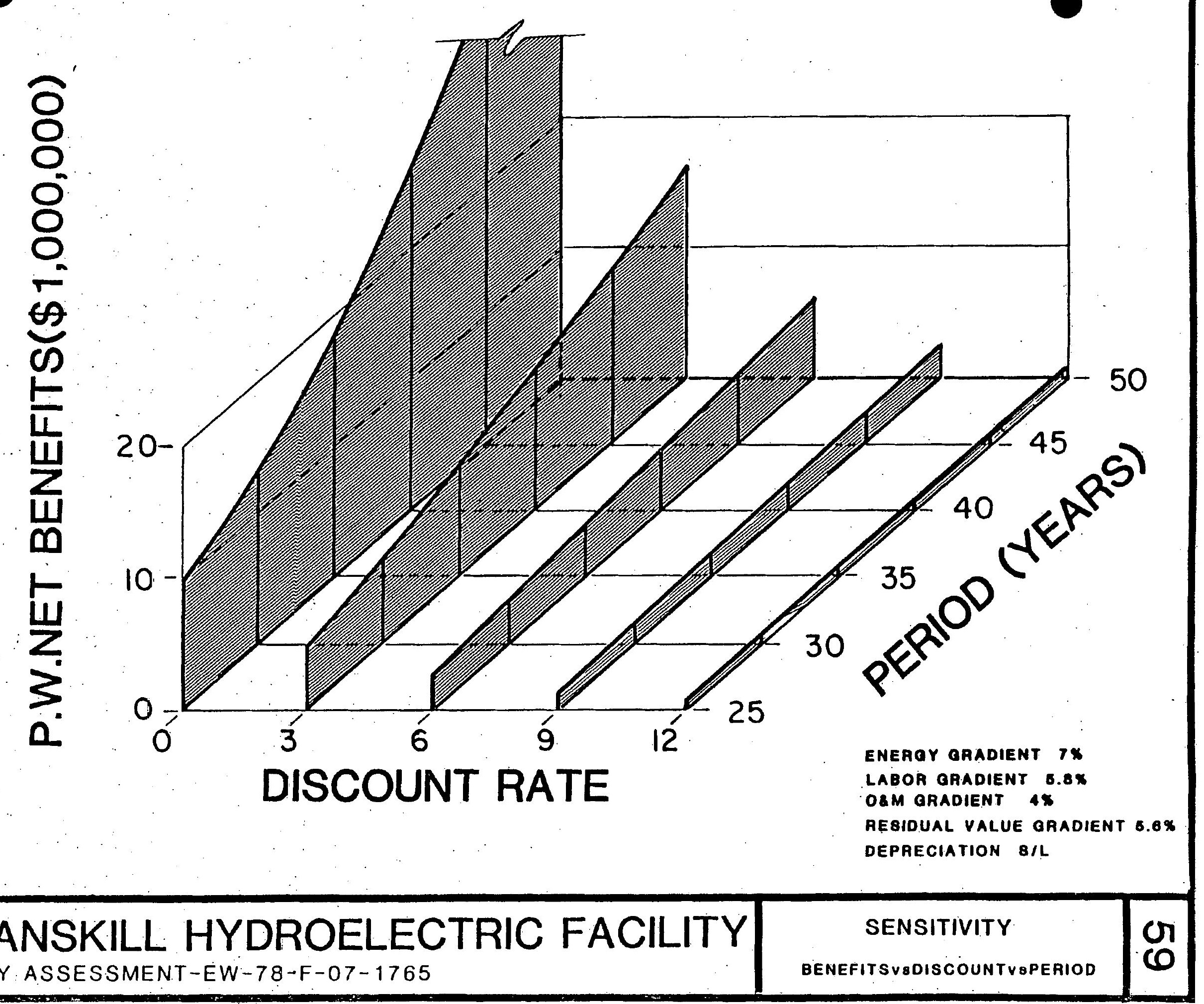




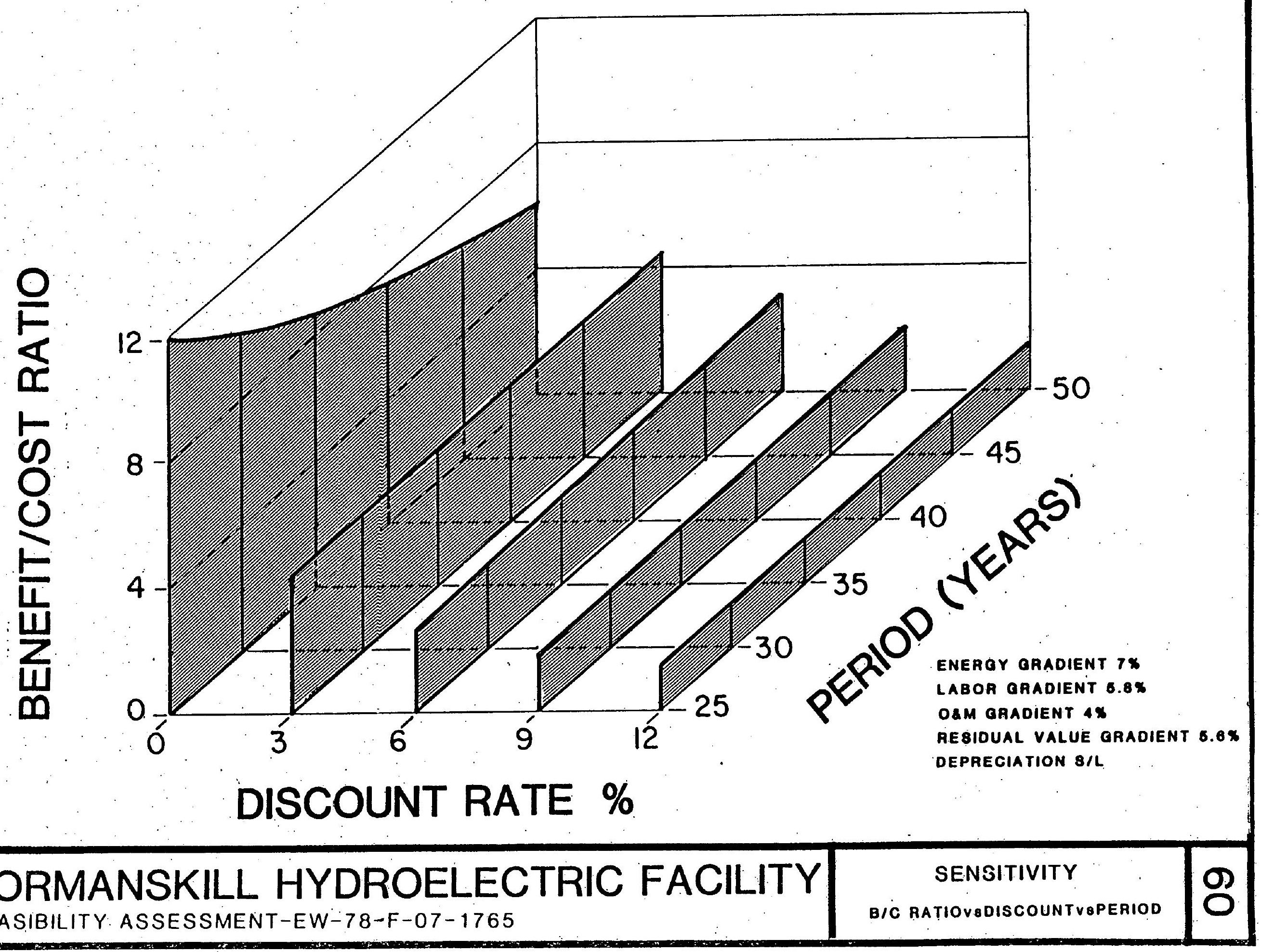




\section{DATA MATRIX FOR FIGURES $59 \& 60$}

\begin{tabular}{|c|c|c|c|c|c|c|}
\hline $\begin{array}{l}\text { APAALYSIS } \\
\text { PERIOI } \\
\text { (YEARS) }\end{array}$ & $\begin{array}{c}\text { IISCOUNT } \\
\text { RATE } \\
\text { (\%/YEAR) }\end{array}$ & $\begin{array}{c}\text { LAEIOR } \\
\text { GRAIIIENT } \\
\text { (\%/YEAR) }\end{array}$ & $\begin{array}{l}\text { ENERGY } \\
\text { GRAIIIENT } \\
\text { ( } \% / \text { Y YEAR). }\end{array}$ & $\begin{array}{c}\text { CAPITAL } \\
\text { COST } \\
\text { FACTOR }\end{array}$ & $\begin{array}{l}\text { P.W. NET } \\
\text { BENEFITS } \\
(\$ / 1000)\end{array}$ & $\begin{array}{c}\text { BEREFIT } \\
\text { TO COST } \\
\text { RATIO }\end{array}$ \\
\hline - - - - - & ------- & -ーーーーーー & - - - - - - & $--\cdots---$ & $---\cdots--$ & -ーーーーーー \\
\hline $\begin{array}{l}25 \\
25 \\
25 \\
25 \\
25\end{array}$ & $\begin{array}{r}.00 \\
3.00 \\
6.00 \\
9.00 \\
12.00\end{array}$ & $\begin{array}{l}5.80 \\
5.80 \\
5.80 \\
5.80 \\
5.80\end{array}$ & $\begin{array}{l}7.00 \\
7.00 \\
7.00 \\
7.00 \\
7.00\end{array}$ & $\begin{array}{lll}1 . & 0 & 0 \\
1 . & 0 & 0 \\
1 . & 0 & 0 \\
1 . & 0 & 0 \\
1 . & 0 & 0\end{array}$ & $\begin{array}{r}9263 \\
4936 \\
2610 \\
1302 \\
532\end{array}$ & $\begin{array}{r}11.86 \\
4.33 \\
2.54 \\
1.75 \\
1.31\end{array}$ \\
\hline $\begin{array}{l}30 \\
30 \\
30 \\
30 \\
30\end{array}$ & $\begin{array}{r}.00 \\
3.00 \\
6.00 \\
9.00 \\
12.00\end{array}$ & $\begin{array}{l}5.80 \\
5.80 \\
5.80 \\
5.80 \\
5.80\end{array}$ & $\begin{array}{l}7.00 \\
7.00 \\
7.00 \\
7.00 \\
7.00\end{array}$ & $\begin{array}{lll}1.0 & 0 \\
1.0 & 0 \\
1.0 & 0 \\
1.0 & 0 \\
1.0 & 0\end{array}$ & $\begin{array}{r}13304 \\
6522 \\
3240 \\
1555 \\
633\end{array}$ & $\begin{array}{r}10.02 \\
4.38 \\
2.64 \\
1.82 \\
1.35\end{array}$ \\
\hline $\begin{array}{l}35 \\
35 \\
35 \\
35 \\
35\end{array}$ & $\begin{array}{r}.00 \\
3.00 \\
6.00 \\
9.00 \\
12.00\end{array}$ & $\begin{array}{l}5.80 \\
5.80 \\
5.80 \\
5.80 \\
5.80\end{array}$ & $\begin{array}{l}7.00 \\
7.00 \\
7.00 \\
7.00 \\
7.00\end{array}$ & $\begin{array}{l}1.00 \\
1.00 \\
1.00 \\
1.00 \\
1.00\end{array}$ & $\begin{array}{r}18699 \\
8395 \\
3905 \\
1795 \\
721\end{array}$ & $\begin{array}{l}8.64 \\
4.43 \\
2.75 \\
1.88 \\
1.38\end{array}$ \\
\hline $\begin{array}{l}40 \\
40 \\
40 \\
40 \\
40\end{array}$ & $\begin{array}{r}.00 \\
3.00 \\
6.00 \\
9.00 \\
12.00\end{array}$ & $\begin{array}{l}5.80 \\
5.80 \\
5.80 \\
5.80 \\
5.80\end{array}$ & $\begin{array}{l}7.00 \\
7.00 \\
7.00 \\
7.00 \\
7.00\end{array}$ & $\begin{array}{lll}1.0 & 0 \\
1.0 & 0 \\
1.0 & 0 \\
1.0 & 0 \\
1.0 & 0\end{array}$ & $\begin{array}{r}25914 \\
10608 \\
4605 \\
2023 \\
797\end{array}$ & $\begin{array}{l}7.60 \\
4.47 \\
2.86 \\
1.95 \\
1.42\end{array}$ \\
\hline $\begin{array}{l}45 \\
45 \\
45 \\
45 \\
45\end{array}$ & $\begin{array}{r}.00 \\
3.00 \\
6.00 \\
9.00 \\
12.00\end{array}$ & $\begin{array}{r}5.80 \\
5.80 \\
5.80 \\
5.80 \\
5.80\end{array}$ & $\begin{array}{l}7.00 \\
7.00 \\
7.00 \\
7.00 \\
7.00\end{array}$ & $\begin{array}{lll}1.0 & 0 \\
1.0 & 0 \\
1.0 & 0 \\
1.0 & 0 \\
1.0 & 0\end{array}$ & $\begin{array}{r}35579 \\
13224 \\
5340 \\
2236 \\
861\end{array}$ & $\begin{array}{r}0.78 \\
4.51 \\
2.97 \\
2.01 \\
1.44\end{array}$ \\
\hline $\begin{array}{l}50 \\
50 \\
50 \\
50 \\
50\end{array}$ & $\begin{array}{r}.00 \\
3.00 \\
6.00 \\
9.00 \\
12.00\end{array}$ & $\begin{array}{l}5.80 \\
5.80 \\
5.80 \\
5.80 \\
5.80\end{array}$ & $\begin{array}{l}7.00 \\
7.00 \\
7.00 \\
7.00 \\
7.00\end{array}$ & $\begin{array}{lll}1.0 & 0 \\
1.0 & 0 \\
1.0 & 0 \\
1.0 & 0 \\
1.0 & 0\end{array}$ & $\begin{array}{r}48548 \\
16317 \\
6109 \\
2435 \\
915\end{array}$ & $\begin{array}{l}6.13 \\
4.54 \\
3.08 \\
2.07 \\
1.4 .7\end{array}$ \\
\hline
\end{tabular}





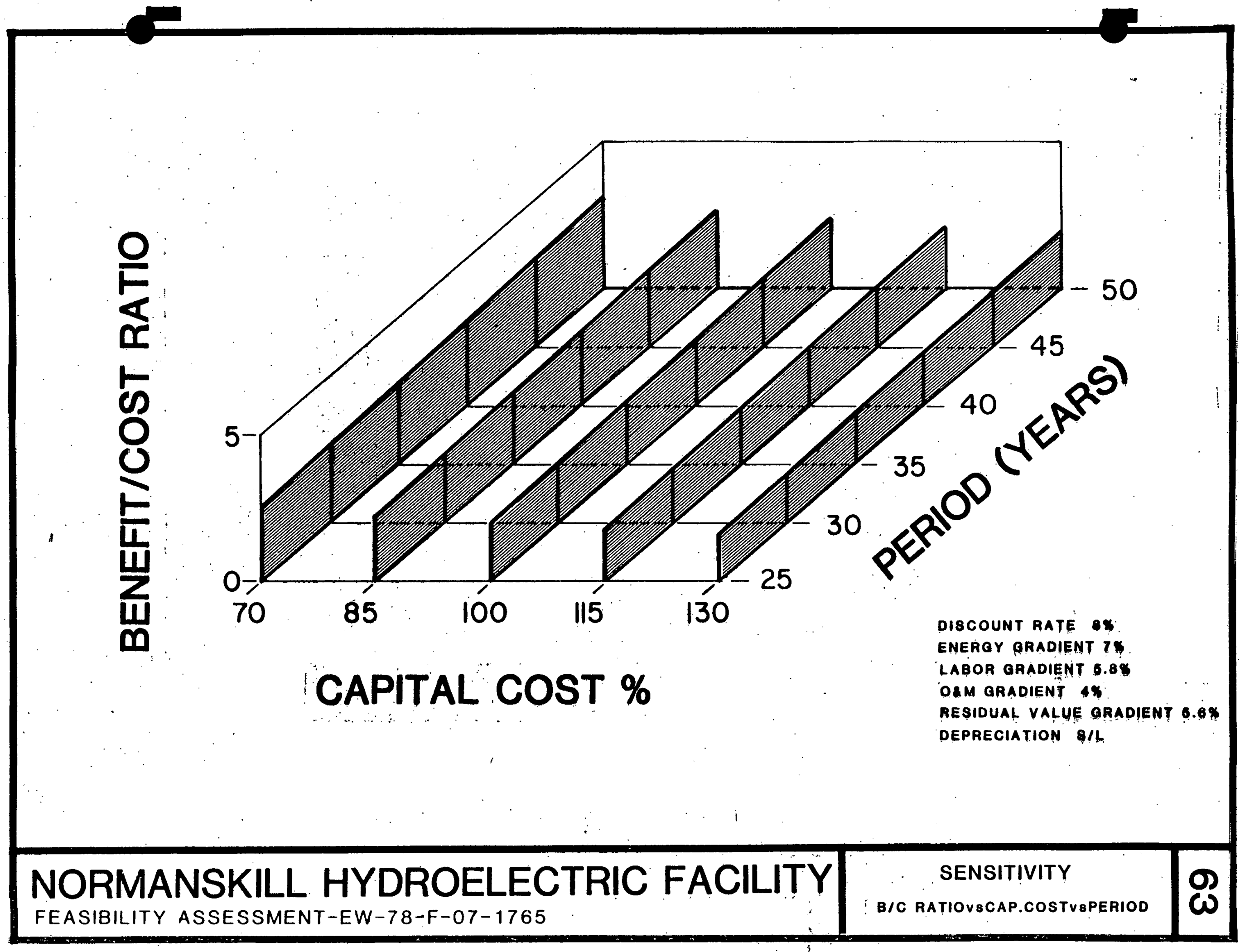




\section{DATA MATRIX FOR FIGURES $62 \& 63$}

\begin{tabular}{|c|c|c|c|c|c|c|}
\hline $\begin{array}{l}\text { ANALYSIS } \\
\text { PERIOII. } \\
\text { (YEARS) }\end{array}$ & $\begin{array}{l}\text { IISCOUNT } \\
\text { RATE } \\
(\% / Y E A R)\end{array}$ & $\begin{array}{c}\text { LAEQR } \\
\text { GRAIIIENT } \\
(\% / Y E A R)\end{array}$ & $\begin{array}{l}\text { ENERGY } \\
\text { GRAIIENT } \\
\text { (\%/YEAR) }\end{array}$ & $\begin{array}{c}\text { CAPITAL } \\
\text { COST } \\
\text { FACTOR }\end{array}$ & $\begin{array}{l}P . W, \text { NET } \\
\text { EENEFITS } \\
(\$ / 1000)\end{array}$ & $\begin{array}{l}\text { BENEFIT } \\
\text { TO COST } \\
\text { RATIO }\end{array}$ \\
\hline-------- & ------ & $-------m$ & $----\cdots-\cdots$ & ------ & ------- & $------\cdots$ \\
\hline $\begin{array}{l}25 \\
25 \\
25 \\
25 \\
25\end{array}$ & $\begin{array}{l}8.00 \\
8.00 \\
8.00 \\
8.00 \\
8.00\end{array}$ & $\begin{array}{r}5.80 \\
5.80 \\
5.80 \\
5.80 \\
5.80\end{array}$ & $\begin{array}{l}7.00 \\
7.00 \\
7.00 \\
7.00 \\
7.00\end{array}$ & $\begin{array}{r}.70 \\
.85 \\
1.00 \\
1.15 \\
1.30\end{array}$ & $\begin{array}{l}2036 \\
1849 \\
1662 \\
1474 \\
1287\end{array}$ & $\begin{array}{l}2.50 \\
2.20 \\
1.90 \\
1.77 \\
1.61\end{array}$ \\
\hline $\begin{array}{l}30 \\
30 \\
30 \\
30 \\
30\end{array}$ & $\begin{array}{l}8.00 \\
8.00 \\
8.00 \\
8.00 \\
8.00\end{array}$ & $\begin{array}{l}5.80 \\
5.80 \\
5.80 \\
5.80 \\
5.80\end{array}$ & $\begin{array}{l}7.00 \\
7.00 \\
7.00 \\
7.00 \\
7.00\end{array}$ & $\begin{array}{r}.70 \\
.85 \\
1.00 \\
1.15 \\
1.30\end{array}$ & $\begin{array}{l}2421 \\
2212 \\
2004 \\
1795 \\
1586\end{array}$ & $\begin{array}{l}2.60 \\
2.28 \\
2.04 \\
1.84 \\
1.68\end{array}$ \\
\hline $\begin{array}{l}35 \\
35 \\
35 \\
35 \\
35\end{array}$ & $\begin{array}{l}8.00 \\
8.00 \\
8.00 \\
8.00 \\
8.00\end{array}$ & $\begin{array}{l}5.80 \\
5.80 \\
5.80 \\
5.80 \\
5.80\end{array}$ & $\begin{array}{l}7.00 \\
7.00 \\
7.00 \\
7.00 \\
7.00\end{array}$ & $\begin{array}{r}.70 \\
.85 \\
1.00 \\
1.15 \\
1.30\end{array}$ & $\begin{array}{l}2793 \\
2567 \\
2341 \\
2114 \\
1888\end{array}$ & $\begin{array}{l}2.70 \\
2.37 \\
2.12 \\
1.91 \\
1.74\end{array}$ \\
\hline $\begin{array}{l}40 \\
40 \\
40 \\
40 \\
40\end{array}$ & $\begin{array}{l}8.00 \\
8.00 \\
8.00 \\
8.00 \\
8.00\end{array}$ & $\begin{array}{l}5.80 \\
5.80 \\
5.80 \\
5.80 \\
5.80\end{array}$ & $\begin{array}{l}7.00 \\
7.00 \\
7.00 \\
7.00 \\
7.00\end{array}$ & $\begin{array}{r}.70 \\
.85 \\
1.00 \\
1.15 \\
1.30\end{array}$ & $\begin{array}{l}3152 \\
2911 \\
2671 \\
2430 \\
2189\end{array}$ & $\begin{array}{l}2.80 \\
2.46 \\
2.20 \\
1.98 \\
1.81\end{array}$ \\
\hline $\begin{array}{l}45 \\
45 \\
45 \\
45 \\
45\end{array}$ & $\begin{array}{l}8.00 \\
8.00 \\
8.00 \\
8.00 \\
8.00\end{array}$ & $\begin{array}{l}5.80 \\
5.80 \\
5.80 \\
5.80 \\
5.80\end{array}$ & $\begin{array}{l}7.00 \\
7.00 \\
7.00 \\
7.00 \\
7.00\end{array}$ & $\begin{array}{r}.70 \\
.85 \\
1.00 \\
1.15 \\
1.30\end{array}$ & $\begin{array}{l}3497 \\
3244 \\
2992 \\
2739 \\
2486\end{array}$ & $\begin{array}{l}2.90 \\
2.55 \\
2.28 \\
2.06 \\
1.87\end{array}$ \\
\hline $\begin{array}{l}50 \\
50 \\
50 \\
50 \\
50\end{array}$ & $\begin{array}{l}8.00 \\
8.00 \\
8.00 \\
8.00 \\
8.00\end{array}$ & $\begin{array}{l}5.80 \\
5.80 \\
5.80 \\
5.80 \\
5.80\end{array}$ & $\begin{array}{l}7.00 \\
7.00 \\
7.00 \\
7.00 \\
7.00\end{array}$ & $\begin{array}{r}.70 \\
.85 \\
1.00 \\
1.15 \\
1.30\end{array}$ & $\begin{array}{l}3827 \\
3565 \\
3302 \\
3040 \\
2778\end{array}$ & $\begin{array}{l}3.00 \\
2.64 \\
2.36 \\
2.13 \\
1.94\end{array}$ \\
\hline
\end{tabular}




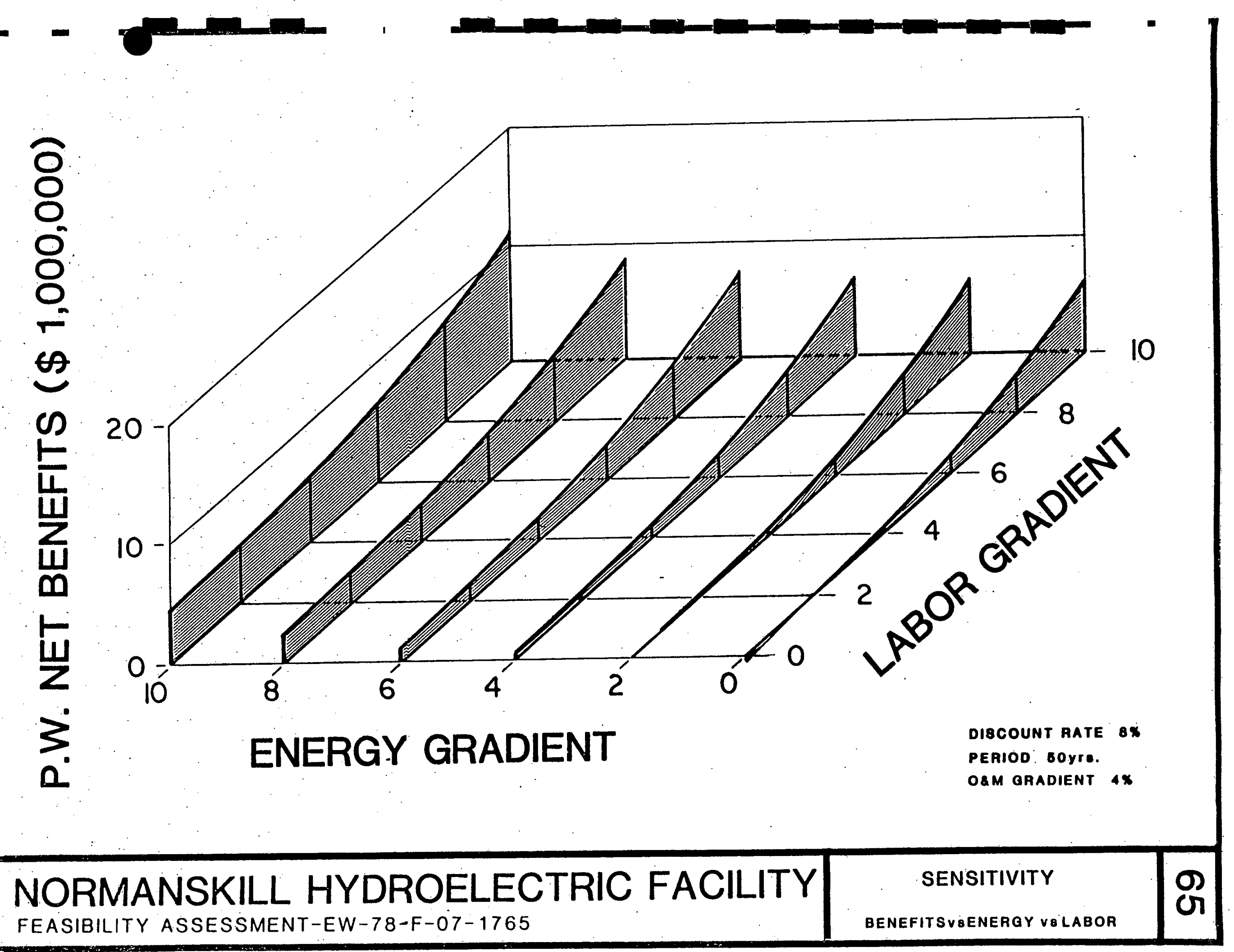




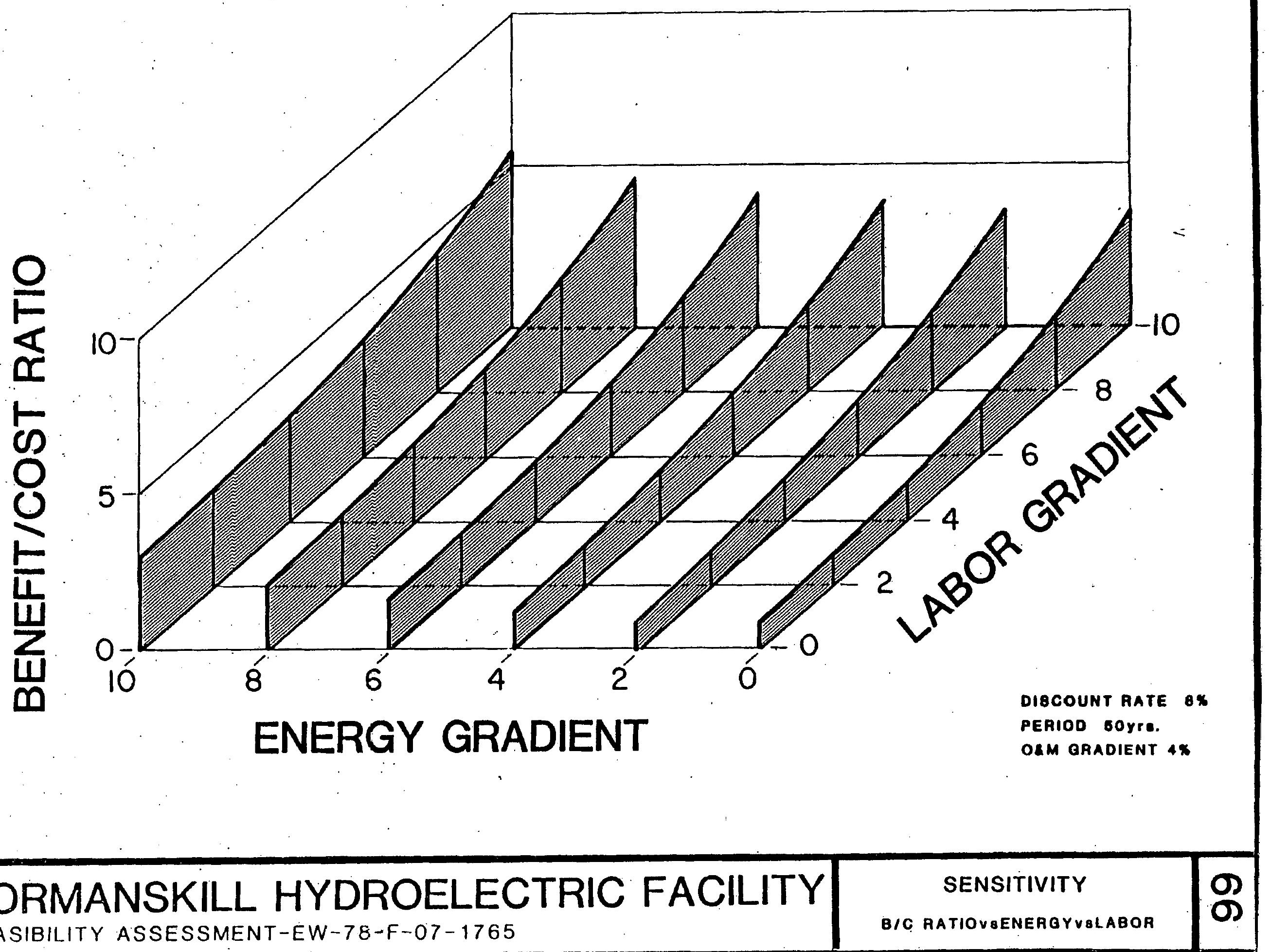




\section{DATA MATRIX FOR FIGURES $65 \& 66$}

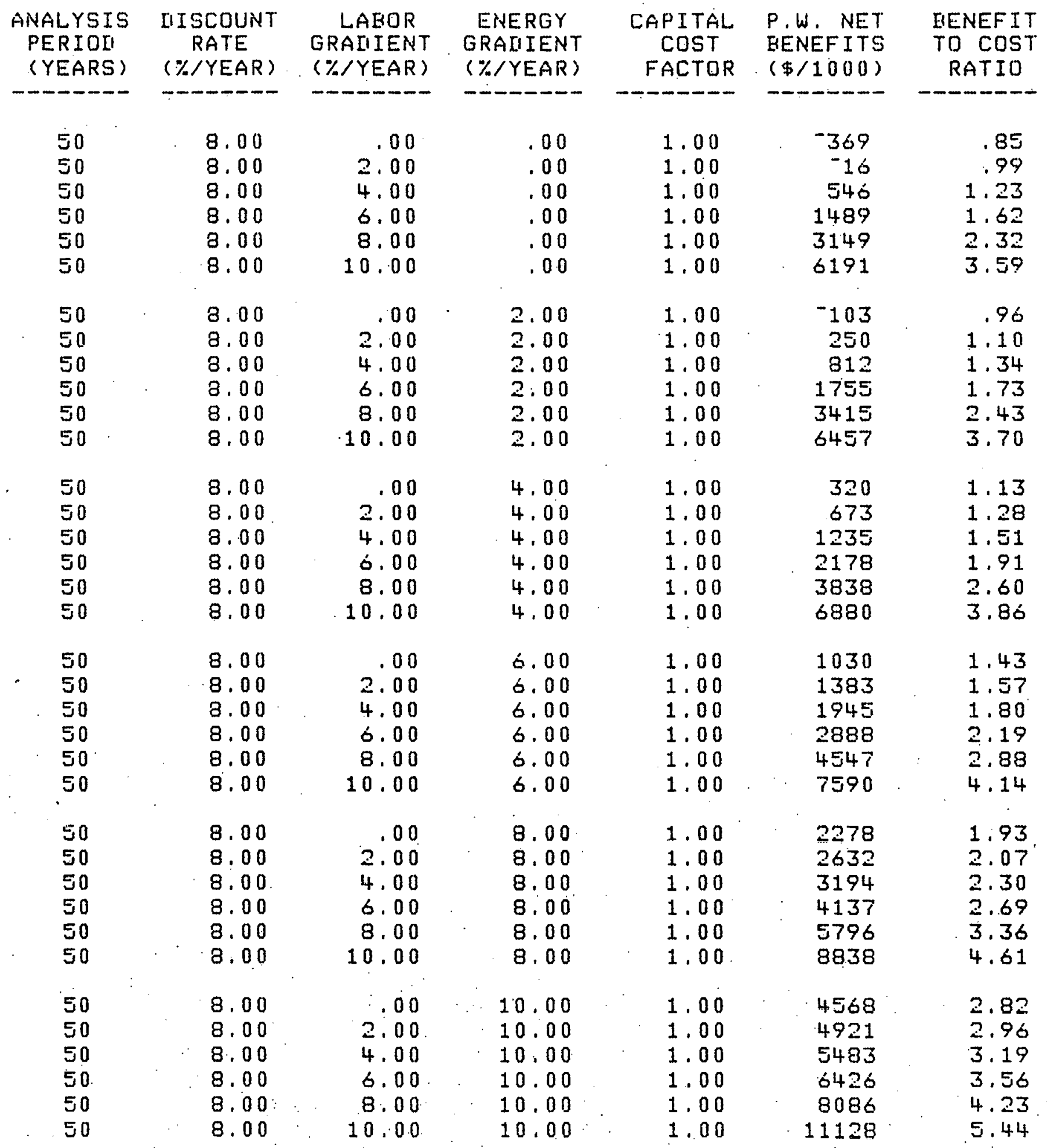




\section{IMPLEMENTATION PLAN}

It has been determined as a matter of policy by the site owner, the City of Watervliet, New York, that a decision on pursuing the Normanskil. Hydroelectric Facility project will be made only after several public hearings held for the benefit of the city taxpayers. A referendum for such a project will not be required. It is expected that such public hearings will involve discussion of two configuration alternatives. One would be the alternative juaged the optimum most feasible resulting in maximum benefits over the life of the project. The second would be a "no project" alternative representing the maintenance of the existing facility alternative with its attendant ramifications for increased energy costs to the city. Acting under municipal law statutes of the state of New York, the only legal requirements for implementation of a selected project would be a majority vote of the two City Councilmen and the Mayor.

\section{PERT}

A PERT (Program Evaluation Review Technique) preliminary activity chart has been prepared and is shown as Figure 69. This technique will be used throughout the design, licensing and construction of the proposed Normanskill Hydroelectric Facility as a means of identifying and eliminating undue time delay. The PERT program to be used will operate on the estimated time completion basis using the standard distribution [Ref. 33] calculated from several estimates varying from most optimistic to most pessimistic. The figure illustrated here is diagrammatic in nature and is not intended to represent all activities and nodes. It is anticipated that during the process of design and licensing construction, the more specific activities will be identified and logged. As can be seen from an examination of Figure 69, the most identifiable and least accurate estimate of time delay is in the licensing process. The decision has been made in the preliminary PERT analysis for application of a Federal Energy Regulatory Commission permit prior 
to license as permitted under the present draft regulations [Ref. 83]. It is hoped that through this process, any initial conceptual problems with the license application will be identified at an early stage and will serve to expedite the formal license review. As shown on Figure 69, the earliest (most optimistic) power on-Iine date is March 1982. It is emphasized that this is based upon the preliminary estimate only. (Note: Immediately prior to issuance of this report, Executive Order 12129 was issued. See Chapter 7 of this report for particulars).

\section{Financing}

Various vehicles were investigated to determine their desirability with regard to the financing of the construction phase of the hydroelectric project. Municipal bonding, Industrial Development Authority bonding, Environmental Facility Corporation bonding, and private financing (lease) were reviewed for this purpose. Municipal bonding appears to be the most attractive avenue for consideration. This form of bonding could either be through the General or Water Fund. Each requires the same course of action. The City Council would pass a resolution citing the reasons for borrowing and the amount sought. Pending approval of a commercial bank, bond anticipation notes would be issued to the city. For the first year only interest would have to be paid on these notes. If desired, the notes could be extended and renewed for an additional two years. As the bond market dictates, a bond sale would be held, for the 1ssuance of municipal bonds. Water fund bonding has no effect upon. the general borrowing power of the city, while general bonding does. The water bonds could extend for a period of 40 years, while general bonds usually hold a 20-year maturity.

City and County Industrial Development Authority (I.D.A.) bonding were eliminated from consideration due to the fact that for a project of this type, they could not legally be a bonding agent without action by the New York state Legislature to modify the I.D.A.'s charters. 
Similarly, The N.Y.S. Environmental Facilities Corporation was. found to be unavailable for financing purposes. This public benefit corporation is commissioned to grant funding for sewer projects only and cannot offer their services for this type of project.

Private financing was discussed with Niagara-Mohawk Power Corporation and, as mentioned in the Marketing section; Niagara-Mohawk Power Corporation would rather purchase the facility rather than lease it. The Power Authority of the state of New York (PASNY) also showed no interest in leasing the facility.

Whether the city finances the project through municipal bonding or federal loans are granted, the decisions and legal responsibility will be that of the city Council. 


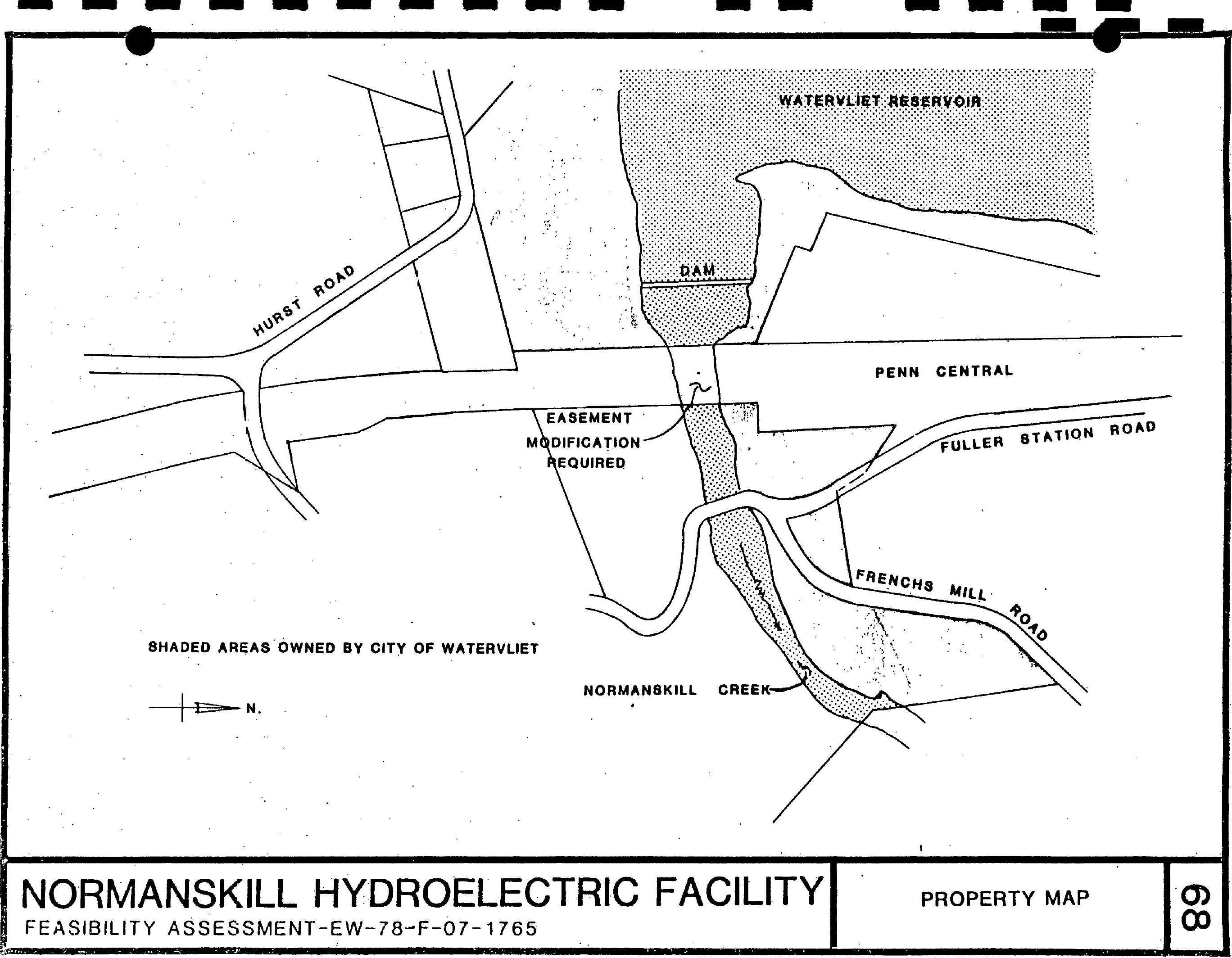




\section{START}

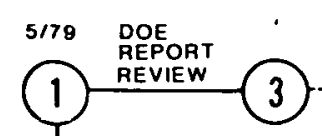

$9 / 79$

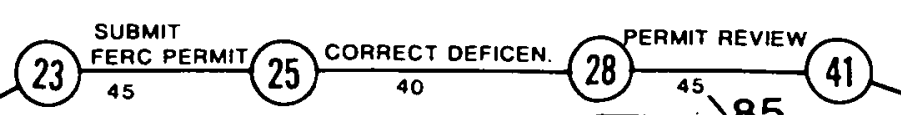

DESIGN \& LICENSE

(15) 20 (20) (21)

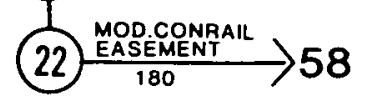

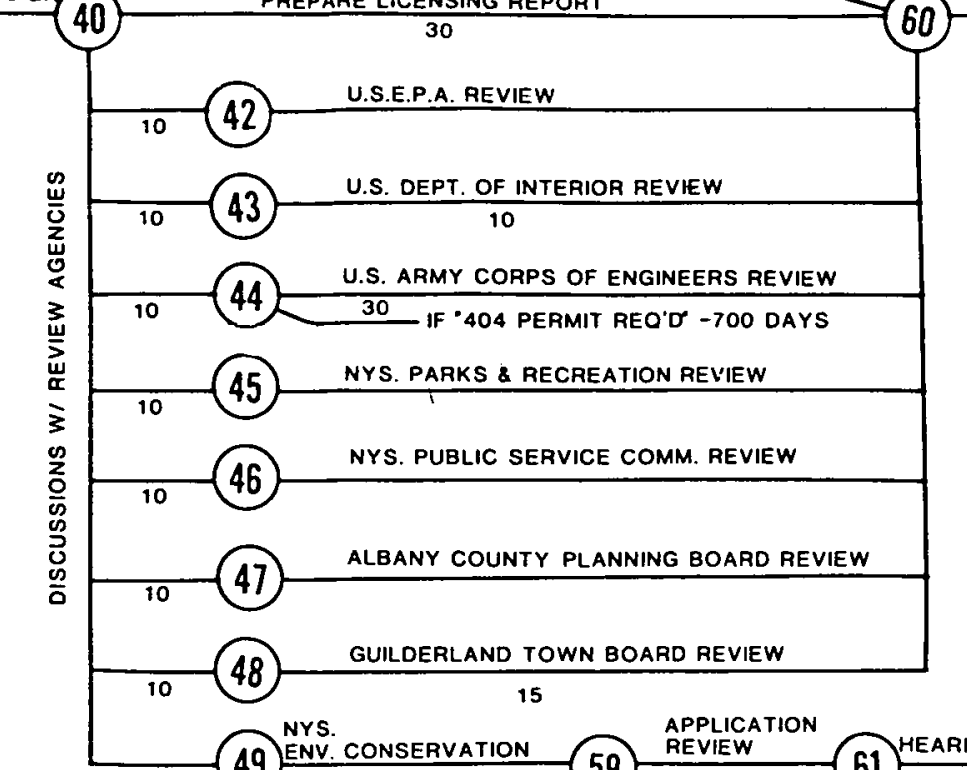

21)-(54)

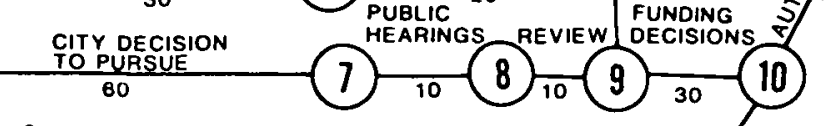

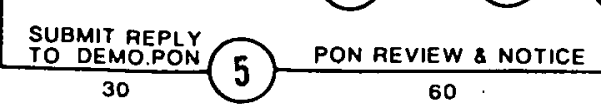

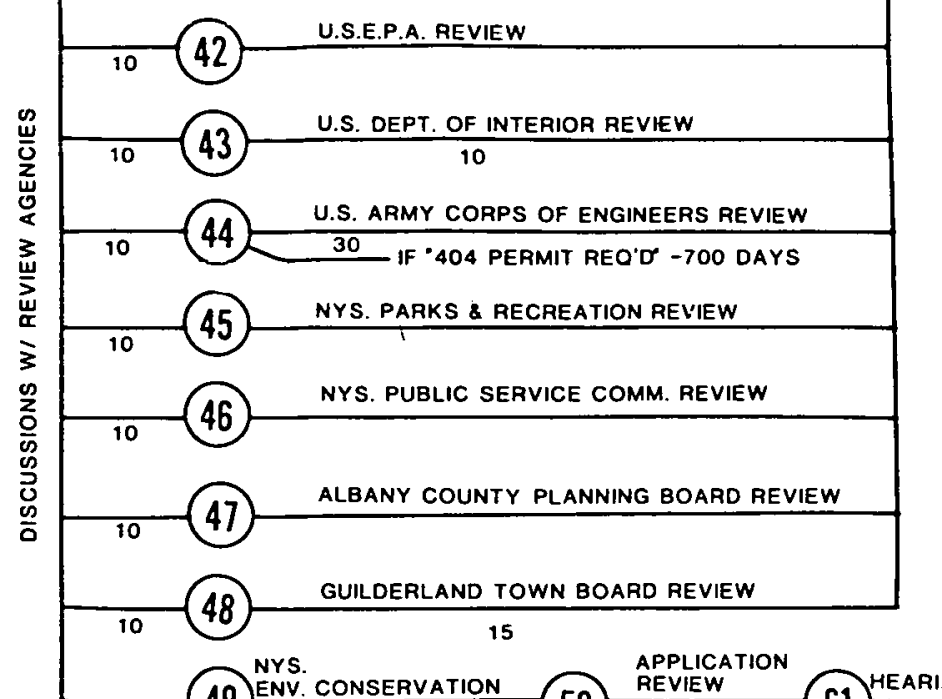

10 (49) $\frac{\text { NYS . CONSERVATION }}{15}$ (59)

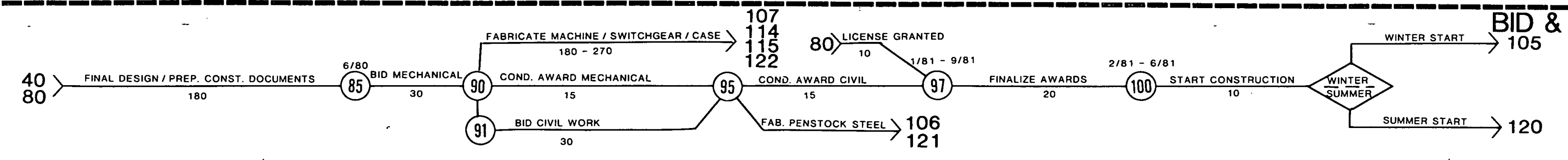

2/81 1 BRACE/DEWATER INTAKE, FORM, POUR FINISH PLACE, FORM, POUR, OUTFITEQUUP. SWITCHGEAR

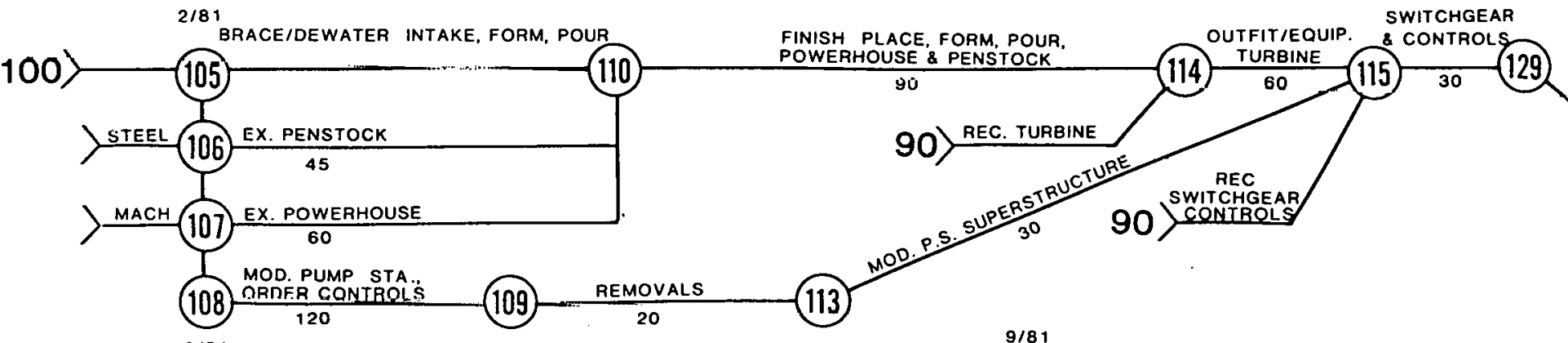

6/81 BRACE DEWATFR INTAKE. EQRM, POUR

100

$>$ STEEL (121) EXC. PENSTOCK, PLACE, B'FLL

$>$ MACHE

$\frac{25,45,20}{123} \frac{\text { MOD. PUMP STATION. ORDER CONTROLS }}{120}$

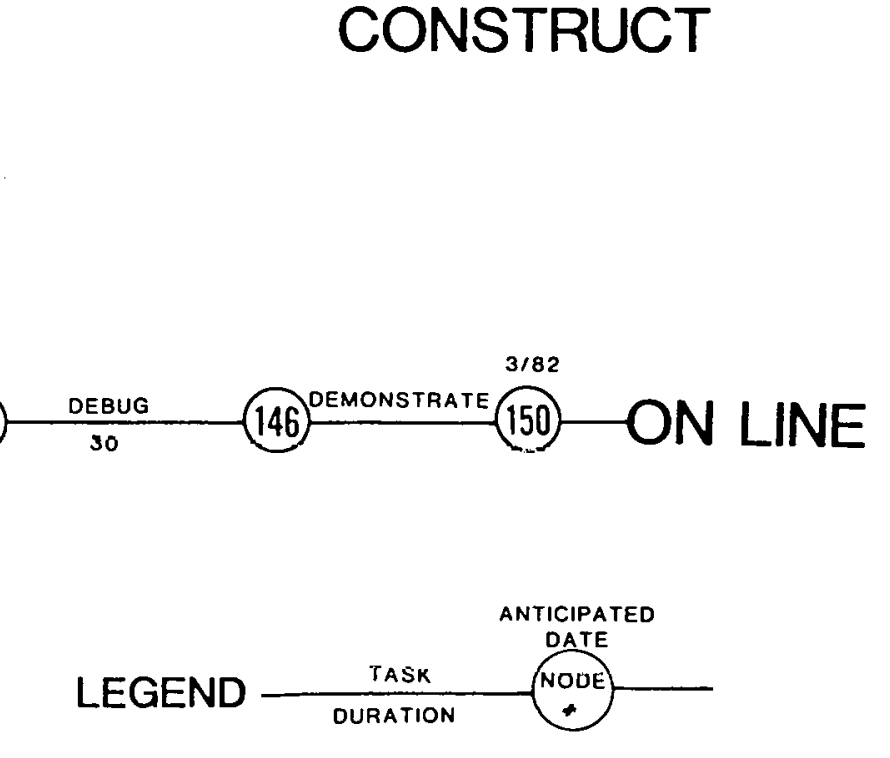




\section{SYNOPSIS AND RECOMMENDATIONS}

Recommended Project

The recommended project for implementation is project $B$. This project is considered to maximize potential hydroelectric generating capacity at the existing Normans Kill site. This project would produce $68 \%$ of potential hydroelectric capacity at the site. In this project, a new intake would be constructed at Bay 5 of the existing reinforced concrete dam. The intake (Figures 71, 71A) would consist of a reinforced concrete appendage to the upstream face of the dam with the outer walls of the intake resting upon buttresses 5 and 6 . The intake platform would be situated at Elevation 264, above design flood high water. Access to the intake platform would be via an internal stairway within the intake and the existing catwalk and stairway at the south abutment of the dam. The intake would consist of a stop log recess with a coarse grating consisting of reinforced concrete columns approximately two feet on center. Following the stop log recess, an intake sluice approximately eight feet square will be situated to control flow to the bar rack and penstock. A bar rack approximately 100 square feet in area will consist of $A 242$ steel bars approximately two inches on center with a net to gross area ratio of 0.65 . Velocity through the bar rack will be limited to two feet per second to minimize problems of frazil ice. The bar rack will be manually cleanable from the intake platform with provision made for a trash sluiceway at Elevation 257. Following the intake rack, a concrete transition to the welded steel penstock will be made exiting the dam with an invert elevation of approximately 21.2. Due to the minimal existing spillway capacity during standard project design flood and because of the fact that the proposed intake would block or eliminate approximately 18 feet of weir crest, an overflow siphon will be constructed integral with the intake on either side of it. The siphons will have a flow capacity equal to the capacity of the existing 18 foot section of crest. The sluice gate will be motor operated controllable from the powerhouse. Necessary railings and personnel safeguards will also be provided. 
The penstock under Project B consists of a 72 inch welded steel conduit encased in concrete below the existing streambed. After embedment of the penstock beneath the streambed, the existing streambed rock would be replaced to its original state presenting a aesthetic appearance similar to that now existing. It is anticipated that construction of this penstock would involve blasting and removal of the streambed rock during low or no-flow periods during the construction season.

The powerhouse under Project $B$ will consist of an underground vault constructed beneath the streambed (Figures $72,73,73 \mathrm{~A}$ ). The powerhouse will be constructed of reinforced concrete and will house a horiziontal axial flow tubular type turbine. The powerhouse will connect to an entry/access riser located adjacent to French's Mill Road. The connection to this access structure will be through an eight foot square reinforced concrete tunnel. Certain operating controls and local control panels for the turbine/generator will be located in this access structure. Switch gear associated with Project B (see Figure 75 ) will include generator protection relaying, an automatic synchronizing device, phase differential protective relays, overcurrent relaying, and overhead line protection relays. Switch gear from the generator to connection with the 2,400 volt bus will be located at the access structure above high water level. The switch gear will also include an above ground 2400 volt overhead transmission line to the existing pumn/rontrol station where the necessary motor starters, step-up transformers and utility tie will be located.

The axial flow tubular machine suggested for Project $B$ will operate at a maximum design flow of 200 cfs, a gross head of 64.4 feet, and a corresponding net head of 60.9 feet at design flow. The runner will have an approximate diameter of 35 inches and will be a five blade type. Setting of the turbine will be approximately four feet below design tail water. The turbine will operate with a specific speed of approximately 120 and will be connected through a speed increaser to a 1,000 KVA generator. The generator will be a three-phase synchronous and operated at 2,400 volts AC. The turbine 
will be equipped with an inlet butterfly valve upstream from the runner and the runner will be of the adjustable blade type. The composite efficiency of the machine will exceed 81.0\%. Ancillary equipment included in project B will consist of a turbine control panel. Necessary control information to and from the local control panel will be transmitted to the facility computer to be located at the existing pumping/control station via a fiber optic digital telemetry line. Additional anciliary equipment located at the powerhouse will include a breathable gas fire extinguishing protection system and station battery (for operation under black-start conditions). A control. schematic indicating relationships of the various turbine control, safety and monitoring. devices and the relationships with the facility control system is shown on Figure 76. The controls necessary to operate the turbine will include voltage and amperage and power factor meters, watt hour meters, and ground flow controls. Sensing systems capable of sensing reservoir elevation as well as indications of stream flow, rainfall, and ground moisture from two locations in the watershed will serve as input to a $32 \mathrm{~K}$ core digital computer located at the pumping/control station. This computer will be programmed to operate the hydroelectric and pumping station facility in an unattended mode with minimal supervision from a host computer to be located at the Watervliet water Filtration Plant in the city of watervliet. Costs for this host supervisory computer are not included in the hydroelectric project. The on-site computer would include off-line disc storage, cathode ray tube input/output as well as a priority interrupt control structure. The computer will interface through communications modums to the remote watershed sensors as well as to the host computer.

The recommended mode of operation for the composed Project $B$ Normanskill Hydroelectric Facility will be fully automatic. Two automatic options will be allowed. The first automatic control option will permit the local turbine/generator control panel to automatically vary turbine load based upon reservoir level with a preset control deadband. The second automatic control option will 
permit the local turbine/generator control panel to automatically vary load in proportion to a load set point signal determined by a load decision routine operating in the on-site digital computer. This load decision routine serves as the keystone element in a control algorithm for the Normanskill Hydroelectric facility. It has been determined in the hydrologic phase of this feasibility assessment that optimum operation of the hydroelectric facility (that is, operation maximizing hydroelectric output in relation to the market benefit function while at the same time minimizing disruptions of reservoir level) will operate most efficiently if short-term predictions of reservoir inflows can be obtained. This relationship is due in part to the large size of the watershed in relation to the rather limited reservoir capacity. Anticipation of inflow conditions and moderate drawdown in anticipation of inflow will serve to capture runoffs from storm events which would otherwise be lost. While at certain times of the year it might be feasible to operate the hydroelectric generation equipment in an automatic mode with the reservoir level as the sole criteria for load decision, it is expected that for a large portion of the year this mode of operation will, be inadequate. It is anticipated that the reservoir level deadband necessary to achieve stable turbine operation may itself be of a magnitude similar to the maximum allowable three feet drawdown. For this reason, it is anticipated that for most of the operating year an operating model based upon the particular hydrologic characteristics of the watershed will serve as the load decision input to the turbine control. This control model will regulate the generator load point in relation to a calculated reservoir inflow using the storage characteristics of the reservoir as buffer. Data relating to the expected energy consumption patterns of the City will be programed into the model as well as decision rules based upon the negotiated power accounting rules between the City and Niagara Mohawk Power Corporation. The control model will decide, for example, on the basis of City power consumption whether to ration the expected inflows by operating at low load points so as to extend operation of water pumping units 
under City produced power or to generate at full load to offset anticipated in-city power consumption. The routine will use as its source data for prediction of the streamflow into the reservoir, current data received from the remote sensing stations as well as long-term historical data. The long-term historical data base used to initialize the control model will be that of this feasibility assessment. The means used for correlating rainfall and ground moisture to streamflow will be a multivariate analysis technique relating the observed coefficients to measured-streamflow responses. However, as an aid in determining and updating the appropriate changes in system characteristics and parameters, a technique known as a Kalman filter will be used. [Ref. 52]. This control method estimates the state, in this case the streamflow, by updating the estimation of the state at the previous time frame, using a weighted component of all observed variables at the current state. This technique lends itself quite readily to prediction of short-term phenomena such as are observed.in the response of a small reservoir to a large watershed. The Kalman filter technique has the added advantage of improving its own accuracy through feedback, requiring minimal computational capacity.

Economic Criteria

The proposed project has a present worth net cost of $\$ 3,099,800$. The benefit cost ratio is 2.36. The corresponding internal rate of return is 16.248. Cash flows for Project B are depicted on Figure 58. Since the cash flows depicted use the convention of capital cost shown as a lump sum disbursement rather than amortized, a schedule of receipts and disbursements has been prepared and is shown on Figure 77. This schedule represents a cash flow summary for a 50-year period for project B. This schedule depicts the capital cost amortized over 25 years utilizing the expected City of watervilet municipal bonding rate of 6.38 . Furthermore, the amortization cash flow is calculated using the "50\% Rule" as mandated by the New York State Division of Audit \& Control for municipal bonding. 


\section{Further Criteria}

It is a matter of historical precedent that electric generation construction costs are sometimes examined on a basis of cost per kilowatt installed. We would suggest that for a project such as Project $B$, operating over a wide range of load factor, that this yardstick is not relevant. However, based upon a total capital cost of $\$ 1,747,000$ and with an installed net electric generating capacity of 842 kilowatts, the net cost per.installed kilowatt is $\$ 2,075$.

Similarly, a unit cost per kilowatt hour produced is sometimes used as a guideline. In this particular case, with a unique benefit cost structure, unique considerations, labor-saving, etc., it is not felt this unit pricing guideline is an accurate indication of project feasibility. However, the first year operating cost (which would be expected to be the highest operating cost due to the $50 \%$ amortization rule on capital cost) would be approximately $\$ 0.041$ per kilowatt hour. Other values for this unit price calculated for various years of the expected life of the facility are as follows:

\section{Year. $\quad$ Cost per Kilowatt Hour}

$\begin{array}{lr}\text { Year } 1 & \$ 0.041 \\ \text { Year } 10 & 0.023 \\ \text { Year } 20 & 0.014 \\ \text { Year } 30 & +0.103 \\ \text { Year } 40 & +0.228 \\ \text { Year 50 } & +0.484\end{array}$

It is estimated that the proposed hydroelectric generating facility at the French's Mills site, City of watervliet Reservoir will replace approximately 6,000 barrels of foreign oil per year. This is based upon the assumption that the marginal power produced by the local utility is foreign oil produced and assumes a 308 conversion efficiency with an oil energy equivalent of $5.8 \times 10^{6}$ BTU per barrel [Ref. 81]. 


\section{Conclusions}

Based upon technical, environmental and economic feasibility, the site owner, the City of Watervliet, New York, should pursue implementation of selected Project B for hydroelectric generation at the Watervilet Reservoir at French's Mills. 


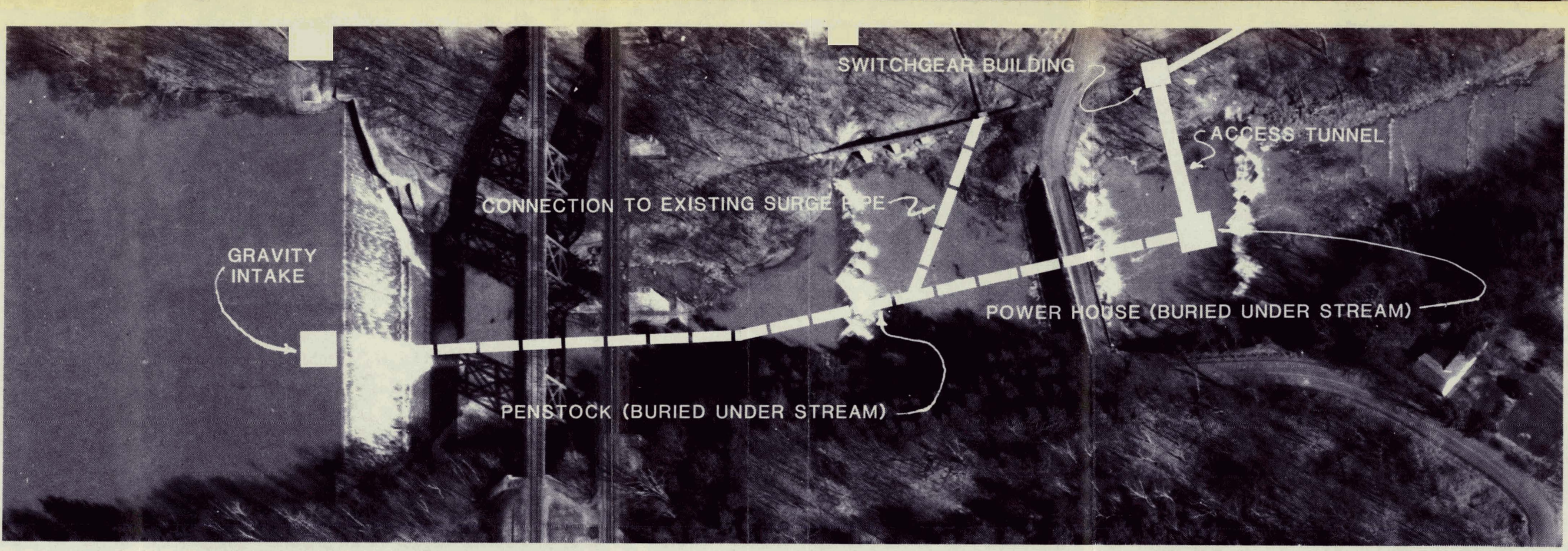

.
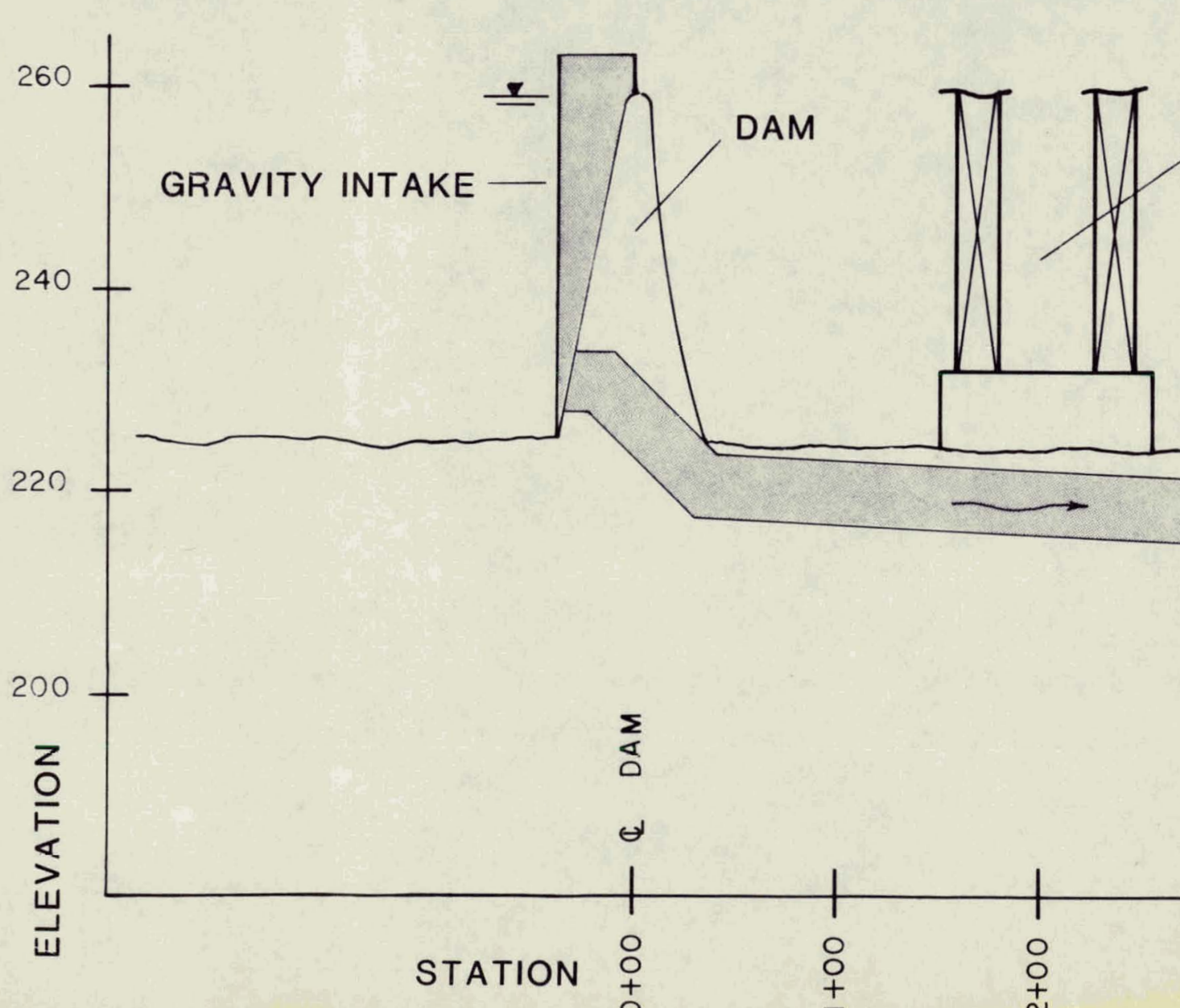

R.R. TRESTLE

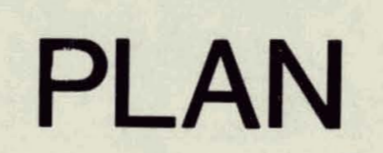

(T) TRESTE

PENSTOCK

(SEE FIGURE 41H)

NATURAL STREAMBED

HIGHWAY BRIDGE
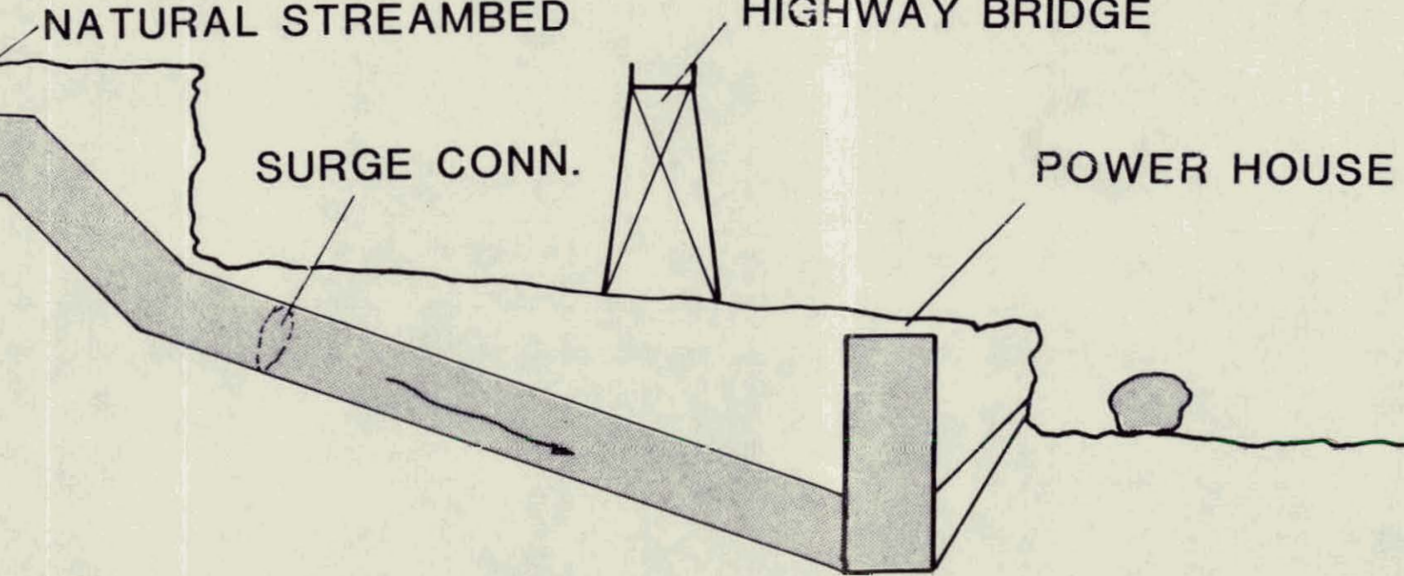


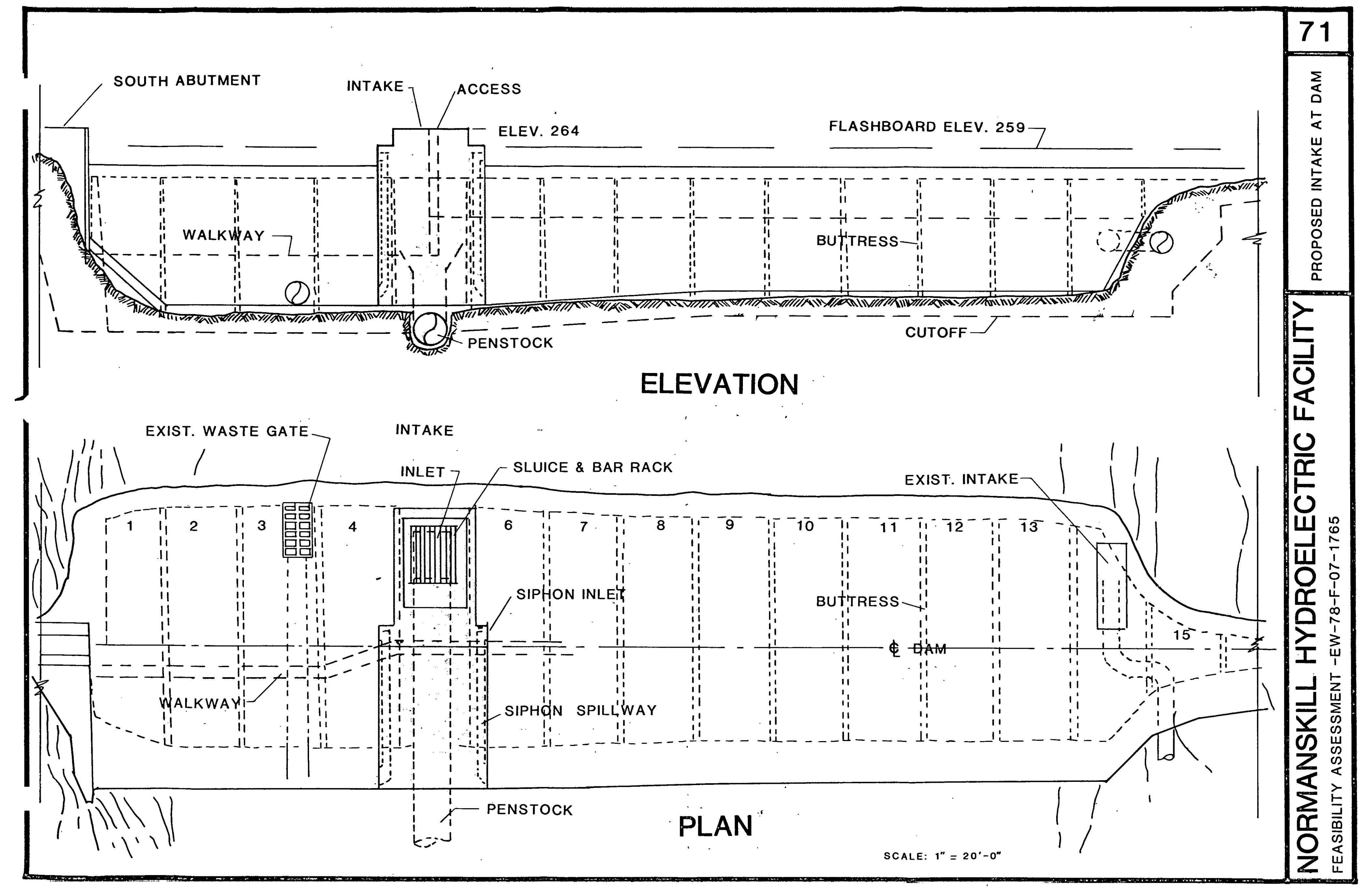




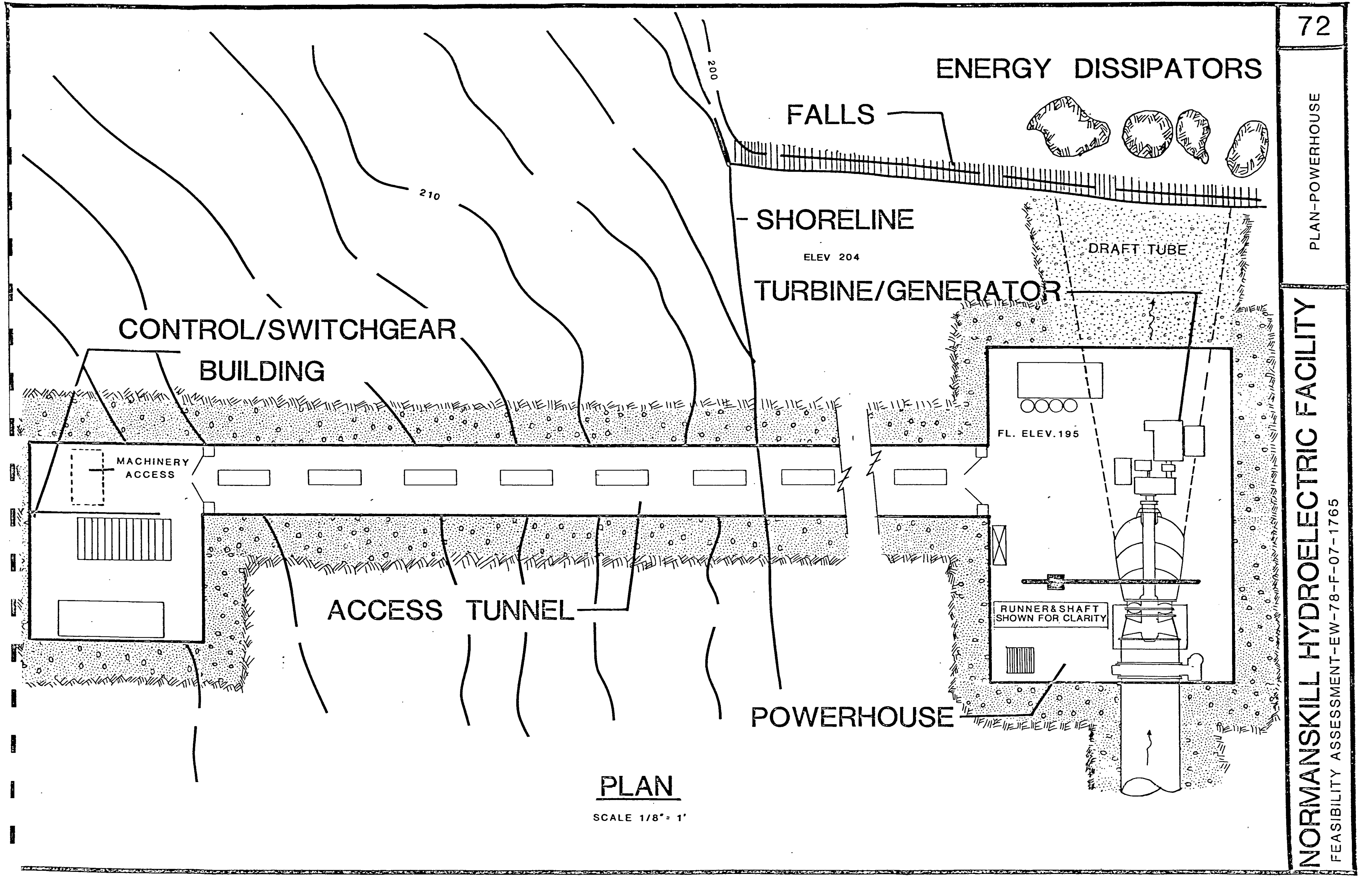




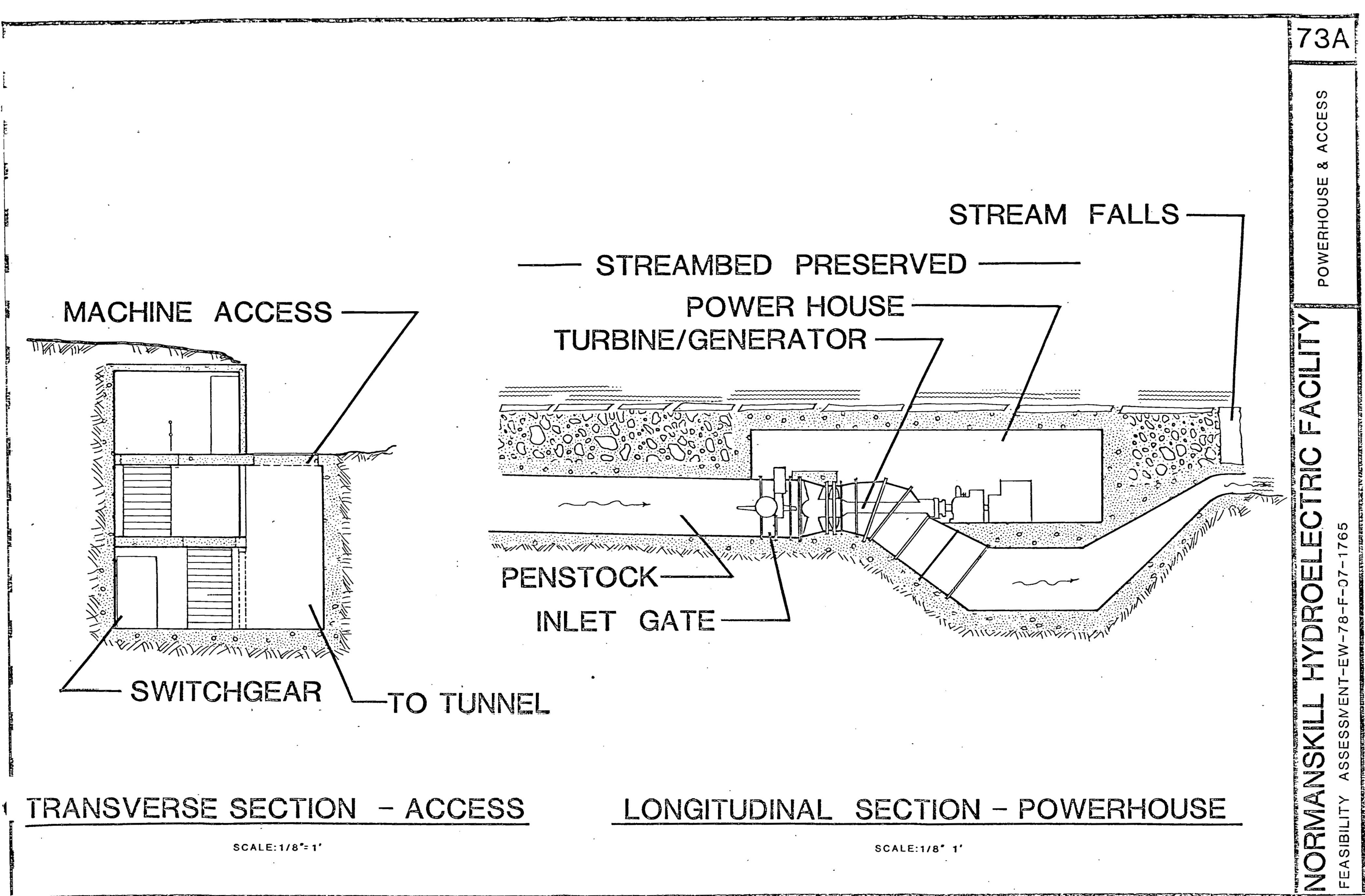




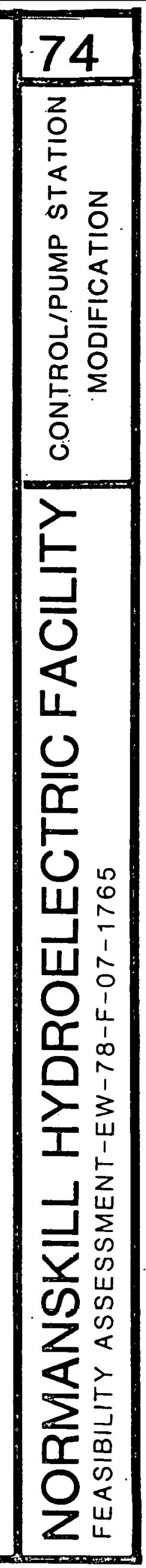




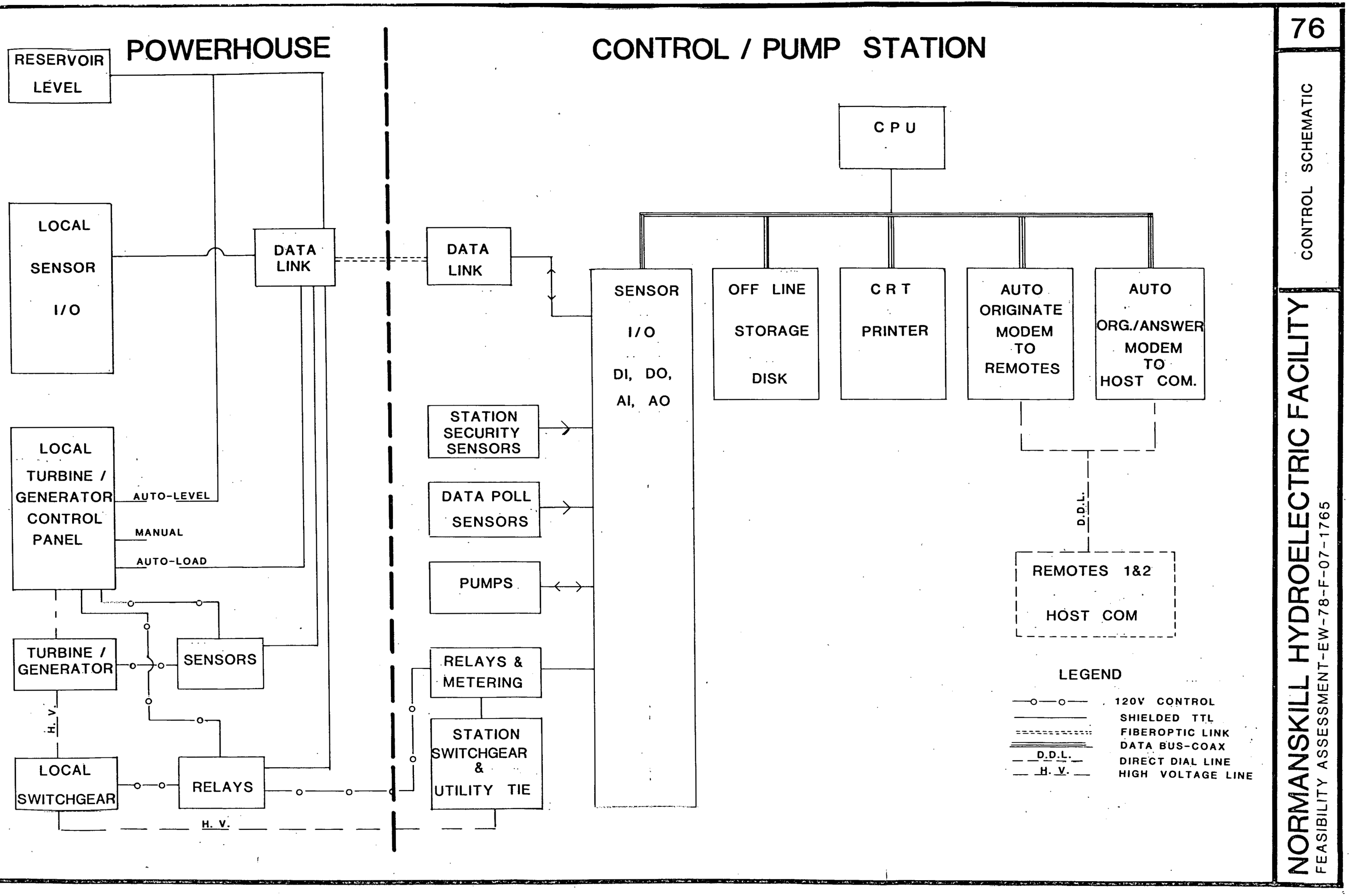


SCHEDULE OF CREDITS AND DISBURSEMENTS

PROJECT B

$\sum_{0}^{\infty}$

iv

II

$0 \%$

1.

음

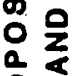

$\div<$

문

它

PROJECT 
NORMAIHS KILL HYTIRO-ELECTRIC FEASIEILITY STUIY

SCHEIIULE OF CREIITS ANII IIISEURSEMENTS (CFP3)

PROJECT E

50 YEAR ANALYSIS PERIOII ASSUMING 8.000 PERCENT [IISCOUHT RATE WITH 25 YEAR BONIIS 6.300 PERCENT

\begin{tabular}{|c|c|c|c|c|c|c|c|}
\hline YR. & CAP . REC. & $+\quad$ LAEIOR & PDWER & $+0 \& M$ & $+P W R \cdot C R$ & NET & CUM. $\mathrm{PW}$ \\
\hline-- & $-1---$ & --O- & & & & & \\
\hline 1 & -165984 & -19709 & -3745 & -57200 & 76826 & -169812 & -157233 \\
\hline 2 & -163626 & -20853 & -4007 & -59488 & 82204 & -165770 & -299354 \\
\hline 3 & -161196 & -22062 & -4288 & -61868 & 87958 & -161456 & -427523 \\
\hline 4 & -158691 & $-2334 ?$ & -4588 & -64342 & 94115 & -156848 & -542811 \\
\hline 5. & -156113 & -24696 & -4909 & -66916 & 100703 & -151931 & -646213 \\
\hline 6. & -153462 & -26128 & -5253 & -69593 & 107752 & -146684 & -738649 \\
\hline 7. & -150738 & -27643 & -5620 & -72376 & 115295 & -141082 & -820969 \\
\hline 8. & -147940 & -29247 & -6014 & -75271 & 123366 & -135106 & -893962 \\
\hline 9 & -145069 & -30943 & -6435 & -78282 & 132001 & -128728 & -958358 \\
\hline 10 & -142124 & -32738 & -6885 & -81413 & 141241 & -121919 & -1014831 \\
\hline & -139106 & -34636 & -7367 & -84670 & 151128 & -114651 & -1064002 \\
\hline 12 & -136015 & -36645 & -7883 & -88057 & 161707 & -106893 & -1106451 \\
\hline 13 & -132850 & -58771 & -8434 & -91579 & 173027 & -98607 & -1142709 \\
\hline 14 & -129612 & -41019 & -9025 & -95242 & 185139 & -89759 & -1173268 \\
\hline 15 & -126300 & -43398 & -9657 & -99052 & 198098 & -80309 & -1198585 \\
\hline 16. & -122916 & -45916 & -10333 & -103014 & 211965 & -70214 & -1219080 \\
\hline 17. & -119457 & -48579 & -11056 & -107135 & 226803 & -59424 & -1235140 \\
\hline 18. & -115926 & -51376 & -11830 & -111420 & 242679 & -47893 & -1247125 \\
\hline 19. & -112320 & $-51+377$ & -12658 & -115877 & 259667 & -35565 & -1255366 \\
\hline & -108642 & -57531 & -13544 & -120512 & 277843 & -22386 & -1260169 \\
\hline & -104890 & -60868 & -14492 & -125332 & 297292 & -8290 & -1261816 \\
\hline & -101 & -64398 & -15506 & -130346 & 318103 & 6788 & -1260567 \\
\hline & -97 & -68 & -16592 & -135559 & 340370 & 22920 & -1256664 \\
\hline & -93 & -72 & -17753 & -140982 & 364196 & 40182 & -1250327 \\
\hline & -891 & -76266 & -18996 & -146621 & 389690 & 58658 & -1241762 \\
\hline 2 & 0. & -80689 & -20326 & -152486 & 416968 & 163467 & -1219601 \\
\hline & 0 & -85369 & -21749 & -158585 & 446156 & 180453 & -1197070 \\
\hline & 0 & -90 & -23271 & -164929 & 477387 & 198866 & 4019 \\
\hline & 0 & -95560 & -24900 & -1.71526 & 510 & 218818 & 0534 \\
\hline & 0 & -101102 & -26643 & -178387 & 546560 & 240428 & 5641 \\
\hline & 0 & -106966 & -28508 & -185522 & 584819 & 263823 & 2365 \\
\hline & 0 & -113170 & -30503 & -192943 & 625 & 289140 & 7730 \\
\hline & 0 & -119734 & -32639 & -200661 & 669559 & 316525 & 2760 \\
\hline & 0 & -126678 & -34923 & 208687 & 716429 & 346141 & -1027476 \\
\hline 35 & 0 & -134026 & -37368 & -217035 & 766579 & 378150 & -1001900 \\
\hline & 0 & -141799 & -39984 & -225716 & 820239 & 412740 & -976052 \\
\hline & 0 & -150023 & -42783 & -234745 & 877656 & 450105 & -949953 \\
\hline & 0 & -158725 & -45777 & -244135 & 939092 & 490455 & -923620 \\
\hline & 0 & -167931 & -48982 & 253900 & 1004828 & 534015 & -897072 \\
\hline 40 & 0 & -177671 & -52411 & -264056 & 1075166 & 581028 & -870327 \\
\hline 41 & 0 & -187976 & -56079 & -274618 & 1150428 & 631755 & -843401 \\
\hline & 0 & -198878 & -60005 & -285603 & 1230958 & 686472 & -816310 \\
\hline Th & 0 & -210413 & -64205 & -277027 & 1317125 & 745480 & 9069 \\
\hline 44 & 0 & -222617 & -68700 & -308908 & 1409323 & 809098 & 1694 \\
\hline & 0 & -235 & -73509 & -321265 & 1507976 & 877673 & +198 \\
\hline & 0 & -249 & -78654 & -334115 & 1613534 & 951575 & 5596 \\
\hline$?$ & 0 & -263 & -84160 & -347480 & 1726482 & 1031199 & 3899 \\
\hline 48 & 0 & -278934 & -90051 & -361379 & 1847335 & 1116971 & 1121 \\
\hline 49. & 0 & -275112 & -96355 & -375834 & 1976649 & 1209348 & 3274 \\
\hline 50. & $\quad 0$ & -312229 & -103100 & -390868 & 2115014 & 1308817 & 5360 \\
\hline
\end{tabular}


Books

1. Anderson \& Settle. Benefit-Cost Analysis: A Practical Guide. Lexington, Mass.: Lexington Books, 1977.

2. Biswas (Ed.). Systems Approach to Water Management. New York: McGraw-Hill.

3. Chow. Handbook of Applied Hydrology. New York: McGrawHil1, 1964 .

4. Creager \& Justin. Hydro-electric Handbook. New York: John Wiley \& Sons. 1950 .

5. DeGarmo \& Canada. Engineering Economy. New York: MacMillan Company, 1973.

6. Fiering \& Jackson. Synthetic Streamflows. Washington, D. C.: American Geophysical Union Water Resources, 1971.

7. French. Historical and Statistical Gazetteer of New York State. 1860 .

8. Frost. How to Use Cost Benefit Analyses in Project Appraisal. New York: John Wiley \& Sons, 1975.

9. Gibson. Hydroelectric Engineering-Vol. I. Glasgow, Scotland: Bladee \& Son, Ltd., 1921.

10. Gibson. Hydroelectric Engineering-Vol. II. Glasgow, Scotland: Bladee \& Son, Ltd., 1922 .

11. Grant \& Ireson. Principles of Engineering Economy. New York: Ronald Press company, 1970.

12. Gregg. Old Hellebergh: Guilderland, New York: Guilderland Historical Society, 1975.

13. Hall \& Dracup. Water Resources Systems Engineering. New York: MCGraw-Hill Company, 1968.

14. Hino. Prediction of Flood and Streamflow by Modern Control and Stochastic Theories. Proceedings, Second International IAHR Symposium on stochastic. Hydraulics. Fort Collins, Colorado. Water Resources Publication. 1977.

15. Howe. Benefit-Cost Analysis to Water system Planning. washington, D.C.: American Geophysical Union, 1971. 
16. Howell and Tenney. History of the County of Albany. Munsell and Co.: New York, 1886.

17. Knetsch. Outdoor Recreation and Water Resources Planning. Washington, D.C.: American Geophysical Union, 1974.

18. LUNR. Land Use \& National Resource Inventory of New York State. Albany, New York: NYS Office of Planning Services, 1974.

19. Major. Multiobjective Water Resource Planning. Washington, D.C.: American Geophysical. Union, 1977.

20. Maas, Hutschmidt, Dortman, Thomas, Marglin, Fair. Design of Water Resource systems. Cambridge, Mass.: Harvard University Press, 1966.

21. Meier. Stochastic Population Dynamics for Regional Water Supply and Waste Management Decision Making. Amhurst, Mass.: University of Massachusetts.

22. Mikese11. The Rate of Discount for Evaluating Public Projects. Washingtón, D.C.: American Institute for Public Policy Research, 1977.

23. Muller. Hydroelectrical Engineering. New York: G. E. Stechert.\& Co.., 1921.

24. Park. Cost Engineering Analysis. New York: John Wiley \& Sons., 1973.

25. Parker. Landmarks of Albany County, New York. Mason and Company: Syracuse, New York, 1877.

26. Ritchie. Prehistoric Man in the Pine Bush. In Pine Bush, Don Rittner (Ed.), 1976.

27. Rose. Engineering Investment Decisions. Amsterdam, The Netherlands: Elsevier Scientific Publishing Company, 1970 .

28. Russel1, Arey \& Kates. Drought and Water Supply. Baltimore: Resources for the Future, Inc., 1970.

29. Salokongas (Ed.). Waterpower in Finland. Finland: Finnish Water Power Assn., 1968.

30. U.S: Department of Agriculture. Erosion and Sediment Inventory. Soil Conservation Service, 1975.

31. U.S. Department of the Interior, Bureau of Reclamation. Design of Small Dams. Washington, D.C.: U.S. Government Printing Office, 1974. 
32. Wegmann. The Design and Construction of Dams. New York: John wiley \& Sons, Inc., 1927.

33. Wiest \& Levy. A Management Guide to PERT/CPM. Englewood Cliffs, N.J.: Prentice Hall, Inc., 1972.

Periodicals

34. Armstrong. Investment Decision on Hydroelectric Development. Water Power, February 1971.

35. Askew. Optimum Reservoir Operating Policies and the Imposition of a Reliability Constraint. Water Resources Research, Vol. 10, No. I, February 1974.

36. Beard. Use of Interrelated Records to Simulate Streamflow. Journal of Hydraulics Div., A.S.C.E., september 1965.

37. Borland and Miller. Distribution of Sediment in Large Reservoirs. Journal of Hydraulics Div., American Society of Civil Engineers, 1958.

38. Burges. Use of Forecasted Seasonal Runoff Volumes in Reservoir Management. American Society of Civil Engineers. (Reprint 3387), 1979.

39. Carson, Samuelson. Low Head Turbines Draw Attention. Power, March 1978 .

40. Cassidy \& Shirra. Treatment of Inflation in Engineering Economic Analysis. I.E.C.E./A.S.M.E./A.S.C.E. Joint Power Generation Conference Paper F-76 6DI-5, 1976.

41. Chiu, Isu. Kalman Filter in Open Channel Flow Estimation. Journal of the Hydraulics Division, A.S.C.E. Vol. 104, No. H 8, August 1978.

42. Chow and Ramaseshan. Sequential Generation of Rainfall and Runoff Data. 'Journal of Hydraulics Div., A.S.C.E., July 1965.

13. Duckstein, Stidarovsky, Yakowitz. Bayes Design of a Reservoir under. Random Sediment Yield. Water Resources Research, Vol. 13, No. 4, August 1977.

44. Fiering. Queuing Theory and Simulation in Reservoir Design. Journal of Hydraulics Div., A.S.C.E., November 1961. 
45. Fiering. Multivariate Technique for Synthetic Hydrology. Journal of Hydraulics Div., A.S.C.E., September 1964.

46. Feingold. Energy Forecasting - Can Hindsight Improve Our Foresight? The Engineering Economist, Vol. 2I, No: l., American Society Engineering Education.

47. Fuller. Generation of Ungauged Streamflow Data. Journal of Hydraulics Div., A.S.C.E., March 1978.

48. Fults, Hancock, Logan. A Practical Monthly Optimum Operations Model. Journal of the Water Resources Planning \& Management Division, A.S.C.E., Vol. 102, No. WRI, April 1976.

49. Hall, Roe. Hydropower Project Output Optimization. Journal of Power Division, A.S.C.E.; Vol. 92, No. Pol; January 1966.

50. Hely. Areal Variations at Mean Annual Runoff. Journal of Hydraulics Div., A.S.C.E., September 1964.

51. Hanke, Carver, Bugg. Project Evaluation During Inflation. Water Resources Research. Vol. II, No. 4, August 1975.

52. Kalman. A New Approach to Linear Filtering and Prediction Problems. A.S.M.E. Journal of Basic Engineering, March 1960.

53. Koelyer and Lara. Densities and Compaction Ratio of Deposited Sediments. Journal of Hydraulics Div., American Society of Civil Engineers, April 1958.

54. Little. The Use of Storage Water in a Hydroelectric System, Journal of Operations Research. Vol. 3; 1955.

55. Lutz \& Cowles. Estimation Deviations. Their Effect upon the Benefit-cost Ratio. The Engineering Economist, Vol. 16, No. 1, American Society Engineer Education.

56. Maughan and Kowano. Project Yields by a Probability Method. Journal of Hydraulics Div., A.S.C.E., May 1963.

57. Menley. Calibration of Hydrological Model Using Optimization Techniques. Journal of the Hydraulics Division, American Society of Civil-Engineers. Vol. 104, No: HY2, February 1978. 
58. Ragade, Hipel, Unny: Metarationality in Benefit-Cost Analysis. Water Resources Research, Vol. 12, No. 5, October 1976.

59. Solow. What we know and Don't Know About Inflation. Technology Review, Vol. 81, No. 3. Cambridge, Mass.: Massachusetts Institute of Technology, January 1979.

60. Stall and Neill. Calculated Risks of Impounding Reservoir Yield. Journal of Hydraulics Div., A.S.C.E., January 1963.

61. Todini. Using a Desk-Top Computer for an On-Line Flood Warning System. IBM Journal of Research \& Development, Vol. 22, No. 5, september 1978.

62. Van Horne. Variation of Project Life As a Means for Adjusting for Risk. The Engineering Economist, Vol. 21, No. 3, American Society Engineering Education.

63. Williams and Berndt. Sediment Yield Computed with Universal Equation. Journal of the Hydraulics Div., American Society of Civil Engineers, December 1972.

\section{Reports}

64. Albany Common Counci1. Annual Report for 1891. January 1891.

65. Blakemore and Hartgen. Stage I and Stage II Archaeological Survey of the Lizzie \#2 Site. 1978.

66. Brinkman. Historical Data on the Town of Guilderland, New York. 19.45 .

67. Countryman. Nuisance Aquatic Plants in Lake Champlain. Lake Champlain Basin Study.

68. DeViona. Report to Albany water Supply Commission. 1891.

69. Funk. Recent Contributions to Hudson Valley Prehistory. New. York State Museum Memoir. 22. 1976.

70. Hering \& Buyert. Repurt to Albany water Supply Commission. 1891 .

71. Hill. Report on Albany Water Supply. New York, 1925.

72. McAlpine. Albany Water Supply Report. Albany, N. Y., 1850. 
73. N.Y. State Department of Health-Malcolm Pirnie, Engineers. Albany County Comprehensive Public Water supply study. White Plains, New York, May 1968.

74. Parker. The Archaeology of New York State. New York State Museum Bulletin 237-238, 1922.

75. Parsons, Brinkerhoff, Quade \& Douglas. Watervliet Water. Supply System Engineering Study. New York, March. 1964.

76. Salwen, Klein and Brumbach. Report on Archaeological Reconnaissance of Five Sections of the Guilderland Sewer System. Albany County, New York, 1975.

77. Solomon, Norcross \& Keis. Report on Watervliet Water Supply System. Troy, New York, January 1914.

78. Solomon, Norcross \& Keis. Water System Summary Report. March 04, 1914.

79. Shedd. Report to Albany Water Supply Commission. 1891.

80. Van der Veer. Report to Albany Water Supply Commission. 1891 .

\section{Government Documents}

81. Federal Energy Administration. Public Independence Final Task Force Report-Solar Energy. Washington, D.C., November 1974.

82. Federal Register-Vol. 38, No. 174. September 10, 1973 . Water Resource Council-Establishment of Principles and Standards for Planning.

83. Federal Register. Vol. 44, No. 46. 18 CFR Parts 4, 16, and 131. March 07, 1979.

84. Kalter, Gosse. Outdoor Recreation in New York State: Projections of Demand, Economic Value and Pricing Effects for the Period 1970-1985. Cornell University. Geneva, N. Y.: W. F. Humphrey Press, 1968.

85. McBride. Fisheries Survey of the Watervliet Reservoir. N.Y.S. Department of Environmental Conservation. Stamford, New York: 1978.

86. Blakemore and Hartgen. Stage I Cultural Resource Survey of the Upper Hungerkill Service Area, 1977. 
87. U. S. Office of Management \& Budget. Circular No. A-94, Discount Rates to be used in Evaluating Time-Distributed costs and Benefits., March 27, 1972.

$\underline{\text { Files }}$

88. Solomon, Norcross \& Keis. Letter to City of watervliet, 1914.

89. State of New York Conservation Commission. Water Supply Application 164 File. Filing date $05 / 25 / 1914$. 1582 File. Filing date $05 / 29 / 1943$.

\section{Correspondence and Communications}

90. Fenlon. New York State Department of Environmental Conservation. Personal communication, 1979.

91. Gibson. Dep. General Council, N.Y.S. Public Service Commission. Personal communication, 1979.

92. Gregg. Guilderland Town Historian, Town of Guilderland. Personal communication, 1979.

93. Johnson. Local expert on Albany County prehistory and history. Personal communication, 1979.

94. Leavitt. Albany Soil Conservation Service. U.S. Department of Agriculture, Albany, N.Y. Personal communication, 1978.

95. Iinday. Director, Office of Electric Power Regulation, Federal Energy Regulatory Commission. Correspondence, 1979.

96. Luckey. City of Watervliet Water Department. Personal communication, 1978 .

97. MCBride. N.Y.S. Department of Environmental Conservation. Stamford, N.Y. Personal communication, 1978.

98. .Mr. \& Mrs. Popisil. Guilderland Town residents: Personal communication, 1978 .

99. Stuzin. Director of Power, N.Y.S. Public Service Commission. Personal communication, 1979.

100. Vance. N.Y.S. Department of Environmental Conservation. Fish \& Wildlife Service, stamford, N. Y. Personal communication, 1979. 
101. Wood. New York State Department of Health, Environmental Health Center, Albany, N.Y. Personal communication, 1979.

Additional

102. U. S. Army Corps of Engineers. Phase I Inspection Report National Dam Safety Program - Watervliet Reservoir Dam. July 5, 1978.

103. U. S. Department of Agriculture, Albany County Soil Conservation, Service Soil Survey. 1942.

104. Smith. U. S. Department of Energy, Washington, D.C. Personal communications, 1979 .

105. Federal Register-Vol. 44, No. 69. Executive Order 12129. April 9, 1979. 